\title{
Cu-Catalyzed Oxidative Allylic C-H Arylation of Inexpensive Alkenes with (Hetero)Aryl Boronic Acids
}

\author{
Suman Pal, ${ }^{\mathrm{a}}$ Marine Cotard, ${ }^{\mathrm{a}}$ Baptiste Gérardin, ${ }^{\mathrm{a}}$ Christophe Hoarau, ${ }^{* \mathrm{a}}$ Cédric Schneider*a \\ ${ }^{a}$ Normandie Univ, UNIROUEN, INSA Rouen, CNRS, COBRA UMR 6014, 1 rue Tesnière 76821 Mont Saint \\ Aignan, Cedex, France \\ Email: cedric.schneider@univ-rouen.fr \& christophe.hoarau@insa-rouen.fr
}




\section{Table of contents}

Cu-Catalyzed Oxidative Allylic C-H Arylation of Inexpensive Alkenes with (Hetero)Aryl

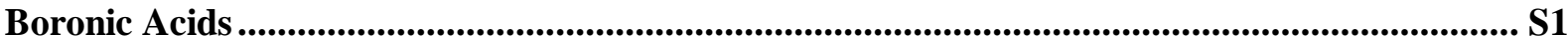

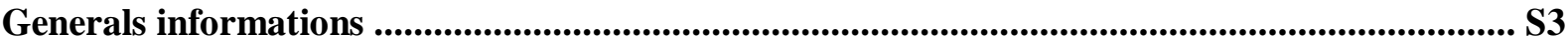

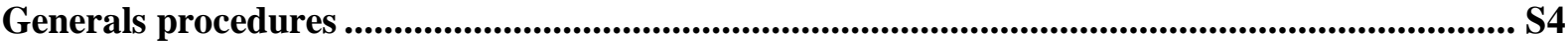

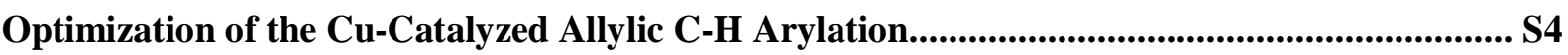

1. Table 1. Screening of Copper sources, Ligands and Oxidants............................................... 4

2. Table 2. Screening of Solvent, ratio of $\mathrm{Cu}_{2} \mathrm{O}$, Temperature and Reaction time........................S5

3. Table 3. Screening of Solvent, ratio of $\mathrm{Cu}_{2} \mathrm{O}$, Temperature and Reaction time........................S6

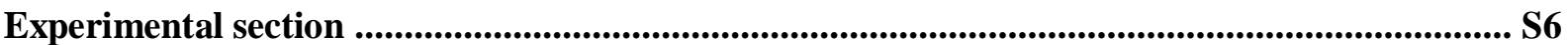

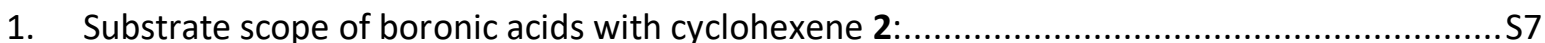

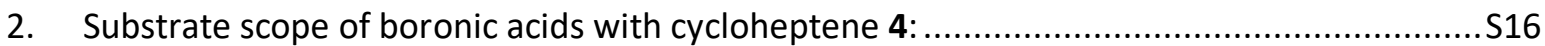

3. Substrate scope of boronic acids with cyclooctene 5 : .....................................................

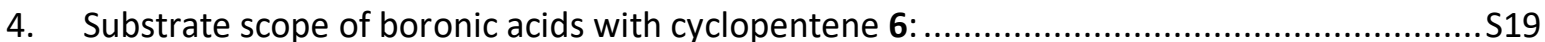

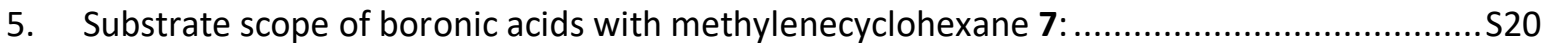

6. Substrate scope of boronic acids with allylbenzene derivatives 8-9: ...............................S21

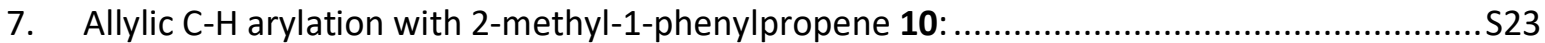

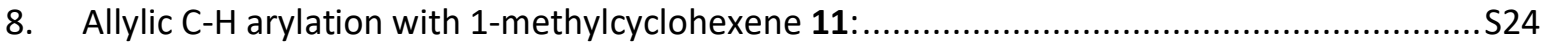

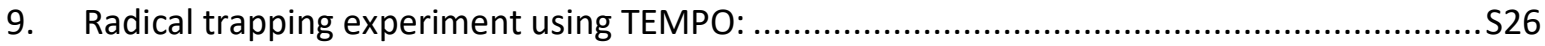

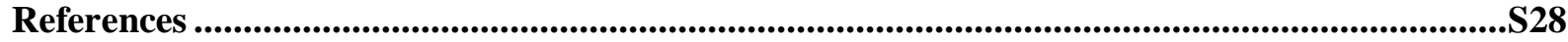

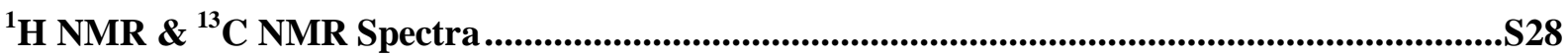

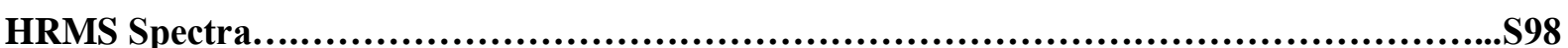




\section{Generals informations}

All boronic acids 1a-q, and alkenes 2 and 4-11 were purchased from commercial suppliers and used without further purification. All reactions were carried out under an argon atmosphere unless otherwise stated. Melting points were determined on a Fisher Scientific hot stage melting point apparatus and are uncorrected. ${ }^{1} \mathrm{H}$ and ${ }^{13} \mathrm{C}$ NMR spectra were recorded using a Bruker Avance-300 spectrometer operating at $300 \mathrm{MHz}{ }^{1} \mathrm{H}$ frequency, corresponding ${ }^{13} \mathrm{C}$ and ${ }^{19} \mathrm{~F}$ frequencies are 75 and $282 \mathrm{MHz}$ ). The chemical shifts are calibrated to residual proton and carbon resonance of $\mathrm{CDCl}_{3}\left({ }^{1} \mathrm{H} 7.26\right.$ and $\left.{ }^{13} \mathrm{C} 77.1 \mathrm{ppm}\right)$. In the ${ }^{13} \mathrm{C}$ NMR spectra, signals corresponding to $\mathrm{CH}, \mathrm{CH}_{2}$, or $\mathrm{CH}_{3}$ groups are assigned from DEPT. Chemical shifts $(\delta)$ are given in parts per million from tetramethylsilane (TMS) as internal standard. The following abbreviations are used for the proton spectra multiplicities: s: singlet, d: doublet, t: triplet, q: quartet, m: multiplet, bs: broad singlet, dd: doublet of doublets. Coupling constants $(J)$ are reported in Hertz $(\mathrm{Hz})$. IR spectra were recorded on Perkin Elmer Spectrum 100 FT IR spectrometers. HRMS were recorder on a LC Waters Acquity coupled to a Waters LCT Premier XE (Q-TOF) instrument. All reactions were monitored by thin-layer chromatography with Merck silica gel 60 F254 pre-coated aluminium plates $(0.25 \mathrm{~mm})$. Compounds were visualized by UV irradiation and/or spraying with a solution of potassium permanganate, followed by charring at $150{ }^{\circ} \mathrm{C}$. Flash column chromatography was performed on silica gel 60 (230-400 mesh, 0.040-0.063 mm). 


\section{Generals procedures}

\section{Optimization of the Cu-Catalyzed Allylic C-H Arylation}

1. Table 1. Screening of Copper sources, Ligands and Oxidants

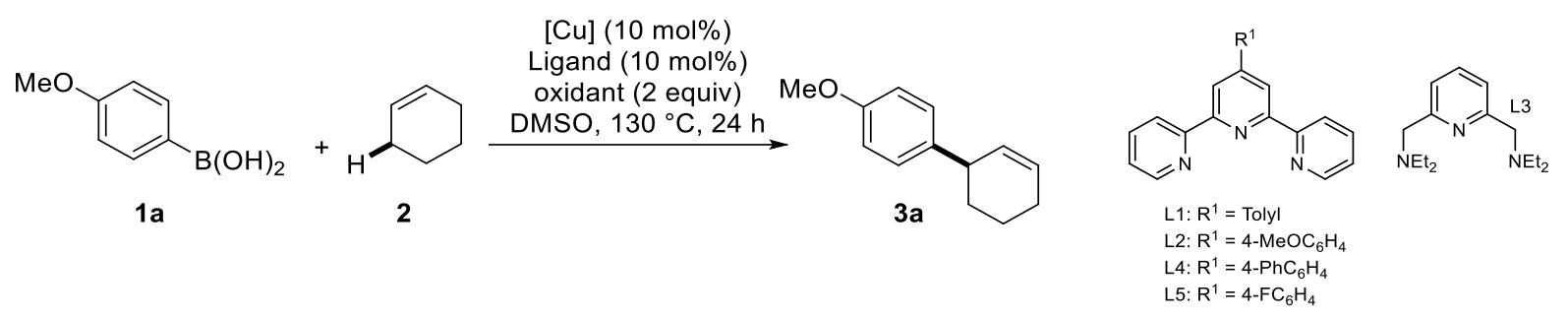

\begin{tabular}{|c|c|c|c|c|}
\hline Entry & [Cu] (10 mol\%) & Ligand & Oxidant & Yield (\%) ${ }^{\mathrm{a}}$ \\
\hline 1 & $\mathrm{Cu}(\mathrm{NCMe})_{4} \mathrm{PF}_{6}$ & L1 & DTBP & 53 \\
\hline 2 & CuTc & L1 & DTBP & 72 \\
\hline 3 & CuBrøDMS & L1 & DTBP & 45 \\
\hline 4 & CuOAc & L1 & DTBP & 42 \\
\hline 5 & IPrCuCl & & DTBP & 53 \\
\hline 6 & $\mathrm{Cu}(\mathrm{I}) \mathrm{SCN}$ & L1 & DTBP & 25 \\
\hline 7 & Cul & L1 & DTBP & 15 \\
\hline 8 & CuOTføtoluene & L1 & DTBP & 44 \\
\hline 9 & No $[\mathrm{Cu}]$ source & L1 & DTBP & n.r. \\
\hline 10 & $\mathrm{CuCl}$ & L1 & DTBP & 63 \\
\hline 11 & $\mathrm{Cu}_{2} \mathrm{O}$ & L1 & DTBP & $80(72)^{b}$ \\
\hline 12 & $\mathrm{Cu}_{2} \mathrm{O}$ & L1 & $\mathrm{BPO}$ & n.r. \\
\hline 13 & $\mathrm{Cu}_{2} \mathrm{O}$ & L1 & DCP & 24 \\
\hline 14 & $\mathrm{Cu}_{2} \mathrm{O}$ & L1 & TBHP & n.r. \\
\hline 15 & $\mathrm{Cu}_{2} \mathrm{O}$ & L1 & TBPB & n.r. \\
\hline 16 & $\mathrm{Cu}_{2} \mathrm{O}$ & L1 & NFSI & n.r. \\
\hline 17 & $\mathrm{Cu}_{2} \mathrm{O}$ & L1 & $\mathrm{K}_{2} \mathrm{~S}_{2} \mathrm{O}_{8}$ & n.r. \\
\hline 18 & $\mathrm{Cu}_{2} \mathrm{O}$ & L1 & DTBP & 72 \\
\hline 18 & $\mathrm{Cu}_{2} \mathrm{O}$ & L2 & DTBP & 75 \\
\hline 19 & $\mathrm{Cu}_{2} \mathrm{O}$ & L3 & DTBP & 32 \\
\hline 20 & $\mathrm{Cu}_{2} \mathrm{O}$ & L4 & DTBP & 16 \\
\hline 21 & $\mathrm{Cu}_{2} \mathrm{O}$ & L5 & DTBP & 21 \\
\hline 22 & $\mathrm{Cu}_{2} \mathrm{O}$ & Phen & DTBP & 7 \\
\hline 23 & $\mathrm{Cu}_{2} \mathrm{O}$ & BathoPhen & DTBP & 18 \\
\hline 24 & $\mathrm{Cu}_{2} \mathrm{O}$ & Phen-5,6-dione & DTBP & 14 \\
\hline 25 & $\mathrm{Cu}_{2} \mathrm{O}$ & 2,2'-Bipyridine & DTBP & 27 \\
\hline 26 & $\mathrm{Cu}_{2} \mathrm{O}$ & TMEDA & DTBP & 13 \\
\hline 27 & $\mathrm{Cu}_{2} \mathrm{O}$ & BOX & DTBP & 26 \\
\hline 28 & $\mathrm{Cu}_{2} \mathrm{O}$ & Xantphos & DTBP & 47 \\
\hline 29 & $\mathrm{Cu}_{2} \mathrm{O}$ & dppf & DTBP & 40 \\
\hline 30 & $\mathrm{Cu}_{2} \mathrm{O}$ & $\mathrm{dppBz}$ & DTBP & 31 \\
\hline 31 & $\mathrm{Cu}_{2} \mathrm{O}$ & dppe & DTBP & 19 \\
\hline 32 & $\mathrm{Cu}_{2} \mathrm{O}$ & No ligand & & $25(12)^{b}$ \\
\hline
\end{tabular}

${ }^{a}$ Reaction conditions: 1a (1 equiv), 2 (10 equiv) [Cu] (10 mol\%), ligand (10 mol\%), Oxidant (2 equiv), DMSO, $130{ }^{\circ} \mathrm{C}, 24 \mathrm{~h}$. Yields are determined by crude ${ }^{1} \mathrm{H}$ NMR using dibenzylether as the internal standard. 'Isolated yields are in bracket. ${ }^{c_{3}}$ equiv of DTBP ware used. CUTC: Copper(I) thiophene-2- 
carboxylate (I). CuSCN: Copper thiocyanate (I). IPrCuCl: Chloro[1,3-bis(2,6-diisopropylphenyl)imidazol2-ylidene]copper(I). Phen-5,6-Dione: 1,10-Phenanthroline-5,6-dione. BathoPhen: Bathophenanthroline. dppBz: 1,2-Bis(diphenylphosphino)benzene. dppe: 1,2Bis(diphenylphosphino)ethane. BOX: 2,2'-Isopropylidenebis[(4S)-4-tert-butyl-2-oxazoline. DCP: Dicumyl peroxide. TBHP: tert-Butylhydroperoxide. TBPB: tert-Butyl peroxybenzoate. BPO: Benzoyl peroxide. DTBP: Di-tert-butyl peroxide.

2. Table 2. Screening of Solvent, ratio of $\mathrm{Cu}_{2} \mathrm{O}$, Temperature and Reaction time

\begin{tabular}{|c|c|c|c|c|}
\hline & & $\begin{array}{l}X X \text { mol\%) } \\
\text { D mol\%) } \\
(2 \text { equiv) } \\
\text { t ( }(0.2 \mathrm{M}) \\
\text { c), } t(\mathrm{~h})\end{array}$ & & \\
\hline $\mathrm{Cu}_{2} \mathrm{O}$ (XX mol\%) & Solvant & $\mathrm{T}\left({ }^{\circ} \mathrm{C}\right)$ & $t(h)$ & Yield (\%) \\
\hline 10 & DMSO & 130 & 24 & $80(72)^{b}$ \\
\hline 10 & DMF & 130 & 24 & n.r. \\
\hline 10 & DMAC & 130 & 24 & n.r. \\
\hline 10 & DCE & 130 & 24 & 34 \\
\hline 10 & Chlorobenzene & 130 & 24 & n.r. \\
\hline 10 & THF & 130 & 24 & n.r. \\
\hline 10 & Sulpholane & 130 & 24 & $75(55)^{b}$ \\
\hline 10 & $\begin{array}{c}\text { Neat (0.3 M } \\
\text { Cyclohexene) }\end{array}$ & 130 & 24 & n.r. \\
\hline 5 & DMSO & 130 & 24 & 54 \\
\hline 20 & DMSO & 130 & 24 & 75 \\
\hline 10 & DMSO (0.3 M) & 130 & 24 & 60 \\
\hline 10 & DMSO (0.1 M) & 130 & 24 & 70 \\
\hline 10 & DMSO (0.05 M) & 130 & 24 & 53 \\
\hline 10 & DMSO & 110 & 24 & 70 \\
\hline 10 & DMSO & 80 & 24 & 52 \\
\hline 10 & DMSO & 60 & 24 & 17 \\
\hline 10 & DMSO & 80 & 48 & $92(83)^{b}$ \\
\hline
\end{tabular}

${ }^{a}$ Reaction conditions: 1 a (1 equiv), 2 (10 equiv) $\mathrm{Cu}_{2} \mathrm{O}$ (XX mol\%), L1 (10 mol\%), DTBP (2 equiv), Solvent $(0.2 \mathrm{M}), \mathrm{T}\left({ }^{\circ} \mathrm{C}\right), \mathrm{t}(\mathrm{h})$. Yields are determined by crude ${ }^{1} \mathrm{H}$ NMR using dibenzylether as the internal standard. ${ }^{b}$ Isolated yields are in bracket. 
3. Table 3. Screening of Solvent, ratio of $\mathrm{Cu}_{2} \mathrm{O}$, Temperature and Reaction time

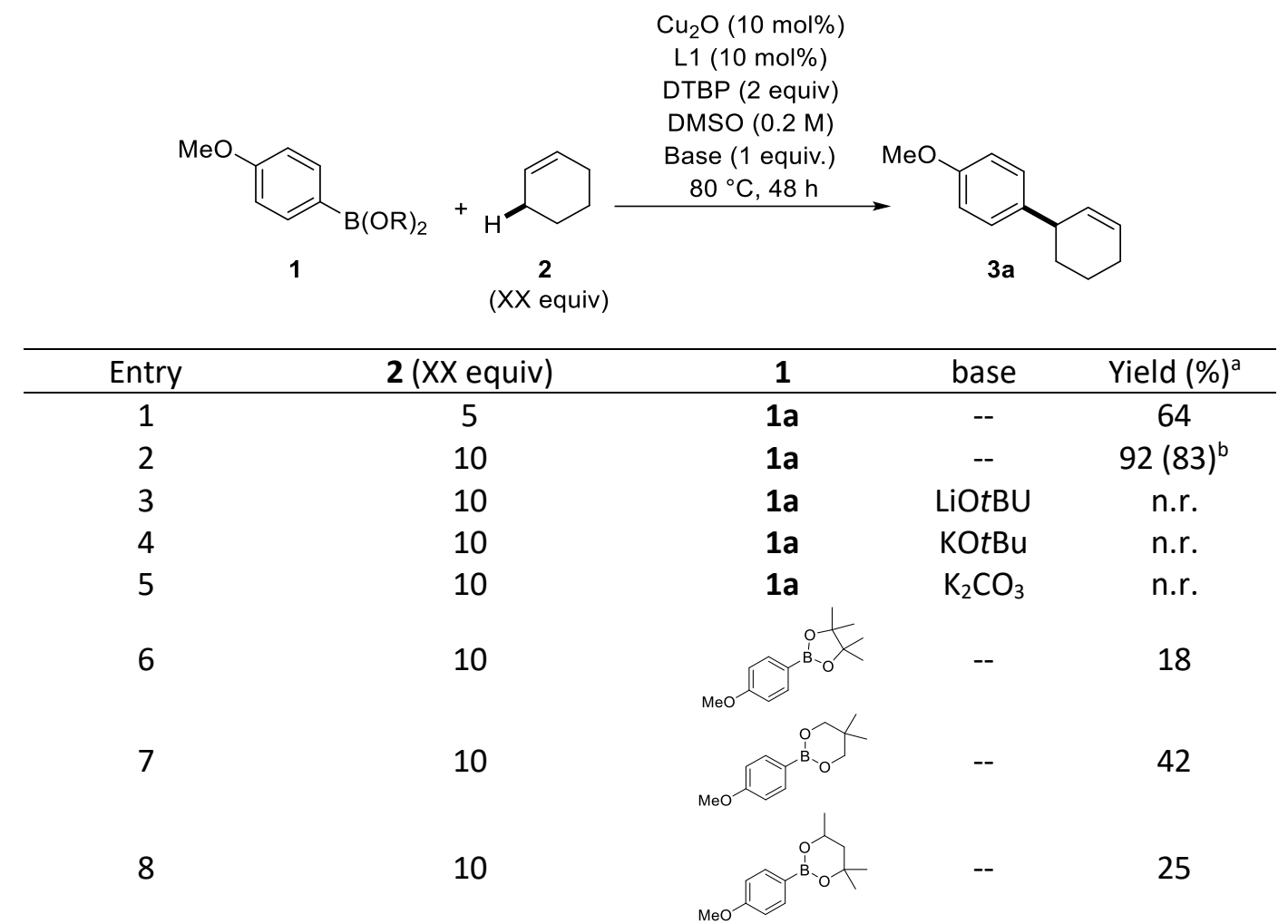

${ }^{a}$ Reaction conditions: 1 (1 equiv), 2 (XX equiv) $\mathrm{Cu}_{2} \mathrm{O}$ (10 mol\%), L1 (10 mol\%), DTBP (2 equiv), Base (1 equiv.), DMSO $(0.2 \mathrm{M}), 80^{\circ} \mathrm{C}, 48 \mathrm{~h}$. Yields are determined by crude ${ }^{1} \mathrm{H}$ NMR using dibenzylether as the internal standard. ${ }^{b}$ Isolated yields are in bracket.

\section{Experimental section}

\section{General procedure for allylic C-H arylation:}

Scheme 1: Cu-catalyzed oxidative Allylic C-H arylation of alkenes with boronic acids.

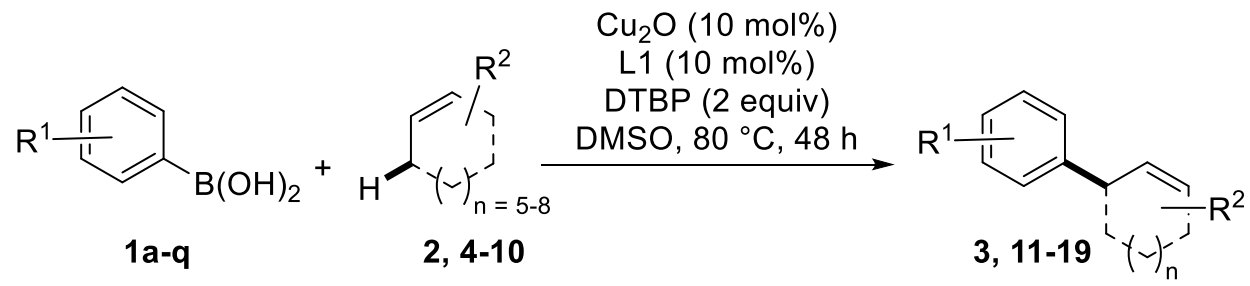

Boronic acid 1a-q (0.5 mmol, 1 equiv.), $\mathrm{Cu}_{2} \mathrm{O}(0.05 \mathrm{mmol}, 10 \mathrm{~mol} \%)$ and $\mathrm{L} 1$ (0.05 mmol, 10 mol\%) were placed in a sealed tube which was then evacuated and backfilled three times with nitrogen. DMSO (1.5 mL), alkene 2, 4-10 (5 mmol, 10 equiv.) and DTBP (1 mmol, 2 equiv.) were added and the mixture was allowed to heat at $80{ }^{\circ} \mathrm{C}$ using oil bath for $48 \mathrm{~h}$. The progress of the reaction was monitored by TLC. Upon completion the reaction mixture was filtered through celite and mixture was extracted twice with DCM. The combined organic phases were 
dried with $\mathrm{MgSO}_{4}$ and evaporated under reduced pressure and the crude product was purified by silica gel chromatography to yield the desired product in the indicated yield.

\section{Substrate scope of boronic acids with cyclohexene 2:}<smiles>COc1ccc(C2C=CCCC2)cc1</smiles>

3a

3-(4-Methoxyphenyl)cyclohexene (3a): The compound 3a was prepared according to the general procedure. Purification by flash chromatography in PE/EA (99:1, v/v) afforded 3a as a clear oil (78 mg, 83\% yield). Exhibited spectra data identical to previous reports. ${ }^{1}$ The reaction was scaled up from $0.5 \mathrm{mmol}$ to $5.0 \mathrm{mmol}$, and after purification by flash chromatography, the desired product 3a was obtained in $80 \%$ yield $(4.15 \mathrm{mmol}, 781 \mathrm{mg})$ IR (ATR) $v_{\max }$ : 2927, 2855, 2834, 1610, 1509, 1443, 1301, 1242, 1107, 1037, 882, $826 \mathrm{~cm}^{-1}$. ${ }^{1} \mathrm{H}$ NMR $\left(300 \mathrm{MHz}, \mathrm{CDCl}_{3}\right): \delta$ 7.18-7.13 (m, 2H), 6.88-6.83 (m, 2H), $5.88(\mathrm{ddd}, J=9.7,5.8$, $3.5 \mathrm{~Hz}, 1 \mathrm{H}), 5.74-5.67(\mathrm{~m}, 1 \mathrm{H}), 3.80$ (s, 3H), 3.41-3.34 (m, 1H), 2.13-1.95 (m, 3H), 1.80-1.48 $(\mathrm{m}, 3 \mathrm{H})$.

${ }^{13} \mathrm{C}$ NMR $\left(75 \mathrm{MHz}, \mathrm{CDCl}_{3}\right): \delta 157.9(\mathrm{C}), 138.8(\mathrm{C}), 130.5(\mathrm{CH}), 128.6(2 \times \mathrm{CH}), 128.2(\mathrm{CH})$, $113.7(2 \times C H), 55.3\left(\mathrm{CH}_{3}\right), 40.9(\mathrm{CH}), 32.7\left(\mathrm{CH}_{2}\right), 25.0\left(\mathrm{CH}_{2}\right), 21.1\left(\mathrm{CH}_{2}\right)$.

HRMS (EI ${ }^{+}$m/z: [M] ${ }^{+}$Calcd for $\mathrm{C}_{13} \mathrm{H}_{16} \mathrm{O}$ 188.1201; Found 188.1206.<smiles>Cc1ccc(C2C=CCCC2)cc1</smiles>

$3 \mathbf{b}$

3-(4-Methylphenyl)cyclohexene (3b): The compound 3b was prepared according to the general procedure. Purification by flash chromatography in Petroleum ether afforded $\mathbf{3 b}$ as a clear oil (65 mg, 76\% yield). Exhibited spectra data identical to previous reports. ${ }^{2}$ 
IR (ATR) $v_{\max }: 2925,2857,1721,1651,1604,1513,1449,1409,1349,1261,1182,1042,878$, $809 \mathrm{~cm}^{-1}$.

${ }^{1} \mathrm{H}$ NMR (300 MHz, $\left.\mathrm{CDCl}_{3}\right): \delta 7.15(\mathrm{~s}, 4 \mathrm{H}), 5.91(\mathrm{dt}, J=5.9,3.6 \mathrm{~Hz}, 1 \mathrm{H}), 5.74(\mathrm{dd}, J=10.1$, $2.1 \mathrm{~Hz}, 1 \mathrm{H}), 3.45-3.37$ (m, 1H), 2.36 (s, 3H), 2.14-2.00 (m, 3H), 1.81-1.53 (m, 3H).

${ }^{13} \mathrm{C} \mathrm{NMR}\left(75 \mathrm{MHz}, \mathrm{CDCl}_{3}\right): \delta 143.6(\mathrm{C}), 135.6(\mathrm{C}), 130.5(\mathrm{CH}), 129.0(2 \mathrm{xCH}), 128.2(\mathrm{CH})$, $127.7(2 \times C H), 41.5(\mathrm{CH}), 32.6\left(\mathrm{CH}_{2}\right), 25.1\left(\mathrm{CH}_{2}\right), 21.3\left(\mathrm{CH}_{2}\right), 21.0\left(\mathrm{CH}_{3}\right)$.

$\operatorname{HRMS}\left(\mathrm{CI}^{+}\right) \mathrm{m} / \mathrm{z}$ : $[\mathrm{M}-\mathrm{H}]^{+}$Calcd for $\mathrm{C}_{13} \mathrm{H}_{15}$ 171.1173; Found 171.1166.

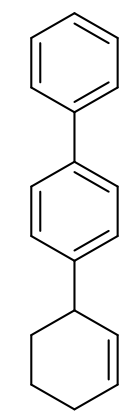

3c

3-(1,1'-Biphényl)cyclohexene (3c): The compound was prepared according to the general procedure. Purification by flash chromatography in Petroleum ether afforded $\mathbf{3 c}$ as a clear oil (84 mg, $72 \%$ yield). Exhibited spectra data identical to previous reports. ${ }^{3}$

IR (ATR) $v_{\max }: 3027,2929,2858,1721,1645,1600,1486,1447,1406,1262,1190,1075,832$, $762,695 \mathrm{~cm}^{-1}$.

${ }^{1} \mathrm{H}$ NMR (300 MHz, $\left.\mathrm{CDCl}_{3}\right): \delta 7.61(\mathrm{dd}, J=15.8,7.1 \mathrm{~Hz}, 4 \mathrm{H}), 7.47(\mathrm{t}, J=7.4 \mathrm{~Hz}, 2 \mathrm{H}), 7.36$ (t, $J=8.9 \mathrm{~Hz}, 3 \mathrm{H}), 5.98-5.95(\mathrm{~m}, 1 \mathrm{H}), 5.82-5.78(\mathrm{~m}, 1 \mathrm{H}), 3.53-3.48(\mathrm{~m}, 1 \mathrm{H}), 2.15-2.06(\mathrm{~m}$, $3 \mathrm{H}), 1.87-1.59(\mathrm{~m}, 3 \mathrm{H})$.

${ }^{13} \mathrm{C} \mathrm{NMR}\left(75 \mathrm{MHz}, \mathrm{CDCl}_{3}\right): \delta 145.5(\mathrm{C}), 140.8(\mathrm{C}), 138.7(\mathrm{C}), 129.8(\mathrm{CH}), 128.4(2 \mathrm{xCH}), 128.2$ $(\mathrm{CH}), 127.9(2 \times \mathrm{XH}), 126.8(4 \mathrm{xCH}), 126.7(\mathrm{CH}), 41.2(\mathrm{CH}), 32.3\left(\mathrm{CH}_{2}\right), 24.7\left(\mathrm{CH}_{2}\right), 20.9\left(\mathrm{CH}_{2}\right)$. HRMS (EI $\left.{ }^{+}\right) \mathrm{m} / \mathrm{z}$ : [M] ${ }^{+}$Calcd for $\mathrm{C}_{18} \mathrm{H}_{18}$ 234.1408; Found 234.1398.

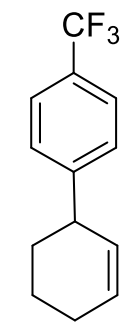


3-(4-Trifluoromethylphenyl)cyclohexene (3d): The compound 3d was prepared according to the general procedure. Purification by flash chromatography in petroleum ether afforded $\mathbf{3 d}$ as clear oil (77 mg, 68\% yield). Exhibited spectra data identical to previous reports. ${ }^{2}$

IR (ATR) $v_{\max }: 2942,1659,1617,1411,1322,1163,1111,1067,1015,830,603 \mathrm{~cm}^{-1}$.

${ }^{1} \mathrm{H}$ NMR (300 MHz, $\mathrm{CDCl}_{3}$ ): $\delta$ 7.57-7.54 (m, 2H), 7.35-7.32 (m, 2H), 5.98-5.92 (m, 1H), 5.72$5.66(\mathrm{~m}, 1 \mathrm{H}), 3.51-3.44(\mathrm{~m}, 1 \mathrm{H}), 2.15-1.99(\mathrm{~m}, 3 \mathrm{H}), 1.80-1.48(\mathrm{~m}, 3 \mathrm{H})$.

${ }^{13} \mathrm{C}$ NMR $\left(75 \mathrm{MHz}, \mathrm{CDCl}_{3}\right): \delta: 150.8\left(\mathrm{C}-\mathrm{F},{ }^{5} J_{\mathrm{C}-\mathrm{F}}=1.3 \mathrm{~Hz}\right), 129.3(\mathrm{CH}), 129.2(\mathrm{CH}), 128.4(\mathrm{C}-$ $\left.\mathrm{F},{ }^{2} J_{\mathrm{C}-\mathrm{F}}=32.2 \mathrm{~Hz}\right), 128.2(2 \mathrm{xCH}), 125.4\left(\mathrm{CH}-\mathrm{F},{ }^{3} J_{\mathrm{C}-\mathrm{F}}=3.8 \mathrm{~Hz}\right), 125.2\left(\mathrm{CH}-\mathrm{F},{ }^{3} J_{\mathrm{C}-\mathrm{F}}=3.8 \mathrm{~Hz}\right)$, $123.5\left(\mathrm{C}-\mathrm{F},{ }^{1} J_{\mathrm{C}-\mathrm{F}}=271 \mathrm{~Hz}\right), 41.9(\mathrm{CH}), 32.6\left(\mathrm{CH}_{2}\right), 25.06\left(\mathrm{CH}_{2}\right), 21.1\left(\mathrm{CH}_{2}\right)$.

${ }^{19} \mathrm{~F}$ NMR $\left(282 \mathrm{MHz}, \mathrm{CDCl}_{3},\right): \delta-62.2$.

HRMS (EI $\left.{ }^{+}\right) \mathrm{m} / \mathrm{z}$ : [M] $]^{+}$Calcd for $\mathrm{C}_{13} \mathrm{H}_{13} \mathrm{~F}_{3}$ 226.0969; Found 226.0963.

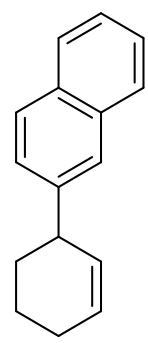

$3 e$

2-(Cyclohex-2-enyl)naphthalene (3e): The compound was prepared according to the general procedure. Purification by flash chromatography in PE/EA (99.5:0.5, v/v) afforded 3e as clear oil (77 mg, 74\% yield). Exhibited spectra data identical to previous reports. ${ }^{5}$

IR (ATR) v $v_{\max }: 3053,3017,2927,2857,1720,1645,1598,1505,1444,1186,952,884,853$, $813,744 \mathrm{~cm}^{-1}$.

${ }^{1} \mathrm{H}$ NMR $\left(300 \mathrm{MHz}, \mathrm{CDCl}_{3}\right.$ ): $\delta$ 7.87-7.82 (m, 3H), 7.70-7.69 (m, 1H), 7.53-7.40 (m, 3H), 6.05$5.98(\mathrm{~m}, 1 \mathrm{H}), 5.90-5.84(\mathrm{~m}, 1 \mathrm{H}), 3.66-3.59(\mathrm{~m}, 1 \mathrm{H}), 2.22-2.10(\mathrm{~m}, 3 \mathrm{H}), 1.88-1.65(\mathrm{~m}, 3 \mathrm{H})$.

${ }^{13} \mathrm{C} \mathrm{NMR}\left(75 \mathrm{MHz}, \mathrm{CDCl}_{3}\right): \delta 144.2(\mathrm{C}), 133.7(\mathrm{C}), 132.3(\mathrm{C}), 130.2(\mathrm{CH}), 128.8(\mathrm{CH}), 128.0$ $(\mathrm{CH}), 127.8(\mathrm{CH}), 127.7(\mathrm{CH}), 126.9(\mathrm{CH}), 126.0(\mathrm{CH}), 125.9(\mathrm{CH}), 125.3(\mathrm{CH}), 42.0(\mathrm{CH})$, $32.6\left(\mathrm{CH}_{2}\right), 25.2\left(\mathrm{CH}_{2}\right), 21.3\left(\mathrm{CH}_{2}\right)$.

HRMS (EI ${ }^{+}$) m/z: $[\mathrm{M}]^{+}$Calcd for $\mathrm{C}_{16} \mathrm{H}_{16}$ 208.1252; Found 208.1250. 


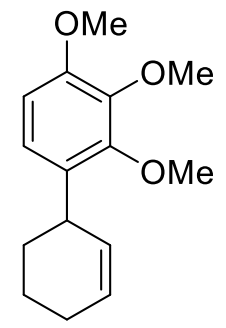

3f

3-(2,3,4-Trimethoxyphenyl)cyclohexene (3f): The compound 3f was prepared according to the general procedure. Purification by flash chromatography in PE/EA (2:98, v/v) afforded $\mathbf{3 f}$ as a clear oil (91 $\mathrm{mg}, 73 \%$ yield).

IR (ATR) $v_{\max }: 2932,2835,1598,1492,1462,1415,1280,1094,1015,907,798,724 \mathrm{~cm}^{-1}$. ${ }^{1} \mathrm{H} \mathrm{NMR}\left(300 \mathrm{MHz}, \mathrm{CDCl}_{3}\right): \delta 6.86(\mathrm{~d}, J=8.6 \mathrm{~Hz}, 1 \mathrm{H}), 6.63(\mathrm{~d}, J=8.6 \mathrm{~Hz}, 1 \mathrm{H}), 5.91-5.84(\mathrm{~m}$, $1 \mathrm{H}), 5.63-5.58(\mathrm{~m}, 1 \mathrm{H}), 3.89(\mathrm{~s}, 3 \mathrm{H}), 3.88(\mathrm{~s}, 3 \mathrm{H}), 3.84(\mathrm{~s}, 3 \mathrm{H}), 3.75-3.70(\mathrm{~m}, 1 \mathrm{H}), 2.10-1.93$ (m, 3H), 1.76-1.43 (m, 3H).

${ }^{13} \mathrm{C}$ NMR (75 MHz, $\left.\mathrm{CDCl}_{3}\right): \delta 152.0(\mathrm{C}), 151.6(\mathrm{C}), 142.2(\mathrm{C}), 132.4(\mathrm{C}), 130.7(\mathrm{CH}), 128.3$ $(\mathrm{CH}), 122.8(\mathrm{CH}), 107.1(\mathrm{CH}), 61.4\left(\mathrm{CH}_{3}\right), 60.8\left(\mathrm{CH}_{3}\right), 56.1\left(\mathrm{CH}_{3}\right), 34.6(\mathrm{CH}), 31.6\left(\mathrm{CH}_{2}\right)$, $25.1\left(\mathrm{CH}_{2}\right), 21.3\left(\mathrm{CH}_{2}\right)$.

HRMS $\left(\mathrm{CI}^{+}\right) \mathrm{m} / \mathrm{z}:[\mathrm{M}+\mathrm{H}]^{+}$Calcd for $\mathrm{C}_{15} \mathrm{H}_{21} \mathrm{O}_{3}$ 249.1490; Found 249.1493.

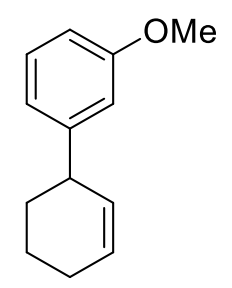

$3 g$

3-(3-Methoxyphenyl)cyclohexene (3g): The compound (3g) was prepared according to the general procedure. Purification by flash chromatography in PE/EA (99:1, v/v) afforded 3g as clear oil (62 mg, 66\% yield). Exhibited spectra data identical to previous reports. ${ }^{4}$ IR (ATR) $v_{\max }$ : 2934, 2835, 1721, 1648, 1599, 1581, 1486, 1452, 1430, 1258, 1205, 1041, 867, $778,697 \mathrm{~cm}^{-1}$.

${ }^{1} \mathrm{H}$ NMR $\left(300 \mathrm{MHz}, \mathrm{CDCl}_{3}\right): \delta$ 7.17-7.11 (m, 1H), 6.75-6.65 (m, 3H), 5.84-5.77 (m, 1H), 5.66$5.60(\mathrm{~m}, 1 \mathrm{H}), 3.72(\mathrm{~s}, 3 \mathrm{H}), 3.35-3.27(\mathrm{~m}, 1 \mathrm{H}), 2.03-1.90(\mathrm{~m}, 3 \mathrm{H}), 1.72-1.42(\mathrm{~m}, 3 \mathrm{H})$.

${ }^{13} \mathrm{C} \mathrm{NMR}\left(75 \mathrm{MHz}, \mathrm{CDCl}_{3}\right): \delta 159.7(\mathrm{C}), 148.6(\mathrm{C}), 130.2(\mathrm{CH}), 129.3(\mathrm{CH}), 128.5(\mathrm{CH}), 120.3$ $(\mathrm{CH}), 113.7(\mathrm{CH}), 111.3(\mathrm{CH}), 55.3\left(\mathrm{CH}_{3}\right), 42.0(\mathrm{CH}), 32.7\left(\mathrm{CH}_{2}\right), 25.1\left(\mathrm{CH}_{2}\right), 21.3\left(\mathrm{CH}_{2}\right)$. HRMS $\left(\mathrm{CI}^{+}\right) \mathrm{m} / \mathrm{z}:[\mathrm{M}+\mathrm{H}]^{+}$Calcd for $\mathrm{C}_{13} \mathrm{H}_{17} \mathrm{O}$ 189.1279; Found: 189.1271. 


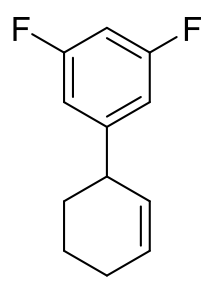

$3 h$

3-(3,5-Difluorophenyl)cyclohexene (3h): The compound $3 \mathrm{~h}$ was prepared according to the general procedure. Purification by flash chromatography in petroleum ether afforded $\mathbf{3 h}$ as clear oil (48 mg, 49\% yield).

IR (ATR) $v_{\max }: 3089,2937,1651,1623,1592,1437,1321,1192,1118,986,852,684 \mathrm{~cm}^{-1}$. ${ }^{1} \mathrm{H}$ NMR $\left(300 \mathrm{MHz}, \mathrm{CDCl}_{3}\right): \delta$ 6.78-6.70 (m, 2H), $6.63(\mathrm{tt}, J=9.0,2.3 \mathrm{~Hz}, 1 \mathrm{H}), 5.96-5.90(\mathrm{~m}$, $1 \mathrm{H})$, 5.68-5.62 (m, 1H), 3.43-3.35 (m, 1H), 2.11-1.96 (m, 3H), 1.77-1.46 (m, 3H).

${ }^{13} \mathrm{C}$ NMR $\left(75 \mathrm{MHz}, \mathrm{CDCl}_{3}\right): \delta 163.2\left(\mathrm{C}-\mathrm{F},{ }^{1} J_{\mathrm{C}-\mathrm{F}}=246 \mathrm{~Hz}\right), 163,0\left(\mathrm{C}-\mathrm{F},{ }^{1} J_{\mathrm{C}-\mathrm{F}}=246 \mathrm{~Hz}\right), 150.9$ $\left(\mathrm{C}-\mathrm{F},{ }^{3} J_{\mathrm{C}-\mathrm{F}}=8.3 \mathrm{~Hz}, \mathrm{C}\right), 129.6(\mathrm{CH}), 128.8(\mathrm{CH}), 110.5\left(2 \mathrm{xCH}-\mathrm{F},{ }^{2} J_{\mathrm{C}-\mathrm{F}}=24.0\right.$ and $\left.{ }^{4} J_{\mathrm{C}-\mathrm{F}}=4.41\right)$, $101.5\left({ }^{2} J_{\mathrm{C}-\mathrm{F},} J=25.2 \mathrm{~Hz},\right), 41.6(\mathrm{CH}), 32.3\left(\mathrm{CH}_{2}\right), 25.0\left(\mathrm{CH}_{2}\right), 20.9\left(\mathrm{CH}_{2}\right)$.

${ }^{19} \mathrm{~F}$ NMR $\left(282 \mathrm{MHz}, \mathrm{CDCl}_{3}\right) \delta-110.7--110.8(\mathrm{~m})$.

HRMS $\left(\mathrm{CI}^{+}\right) \mathrm{m} / \mathrm{z}:[\mathrm{M}+\mathrm{H}]^{+}$Calcd for $\mathrm{C}_{12} \mathrm{H}_{13} \mathrm{~F}_{2}$ 195.0985; Found 195.0993.

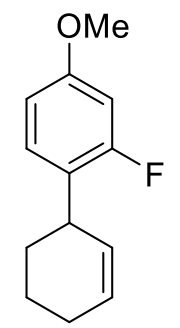

$3 \mathbf{i}$

3-(2-Fluoro-5-methoxyphenyl)cyclohexene (3i): The compound 3i was prepared according to the general procedure. Purification by flash chromatography in PE/EA (99:1, v/v) afforded 3i as clear oil (58 $\mathrm{mg}, 56 \%$ yield).

IR (ATR) $v_{\max }$ : 3023, 2931, 2836, 1623, 1584, 1504, 1443, 1294, 1193, 1151, 1105, 1033, 949, $831,726 \mathrm{~cm}^{-1}$.

${ }^{1} \mathrm{H}$ NMR $\left(300 \mathrm{MHz}, \mathrm{CDCl}_{3}\right): \delta$ 7.14-7.09 (m, 1H), 6.66-6.57 (m, 2H), 5.95-5.88 (m, 1H), 5.66$5.60(\mathrm{~m}, 1 \mathrm{H}), 3.78(\mathrm{~s}, 3 \mathrm{H}), 3.73-3.66(\mathrm{~m}, 1 \mathrm{H}), 2.12-1.94(\mathrm{~m}, 3 \mathrm{H}), 1.75-1.48(\mathrm{~m}, 3 \mathrm{H})$.

${ }^{13} \mathrm{C} \mathrm{NMR}\left(75 \mathrm{MHz}, \mathrm{CDCl}_{3}\right): \delta 161.1\left(\mathrm{C}-\mathrm{F},{ }^{1} J_{\mathrm{C}-\mathrm{F}}=245 \mathrm{~Hz}\right), 159.0\left(\mathrm{C}-\mathrm{F},{ }^{3} J_{\mathrm{C}-\mathrm{F}}=11.0 \mathrm{~Hz}\right), 129.6$ $\left(\mathrm{CH}-\mathrm{F},{ }^{3} J_{\mathrm{C}-\mathrm{F}}=6.8 \mathrm{~Hz}\right), 129.3(\mathrm{CH}), 128.9(\mathrm{CH}), 124.9\left(\mathrm{C}-\mathrm{F},{ }^{2} J_{\mathrm{C}-\mathrm{F}}=15.2 \mathrm{~Hz}\right), 109.3(\mathrm{CH}-\mathrm{F}$, 
$\left.{ }^{4} J_{\mathrm{C}-\mathrm{F}}=3.1 \mathrm{~Hz}\right), 101.5\left(\mathrm{CH}-\mathrm{F},{ }^{2} J_{\mathrm{C}-\mathrm{F}}=26.3 \mathrm{~Hz}, \mathrm{CH}\right), 55.5\left(\mathrm{CH}_{3}\right), 33.7\left(\mathrm{CH}-\mathrm{F},{ }^{3} J_{\mathrm{C}-\mathrm{F}}=2.1 \mathrm{~Hz}\right)$, $30.7\left(\mathrm{CH}_{2}\right), 24.9\left(\mathrm{CH}_{2}\right), 20.8\left(\mathrm{CH}_{2}\right)$.

${ }^{19} \mathrm{~F} \mathrm{NMR}\left(282 \mathrm{MHz}, \mathrm{CDCl}_{3}\right) \delta-117.8(\mathrm{~d}, J=20.7$ and $2.8 \mathrm{~Hz})$;

HRMS (EI $\left.{ }^{+}\right) \mathrm{m} / \mathrm{z}$ : [M] ${ }^{+}$Calcd for $\mathrm{C}_{13} \mathrm{H}_{15} \mathrm{FO}$ 206.1106; Found 206.1108.

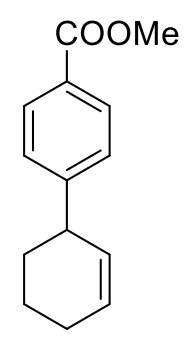

3j

Methyl-4-(Cyclohex-2-en-1-yl)-benzoate (3j): The compound 3j was prepared according to the general procedure. Purification by flash chromatography in PE/EA (98:2, v/v) afforded 3j as clear oil (66 mg, 61\% yield). Exhibited spectra data identical to previous reports. ${ }^{6}$ IR (ATR) v $v_{\max }: 2948,2857,1717,1607,1435,1411,1275,1186,1104,1017,959,853,768$, $703 \mathrm{~cm}^{-1}$.

${ }^{1} \mathrm{H}$ NMR $\left(300 \mathrm{MHz}, \mathrm{CDCl}_{3}\right): \delta$ 7.90-7.86 (m, 2H), 7.22-7.18 (m, 2H), 5.78-5.71 (m, 1H), 5.54$5.48(\mathrm{~m}, 1 \mathrm{H}), 3.72(\mathrm{~s}, 3 \mathrm{H}), 3.31-3.25(\mathrm{~m}, 1 \mathrm{H}), 1.93-1.79(\mathrm{~m}, 3 \mathrm{H}), 1.60-1.30$ (m, 3H).

${ }^{13} \mathrm{C}$ NMR (75 MHz, $\left.\mathrm{CDCl}_{3}\right): \delta 167.3(\mathrm{C}), 152.2(\mathrm{C}), 129.8(2 \mathrm{xCH}), 129.3(\mathrm{CH}), 129.1(\mathrm{CH})$, $128.0(\mathrm{C}), 127.8(2 \mathrm{xCH}), 52.1\left(\mathrm{CH}_{3}\right), 41.9(\mathrm{CH}), 32.5\left(\mathrm{CH}_{2}\right), 25.0\left(\mathrm{CH}_{2}\right), 21.1\left(\mathrm{CH}_{2}\right)$.

HRMS $\left(\mathrm{CI}^{+}\right) \mathrm{m} / \mathrm{z}:[\mathrm{M}+\mathrm{H}]^{+}$Calcd for $\mathrm{C}_{14} \mathrm{H}_{17} \mathrm{O}_{2} 217.1228$; Found 217.1222.<smiles>CC(=O)c1ccc(C2C=CCCC2)cc1</smiles>

$3 \mathbf{k}$

3-(4-Acetylpheny)cyclohexene (3k): The compound 3k was prepared according to the general procedure. Purification by flash chromatography in PE/EA (97:3, v/v) afforded 3k was clear oil (71 mg, 70\% yield). Exhibited spectra data identical to previous reports. ${ }^{7}$ IR (ATR) $v_{\max }$ 2931, 2853, 1680, 1603, 1561, 1413, 1358, 1268, 1182, 1072, 1015, 959, 828, $732,596 \mathrm{~cm}^{-1}$. 
${ }^{1} \mathrm{H}$ NMR (300 MHz, $\left.\mathrm{CDCl}_{3}\right): \delta$ 7.91-7.87 (m, 2H), 7.32-7.28 (m, 2H), 5.96-5.89 (m, 1H), 5.71-5.65 (m, 1H), 3.49-3.42 (m, 1H), 2.58 (s, 3H), 2.11-1.97 (m, 3H), 1.79-1.47 (m, 3H). ${ }^{13} \mathrm{C}$ NMR (75 MHz, $\left.\mathrm{CDCl}_{3}\right): \delta 198.0(\mathrm{C}), 152.5(\mathrm{C}), 135.3(\mathrm{C}), 129.2(2 \mathrm{xCH}), 128.6(2 \mathrm{xCH})$, $128.0(2 \times C H), 41.9(\mathrm{CH}), 32.4\left(\mathrm{CH}_{2}\right), 26.7\left(\mathrm{CH}_{3}\right), 25.0\left(\mathrm{CH}_{2}\right), 21.1\left(\mathrm{CH}_{2}\right)$.

HRMS (EI ${ }^{+}$) m/z: [M] $]^{+}$Calcd for $\mathrm{C}_{14} \mathrm{H}_{16} \mathrm{O}$ 200.1201; Found 200.1206.

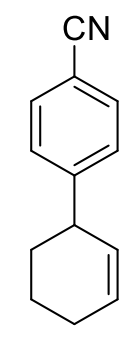

31

3-(4-Cyanophenyl)cyclohexene (31): The compound 31 was prepared according to the general procedure. Purification by flash chromatography in PE/EA (97:3, v/v) afforded 31 as clear oil (67 mg, 73\% yield). Exhibited spectra data identical to previous reports. ${ }^{8}$

IR (ATR) vmax: 2931, 2859, 2227, 1718, 1650, 1605, 1504, 1407, 1328, 1262, 1190, 1017, $956,824,565 \mathrm{~cm}^{-1}$.

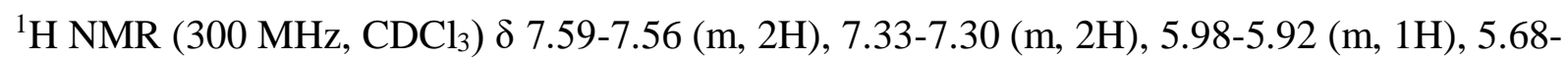
$5.62(\mathrm{~m}, 1 \mathrm{H}), 3.49-3.42(\mathrm{~m}, 1 \mathrm{H}), 2.12-1.97(\mathrm{~m}, 3 \mathrm{H}), 1.77-1.45(\mathrm{~m}, 3 \mathrm{H})$.

${ }^{13} \mathrm{C}$ NMR $\left(75 \mathrm{MHz}, \mathrm{CDCl}_{3}\right) \delta: 152.3(\mathrm{C}), 132.2(2 \mathrm{xCH}), 129.7(\mathrm{CH}), 128.7(2 \mathrm{xCH}), 128.5$ $(\mathrm{CH}), 119.3(\mathrm{C}), 109.9(\mathrm{C}), 42.0(\mathrm{CH}), 32.4\left(\mathrm{CH}_{2}\right), 24.9\left(\mathrm{CH}_{2}\right), 20.9\left(\mathrm{CH}_{2}\right)$.

HRMS (EI $\left.{ }^{+}\right) \mathrm{m} / \mathrm{z}$ : [M] ${ }^{+}$Calcd for $\mathrm{C}_{13} \mathrm{H}_{13} \mathrm{~N}$ 183.1048; Found 183.1048.

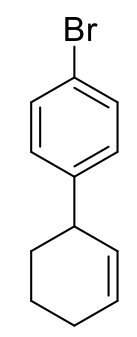

3m

3-(4-Bromophenyl)cyclohexene (3m): The compound 3m was prepared according to the general procedure. Purification by flash chromatography in PE/EA (99:1, v/v) afforded $\mathbf{3 m}$ as clear oil (68 mg, 57\% yield). Exhibited spectra data identical to previous reports. ${ }^{3}$

IR (ATR) $v_{\max }$ : 2930, 1720, 1665, 1584, 1487, 1397, 1327, $12601190,1070,1008,812 \mathrm{~cm}^{-1}$. 
${ }^{1} \mathrm{H}$ NMR $\left(300 \mathrm{MHz}, \mathrm{CDCl}_{3}\right): \delta$ 7.43-7.39 (m, 2H), 7.12-7.07 (m, 2H), 5.94-5.87 (m, 1H), 5.69$5.63(\mathrm{~m}, 1 \mathrm{H}), 3.40-3.34(\mathrm{~m}, 1 \mathrm{H}), 2.11-1.95(\mathrm{~m}, 3 \mathrm{H}), 1.78-1.45(\mathrm{~m}, 3 \mathrm{H})$.

${ }^{13} \mathrm{C}$ NMR (75 MHz, $\left.\mathrm{CDCl}_{3}\right): \delta 145.8(\mathrm{C}), 131.4(2 \mathrm{xCH}), 129.7(2 \mathrm{xCH}), 129.6(\mathrm{CH}), 129.0$ $(\mathrm{CH}), 119.8(\mathrm{C}), 41.4(\mathrm{CH}), 32.6\left(\mathrm{CH}_{2}\right), 25.0\left(\mathrm{CH}_{2}\right), 21.1\left(\mathrm{CH}_{2}\right)$.

HRMS (EI $\left.{ }^{+}\right) \mathrm{m} / \mathrm{z}$ : [M] $]^{+}$Calcd for $\mathrm{C}_{12} \mathrm{H}_{13} \mathrm{Br}$ 236.0200; Found 236.0200.

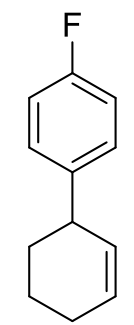

$3 n$

3-(4-Fluorophenyl)cyclohexene (3n): The compound 3n was prepared according to the general procedure. Purification by flash chromatography in PE/EA (99:1, v/v) afforded 3n as clear oil (53 mg, 60\% yield). Exhibited spectra data identical to previous reports. ${ }^{1}$ IR (ATR) $v_{\max }$ : 2931, 1721, 1657, 1599, 1508, 1411, 1224, 1159, 1064, 1013, 878, $829 \mathrm{~cm}^{-1}$. ${ }^{1} \mathrm{H}$ NMR $\left(300 \mathrm{MHz}, \mathrm{CDCl}_{3}\right): \delta$ 7.20-7.14 (m, 2H), 7.02-6.94 (m, 2H), 5.93-5.86 (m, 1H), 5.71-5.65 (m, 1H), 3.43-3.36 (m, 1H), 2.12-1.95 (m, 3H), 1.78-1.46 (m, 3H).

${ }^{13} \mathrm{C} \mathrm{NMR}\left(75 \mathrm{MHz}, \mathrm{CDCl}_{3}\right): \delta 161.4\left(\mathrm{C}-\mathrm{F},{ }^{1} J_{\mathrm{C}-\mathrm{F}}=243.4 \mathrm{~Hz}\right), 142.4\left(\mathrm{C}-\mathrm{F},{ }^{4} J_{\mathrm{C}-\mathrm{F}}=3.1 \mathrm{~Hz}\right), 130.1$ $(\mathrm{CH}), 129.2\left(2 \mathrm{xCH}-\mathrm{F},{ }^{3} J_{\mathrm{C}-\mathrm{F}}=7.8 \mathrm{~Hz}\right), 128.7(\mathrm{CH}), 115.1\left(2 \mathrm{xCH}-\mathrm{F},{ }^{2} J_{\mathrm{C}-\mathrm{F}}=21.0 \mathrm{~Hz}\right), 41.2(\mathrm{CH})$, $32.9\left(\mathrm{CH}_{2}\right), 25.1\left(\mathrm{CH}_{2}\right), 21.1\left(\mathrm{CH}_{2}\right)$.

${ }^{19} \mathrm{~F}$ NMR $\left(282 \mathrm{MHz}, \mathrm{CDCl}_{3}\right): \delta-117.6--117.7(\mathrm{~m})$

HRMS $\left(\mathrm{EI}^{+}\right) \mathrm{m} / \mathrm{z}$ : $[\mathrm{M}]^{+}$Calcd for $\mathrm{C}_{12} \mathrm{H}_{13} \mathrm{~F}$ 176.1001; Found 176.1002.

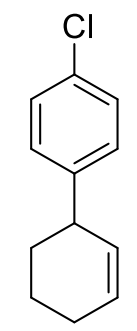

30

3-(4-Chlorophenyl)cyclohexene (3o): The compound 3o was prepared according to the general procedure. Purification by flash chromatography in PE/EA (99:1, v/v) afforded 3o as clear oil (51 mg, 53\% yield). Exhibited spectra data identical to previous reports. ${ }^{5}$ 
IR (ATR) $v_{\max }: 3010,2930,1710,1680,1574,1517,1323,1220,1185,1065,1008,812 \mathrm{~cm}^{-1}$. ${ }^{1} \mathrm{H}$ NMR $\left(300 \mathrm{MHz}, \mathrm{CDCl}_{3}\right): \delta$ 7.28-7.24 (m, 2H), 7.17-7.13 (m, 2H), 5.94-5.87 (m, 1H), 5.70$5.64(\mathrm{~m}, 1 \mathrm{H}), 3.42-3.35(\mathrm{~m}, 1 \mathrm{H}), 2.12-1.95(\mathrm{~m}, 3 \mathrm{H}), 1.78-1.45(\mathrm{~m}, 3 \mathrm{H})$.

${ }^{13} \mathrm{C}$ NMR (75 MHz, $\left.\mathrm{CDCl}_{3}\right): \delta 145.2(\mathrm{C}), 131.7(\mathrm{C}), 129.7(\mathrm{CH}), 129.2(2 \times \mathrm{CH}), 128.9(\mathrm{CH})$, $128.5(2 x \mathrm{CH}), 41.3(\mathrm{CH}), 32.7\left(\mathrm{CH}_{2}\right), 25.1\left(\mathrm{CH}_{2}\right), 21.1\left(\mathrm{CH}_{2}\right)$.

HRMS (EI $\left.{ }^{+}\right) \mathrm{m} / \mathrm{z}$ : [M] ${ }^{+}$Calcd for $\mathrm{C}_{12} \mathrm{H}_{13} \mathrm{Cl}$ 192.0705; Found 192.0709.<smiles>O=[N+]([O-])c1ccc(C2C=CCCC2)cc1</smiles>

$3 \mathbf{p}$

3-(4-nitrophenyl)cyclohexene (3p): The compound 3p was prepared according to the general procedure. Purification by flash chromatography in PE/EA (96:4, v/v) afforded $\mathbf{3 p}$ as orange oil (37 mg, 36\% yield).

IR (ATR) vmax: 3022, 2929, 2858, 1596, 1513, 1446, 1342, 1108, 1014, 985, 850, 840, 752, $698 \mathrm{~cm}^{-1}$.

${ }^{1} \mathrm{H}$ NMR $\left(300 \mathrm{MHz}, \mathrm{CDCl}_{3}\right) \delta 8.17-8.12(\mathrm{~m}, 2 \mathrm{H}), 7.39-7.34(\mathrm{~m}, 2 \mathrm{H}), 6.00-5.94(\mathrm{~m}, 1 \mathrm{H}), 5.70-$ $5.64(\mathrm{~m}, 1 \mathrm{H}), 3.56-3.48$ (m, 1H), 2.14-1.99 (m, 3H), 1.79-1.47 (m, 3H).

${ }^{13} \mathrm{C}$ NMR (75 MHz, $\left.\mathrm{CDCl}_{3}\right) \delta ; 154.6(\mathrm{C}), 146.5(\mathrm{C}), 129.9(\mathrm{CH}), 128.6(2 \mathrm{xCH}), 128.4(\mathrm{CH})$, $123.7(2 \times \mathrm{CH}), 41.9(\mathrm{CH}), 32.4\left(\mathrm{CH}_{2}\right), 24.9\left(\mathrm{CH}_{2}\right), 20.9\left(\mathrm{CH}_{2}\right)$.

HRMS (EI $\left.{ }^{+}\right) \mathrm{m} / \mathrm{z}:[\mathrm{M}]^{+}$Calcd for $\mathrm{C}_{12} \mathrm{H}_{13} \mathrm{NO}_{2}$ 203.0946; Found 203.0949.

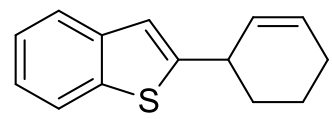

$3 q$

2-(cyclohex-2-enyl)benzo[b]thiophene (3q): The compound 3q was prepared according to the general procedure. Purification by flash chromatography in petroleum ether afforded $\mathbf{3 q}$ as light-yellow oil (56 mg, 52\% yield). Exhibited spectra data identical to previous reports. ${ }^{9}$ IR (ATR) $v_{\max }: 3055,2933,1719,1648,1434,1156,1067,824,745,725 \mathrm{~cm}^{-1}$. 
${ }^{1} \mathrm{H}$ NMR $\left(300 \mathrm{MHz}, \mathrm{CDCl}_{3}\right): \delta$ 7.69-7.66 (m, 1H), 7.59-7.56 (m, 1H), 7.23-7.12 (m, 2H), 6.95 $(\mathrm{s}, 1 \mathrm{H}), 5.85-5.67(\mathrm{~m}, 2 \mathrm{H}), 3.66-3.61(\mathrm{~m}, 1 \mathrm{H}), 2.02-1.98(\mathrm{~m}, 3 \mathrm{H}), 1.76-1.66(\mathrm{~m}, 2 \mathrm{H}), 1.61-1.52$ (m, 1H).

${ }^{13} \mathrm{C}$ NMR (75MHz, $\left.\mathrm{CDCl}_{3}\right): \delta 151.3(\mathrm{C}), 140.1(\mathrm{C}), 139.3(\mathrm{C}), 129.1(\mathrm{CH}), 129.0(\mathrm{CH}), 124.1$ $(\mathrm{CH}), 123.5(\mathrm{CH}), 122.9(\mathrm{CH}), 122.3(\mathrm{CH}), 120.2(\mathrm{CH}), 37.4(\mathrm{CH}), 32.0\left(\mathrm{CH}_{2}\right), 25.1\left(\mathrm{CH}_{2}\right)$, $20.7\left(\mathrm{CH}_{2}\right)$.

HRMS $\left(\mathrm{EI}^{+}\right) \mathrm{m} / \mathrm{z}:[\mathrm{M}]^{+}$Calcd for $\mathrm{C}_{14} \mathrm{H}_{14} \mathrm{~S}$ 214.0816; found 214.0812.

2. Substrate scope of boronic acids with cycloheptene 4:

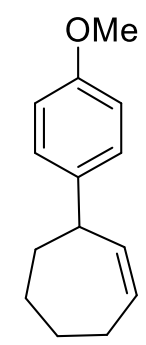

12a

3-(4-Methoxyphenyl)cycloheptene (12a): The compound 12a was prepared according to the general procedure. Purification by flash chromatography in PE/EA (99:1, v/v) afforded 12a as clear oil (71 mg, 70\% yield). Exhibited spectra data identical to previous reports. ${ }^{11}$

IR (ATR) $v_{\max }: 3014,2918,2835,1610,1583,1509,1441,1301,1242,1176,1035,899,824$, $685 \mathrm{~cm}^{-1}$.

${ }^{1} \mathrm{H}$ NMR $\left(300 \mathrm{MHz}, \mathrm{CDCl}_{3}\right): \delta$ 7.20-7.15 (m, 2H), 6.90-6.85 (m, 2H), 5.92-5.83 (m, 1H), 5.80$5.75(\mathrm{~m}, 1 \mathrm{H}), 3.81(\mathrm{~s}, 3 \mathrm{H}), 3.54-3.51(\mathrm{~m}, 1 \mathrm{H}), 2.29-2.22(\mathrm{~m}, 2 \mathrm{H}), 1.98-1.91(\mathrm{~m}, 1 \mathrm{H}), 1.87-1.62$ (m, 4H), 1.54-1.42 (m, 1H).

${ }^{13} \mathrm{C}$ NMR (75 MHz, $\left.\mathrm{CDCl}_{3}\right): \delta 157.8(\mathrm{C}), 140.2(\mathrm{C}), 137.5(\mathrm{CH}), 131.5(\mathrm{CH}), 128.2(2 \mathrm{xCH})$, $113.8(2 x \mathrm{CH}), 55.3\left(\mathrm{CH}_{3}\right), 46.4(\mathrm{CH}), 36.5\left(\mathrm{CH}_{2}\right), 30.2\left(\mathrm{CH}_{2}\right), 28.9\left(\mathrm{CH}_{2}\right), 27.1\left(\mathrm{CH}_{2}\right)$.

HRMS (EI $\left.{ }^{+}\right) \mathrm{m} / \mathrm{z}:[\mathrm{M}]^{+}$Calcd for $\mathrm{C}_{14} \mathrm{H}_{18} \mathrm{O}$ 202.1357; Found 202.1365.

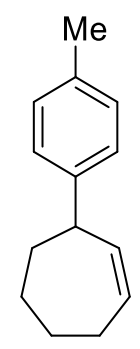


3-(Tolyl)cycloheptene (12b): The compound 12b was prepared according to the general procedure. Purification by flash chromatography in petroleum ether afforded $\mathbf{1 2 b}$ as clear oil (72 mg, 77\% yield). Exhibited spectra data identical to previous reports. ${ }^{12}$ IR (ATR) $v_{\max }: 2922,2858,1722,1682,1606,1513,1445,1179,1020,842,808 \mathrm{~cm}^{-1}$. ${ }^{1} \mathrm{H}$ NMR (300 MHz, $\left.\mathrm{CDCl}_{3}\right): \delta 7.15$ (s, 4H), 5.92-5.84 (m, 1H), 5.81-5.76 (m, 1H), 3.55-3.51 (m, 1H), $2.36(\mathrm{~s}, 3 \mathrm{H}), 2.30-2.20(\mathrm{~m}, 2 \mathrm{H}), 2.02-1.92(\mathrm{~m}, 1 \mathrm{H}), 1.90-1.62(\mathrm{~m}, 4 \mathrm{H}), 1.53-1.42(\mathrm{~m}$, $1 \mathrm{H})$.

${ }^{13} \mathrm{C}$ NMR (75 MHz, $\left.\mathrm{CDCl}_{3}\right): \delta$ 145.1(C), $137.5(\mathrm{CH}), 135.3(\mathrm{C}), 131.6(\mathrm{CH}), 129.2(2 \times \mathrm{CH})$, 127.2 $(2 x \mathrm{xH}), 46.9(\mathrm{CH}), 36.5\left(\mathrm{CH}_{2}\right), 30.4\left(\mathrm{CH}_{2}\right), 28.9\left(\mathrm{CH}_{2}\right), 27.2\left(\mathrm{CH}_{2}\right), 21.0\left(\mathrm{CH}_{3}\right)$.

HRMS (EI ${ }^{+}$m/z: [M] ${ }^{+}$Calcd for $\mathrm{C}_{14} \mathrm{H}_{18}$ 186.1408; Found 186.1396.

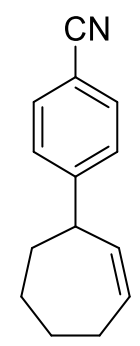

121

3-(4-Cyanophenyl)cycloheptene (12l): The compound $12 \mathrm{l}$ was prepared according to the general procedure. Purification by flash chromatography in PE/EA (99:1, v/v) afforded $\mathbf{1 2 l}$ as clear oil (70 mg, 75\% yield). ). Exhibited spectra data identical to previous reports. ${ }^{8}$ IR (ATR) $v_{\max }: 3018,2922,2852,2226,1647,1606,1503,1444,1176,1017,898,829,685$ $\mathrm{cm}^{-1}$.

${ }^{1} \mathrm{H}$ NMR (300 MHz, $\left.\mathrm{CDCl}_{3}\right) \delta$ 7.60-7.55 (m, 2H), 7.34-7.30 (m, 2H), 5.94-5.85 (m, 1H), 5.68$5.62(\mathrm{~m}, 1 \mathrm{H}), 3.59-3.57(\mathrm{~m}, 1 \mathrm{H}), 2.28-2.18(\mathrm{~m}, 2 \mathrm{H}), 1.94-1.60(\mathrm{~m}, 5 \mathrm{H}), 1.51-1.39(\mathrm{~m}, 1 \mathrm{H})$. ${ }^{13} \mathrm{C}$ NMR $\left(75 \mathrm{MHz}, \mathrm{CDCl}_{3}\right) \delta ; 153.2(\mathrm{C}), 135.1(\mathrm{CH}), 133.0(\mathrm{CH}), 132.4(2 \mathrm{xCH}), 128.2$ (2xCH), $119.2(\mathrm{C}), 109.8(\mathrm{C}), 47.2(\mathrm{CH}), 35.9\left(\mathrm{CH}_{2}\right), 30.0\left(\mathrm{CH}_{2}\right), 28.8\left(\mathrm{CH}_{2}\right), 26.9\left(\mathrm{CH}_{2}\right)$. HRMS (EI $\left.{ }^{+}\right) \mathrm{m} / \mathrm{z}:[\mathrm{M}]^{+}$Calcd for $\mathrm{C}_{14} \mathrm{H}_{15} \mathrm{~N}$ 197.1204; Found 197.1204.

3. Substrate scope of boronic acids with cyclooctene $\mathbf{5}$ : 


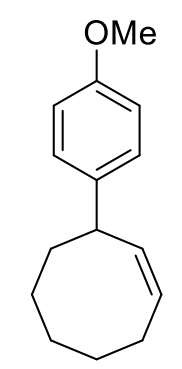

13a

3-(4-Methoxyphenyl)cyclooctene (13a): The compound 13a was prepared according to the general procedure. Purification by flash chromatography in PE/EA (99:1, v/v) afforded 13a as colorless oil (67 mg, 62\% yield). Exhibited spectra data identical to previous reports. ${ }^{13}$ IR (ATR) $v_{\max }: 3012,2923,2851,1611,1510,1442,1244,1177,1037,821 \mathrm{~cm}^{-1}$. ${ }^{1} \mathrm{H}$ NMR (300MHz, $\left.\mathrm{CDCl}_{3}\right): \delta$ 7.24-7.19 (m, 2H), 6.90-6.85 (m, 2H), 5.76-5.67 (m, 1H), 5.62$5.56(\mathrm{~m}, 1 \mathrm{H}), 3.81(\mathrm{~s}, 3 \mathrm{H}), 3.78-3.70(\mathrm{~m}, 1 \mathrm{H}), 2.46-234(\mathrm{~m}, 1 \mathrm{H}), 2.20-2.12(\mathrm{~m}, 1 \mathrm{H}), 1.87-1.39$ $(\mathrm{m}, 8 \mathrm{H})$.

${ }^{13} \mathrm{C}$ NMR $\left(75 \mathrm{MHz}, \mathrm{CDCl}_{3}\right): \delta 157.8(\mathrm{C}), 138.8(\mathrm{C}), 134.6(\mathrm{CH}), 128.9(\mathrm{CH}), 128.2(2 \times \mathrm{CH})$, $113.9(2 \times \mathrm{XH}), 55.3\left(\mathrm{CH}_{3}\right), 41.4(\mathrm{CH}), 37.8\left(\mathrm{CH}_{2}\right), 29.8\left(\mathrm{CH}_{2}\right), 26.8\left(\mathrm{CH}_{2}\right), 26.6\left(\mathrm{CH}_{2}\right), 26.2$ $\left(\mathrm{CH}_{2}\right)$.

HRMS (EI ${ }^{+}$) m/z: [M] $]^{+}$Calcd for $\mathrm{C}_{15} \mathrm{H}_{20} \mathrm{O}$ 216.1514; Found 216.1515.

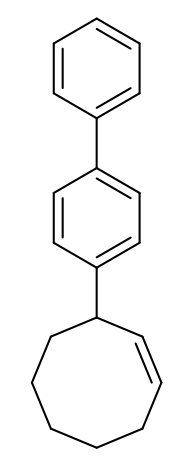

$13 \mathrm{c}$

3-(1,1'Biphenyl)cyclooctene (13c): The compound 13c was prepared according to the general procedure. Purification by flash chromatography in Petroleum ether afforded $\mathbf{1 3 c}$ as clear oil (76 mg, 58\% yield). Exhibited spectra data identical to previous reports. ${ }^{13}$

IR (ATR) $v_{\max }: 3017,2918,2848,1598,1524,1487,1447,1129,1005,827,753,733,690 \mathrm{~cm}^{-}$ 1 . 
${ }^{1} \mathrm{H}$ NMR $\left(300 \mathrm{MHz}, \mathrm{CDCl}_{3}\right): \delta$ 7.62-7.53 (m, 4H), 7.47-7.41 (m, 2H), 7.38-7.31 (m, 3H), 5.80$5.62(\mathrm{~m}, 2 \mathrm{H}), 3.87-3.78(\mathrm{~m}, 1 \mathrm{H}), 2.48-2.37(\mathrm{~m}, 1 \mathrm{H}), 2.22-2.13(\mathrm{~m}, 1 \mathrm{H}), 1.97-1.88(\mathrm{~m}, 1 \mathrm{H})$, 1.84-1.65 (m, 5H), 1.59-1.35 (m, 2H).

${ }^{13} \mathrm{C} \mathrm{NMR}\left(75 \mathrm{MHz}, \mathrm{CDCl}_{3}\right): \delta 145.8(\mathrm{C}), 141.2(\mathrm{C}), 138.9(\mathrm{C}), 134.1(\mathrm{CH}), 129.3(\mathrm{CH}), 128.8$ $(2 \mathrm{xCH}), 127.8(2 \mathrm{xCH}), 127.3(2 \mathrm{xCH}), 127.2(2 \mathrm{xCH}), 127.1(\mathrm{CH}), 42.1(\mathrm{CH}), 37.6\left(\mathrm{CH}_{2}\right), 29.8$ $\left(\mathrm{CH}_{2}\right), 26.9\left(\mathrm{CH}_{2}\right), 26.7\left(\mathrm{CH}_{2}\right), 26.2\left(\mathrm{CH}_{2}\right)$.

HRMS (EI $\left.{ }^{+}\right) \mathrm{m} / \mathrm{z}$ : [M] $]^{+}$Calcd for $\mathrm{C}_{20} \mathrm{H}_{22}$ 262.1721; Found 262.1722.

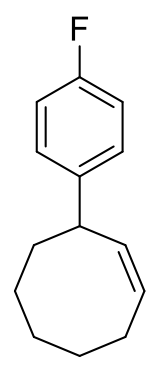

13n

3-(4-Fluorophenyl)cyclooctene (13n): The compound 13n was prepared according to the general procedure. Purification by flash chromatography in petroleum ether afforded $\mathbf{1 3 n}$ as colorless oil (46 mg, $45 \%$ yield).

IR (ATR) $v_{\max }: 3016,2926,2853,1604,1508,1450,1223,1158,825 \mathrm{~cm}^{-1}$.

${ }^{1} \mathrm{H}$ NMR $\left(300 \mathrm{MHz}, \mathrm{CDCl}_{3}\right): \delta$ 7.24-7.20 (m, 2H), 7.01-6.95 (m, 2H), 5.75-5.66 (m, 1H), 5.57$5.50(\mathrm{~m}, 1 \mathrm{H}), 3.78-3.69(\mathrm{~m}, 1 \mathrm{H}), 2.42-2.30(\mathrm{~m}, 1 \mathrm{H}), 2.17-2.13(\mathrm{~m}, 1 \mathrm{H}), 1.84-1.36(\mathrm{~m}, 8 \mathrm{H})$.

${ }^{13} \mathrm{C} \mathrm{NMR}\left(75 \mathrm{MHz}, \mathrm{CDCl}_{3}\right): \delta 161.3\left(\mathrm{C}-\mathrm{F},{ }^{1} J_{\mathrm{C}-\mathrm{F}}=243.4 \mathrm{~Hz}\right), 142.3\left(\mathrm{C}-\mathrm{F},{ }^{4} J_{\mathrm{C}-\mathrm{F}}=3.2 \mathrm{~Hz}\right), 134.1$ $(\mathrm{CH}), 129.3(\mathrm{CH}), 128.7\left(2 \mathrm{xCH}-\mathrm{F},{ }^{3} J_{\mathrm{C}-\mathrm{F}}=7.7 \mathrm{~Hz}\right), 115.2\left(2 \mathrm{xCH}-\mathrm{F},{ }^{2} J_{\mathrm{C}-\mathrm{F}}=21.0 \mathrm{~Hz}\right), 41.6(\mathrm{CH})$, $37.7\left(\mathrm{CH}_{2}\right), 29.7\left(\mathrm{CH}_{2}\right), 26.8\left(\mathrm{CH}_{2}\right), 26.6\left(\mathrm{CH}_{2}\right), 26.1\left(\mathrm{CH}_{2}\right)$.

${ }^{19} \mathrm{~F}$ NMR $\left(\mathrm{MHz}, \mathrm{CDCl}_{3}\right): \delta-117.74--117.8(\mathrm{~m})$.

HRMS (EI ${ }^{+}$) m/z: [M] ${ }^{+}$Calcd for $\mathrm{C}_{14} \mathrm{H}_{17} \mathrm{~F}$ 204.1314; Found 204.1310.

4. Substrate scope of boronic acids with cyclopentene $\mathbf{6}$ :

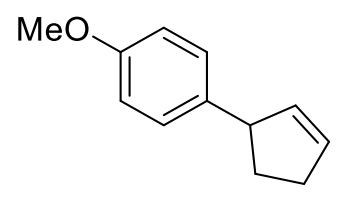

$14 a$ 
3-(4-Methoxyphenyl)cyclopentene (14a) The compound 14a was prepared according to the general procedure. Purification by flash chromatography in petroleum ether afforded $\mathbf{1 4 a}$ as clear oil (35 mg, 40\% yield). Exhibited spectra data identical to previous reports. ${ }^{10}$ IR (ATR) $v_{\max }: 2934,2838,1673,1607,1510,1462,1301,1245,1177,1033,828 \mathrm{~cm}^{-1}$. ${ }^{1} \mathrm{H}$ NMR $\left(300 \mathrm{MHz}, \mathrm{CDCl}_{3}\right): \delta$ 7.13-7.09 (m, 2H), 6.86-6.81 (m, 2H), 5.94-5.89 (m, 1H), 5.77$5.73(\mathrm{~m}, 1 \mathrm{H}), 3.89-3.81(\mathrm{~m}, 1 \mathrm{H}), 3.79(\mathrm{~s}, 3 \mathrm{H}), 2.53-2.32(\mathrm{~m}, 3 \mathrm{H}), 1.73-1.63(\mathrm{~m}, 1 \mathrm{H})$.

${ }^{13} \mathrm{C} \mathrm{NMR}\left(75 \mathrm{MHz}, \mathrm{CDCl}_{3}\right): \delta 157.9(\mathrm{C}), 138.7(\mathrm{C}), 134.6(\mathrm{CH}), 131.6(\mathrm{CH}), 128.1(2 \mathrm{xCH})$, $113.8(2 \times C H), 55.3\left(\mathrm{CH}_{3}\right), 50.5(\mathrm{CH}), 33.9\left(\mathrm{CH}_{2}\right), 32.4\left(\mathrm{CH}_{2}\right)$.

HRMS (EI ${ }^{+}$m/z: [M] ${ }^{+}$Calcd for $\mathrm{C}_{12} \mathrm{H}_{14} \mathrm{O}$ 174.1044; Found 174.1052.

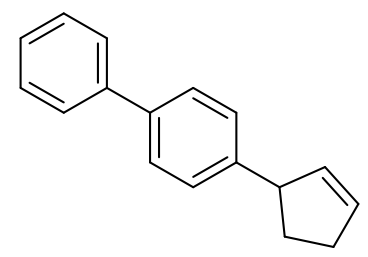

$14 \mathrm{c}$

3-(1,1'-Biphenyl)cyclopentene (14c): The compound 14c was prepared according to the general procedure. Purification by flash chromatography in Petroleum ether afforded $\mathbf{1 4 c}$ as colorless oil (42 mg, 38\% yield). Exhibited spectra data identical to previous reports. ${ }^{10}$

IR (ATR) $v_{\max }: 3034,2920,1671,1485,1335,1035,840,760,688 \mathrm{~cm}^{-1}$.

${ }^{1} \mathrm{H}$ NMR $\left(300 \mathrm{MHz}, \mathrm{CDCl}_{3}\right): \delta$ 7.65-757 (m, 4H), 7.50-7.45 (m, 2H), 7.40-7.31 (m, 3H), 6.04$6.01(\mathrm{~m}, 1 \mathrm{H}), 5.88-5.86(\mathrm{~m}, 1 \mathrm{H}), 4.00-3.99(\mathrm{~m}, 1 \mathrm{H}), 2.60-2.46(\mathrm{~m}, 3 \mathrm{H}), 1.88-1.78(\mathrm{~m}, 1 \mathrm{H})$.

${ }^{13} \mathrm{C}$ NMR (75MHz, $\left.\mathrm{CDCl}_{3}\right): \delta 145.7(\mathrm{C}), 141.2(\mathrm{C}), 139.1(\mathrm{C}), 134.3(\mathrm{CH}), 132.1(\mathrm{CH}), 128.8$ $(2 \mathrm{xCH}), 127.7(2 \mathrm{xCH}), 127.2(2 \mathrm{xCH}), 127.1(2 \mathrm{xCH}), 127.1(\mathrm{CH}), 51.1(\mathrm{CH}), 33.9\left(\mathrm{CH}_{2}\right), 32.6$ $\left(\mathrm{CH}_{2}\right)$.

HRMS (EI+) m/z: [M] ${ }^{+}$Calcd for $\mathrm{C}_{17} \mathrm{H}_{16}$ 220.1252; Found 220.1243 .

5. Substrate scope of boronic acids with methylenecyclohexane 7:<smiles>COc1ccc(CC2=CCCCC2)cc1</smiles>

$15 \mathbf{a}$

1-(4-methoxybenzyl)cyclohexene (15a): The compound 15a was prepared according to the general procedure. Purification by flash chromatography in PE/EA (99:1, v/v) afforded 15a as colorless oil (61 mg, 60\% yield). Exhibited spectra data identical to previous reports. ${ }^{17}$ 
IR (ATR) $v_{\max }: 2997,2923,2834,1610,1510,1440,1243,1175,1036,821 \mathrm{~cm}^{-1}$.

${ }^{1} \mathrm{H} \mathrm{NMR}\left(300 \mathrm{MHz}, \mathrm{CDCl}_{3}\right): \delta 7.11(\mathrm{~d}, J=8.4 \mathrm{~Hz}, 2 \mathrm{H}), 6.85(\mathrm{~d}, J=8.4 \mathrm{~Hz}, 2 \mathrm{H}), 5.47-5.46(\mathrm{~m}$, $1 \mathrm{H}), 3.81(\mathrm{~s}, 3 \mathrm{H}), 3.20(\mathrm{~s}, 2 \mathrm{H}), 2.07-2.01(\mathrm{~m}, 2 \mathrm{H}), 1.90-1.85(\mathrm{~m}, 2 \mathrm{H}), 1.61-1.56(\mathrm{~m}, 4 \mathrm{H})$.

${ }^{13} \mathrm{C} \mathrm{NMR}\left(75 \mathrm{MHz}, \mathrm{CDCl}_{3}\right): \delta 157.9(\mathrm{C}), 137.7(\mathrm{C}), 132.6(\mathrm{C}), 129.9(2 \times \mathrm{CH}), 122.7(\mathrm{CH}), 113.6$ (2xCH), $55.3\left(\mathrm{CH}_{3}\right), 43.9\left(\mathrm{CH}_{2}\right), 28.1\left(\mathrm{CH}_{2}\right), 25.4\left(\mathrm{CH}_{2}\right), 23.1\left(\mathrm{CH}_{2}\right), 22.6\left(\mathrm{CH}_{2}\right)$.

HRMS (EI $\left.{ }^{+}\right) \mathrm{m} / \mathrm{z}:[\mathrm{M}]^{+}$Calcd for $\mathrm{C}_{14} \mathrm{H}_{18} \mathrm{O}$ 202.1357; Found 202.1362.

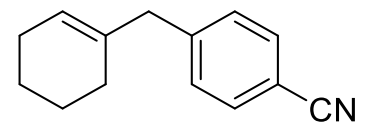

151

1-(4-cyanobenzyl)cyclohexene (15l): The compound 151 was prepared according to the general procedure. Purification by flash chromatography in PE/EA (99:1, v/v) afforded $\mathbf{1 5 l}$ as clear oil (52 mg, $53 \%$ yield).

IR (ATR) v $v_{\max }$ : 2924, 2856, 2835, 2227, 1666, 1606, 1503, 1437, 1414, 1175, 1110, 1020 , $948,919,853,821 \mathrm{~cm}^{-1}$.

${ }^{1} \mathrm{H}$ NMR $\left(300 \mathrm{MHz}, \mathrm{CDCl}_{3}\right): \delta$ 7.60-7.53 (m, 2H), 7.29-7.26 (m, 2H), 5.49-5.46 (m, 1H), 3.28 (brs, 2H), 2.07-1.98 (m, 2H), 1.85-1.77 (m, 2H), 1.62-1.50 (m, 4H).

${ }^{13} \mathrm{C}$ NMR (75 MHz, $\mathrm{CDCl}_{3}$ ): $\delta 146.7$ (C), $135.9(\mathrm{C}), 132.1$ (2xCH), 129.7 (2xCH), 124.5

$(\mathrm{CH}), 119.3(\mathrm{C}), 109.8(\mathrm{C}), 44.8\left(\mathrm{CH}_{2}\right), 28.2\left(\mathrm{CH}_{2}\right), 25.4\left(\mathrm{CH}_{2}\right), 22.9\left(\mathrm{CH}_{2}\right), 22.3\left(\mathrm{CH}_{2}\right)$.

HRMS (EI $\left.{ }^{+}\right) \mathrm{m} / \mathrm{z}$ : [M] $]^{+}$Calcd for $\mathrm{C}_{14} \mathrm{H}_{15} \mathrm{~N}$ 197.1204; Found 197.1209.

6. Substrate scope of boronic acids with allylbenzene derivatives 8-9:

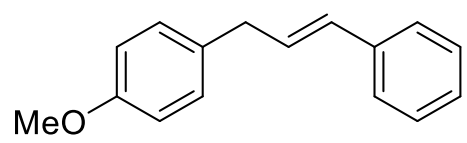

$16 \mathbf{a}$

(E) 3-(4-Methoxyphenyl)-1-phenyl-propene (16a): The compound 15a was prepared according to the general procedure from 3-phenylpropene 8. Purification by flash chromatography in PE/EA (98:2, v/v) afforded 16a as clear oil (57 mg, 51\% yield). Exhibited spectra data identical to previous reports. ${ }^{14}$

IR (ATR) $v_{\max }: 2971,2902,2836,1723,1683,1609,1510,1462,1301,1245,1175,1033,967$, $828,753,697 \mathrm{~cm}^{-1}$. 
${ }^{1} \mathrm{H}$ NMR (300 MHz, $\left.\mathrm{CDCl}_{3}\right): \delta$ 7.38-7.16 (m, 7H), 6.90-6.85 (m, 2H), 6.48-6.30 (m, 2H), 3.81 $(\mathrm{s}, 3 \mathrm{H}), 3.51(\mathrm{~d}, J=6.2 \mathrm{~Hz}, 2 \mathrm{H})$.

${ }^{13} \mathrm{C} \mathrm{NMR}\left(75 \mathrm{MHz}, \mathrm{CDCl}_{3}\right): \delta 158.0(\mathrm{C}), 137.9(\mathrm{C}), 132.1(\mathrm{C}), 130.7(\mathrm{CH}), 129.6(\mathrm{CH}), 129.5$ $(2 \mathrm{xCH}), 128.5(2 \mathrm{xCH}), 127.0(\mathrm{CH}), 126.1(2 \mathrm{xCH}), 113.9(2 \mathrm{xCH}), 55.3\left(\mathrm{CH}_{3}\right), 38.4\left(\mathrm{CH}_{2}\right)$.

HRMS (EI $\left.{ }^{+}\right) \mathrm{m} / \mathrm{z}$ : [M] ${ }^{+}$Calcd for $\mathrm{C}_{16} \mathrm{H}_{16} \mathrm{O} 224.1201$; Found 224.1197.

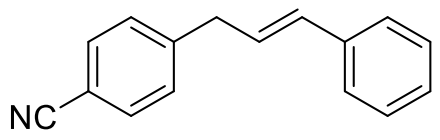

161

(E) 3-(4-Cyanophenyl)-1-phenyl-propene (16l): The compound 161 was prepared according to the general procedure from 3-phenylpropene 8. Purification by flash chromatography in PE/EA (98:2, v/v) afforded $16 \mathbf{l}$ as clear oil (59 mg, 54\% yield). Exhibited spectra data identical to previous reports. ${ }^{15}$

IR (ATR) $v_{\max }: 3027,2227,1703,1605,1495,1449$, 1414, 1176, 1111, 1020, 966, 927, 829, $751,696 \mathrm{~cm}^{-1}$.

${ }^{1} \mathrm{H}$ NMR (300 MHz, $\left.\mathrm{CDCl}_{3}\right): \delta$ 7.56-7.52 (m, 2H), 7.33-7.15 (m, 7H), 6.45-6.40 (m, 1H), 6.24 (dt, $J=15.8,6.8 \mathrm{~Hz}, 1 \mathrm{H}), 3.55(\mathrm{~d}, J=6.7 \mathrm{~Hz}, 2 \mathrm{H})$.

${ }^{13} \mathrm{C} \mathrm{NMR}\left(75 \mathrm{MHz}, \mathrm{CDCl}_{3}\right): \delta 145.9(\mathrm{C}), 136.9(\mathrm{C}), 132.5(\mathrm{CH}), 132.4(2 \mathrm{xCH}), 129.5(2 \mathrm{xCH})$, $128.7(2 \times C H), 127.6(\mathrm{CH}), 127.2(\mathrm{CH}), 126.3(2 \times C H), 119.1(\mathrm{C}), 110.2(\mathrm{C}), 39.4\left(\mathrm{CH}_{2}\right)$.

HRMS (EI $\left.{ }^{+}\right) \mathrm{m} / \mathrm{z}:[\mathrm{M}]^{+}$Calcd for $\mathrm{C}_{16} \mathrm{H}_{13} \mathrm{~N} 219.1048$; Found 219.1047.

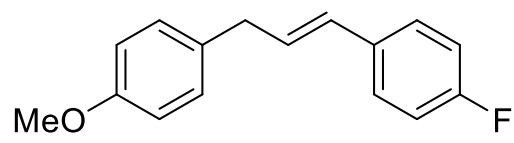

$17 \mathbf{a}$

(E) 1-(4-Fluorophenyl)-3-(4-methoxyphenyl)propene (17a): The compound 17a was prepared according to the general procedure from 3-(4-fluorophenyl)propene 9. Purification by flash chromatography in PE/EA (98:2, v/v) afforded 17a as clear oil (58 mg, $48 \%$ yield). Exhibited spectra data identical to previous reports. ${ }^{16}$

IR (ATR) $v_{\max }: 3003,2907,2835,1601,1506,1463,1300,1224,1175,1157,1033,966,825$ $\mathrm{cm}^{-1}$.

${ }^{1} \mathrm{H}$ NMR (300 MHz, $\left.\mathrm{CDCl}_{3}\right): \delta$ 7.36-7.30 (m, 2H), 7.20-7.16 (m, 2H), 7.03-6.95 (m, 2H), 6.91-6.86 (m, 2H), 6.43-6.38 (m, 1H), 6.27 (dt, $J=15.7,6.6 \mathrm{~Hz}, 1 \mathrm{H}), 3.81$ (s, 3H), 3.49 (d, $J$ $=6.5 \mathrm{~Hz}, 2 \mathrm{H})$. 
${ }^{13} \mathrm{C}$ NMR $\left(75 \mathrm{MHz}, \mathrm{CDCl}_{3}\right): \delta 162.0\left(\mathrm{C}-\mathrm{F},{ }^{1} J_{\mathrm{C}-\mathrm{F}}=245.9 \mathrm{~Hz}\right), 158.2(\mathrm{C}), 133.8\left(\mathrm{C}-\mathrm{F},{ }^{4} J_{\mathrm{C}-\mathrm{F}}=\right.$ $3.3 \mathrm{~Hz}), 132.2(\mathrm{C}), 129.7(2 \mathrm{xCH}), 129.6(2 \mathrm{xCH}), 129.5\left(\mathrm{CH}-\mathrm{F},{ }^{5} J_{\mathrm{C}-\mathrm{F}}=2,2 \mathrm{~Hz}\right), 127.6(2 \mathrm{xCH}-$ $\left.\mathrm{F},{ }^{3} J_{\mathrm{C}-\mathrm{F}}=7.9 \mathrm{~Hz}\right), 115.5\left(2 \mathrm{xCH}-\mathrm{F},{ }^{2} J_{\mathrm{C}-\mathrm{F}}=21.5 \mathrm{~Hz}\right), 114.0(\mathrm{CH}), 55.4\left(\mathrm{CH}_{3}\right), 38.5\left(\mathrm{CH}_{2}\right)$. ${ }^{19} \mathrm{~F}$ NMR $\left(282 \mathrm{MHz}, \mathrm{CDCl}_{3}\right) \delta-115.3--115.4(\mathrm{~m})$.

HRMS (EI ${ }^{+}$m/z: [M] $]^{+}$Calcd for $\mathrm{C}_{16} \mathrm{H}_{15} \mathrm{FO}$ 242.1106; Found 242.1101.<smiles>N#Cc1ccc(C/C=C/c2ccc(F)cc2)cc1</smiles>

171

(E) 3-(4-Cyanophenyl)-1-(4-fluorophenyl)propene (17l) The compound 171 was prepared according to the general procedure from 3-(4-fluorophenyl)propene 9. Purification by flash chromatography in PE/EA (98:2, v/v) afforded $\mathbf{1 7 l}$ as clear oil (63 $\mathrm{mg}, 53 \%$ yield).

IR (ATR) $v_{\max }: 3038,2227,1705,1602,1506,1413,1297,1224,1157,1095,967,819,767$ $\mathrm{cm}^{-1}$.

${ }^{1} \mathrm{H}$ NMR (300 MHz, $\left.\mathrm{CDCl}_{3}\right): \delta$ 7.62-7.59 (m, 2H), 7.36-7.29 (m, 4H), 7.03-6.96 (m, 2H), 6.46-6.40 (m, 1H), $6.21(\mathrm{dt}, J=15.8,6.9 \mathrm{~Hz}, 1 \mathrm{H}), 3.59$ (d, $J=6.8 \mathrm{~Hz}, 2 \mathrm{H})$.

${ }^{13} \mathrm{C} \mathrm{NMR}\left(75 \mathrm{MHz}, \mathrm{CDCl}_{3}\right): \delta 162.5\left(\mathrm{C}-\mathrm{F},{ }^{1} J_{\mathrm{C}-\mathrm{F}}=246.7 \mathrm{~Hz}\right), 145.8(\mathrm{C}), 133.2\left(\mathrm{C}-\mathrm{F},{ }^{4} J_{\mathrm{C}-\mathrm{F}}=\right.$ $3.2 \mathrm{~Hz}), 132.5(2 \mathrm{xCH}), 131.3(\mathrm{CH}), 129.5(2 \mathrm{xCH}), 127.8\left(2 \mathrm{xCH}-\mathrm{F},{ }^{3} J_{\mathrm{C}-\mathrm{F}}=8.0 \mathrm{~Hz}\right), 127.0$ $\left(\mathrm{CH}-\mathrm{F},{ }^{5} J_{\mathrm{C}-\mathrm{F}}=2.2 \mathrm{~Hz}\right), 119.1(\mathrm{C}), 115.6\left(2 \mathrm{xCH}-\mathrm{F},{ }^{2} J_{\mathrm{C}-\mathrm{F}}=21.6 \mathrm{~Hz}\right), 110.03(\mathrm{C}), 39.4\left(\mathrm{CH}_{2}\right)$. ${ }^{19} \mathrm{~F} \mathrm{NMR}\left(282 \mathrm{MHz}, \mathrm{CDCl}_{3}\right) \delta-114.4-114.5(\mathrm{~m})$.

HRMS (EI ${ }^{+}$m/z: [M] ${ }^{+}$Calcd for $\mathrm{C}_{16} \mathrm{H}_{12} \mathrm{FN} 237.0953$; Found 237.0955.

7. Allylic C-H arylation with 2-methyl-1-phenylpropene 10:

Scheme 2: Allylic C-H arylation with 2-methyl-1-phenylpropene

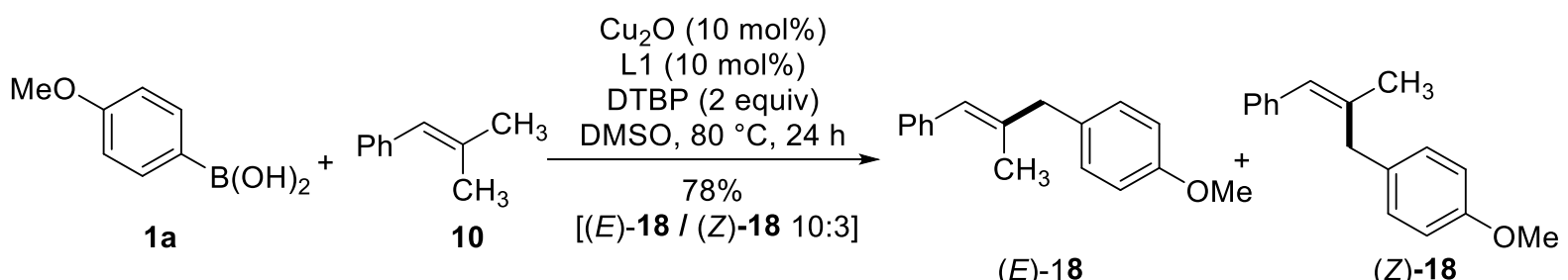

Isomers $(E)-\mathbf{1 8}$ and $(Z)-\mathbf{1 8}$ are known in the literature and well characterized, see references 16. Unfortunately, we are unable to separate these two isomers by flash chromatography. 

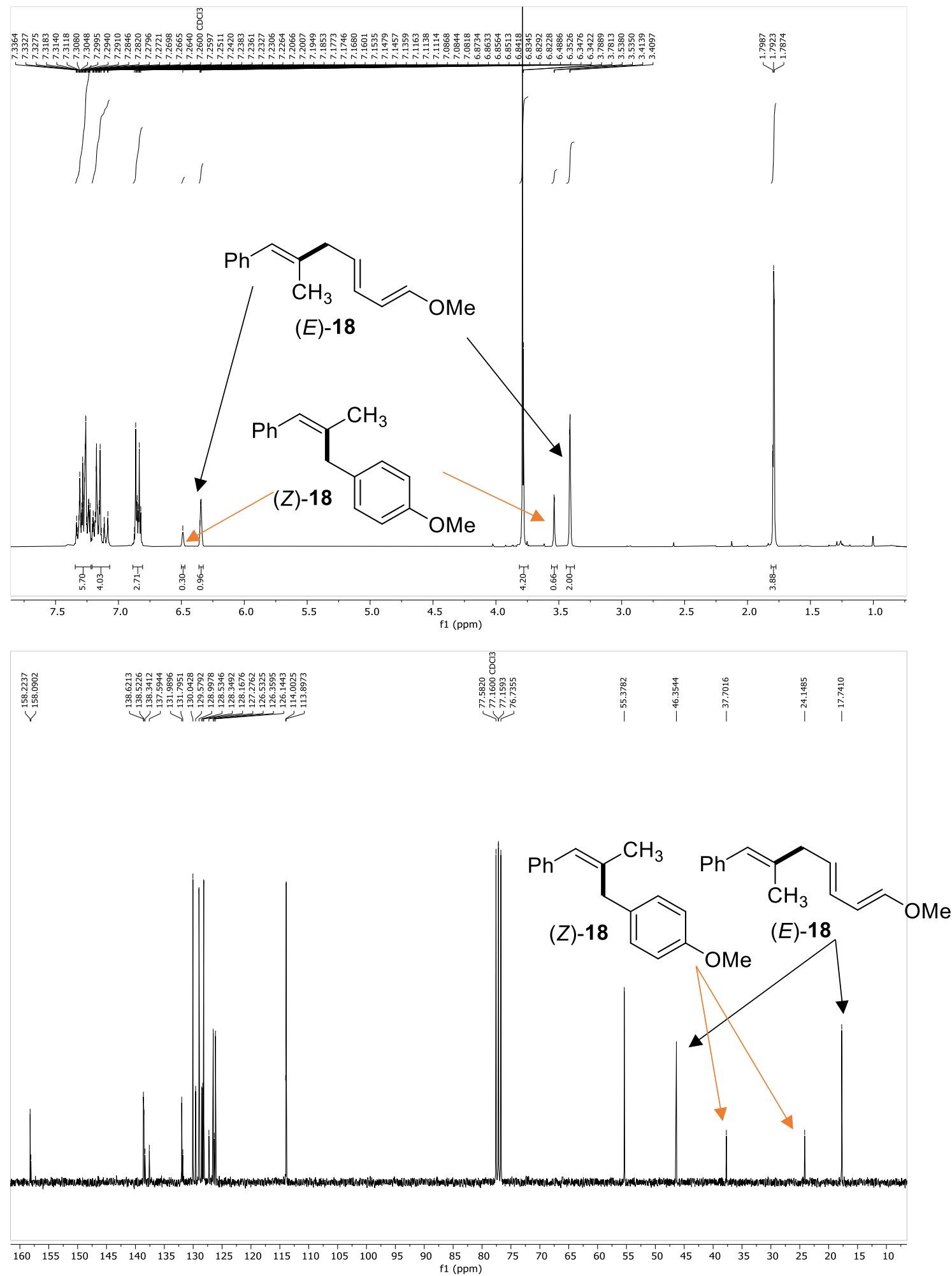

8. Allylic C-H arylation with 1-methylcyclohexene 11:

Scheme 3: Allylic C-H arylation with 1-methylcyclohexene 

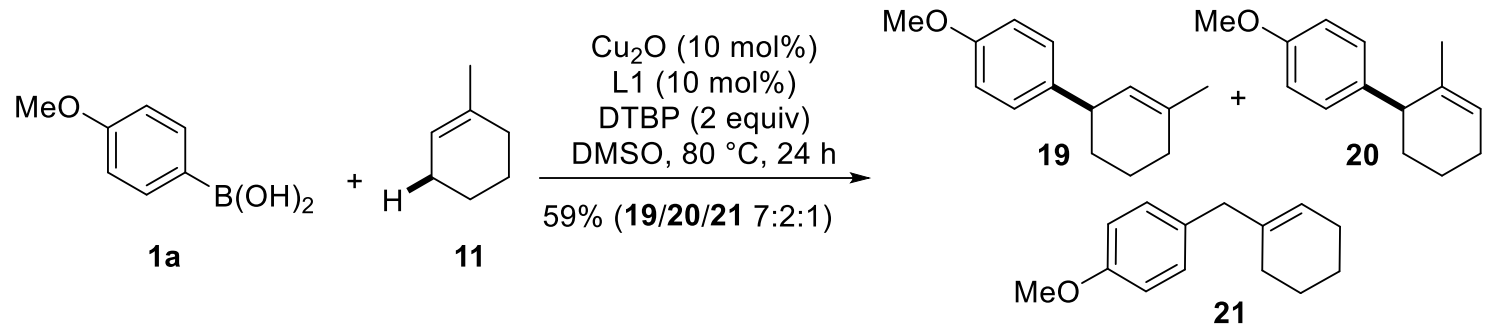

Products 19, 20 and 21 are known in the literature and well characterized, see references 17-19. Unfortunately, we are unable to separate these three isomers by flash chromatography.

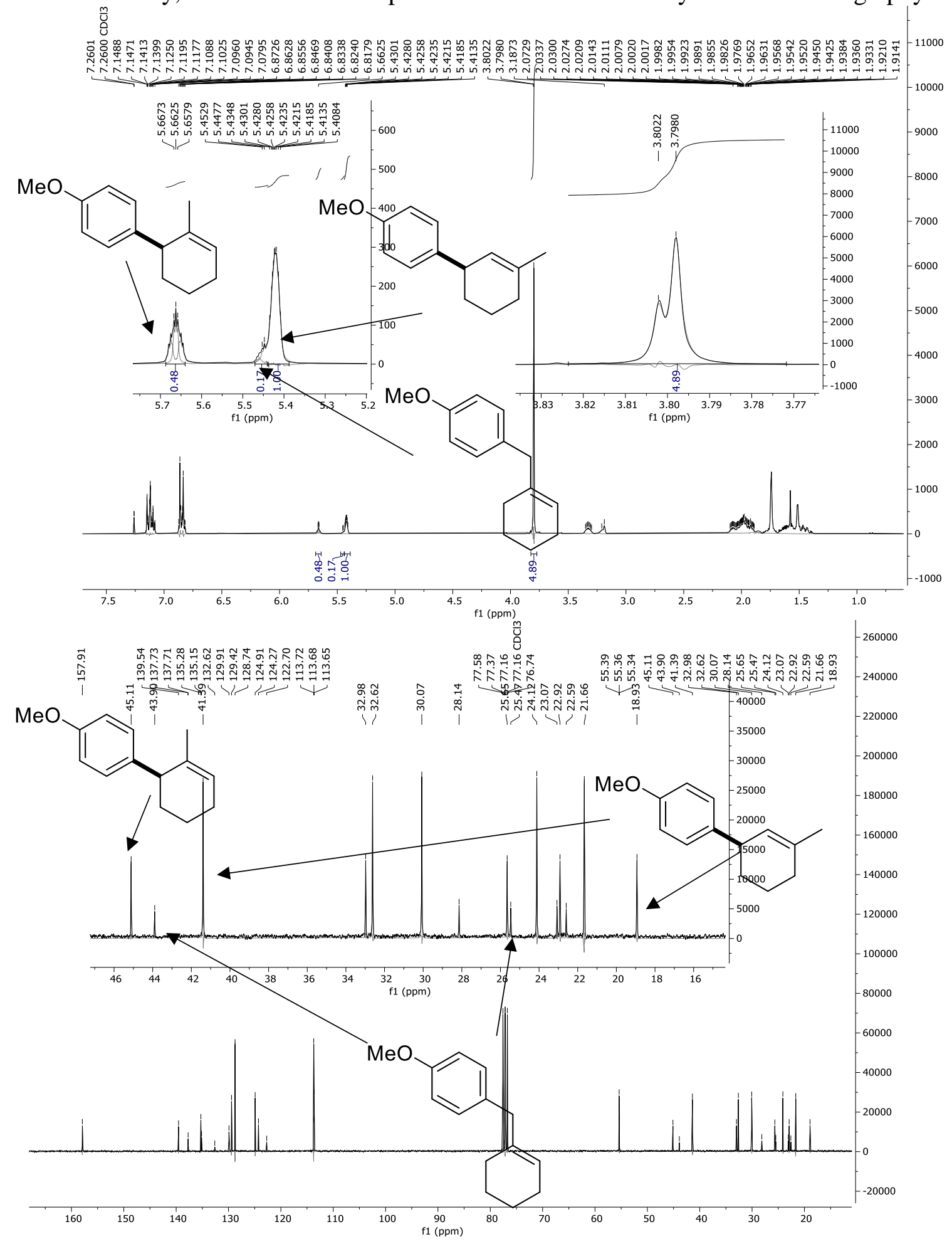


9. Radical trapping experiment using TEMPO:

Scheme 4: Radical-trapping experiment using TEMPO

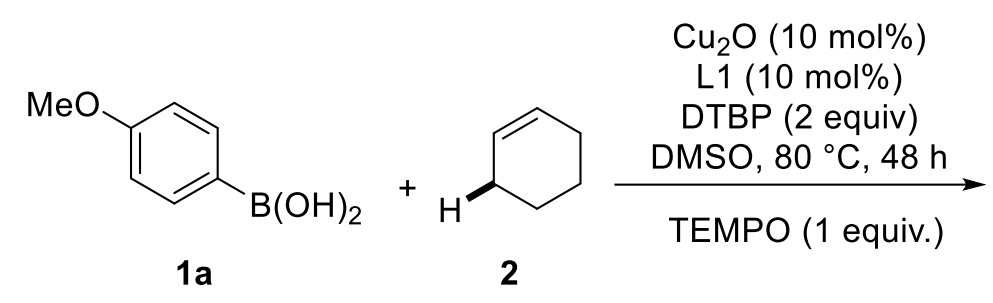

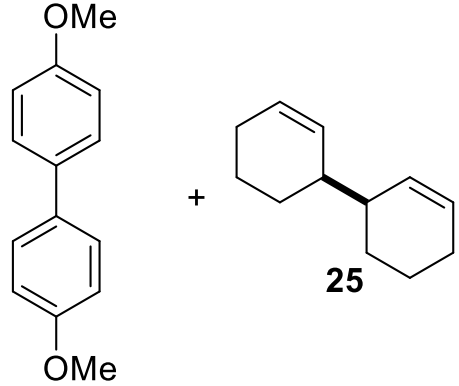

24

4-Methophenylboronic acid (0.5 mmol, 1 equiv.), $\mathrm{Cu}_{2} \mathrm{O}$ (0.05 mmol, $\left.10 \mathrm{~mol} \%\right)$, L1 (0.05 mmol, $10 \mathrm{~mol} \%$ ) and TEMPO (1 equiv.) were placed in a sealed tube which was then evacuated and backfilled three times with nitrogen. DMSO (1.5 mL), cyclohexene (5 mmol, 10 equiv.) and DTBP ( $1 \mathrm{mmol}, 2$ equiv.) were added and the mixture was allowed to heat at $80^{\circ} \mathrm{C}$ for 48 h. The reaction was then heated to $80{ }^{\circ} \mathrm{C}$. After stirring for $48 \mathrm{~h}$, the reaction was cooled down to room temperature and no product 3a was observed by GC-MS. 1 equivalent of TEMPO was sufficient to inhibit the reaction completely.

Figure 1: GC-MS spectra for the radical trapping using 1 equivalent of TEMPO

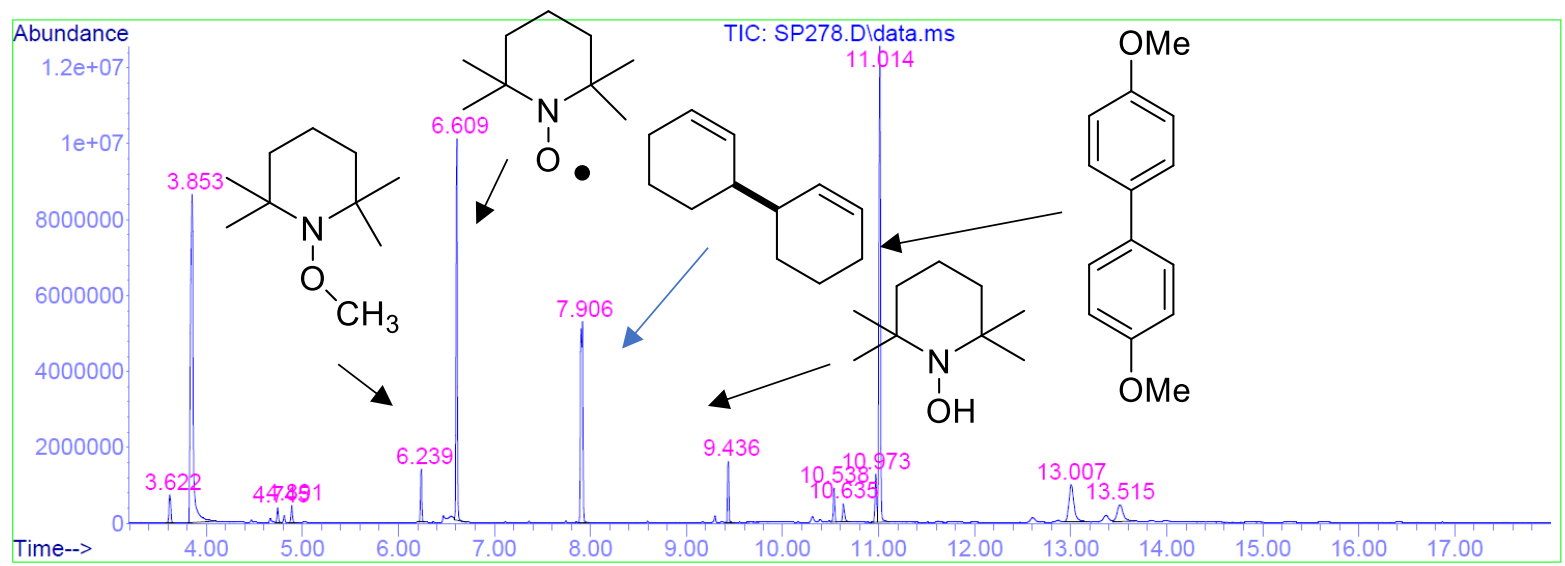



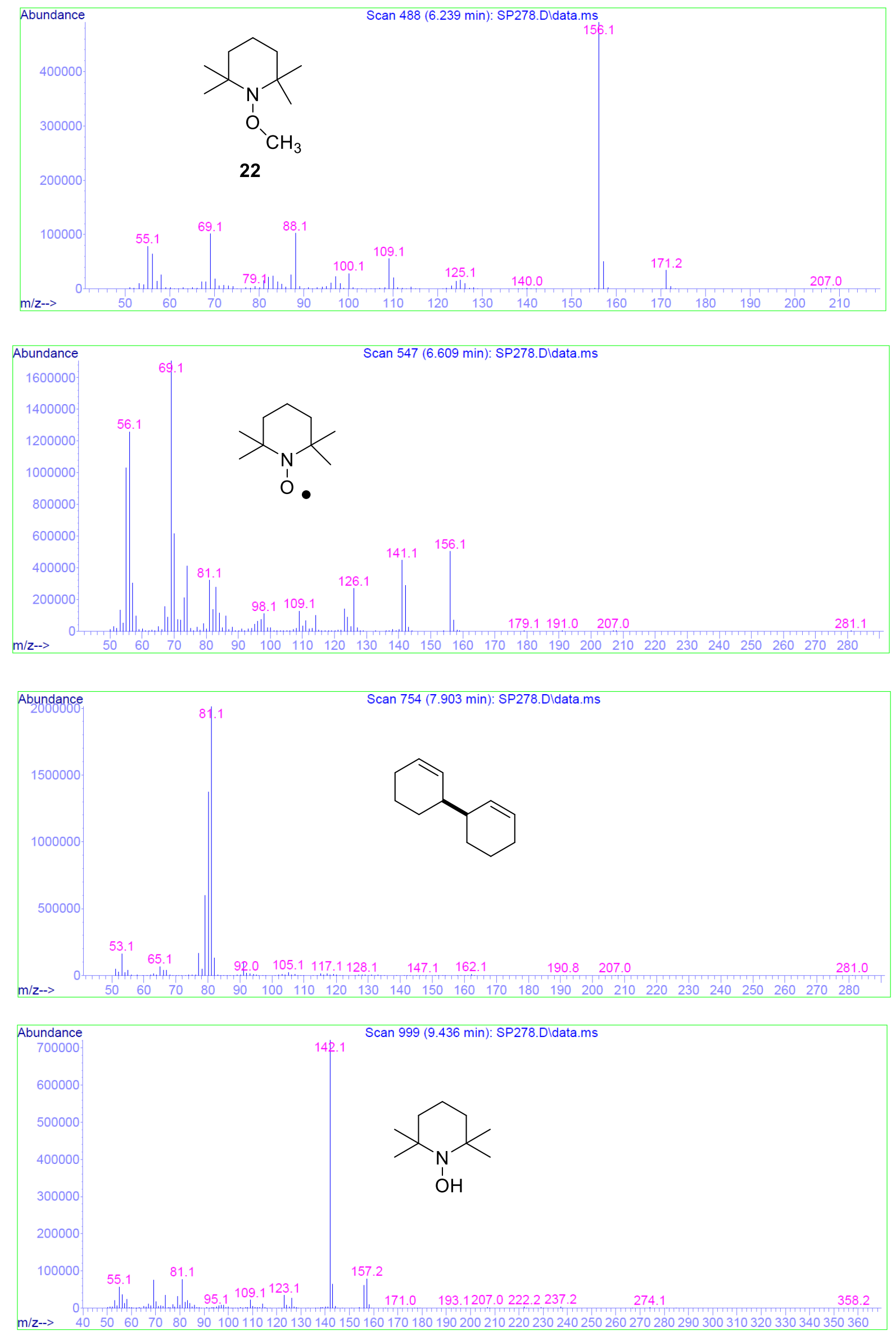


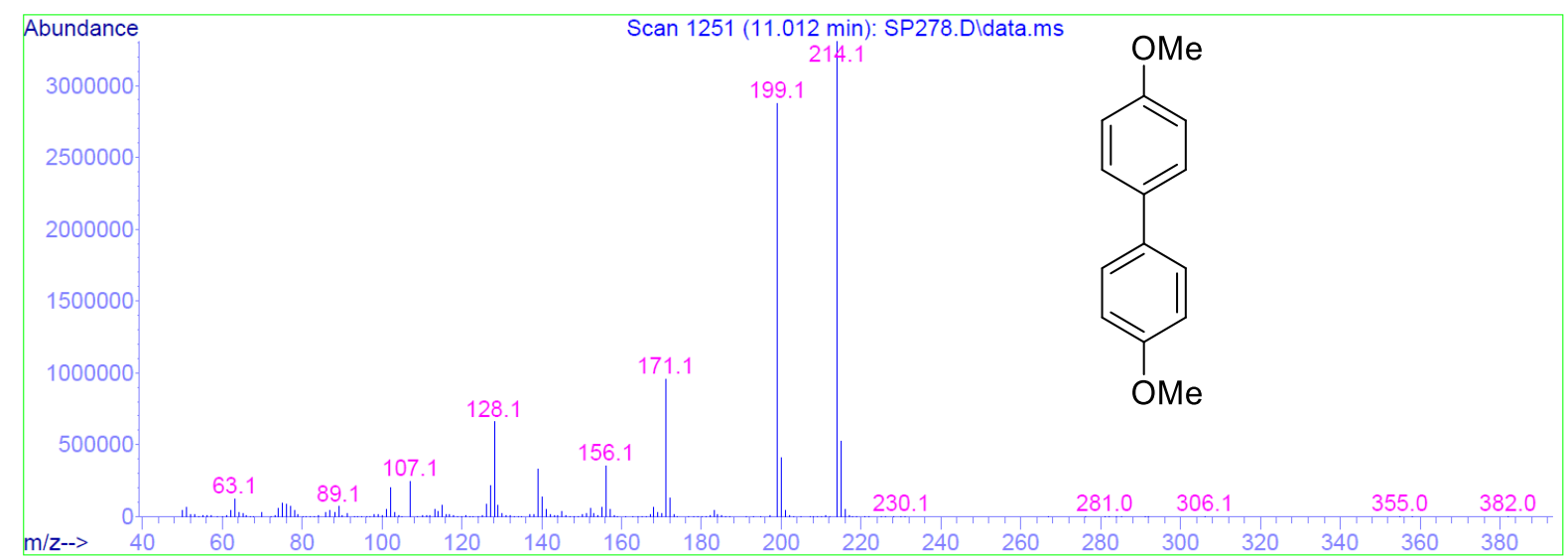

\section{References}

1) Sekine, M.; Ilies, L.; Nakamura, E. Org. Lett. 2013, 15, 714-717.

2) Baker, L.; Minehan, T. J. Org. Chem. 2004, 69, 3957-3960.

3) Gonza' lez, J.; Scha' fer, P.; Fletcher, S. P. Organometallics 2019, 38, 3991-3995.

4) Correia, R.; DeShong, P. J. Org. Chem. 2001, 66, 7159-7165.

5) Lee, K.; Lee, J.; Lee, P. H. J. Org. Chem. 2002, 67, 8265-8268

6) Papoian, V.; Minehan, T. J. Org. Chem. 2008, 73, 7376-7379.

7) Bouyssi, D.; Gerusz, V.; Balme, G. Eur. J. Org. Chem. 2002, 2445-2448.

8) Cuthbertson, J. D.; MacMillan, D. W. C. Nature 2015, 519, 74-77.

9) Vila, C.; Hornillos, V.; Fañanás-Mastrala, M.; Feringa, B. L. Org. Biomol. Chem. 2014, 12, 9321-9323.

10) Han, X.; Zhang, Y.; Wu, J. J. Am. Chem. Soc. 2010, 132, 4104-4106.

11) Guignard, R. F.; Petit, L.; Zard, S. Z. Org. Lett. 2013, 15, 4178-4181.

12) Kamigata, N.; Satoh, A.; Kondoh, T.; Kameyama, M. Bull. Chem. Soc. Jpn. 1988, 61, 35753580 .

13) Walker, B. R.; Sevov, C. R. ACS Catal. 2019, 9, 7197-7203.

14) Lv, L.; Zhu, D.; Li, C.-J. Nat. Commun 2019, 10, 715.

15) Anka-Lufford, L. L.; Prinsell, M. R.; Weix, D. J. J. Org. Chem. 2012, 77, 9989-10000.

16) Zhang, Y.; Yin, S.-C.; Lu, J.-M. Tetrahedron 2015, 71, 544-549.

17) Luo, H.; Hu, G.; Li, P. J. Org. Chem. 2019, 84, 10569-10578.

18) Malkov, A. V.; Davis, S. L.; Baxendale, I. R.; Mitchell, W. L.; Kocvsky, P. J. Org. Chem. 1999, 64, 2751-2764.

19) Baker, L.; Minehan, T. J. Org. Chem. 2004, 69, 3957-3960.

\section{${ }^{1}$ H NMR \& ${ }^{13}$ C NMR Spectra}




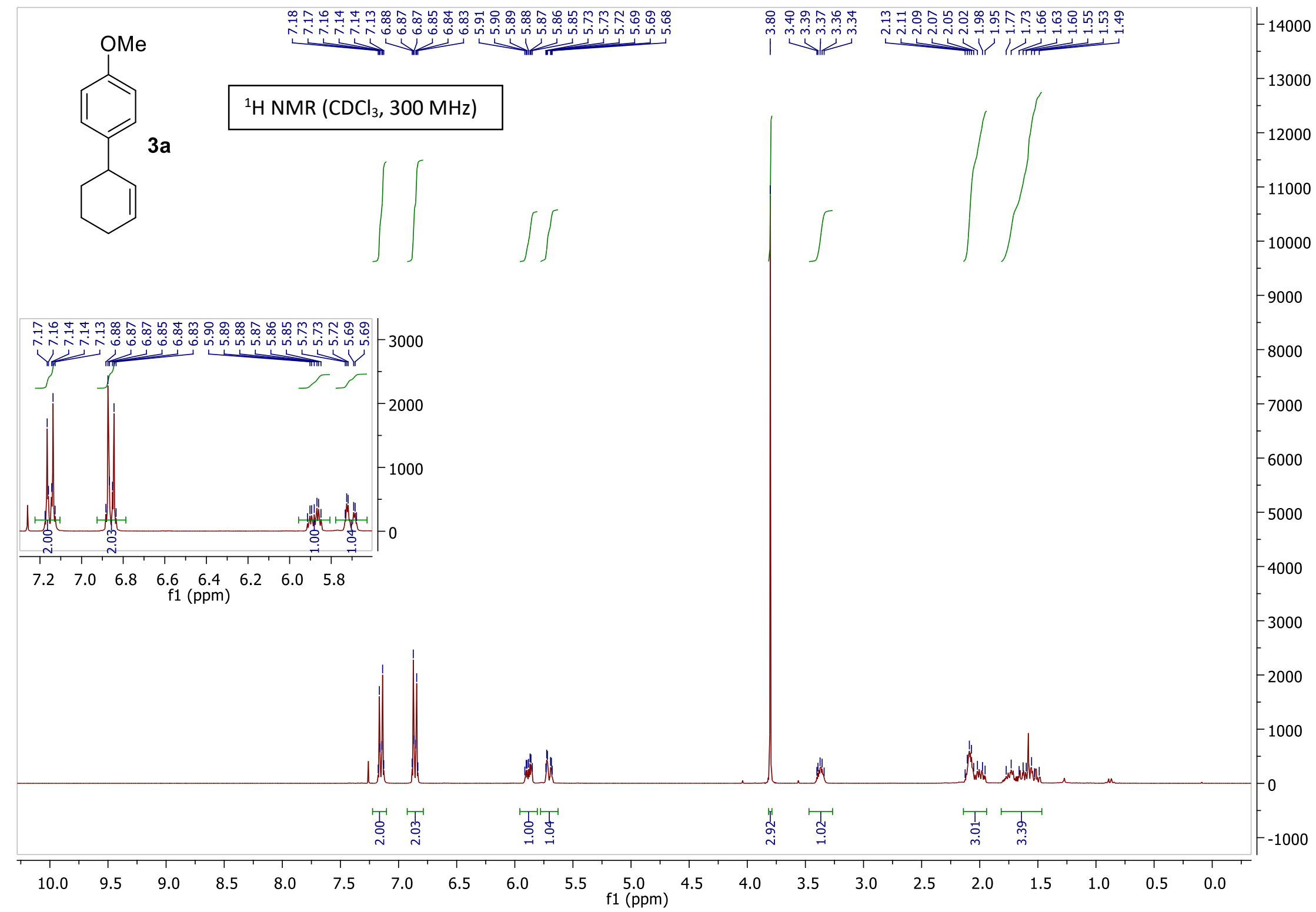




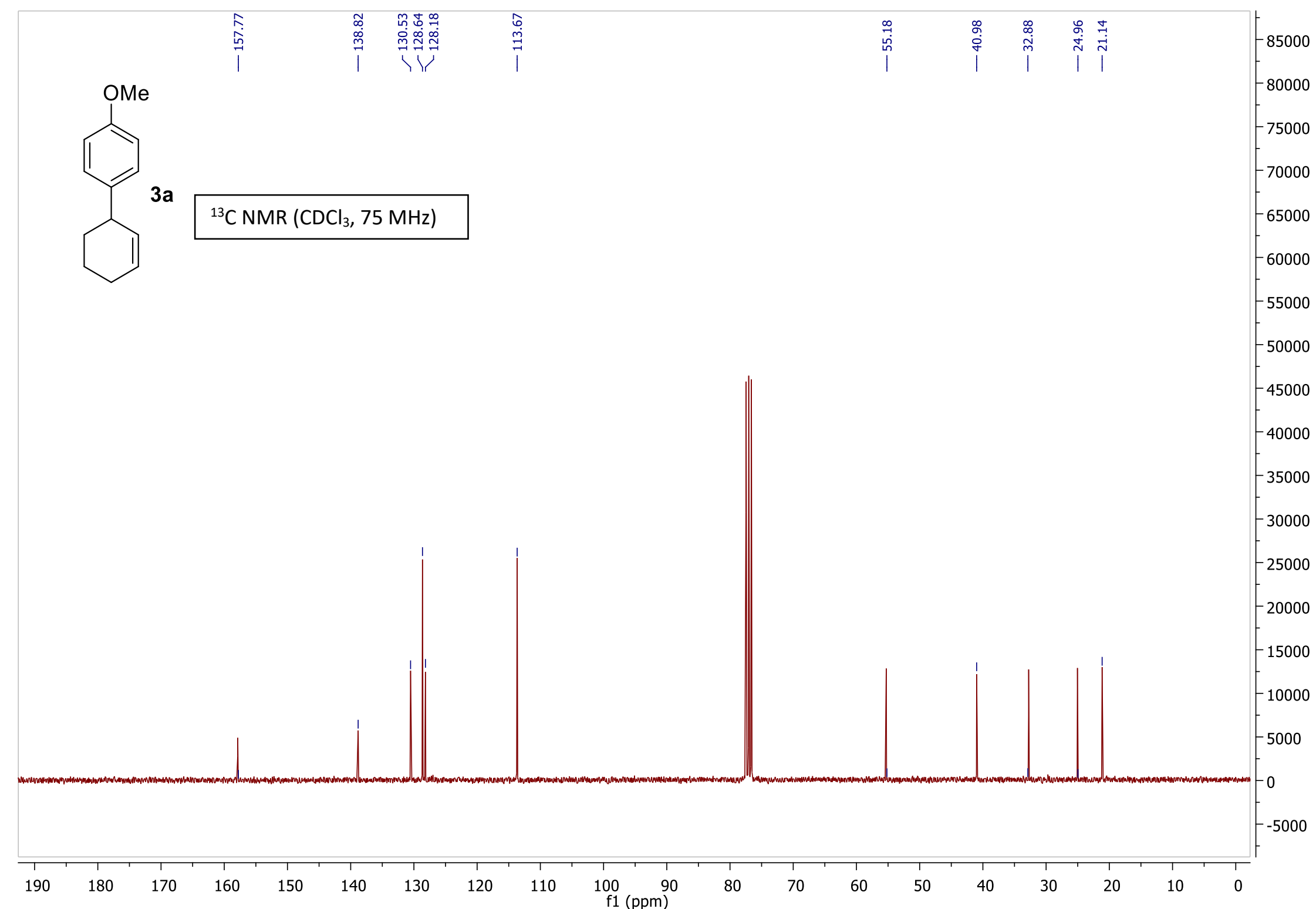




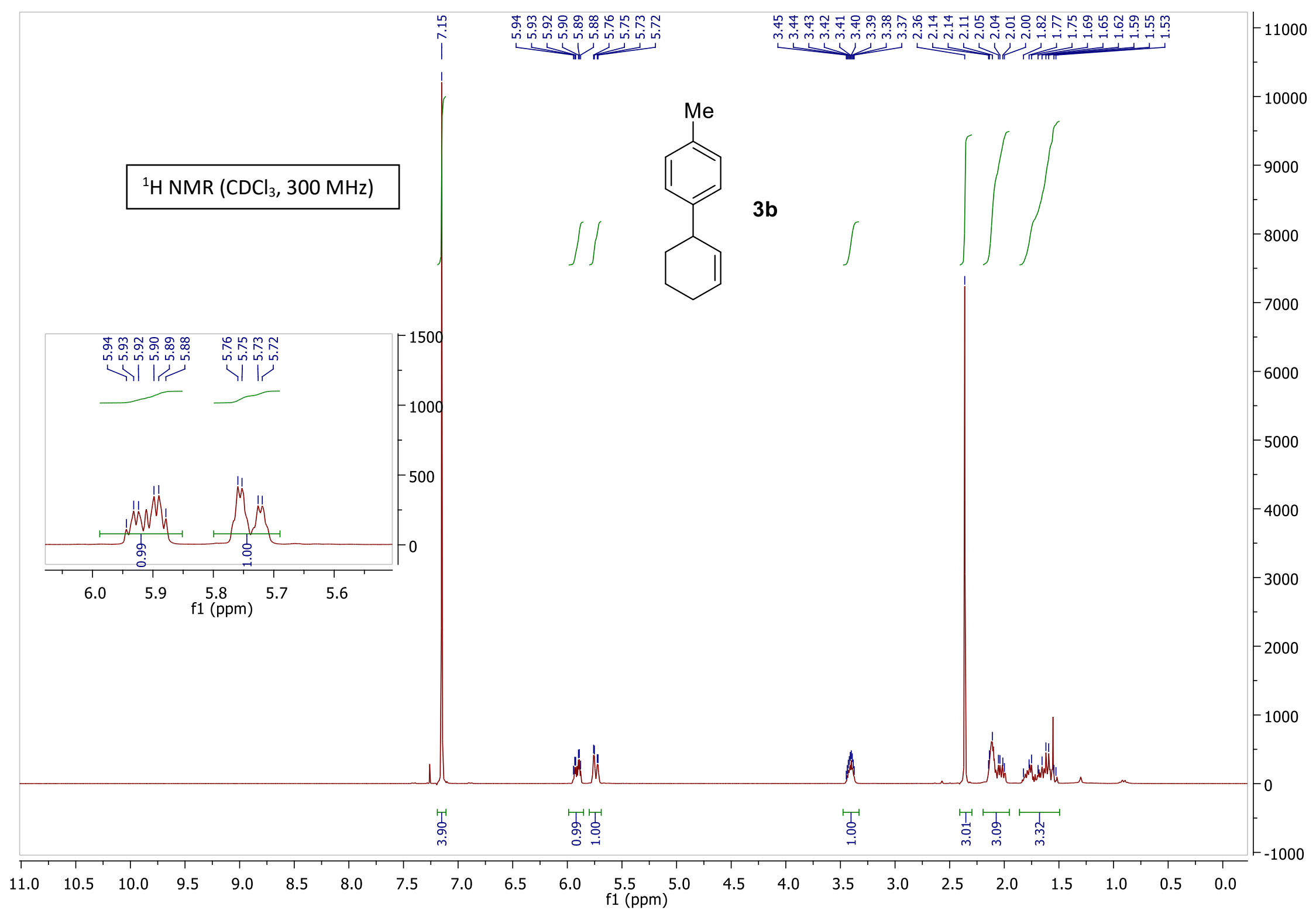




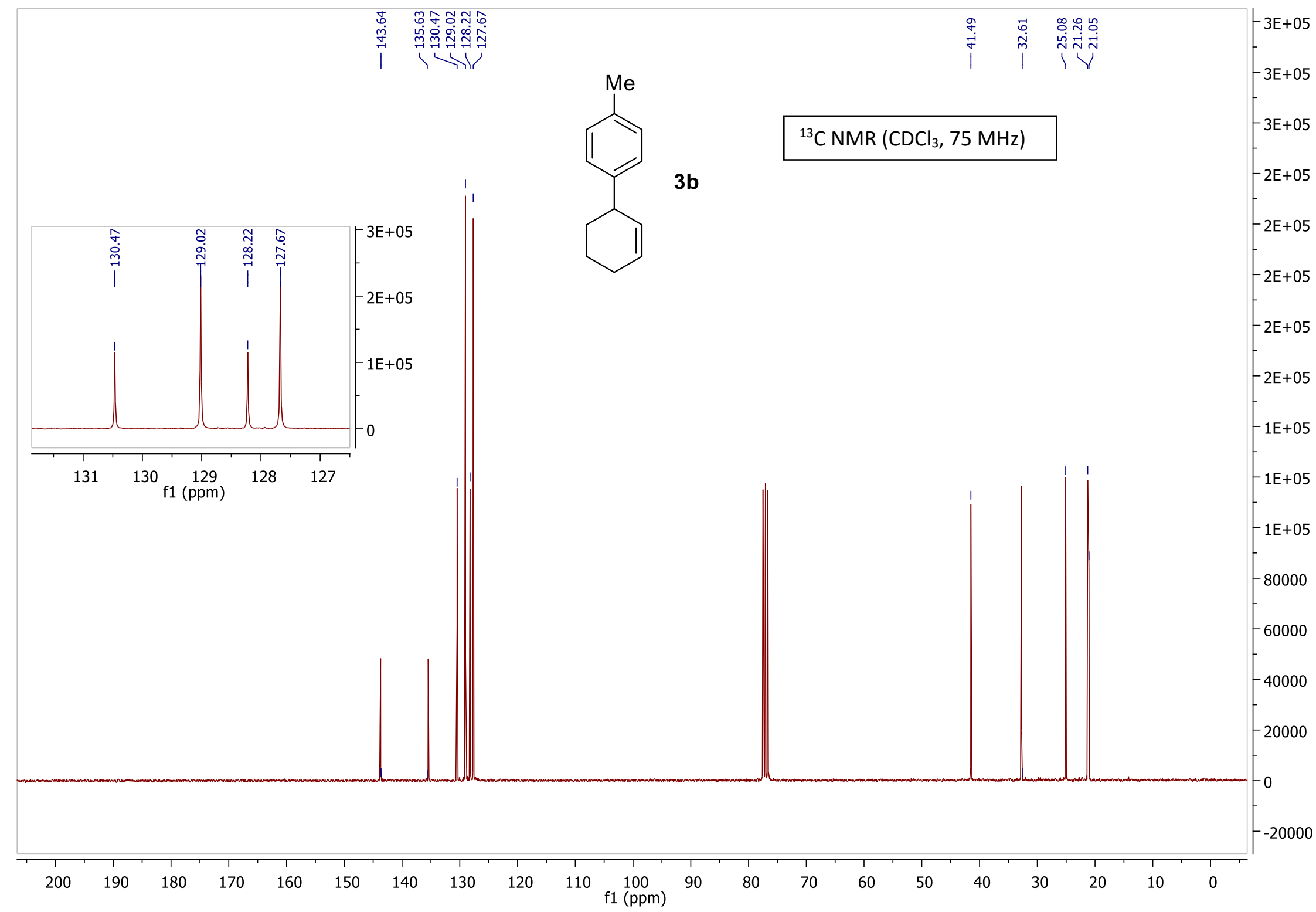




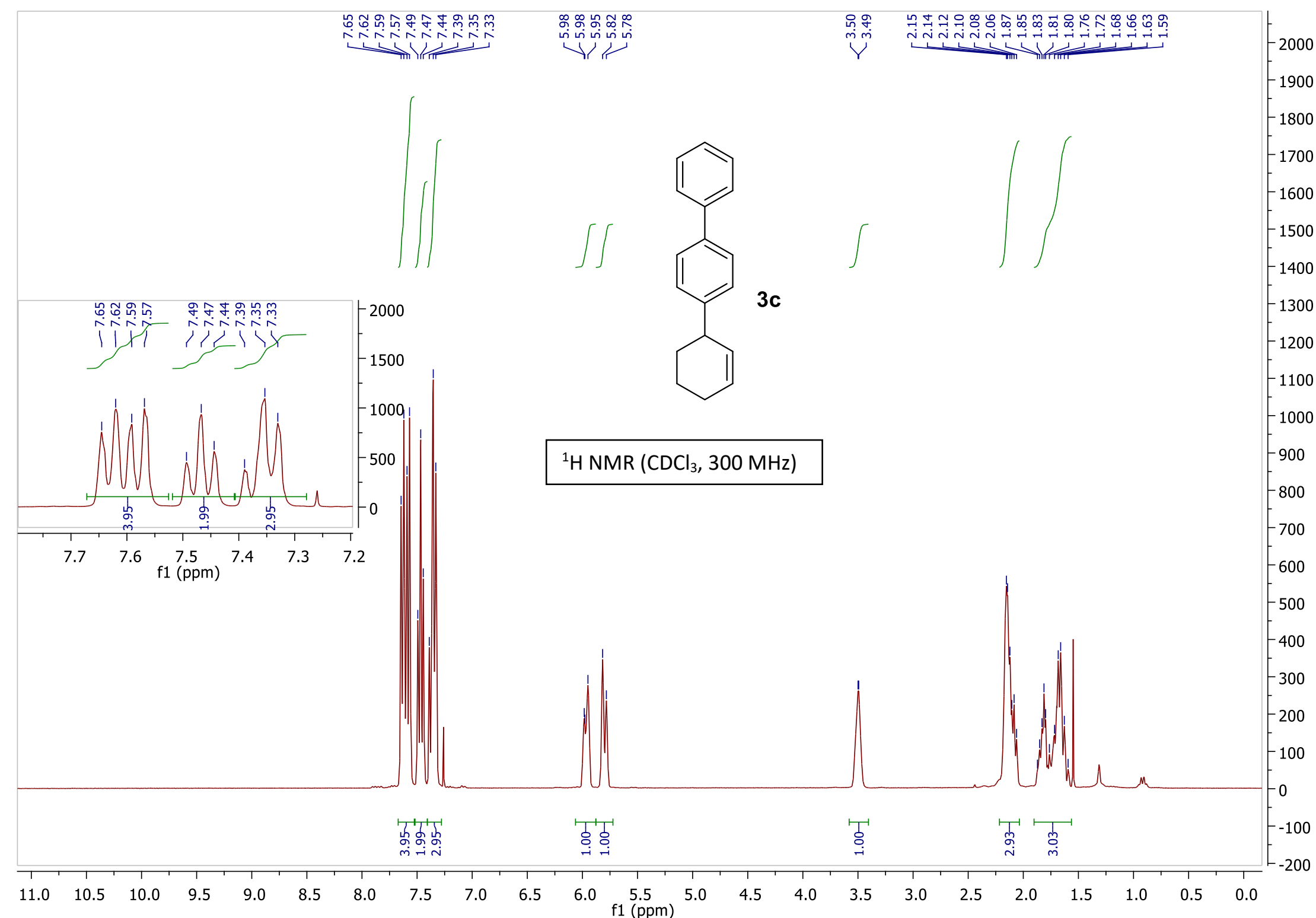




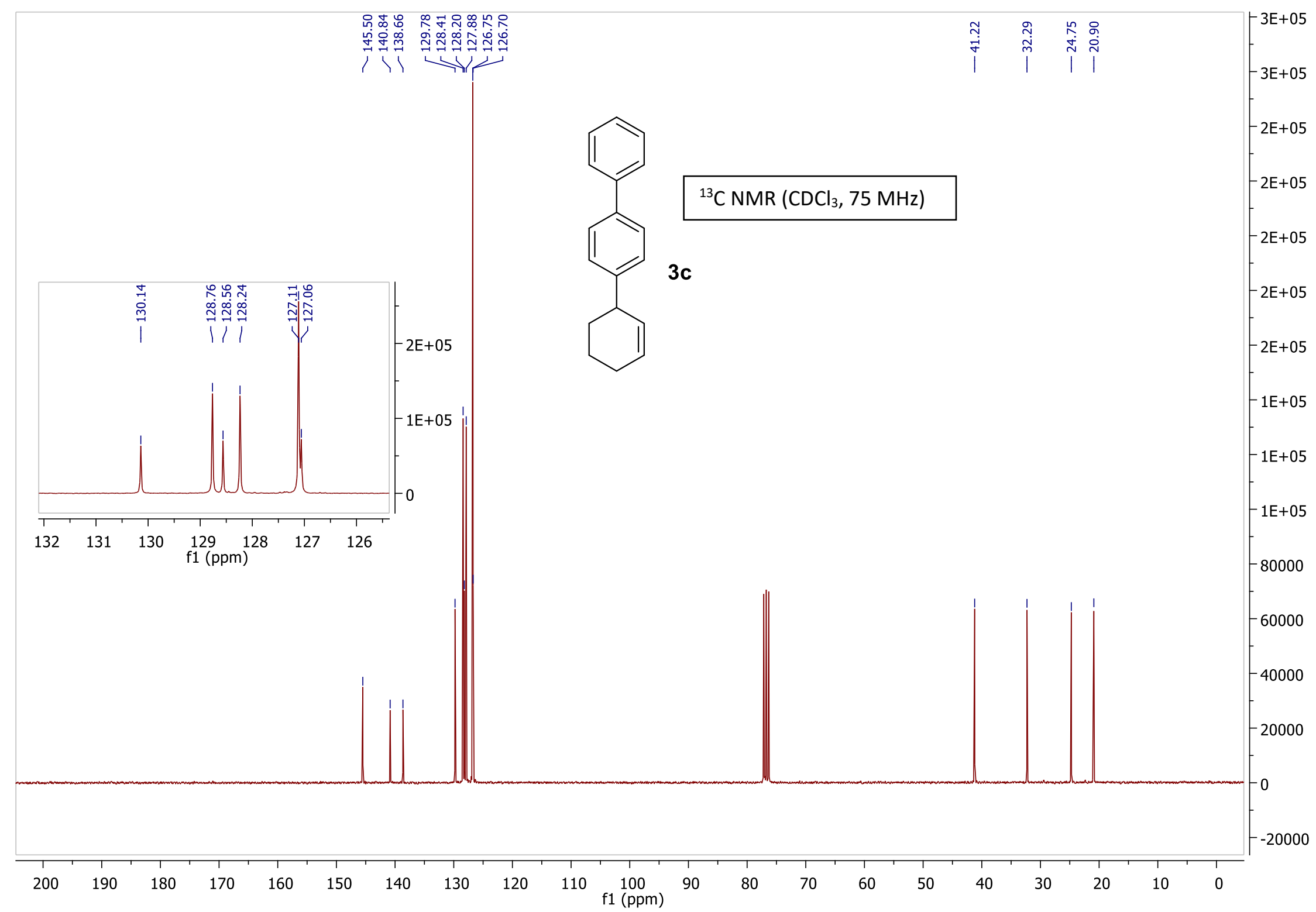




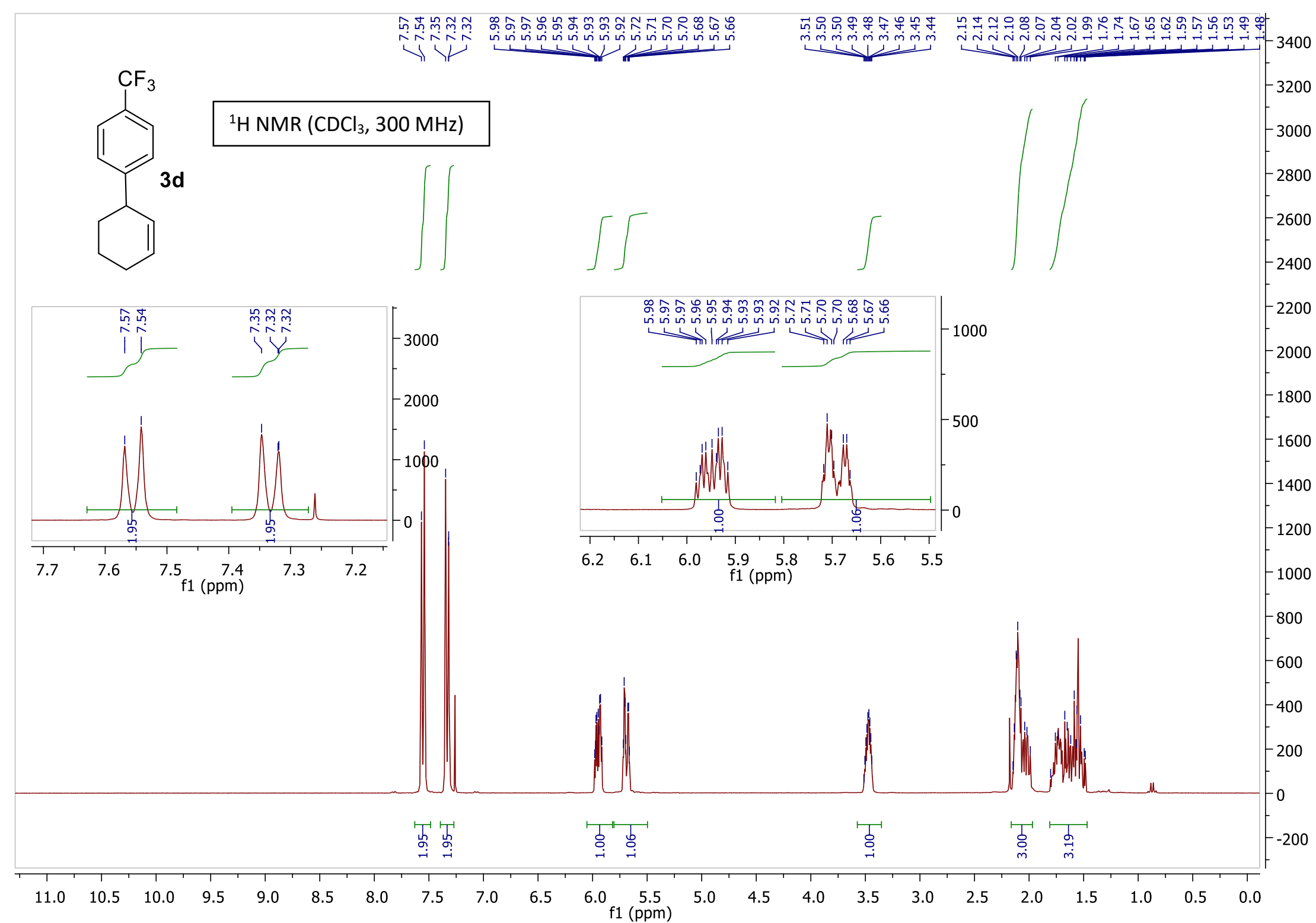




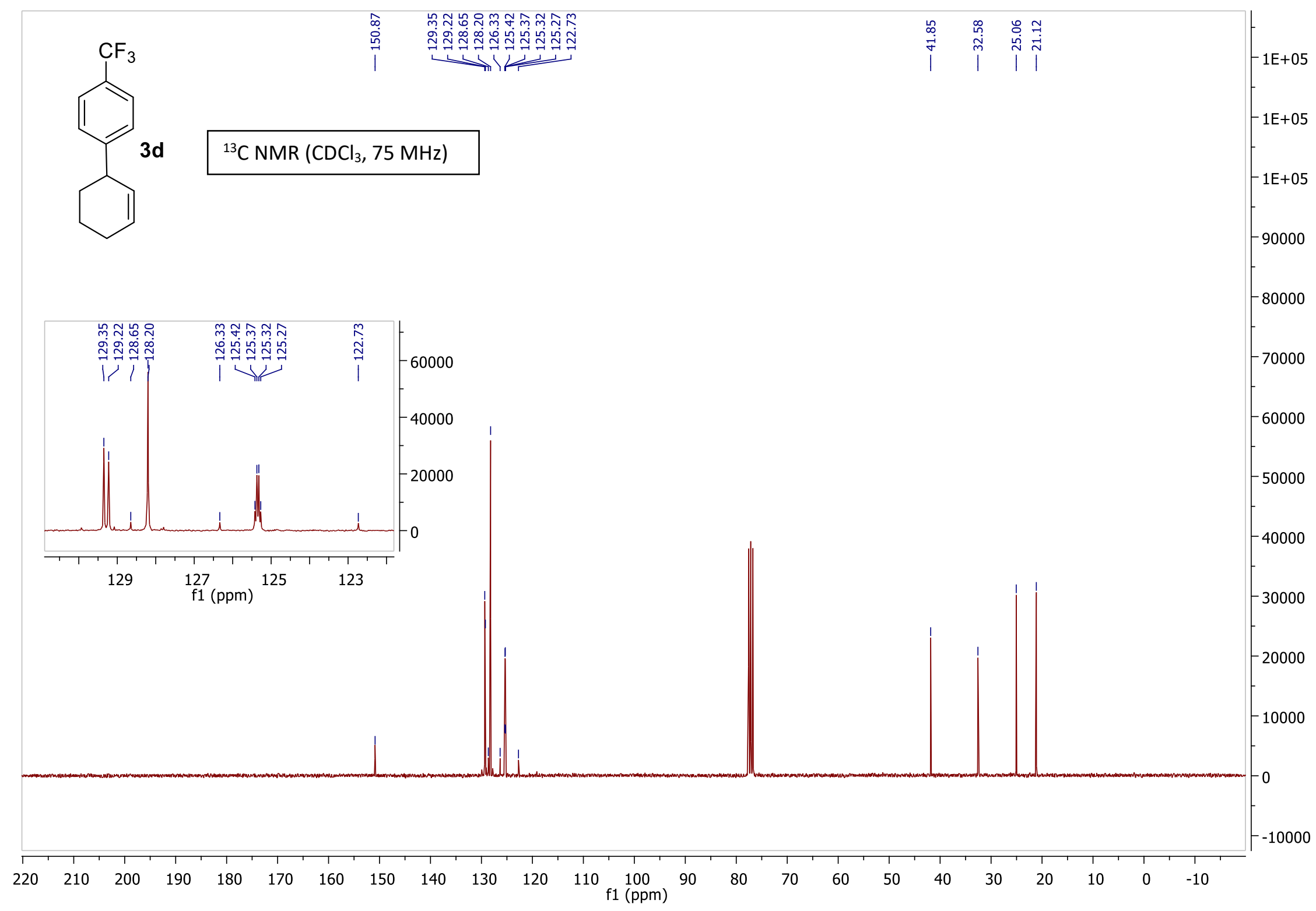




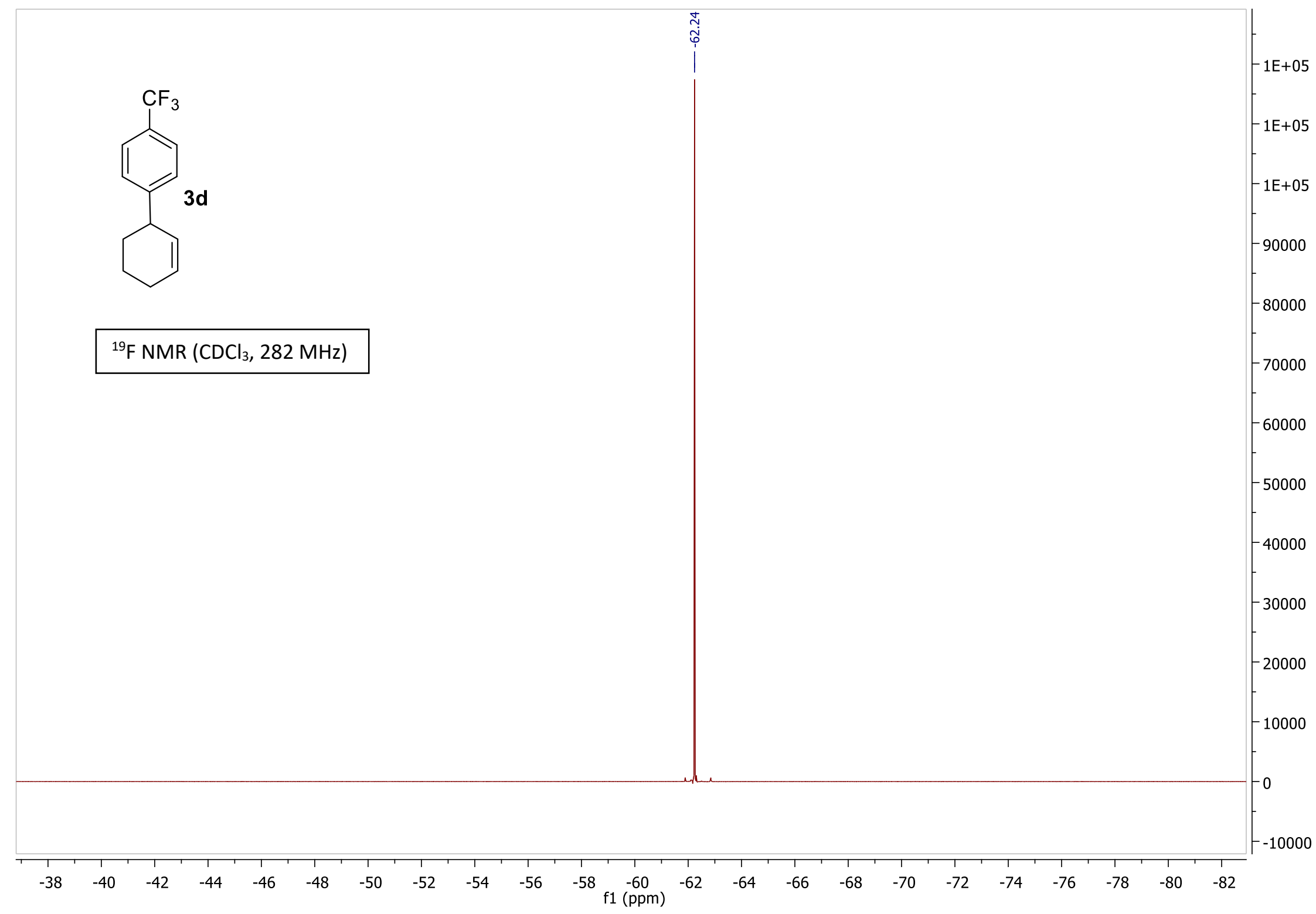




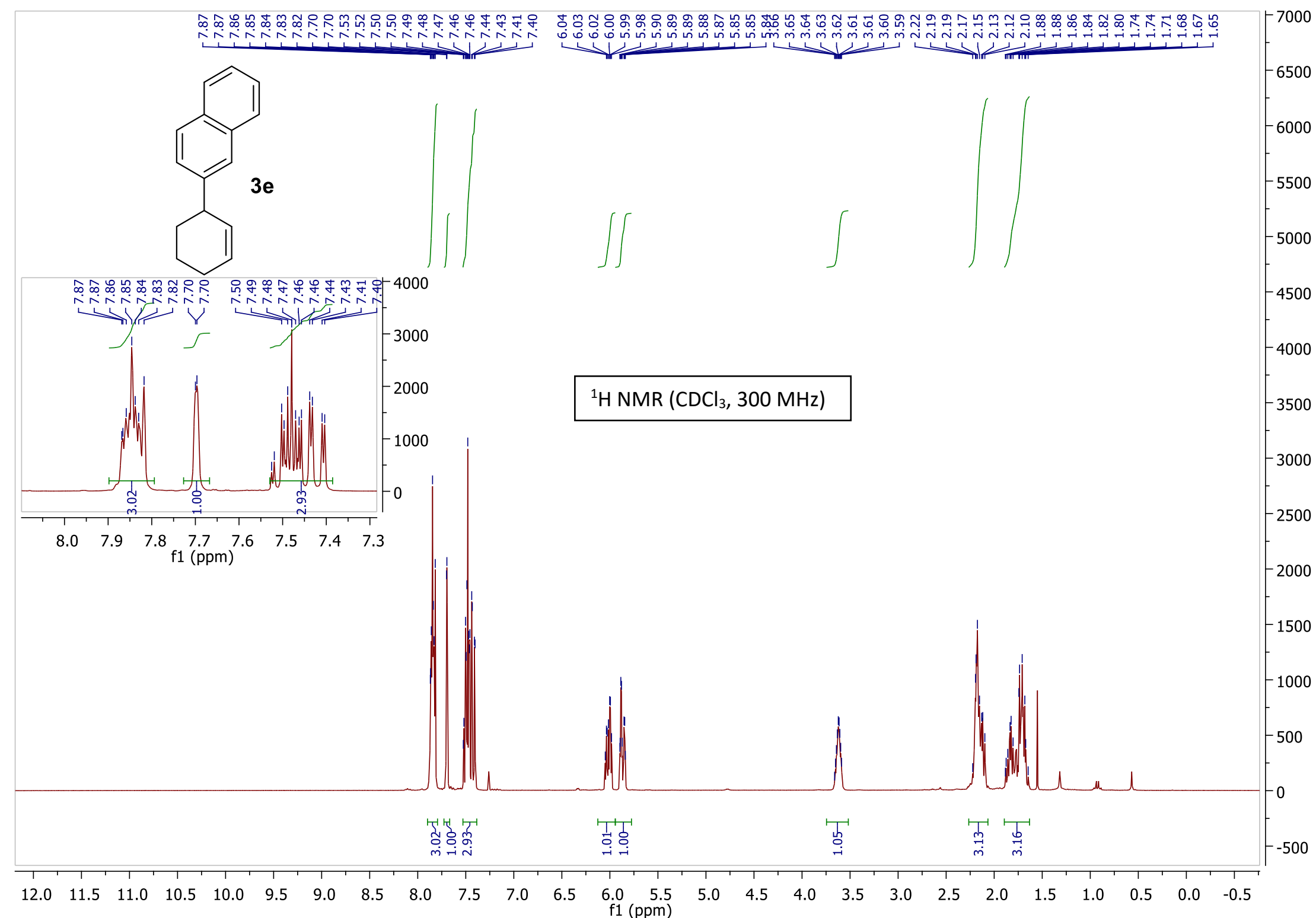




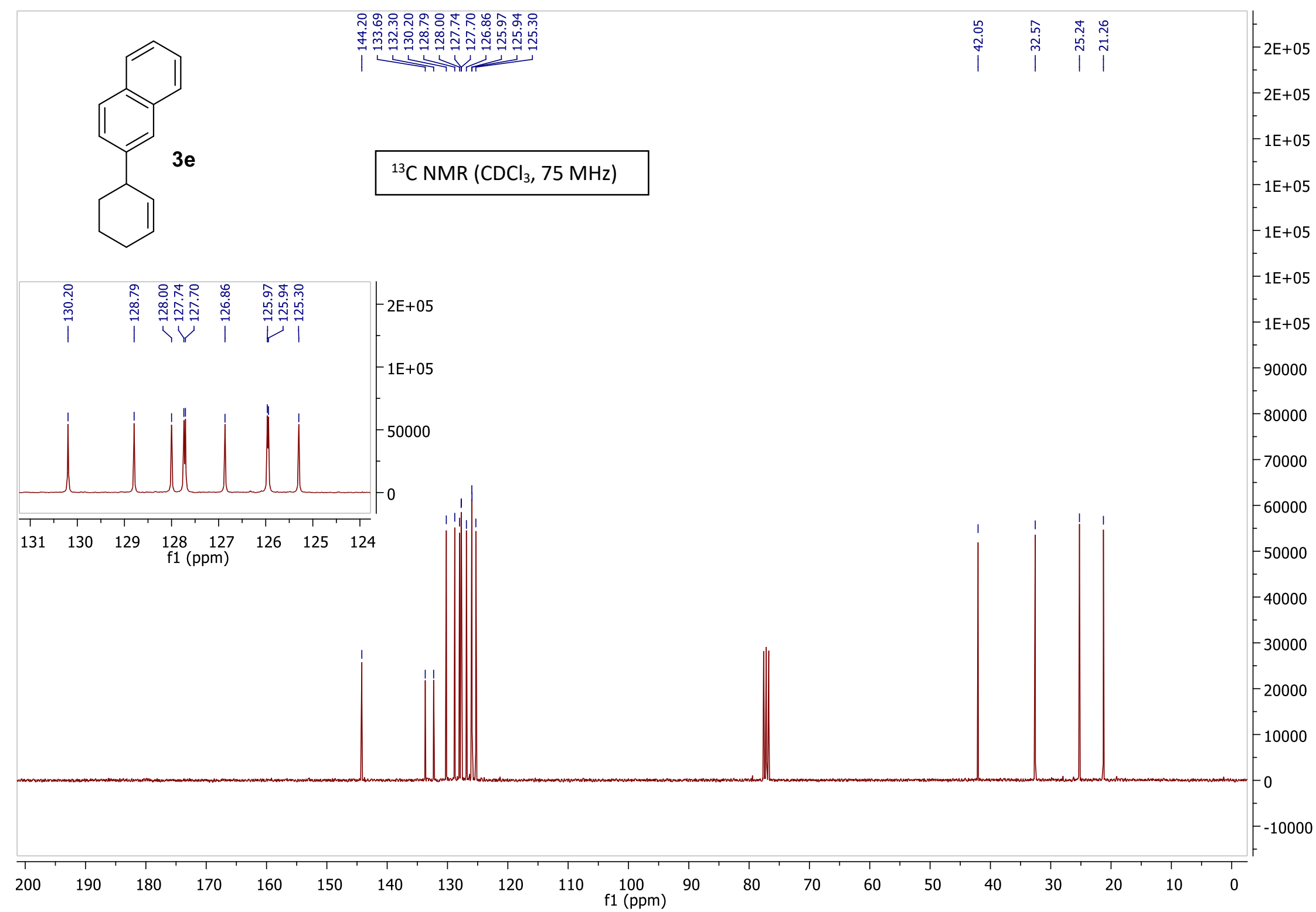




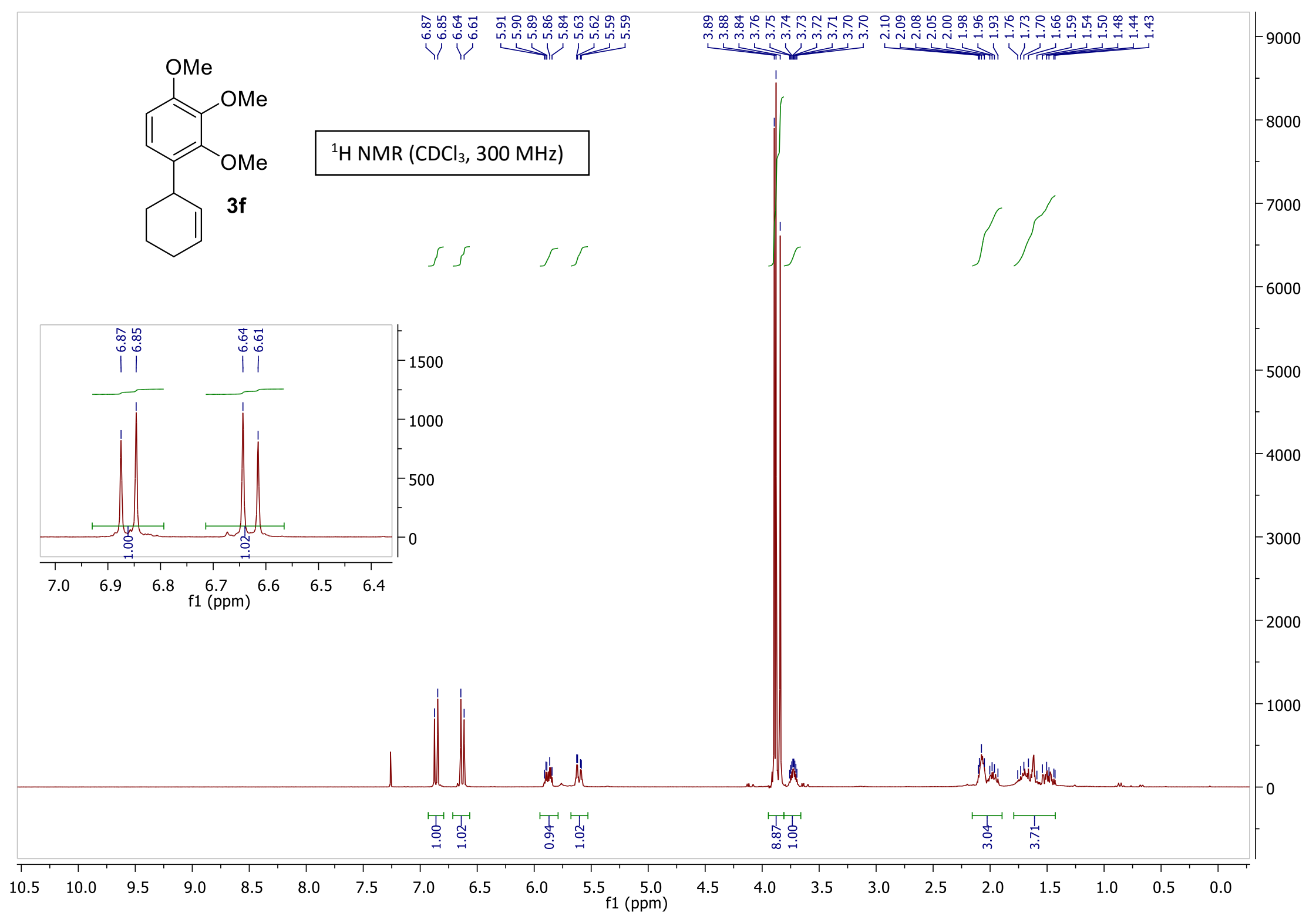




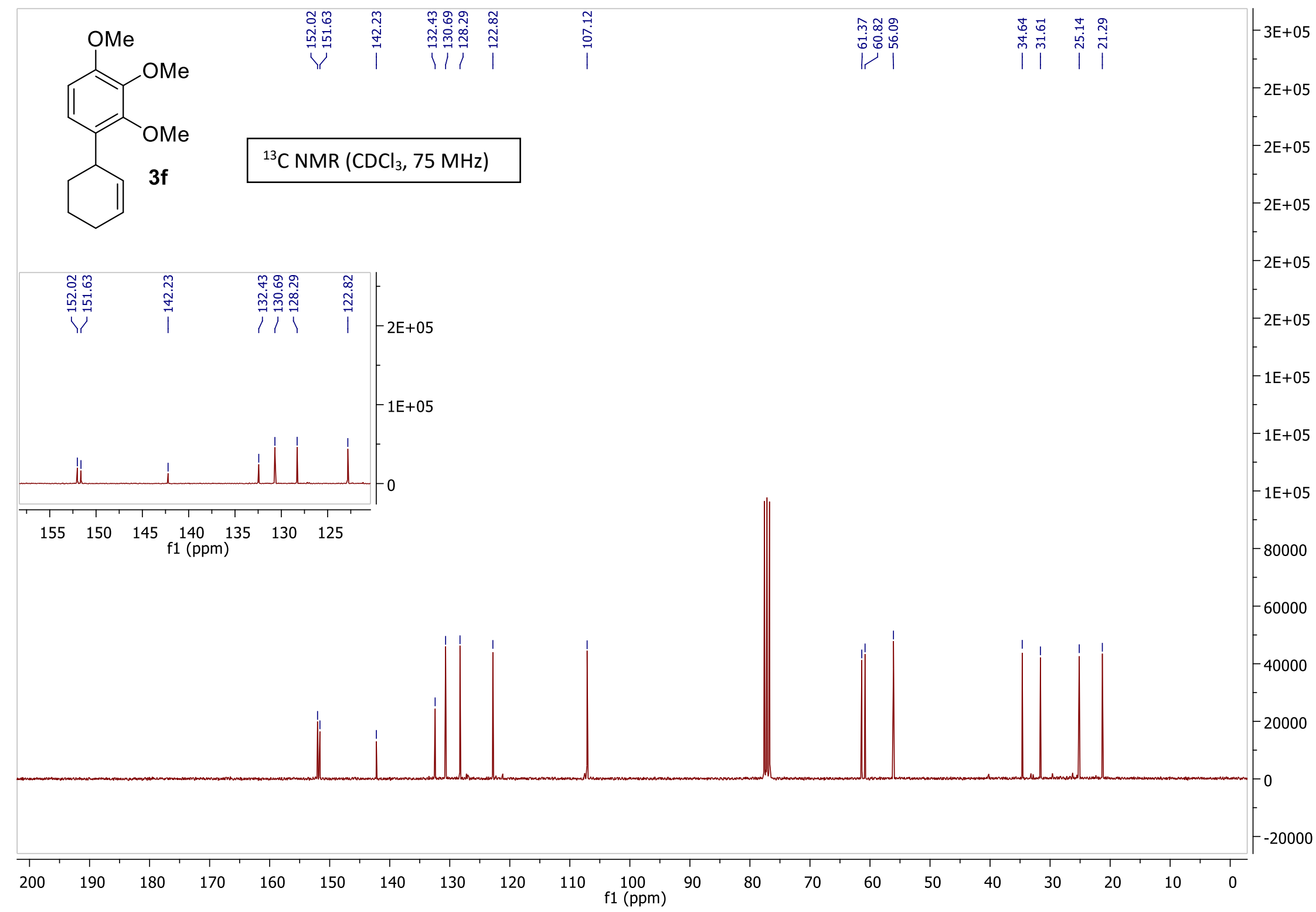




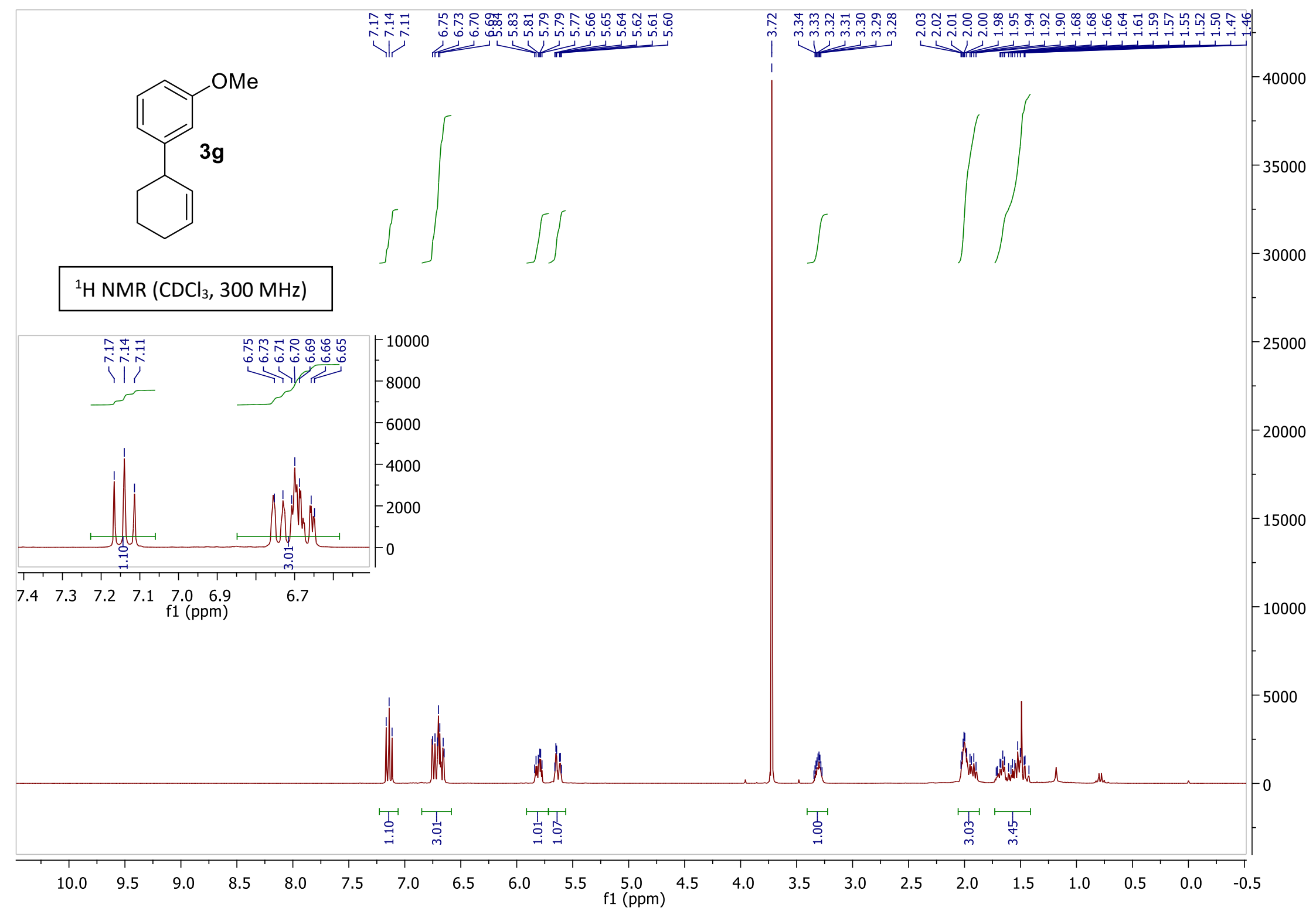




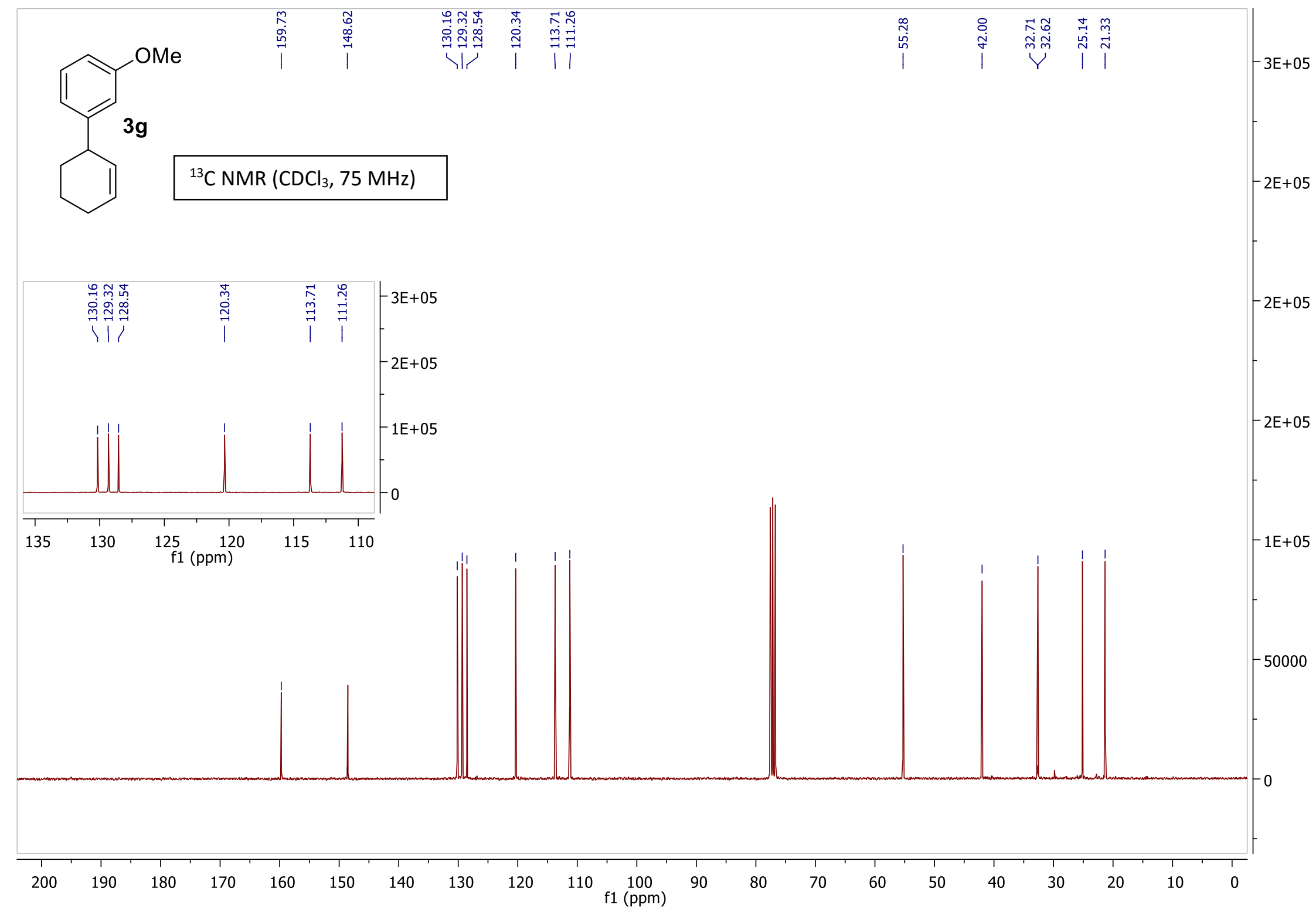




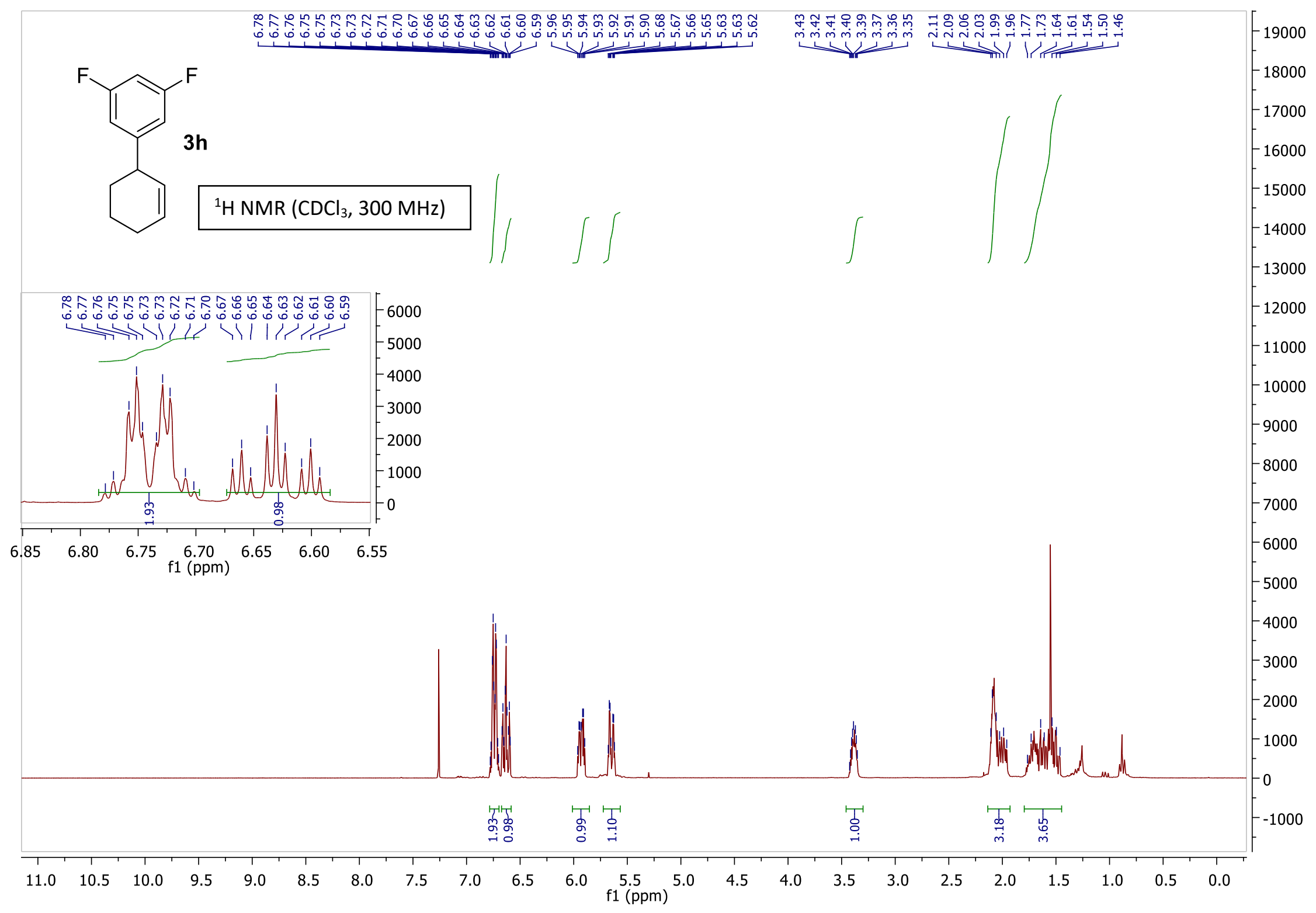




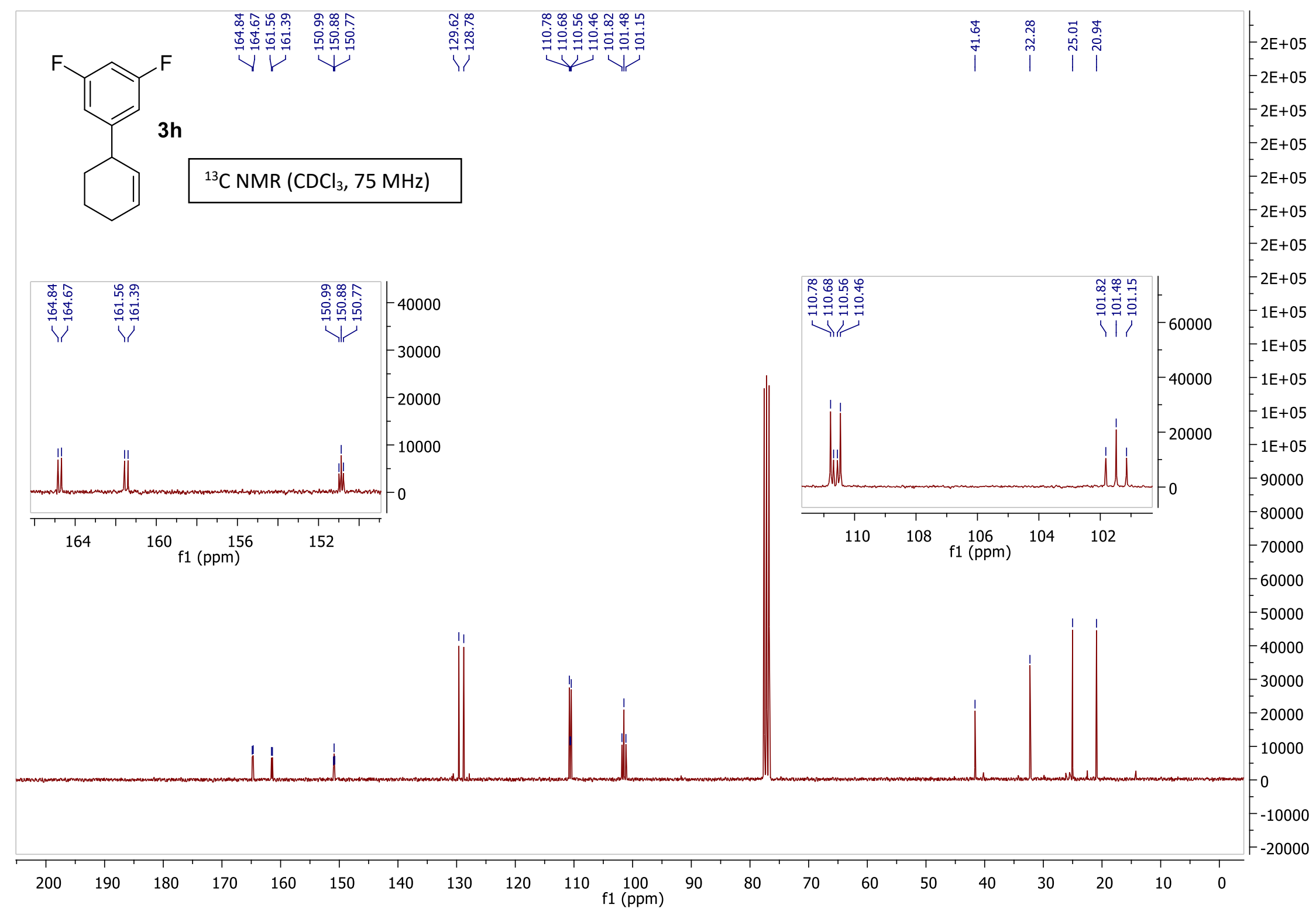




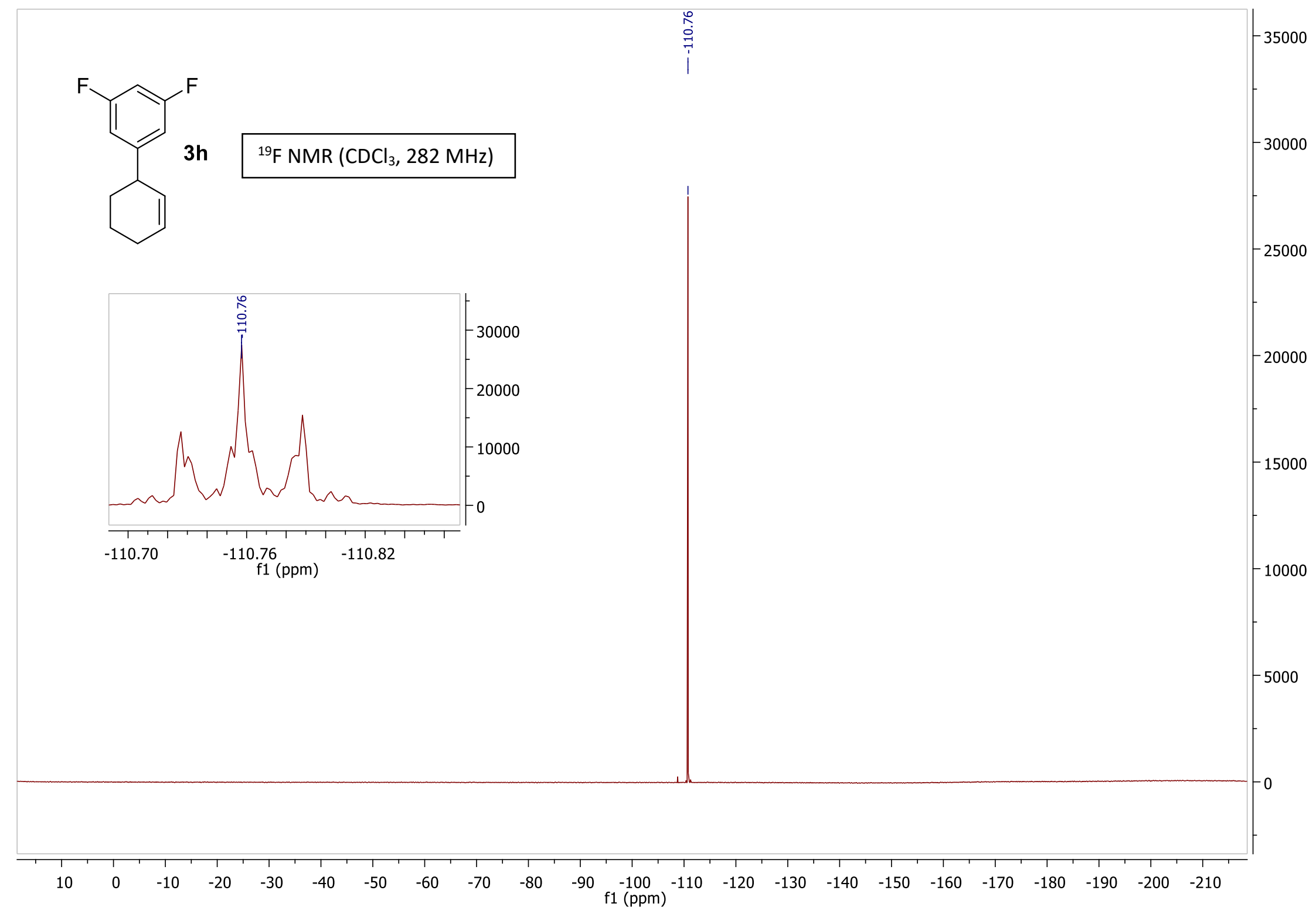




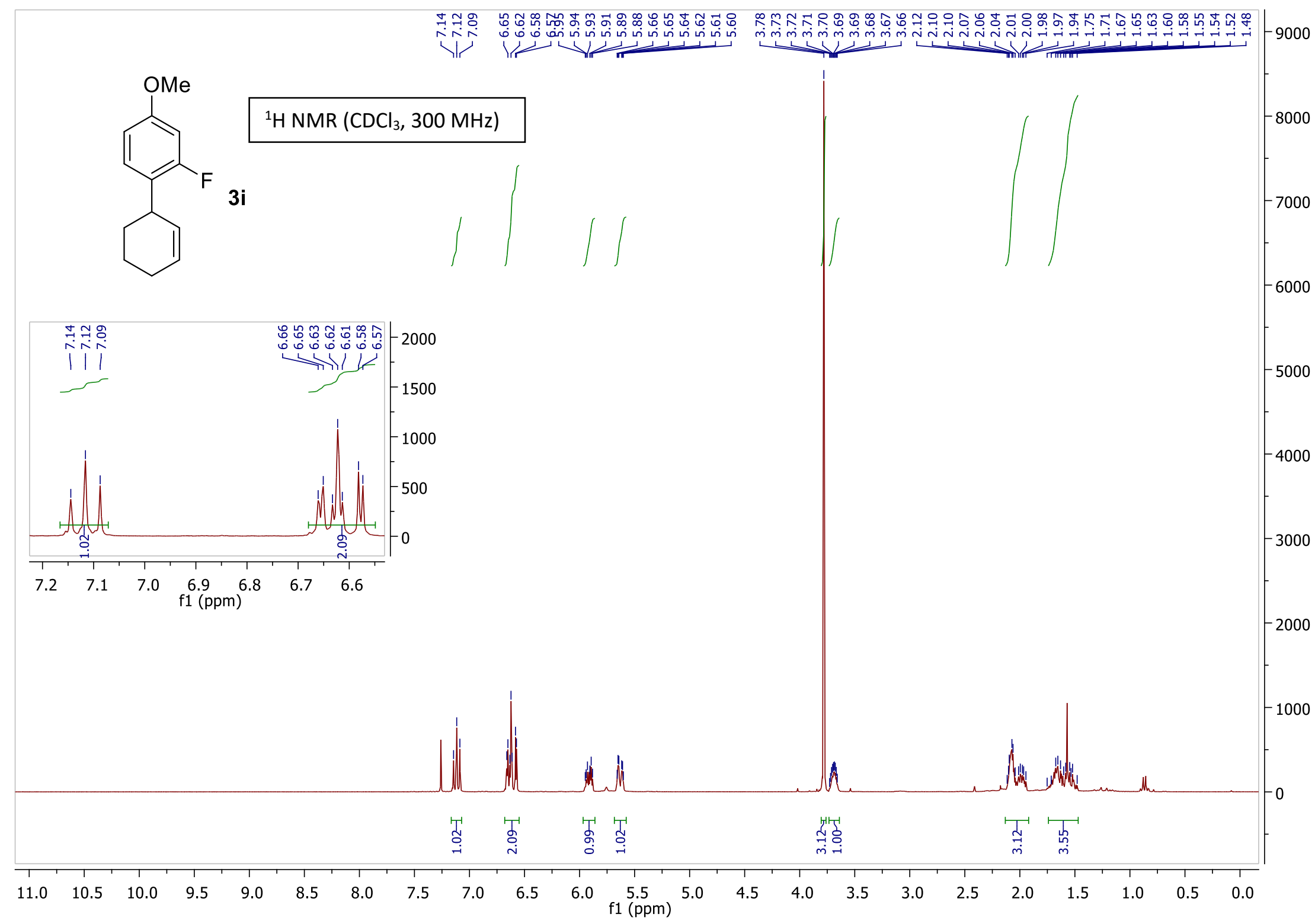




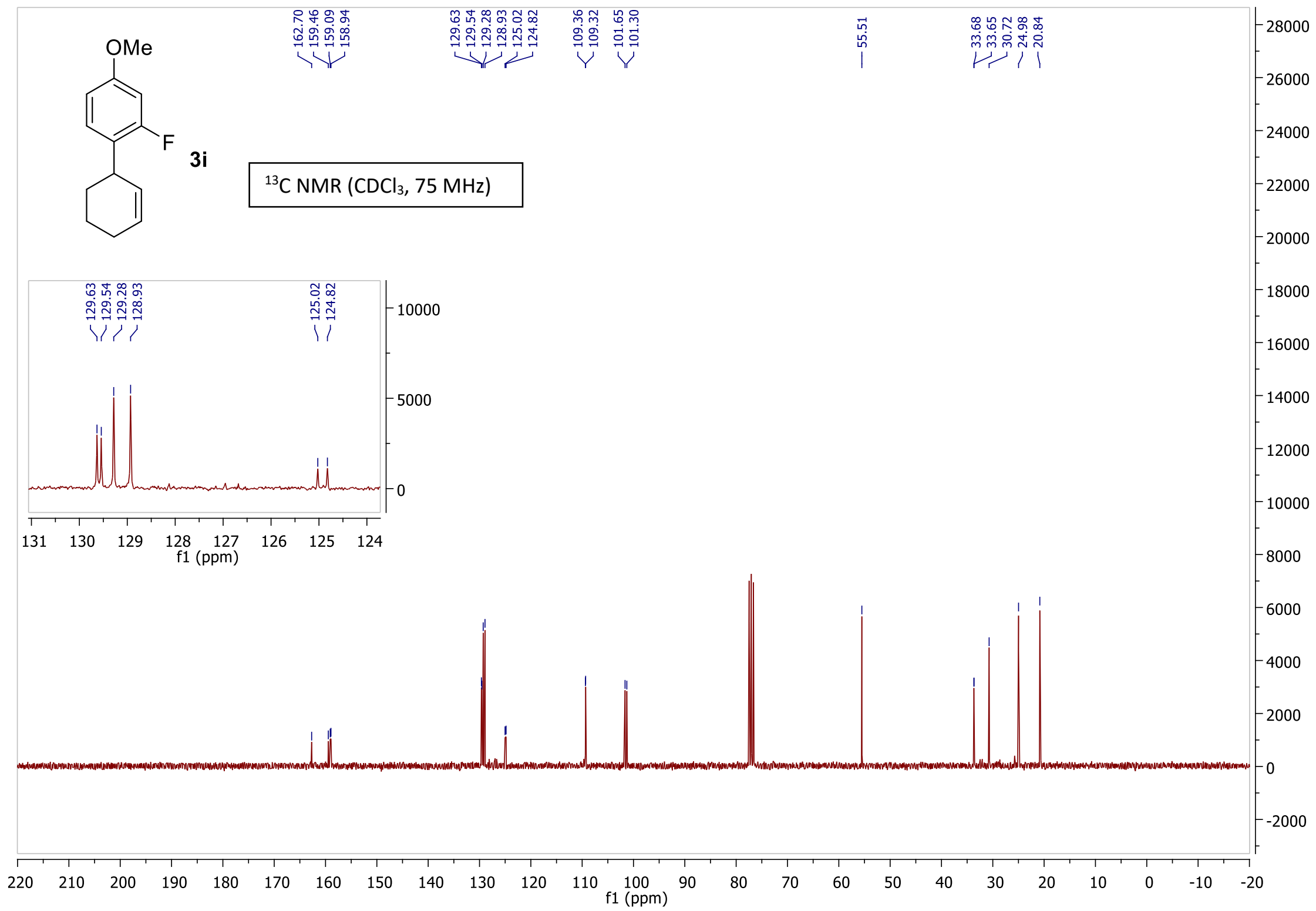




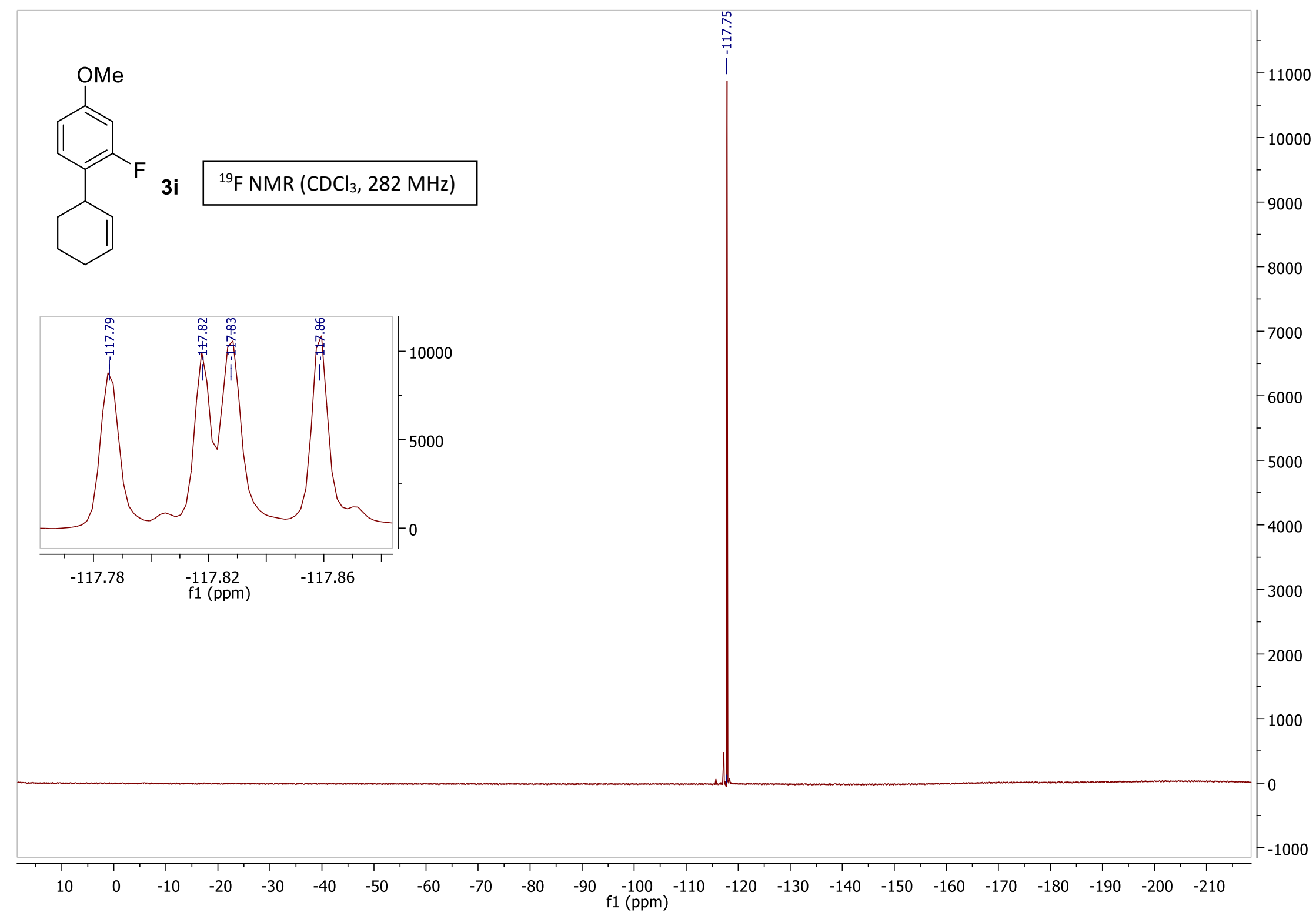




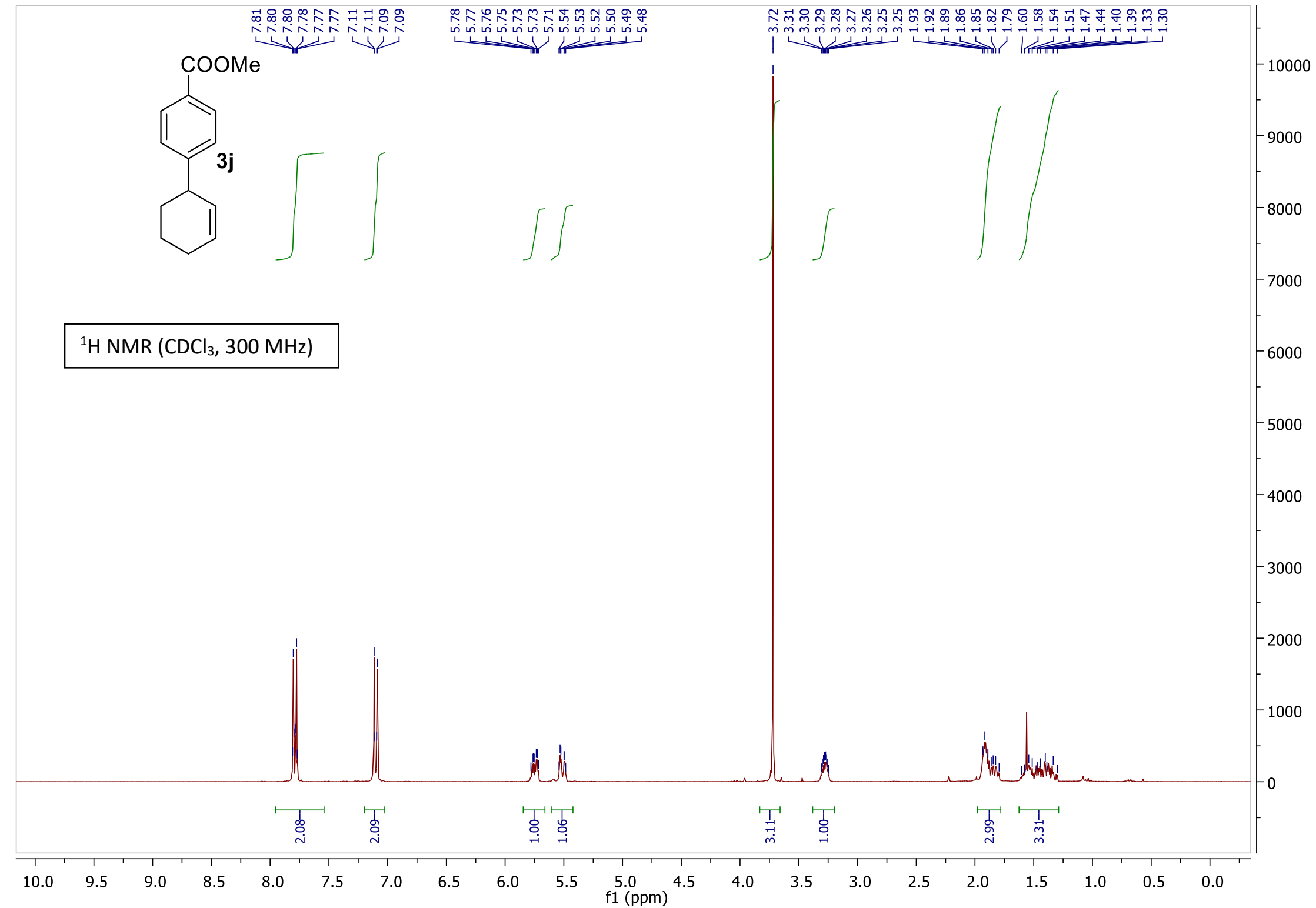




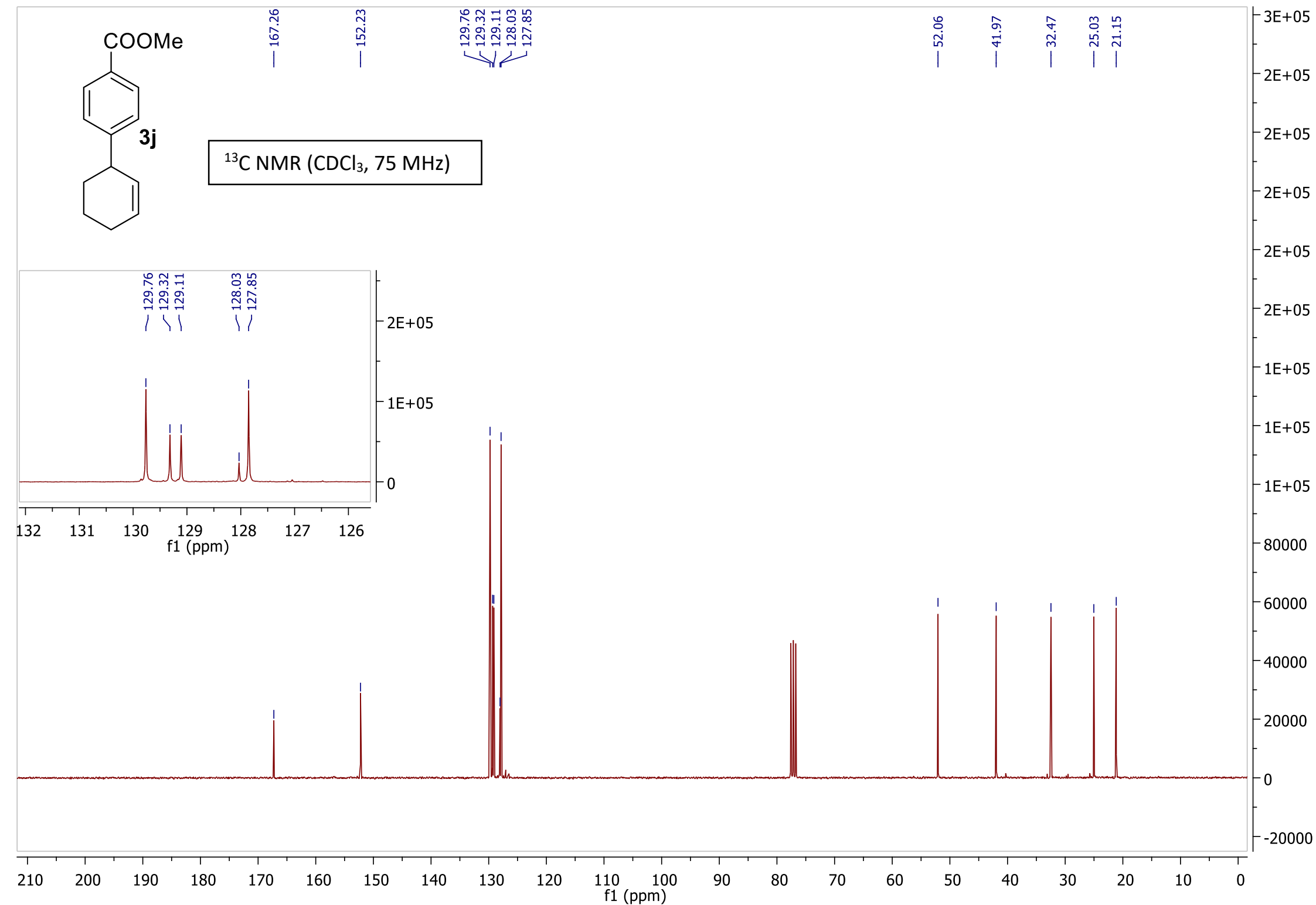




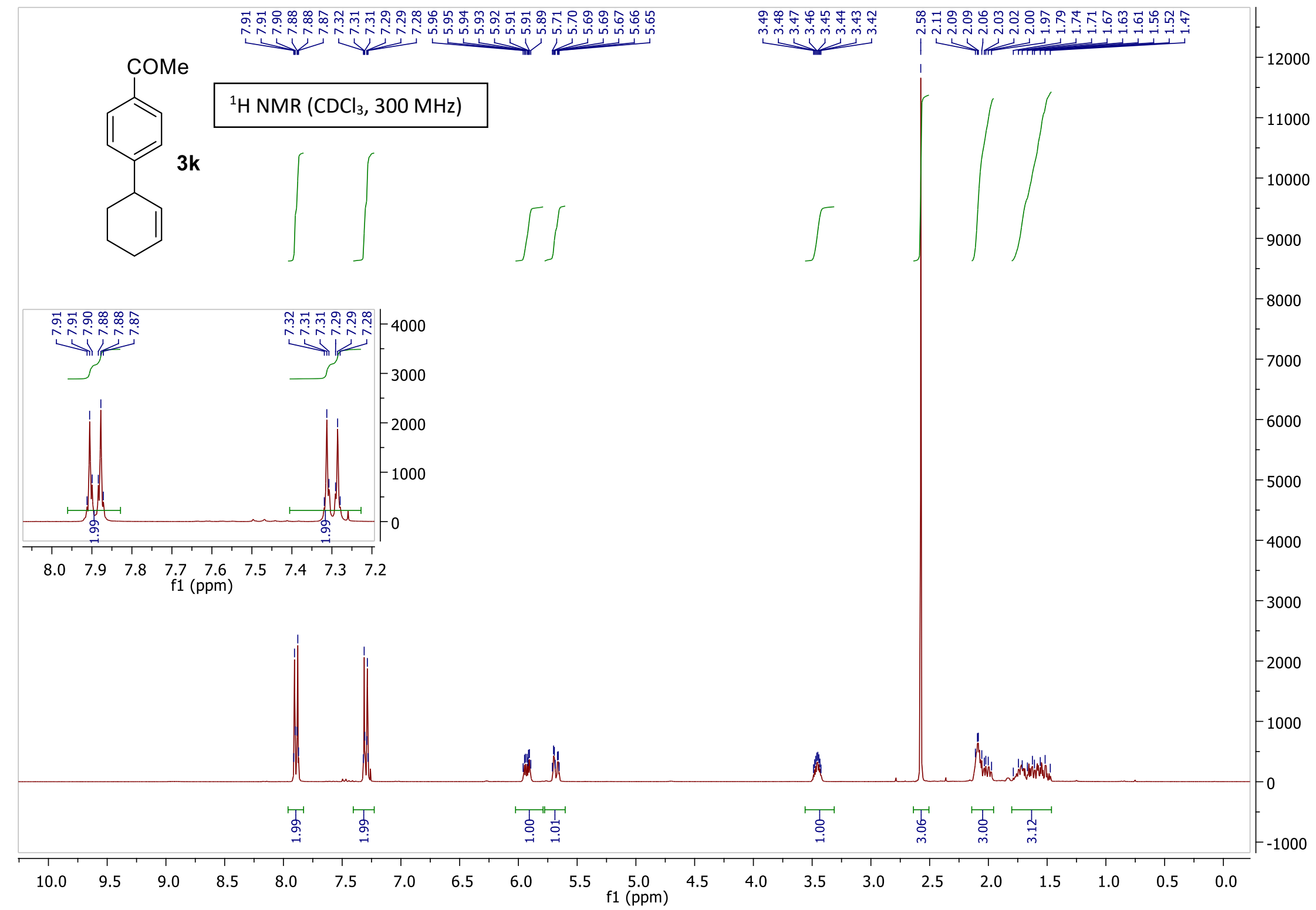




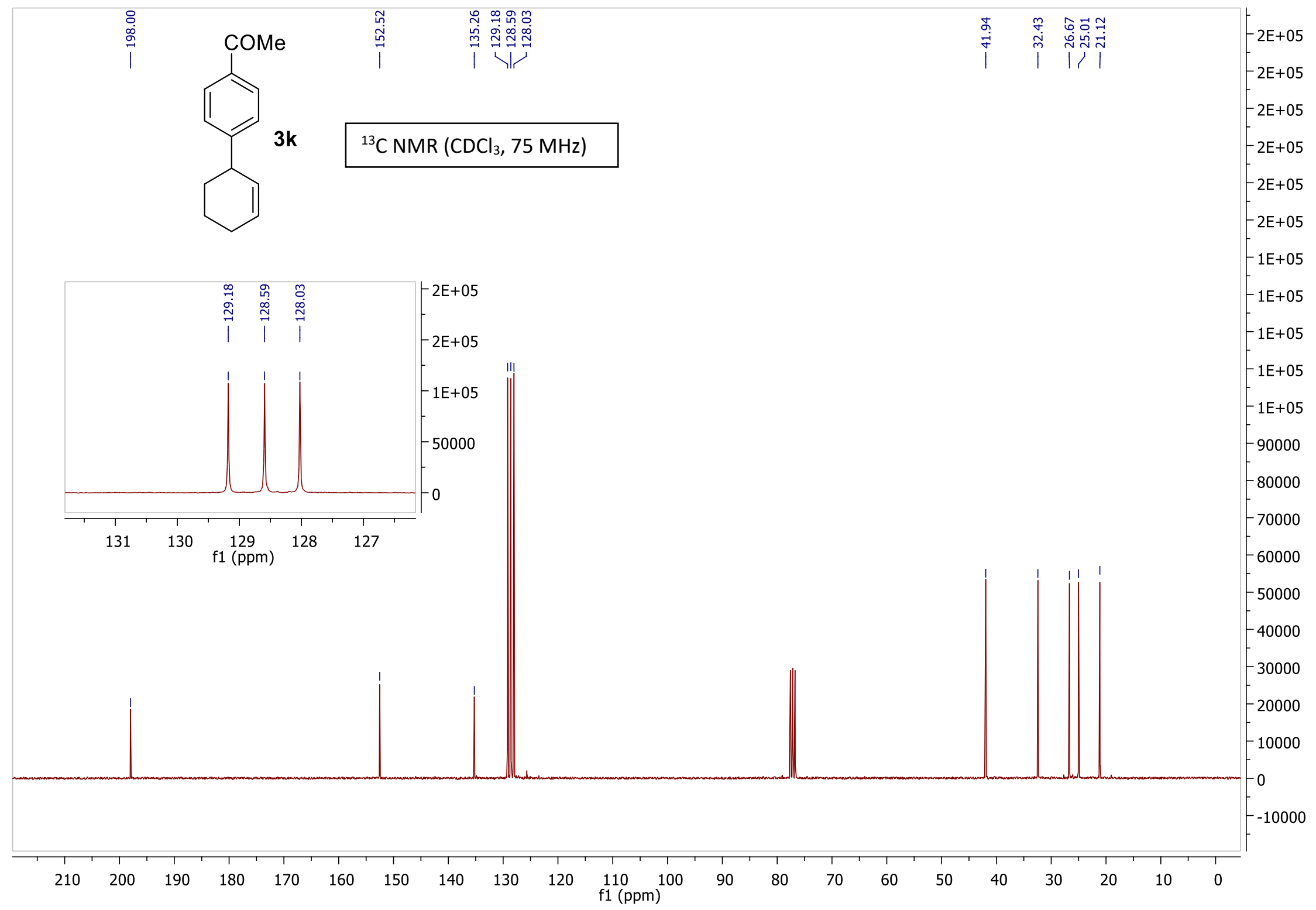




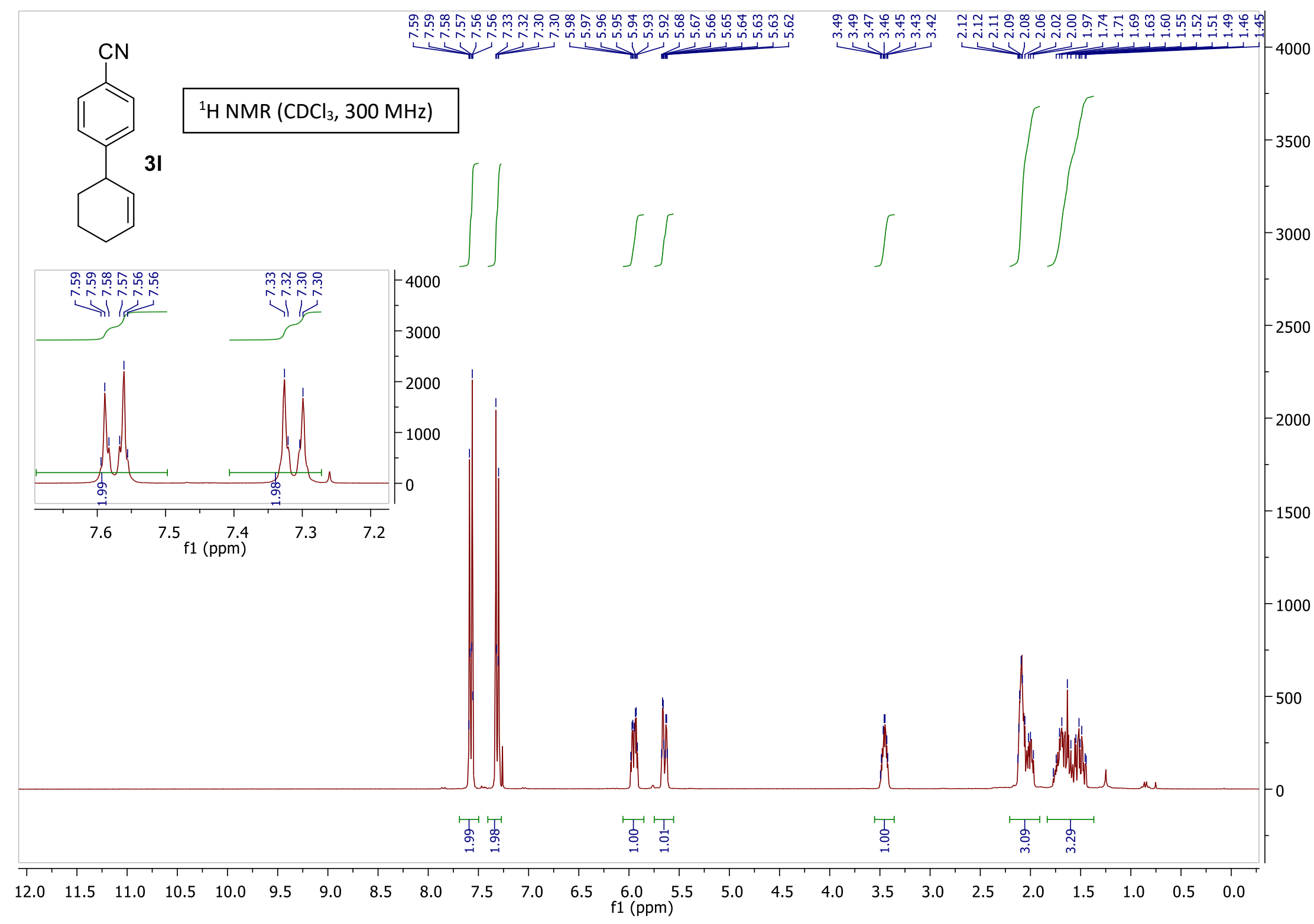




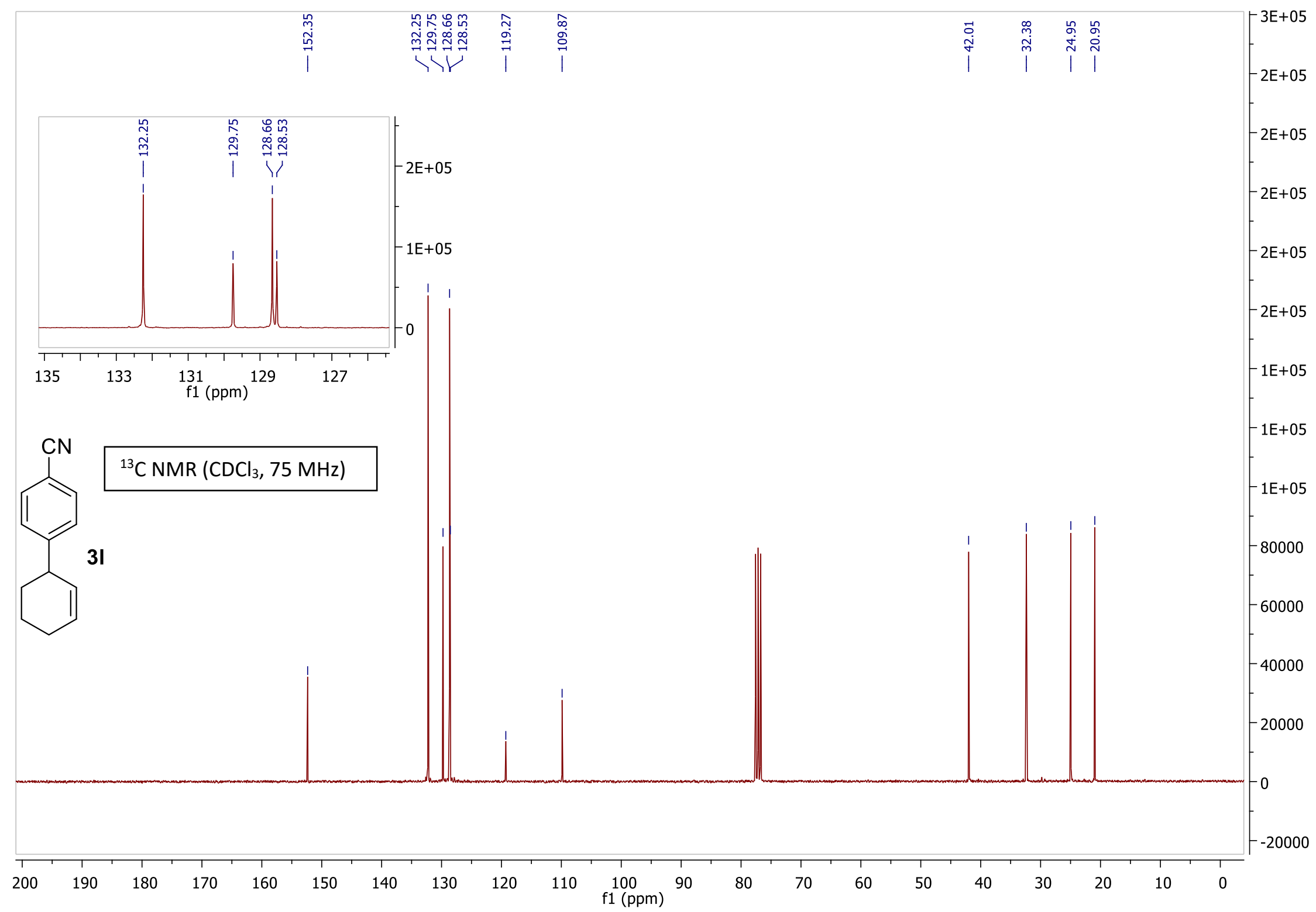




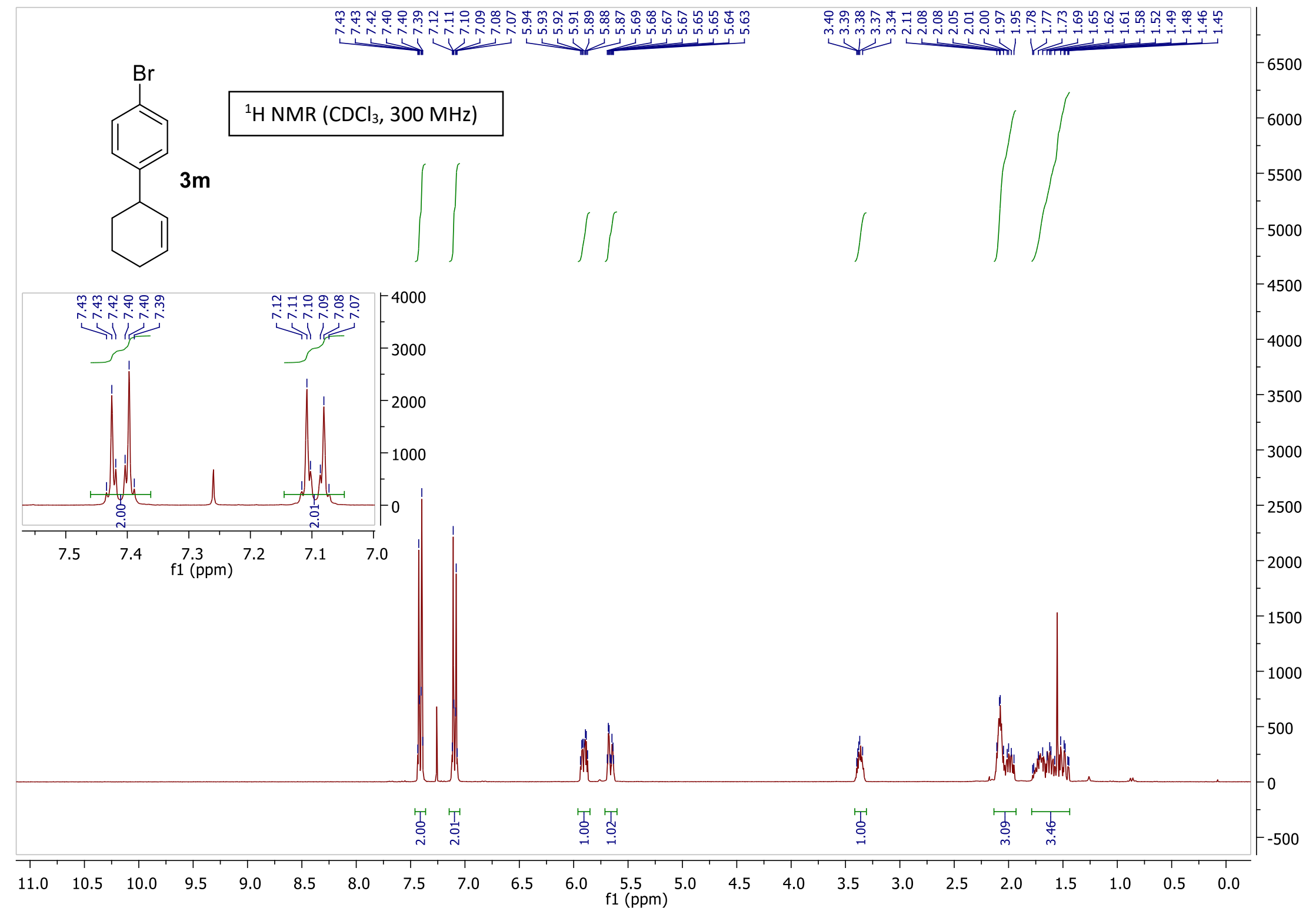




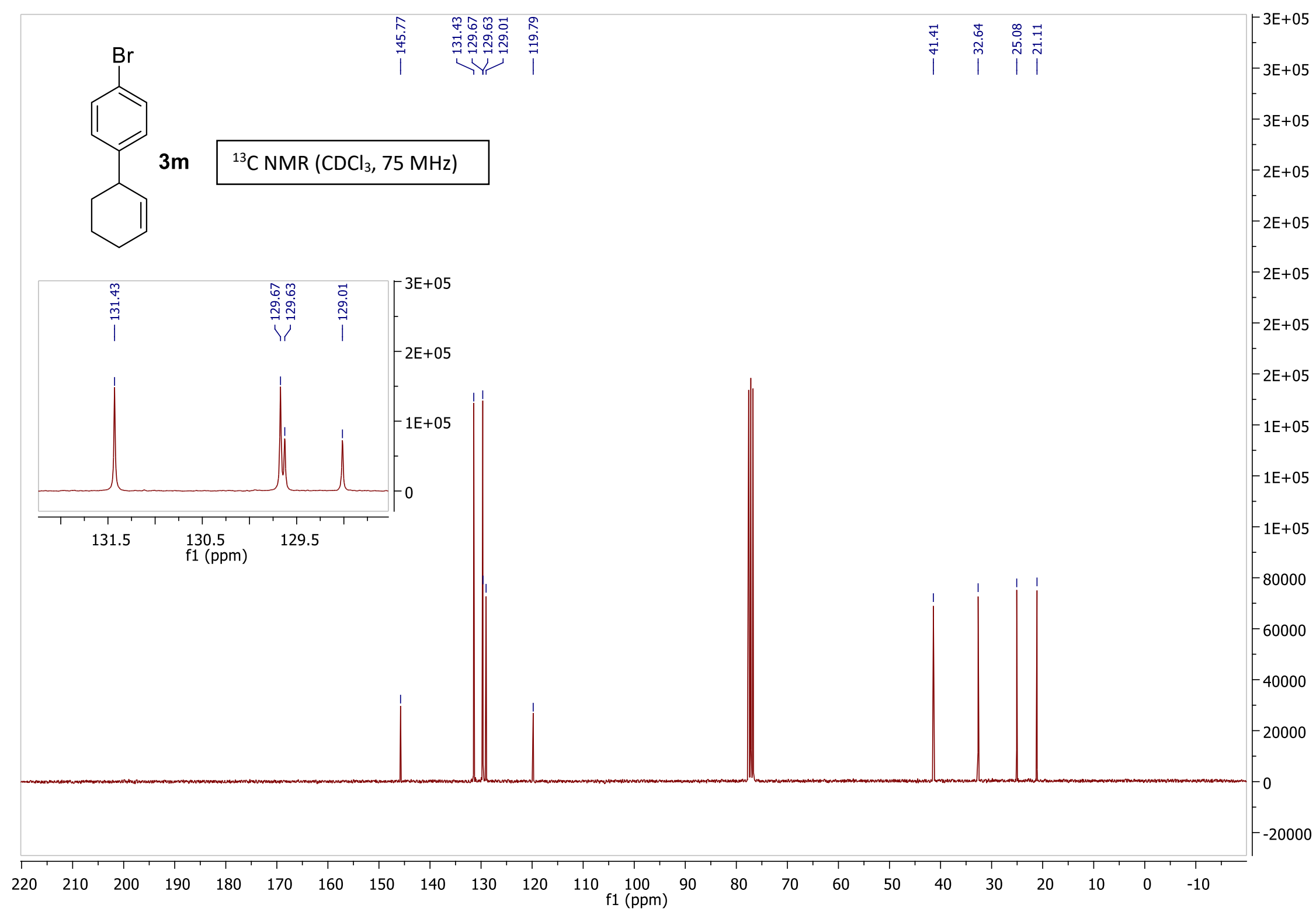




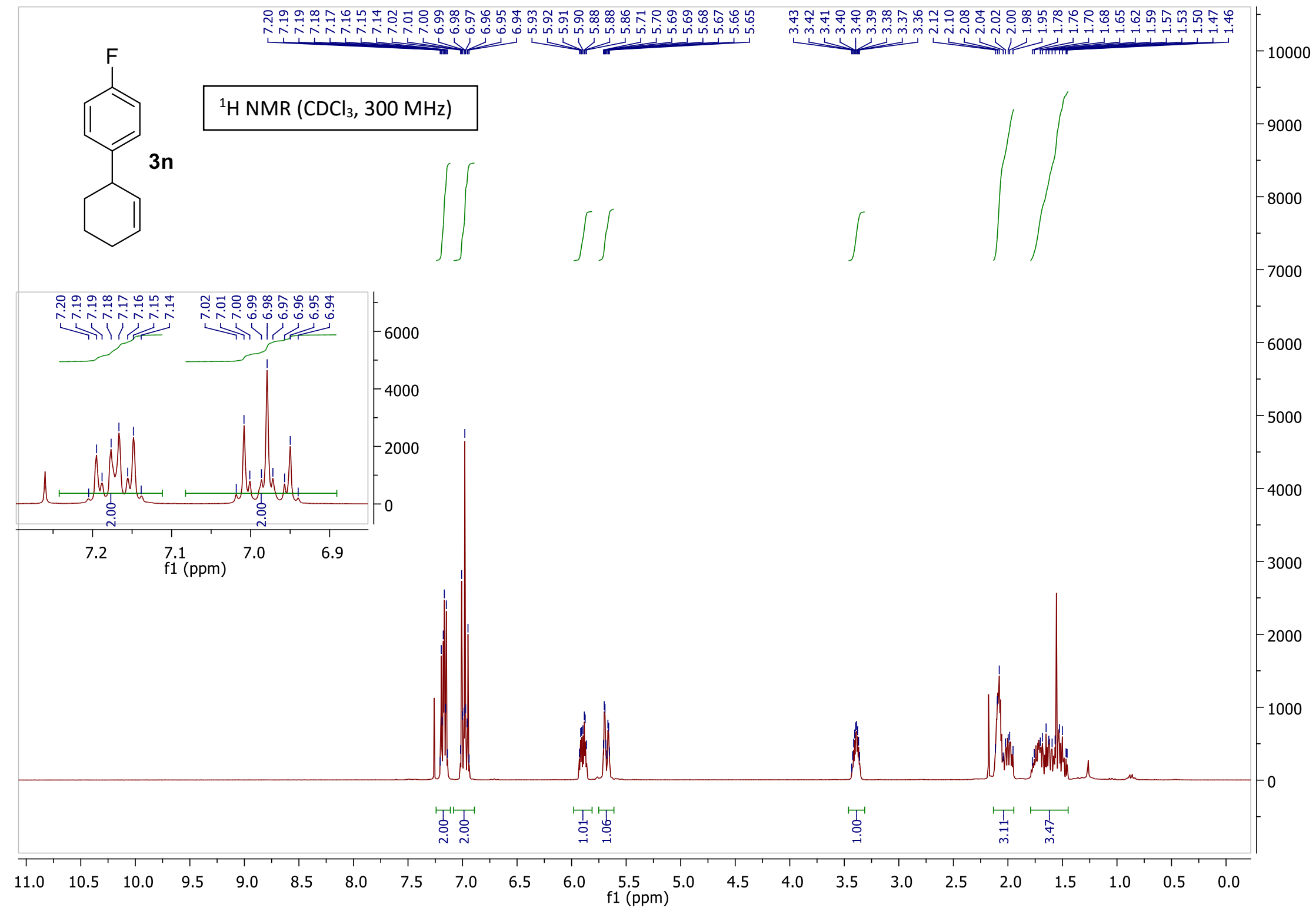




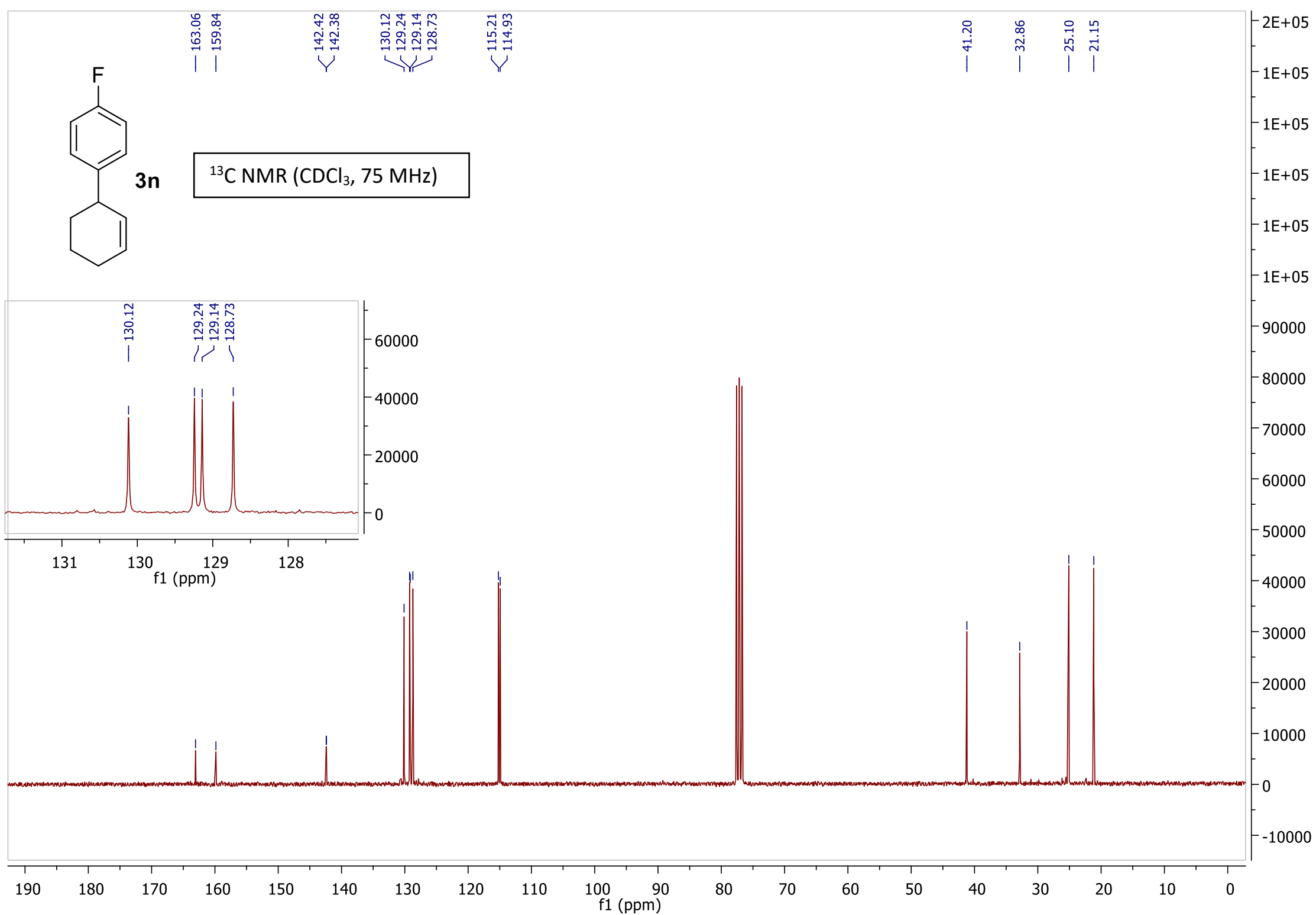




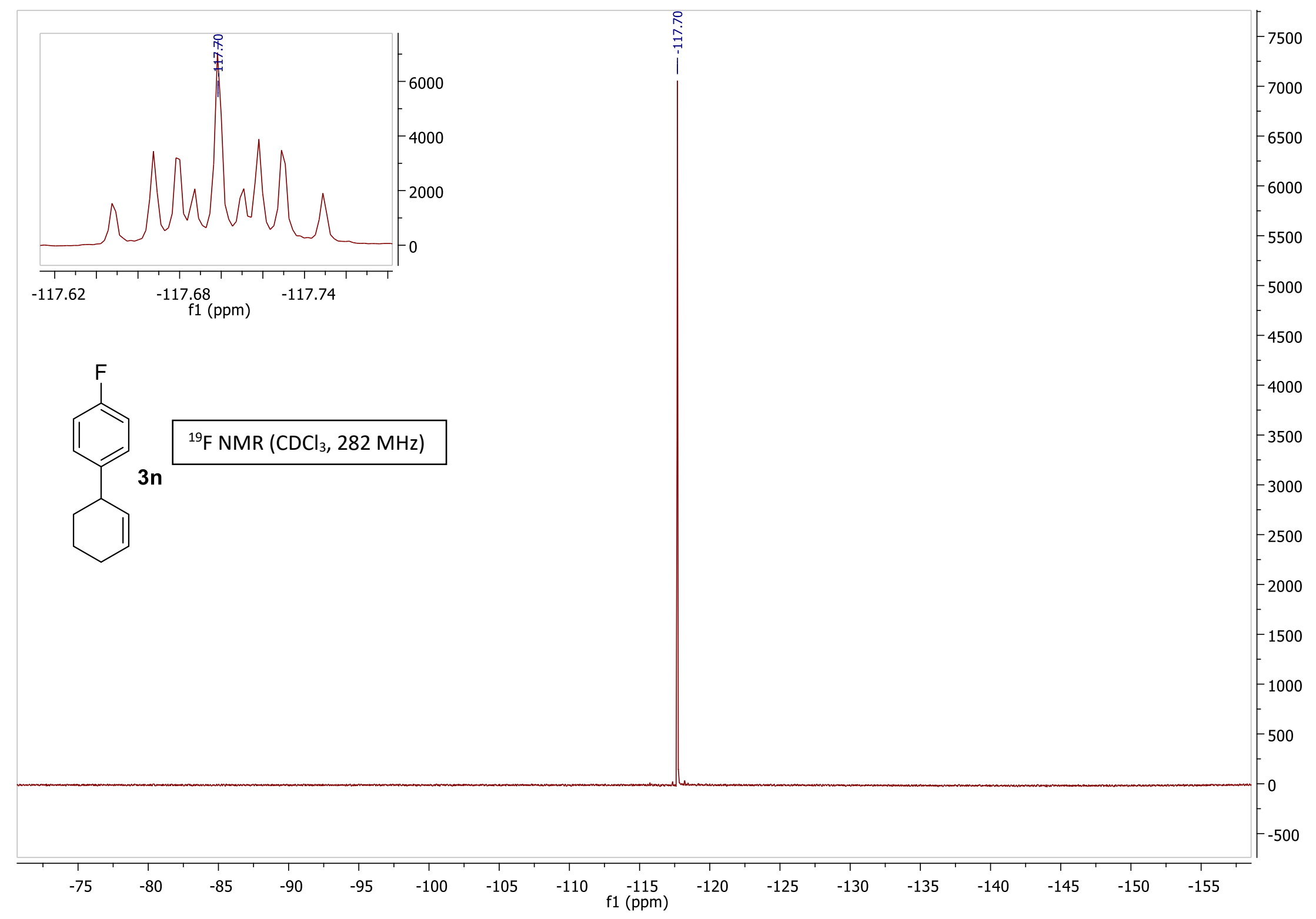




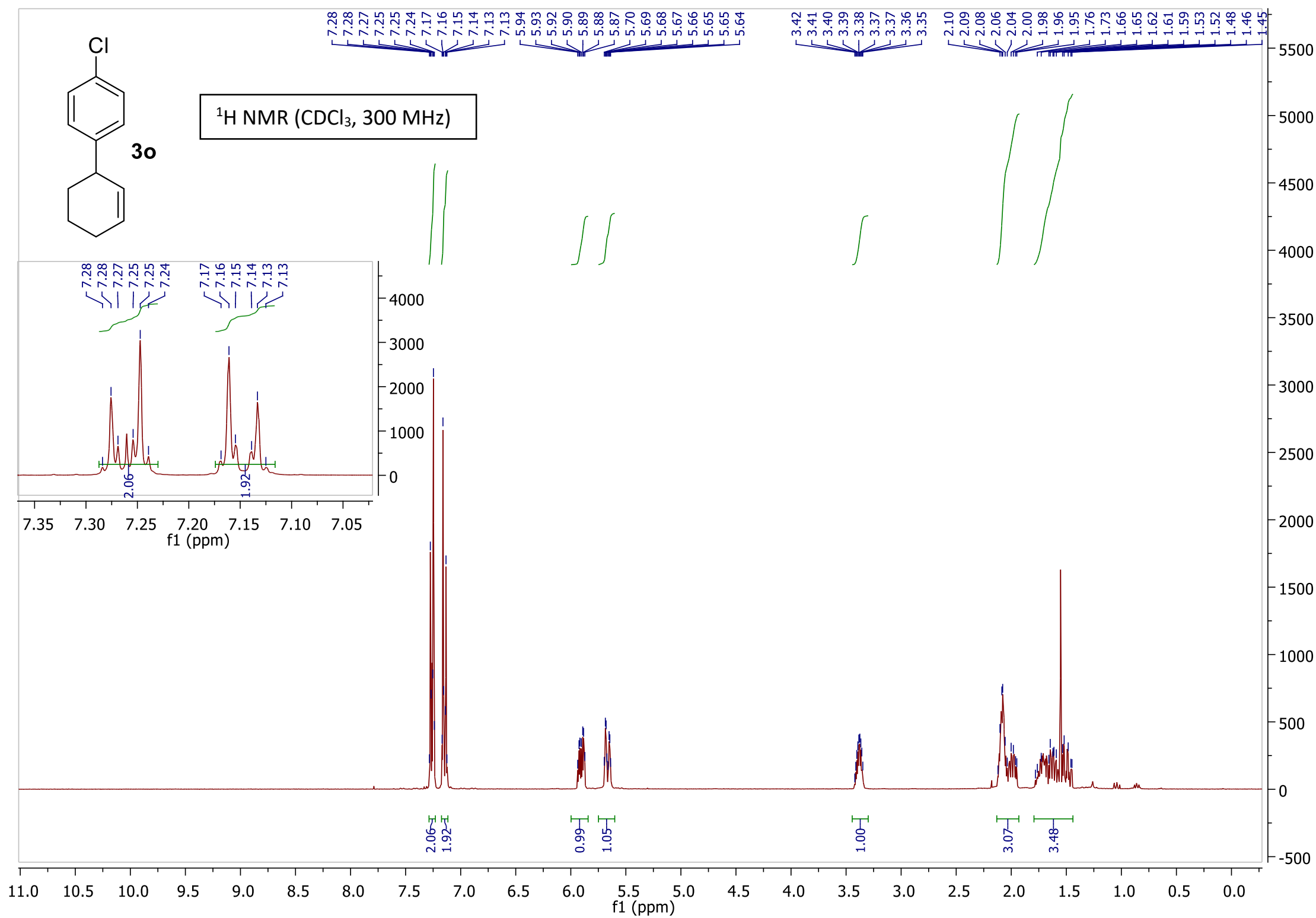




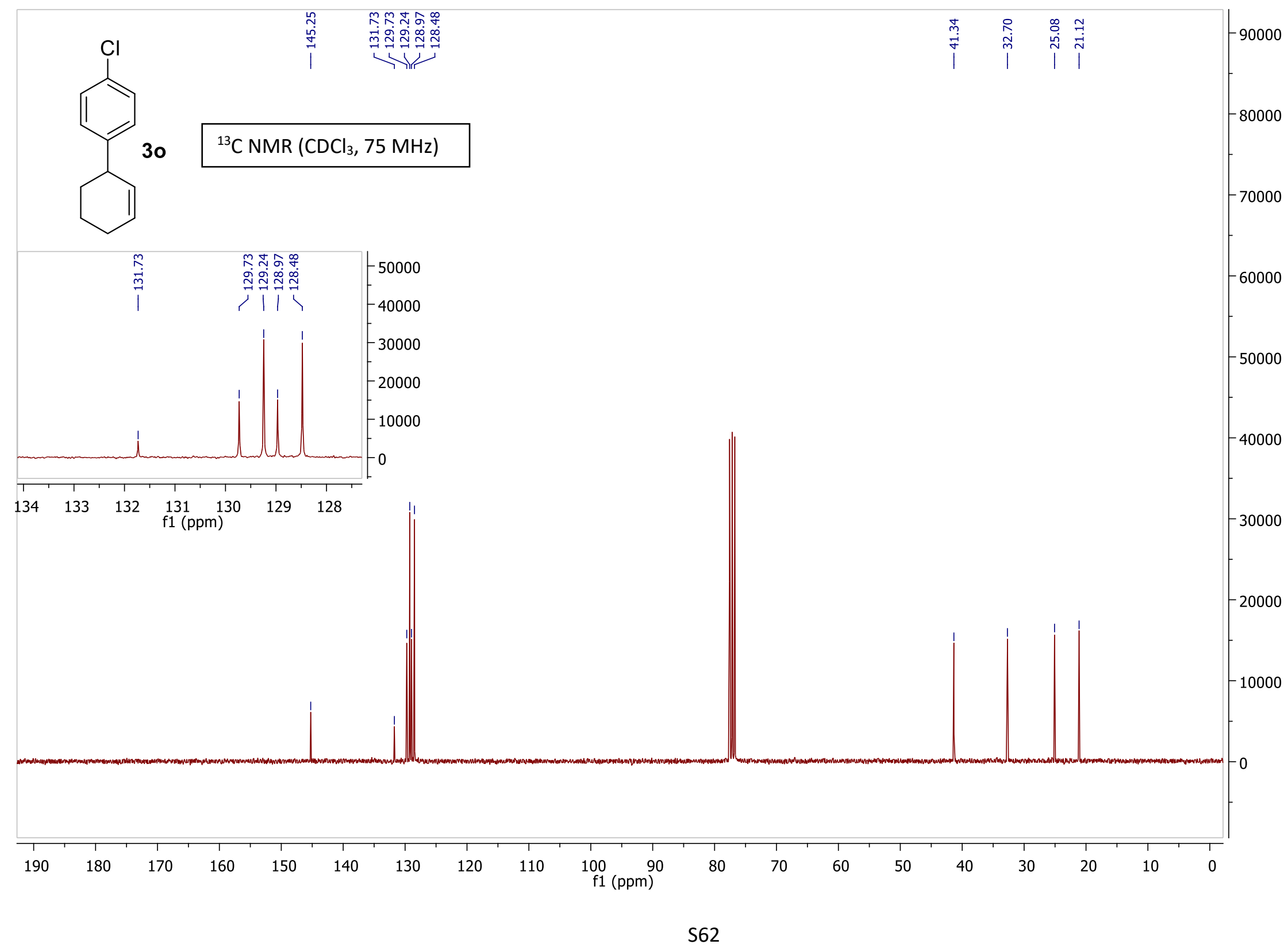




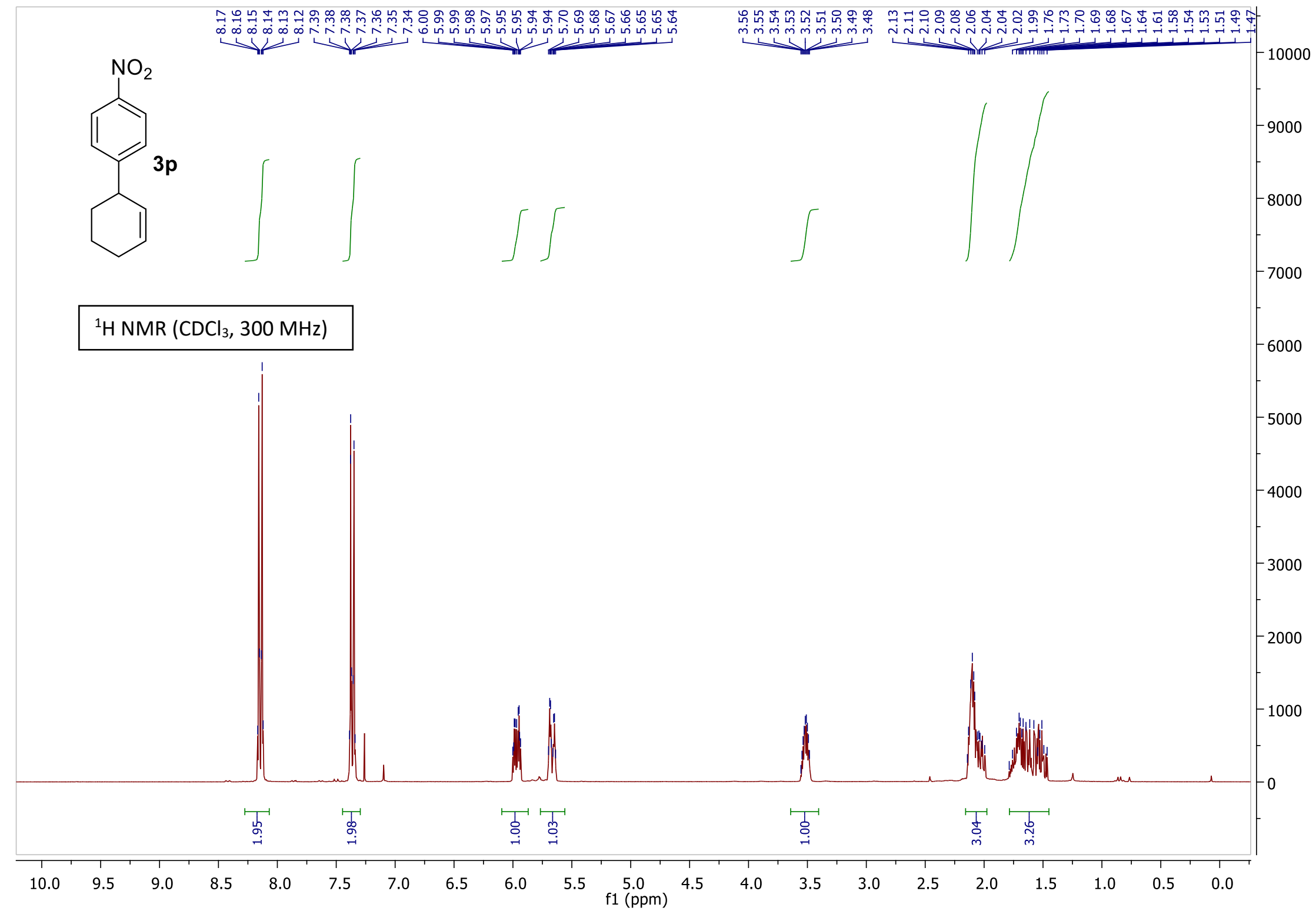




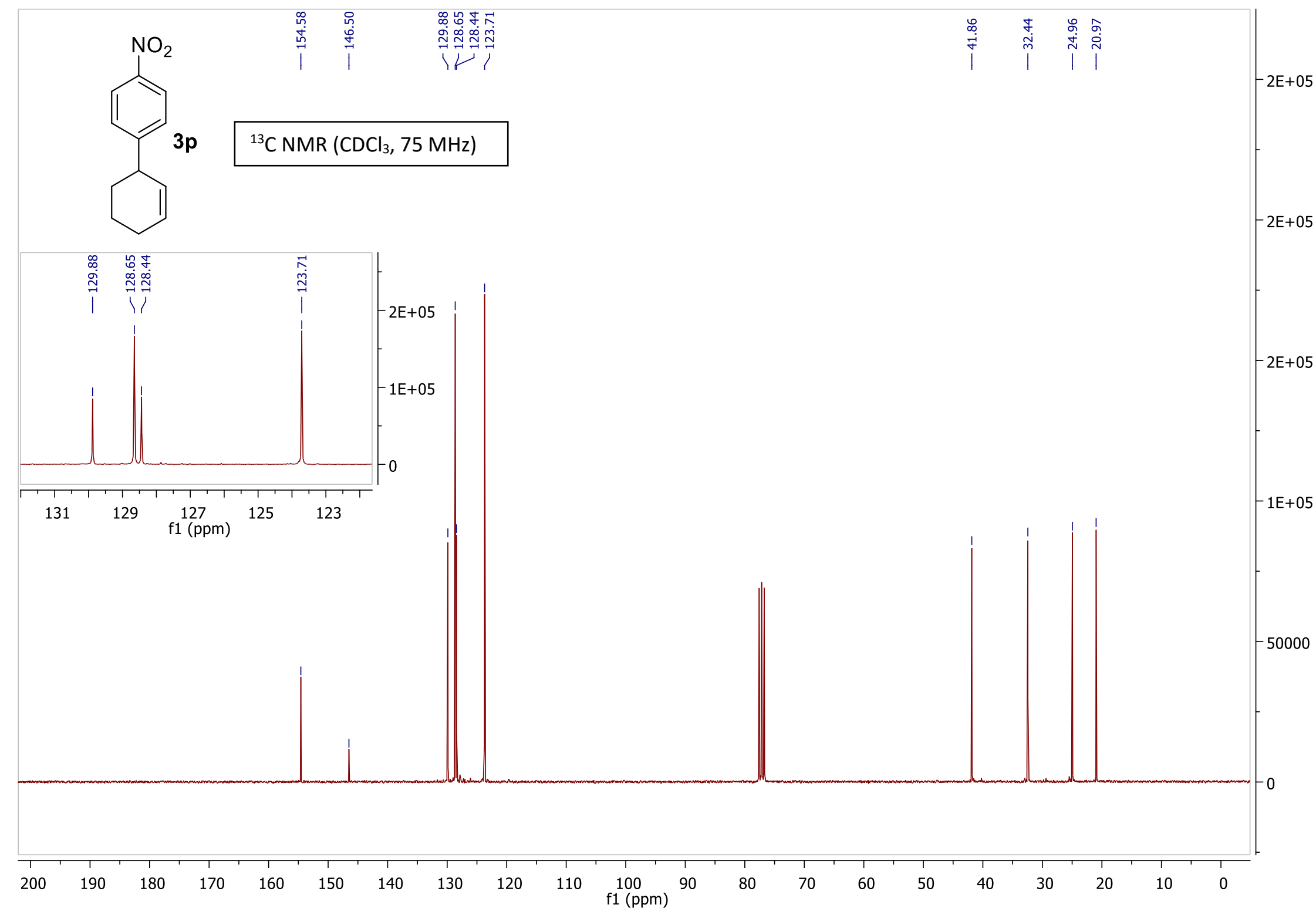




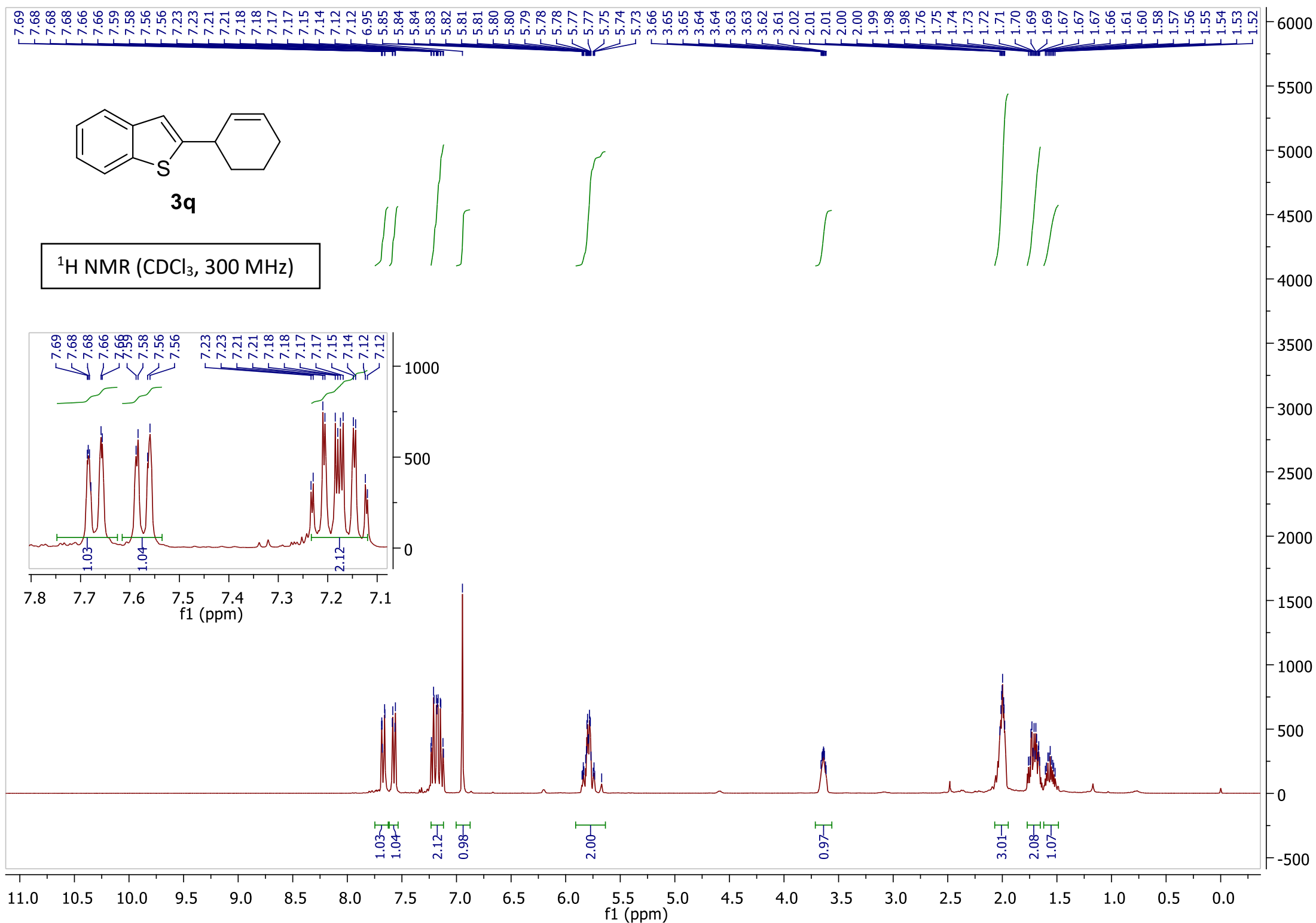




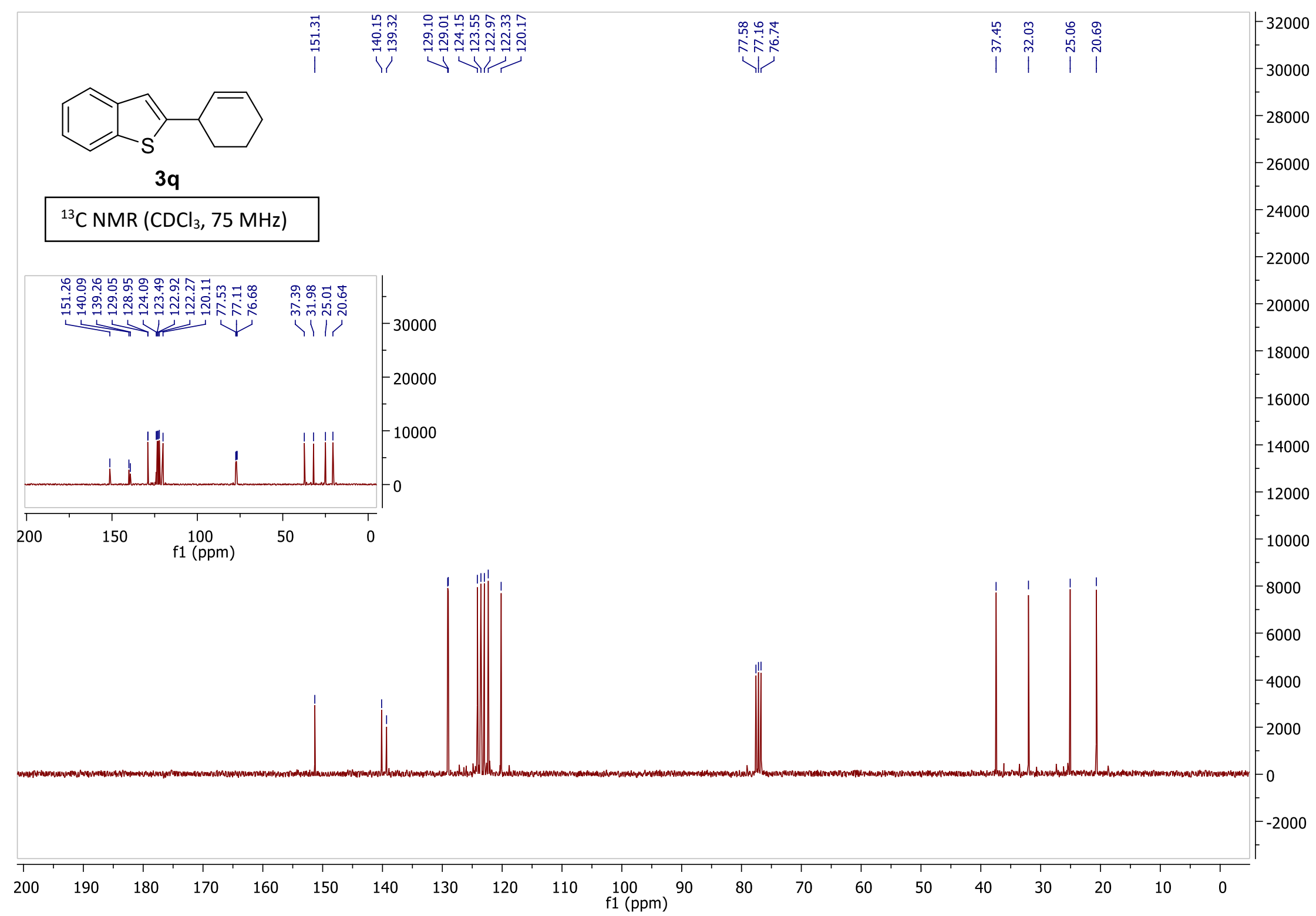




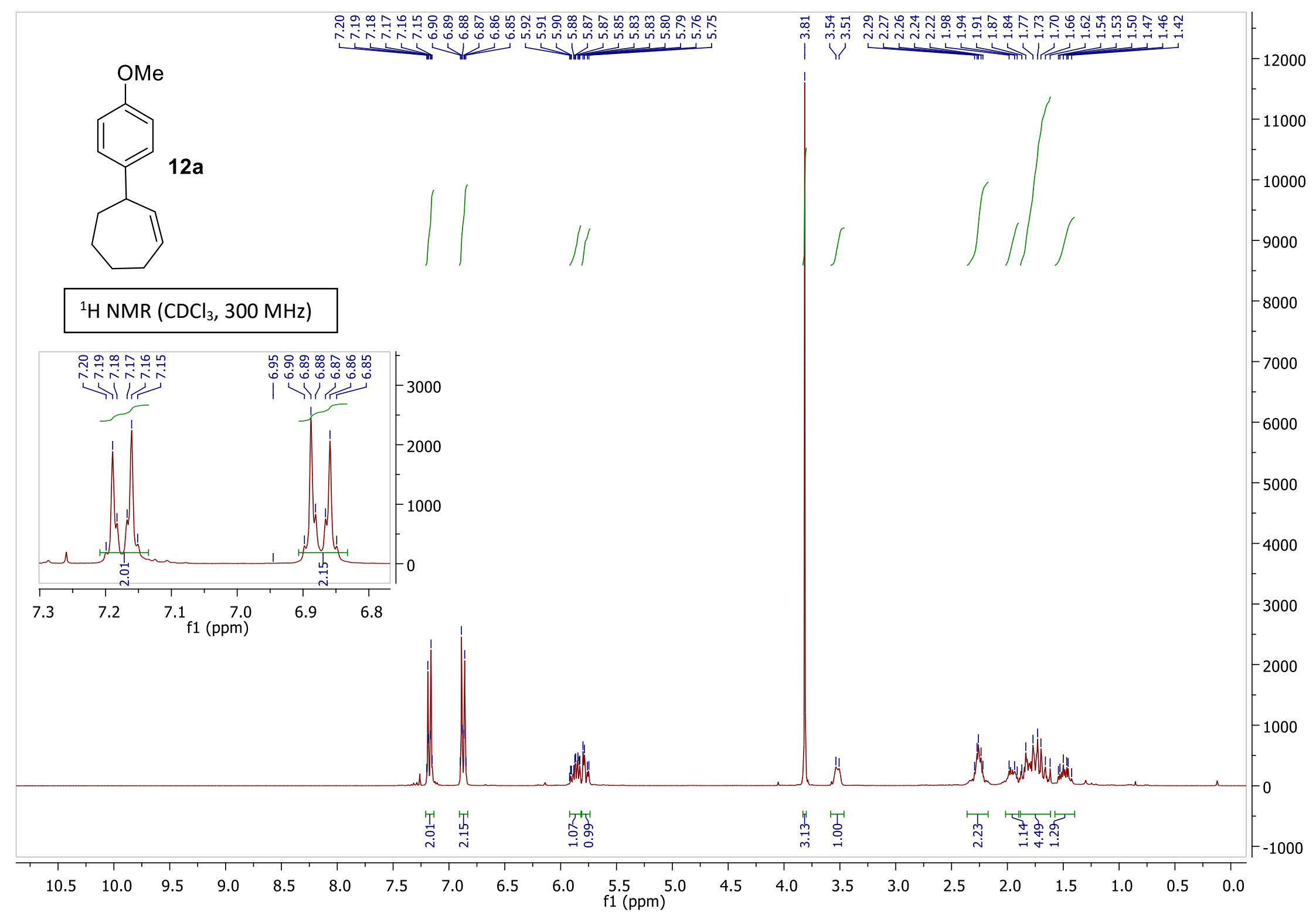




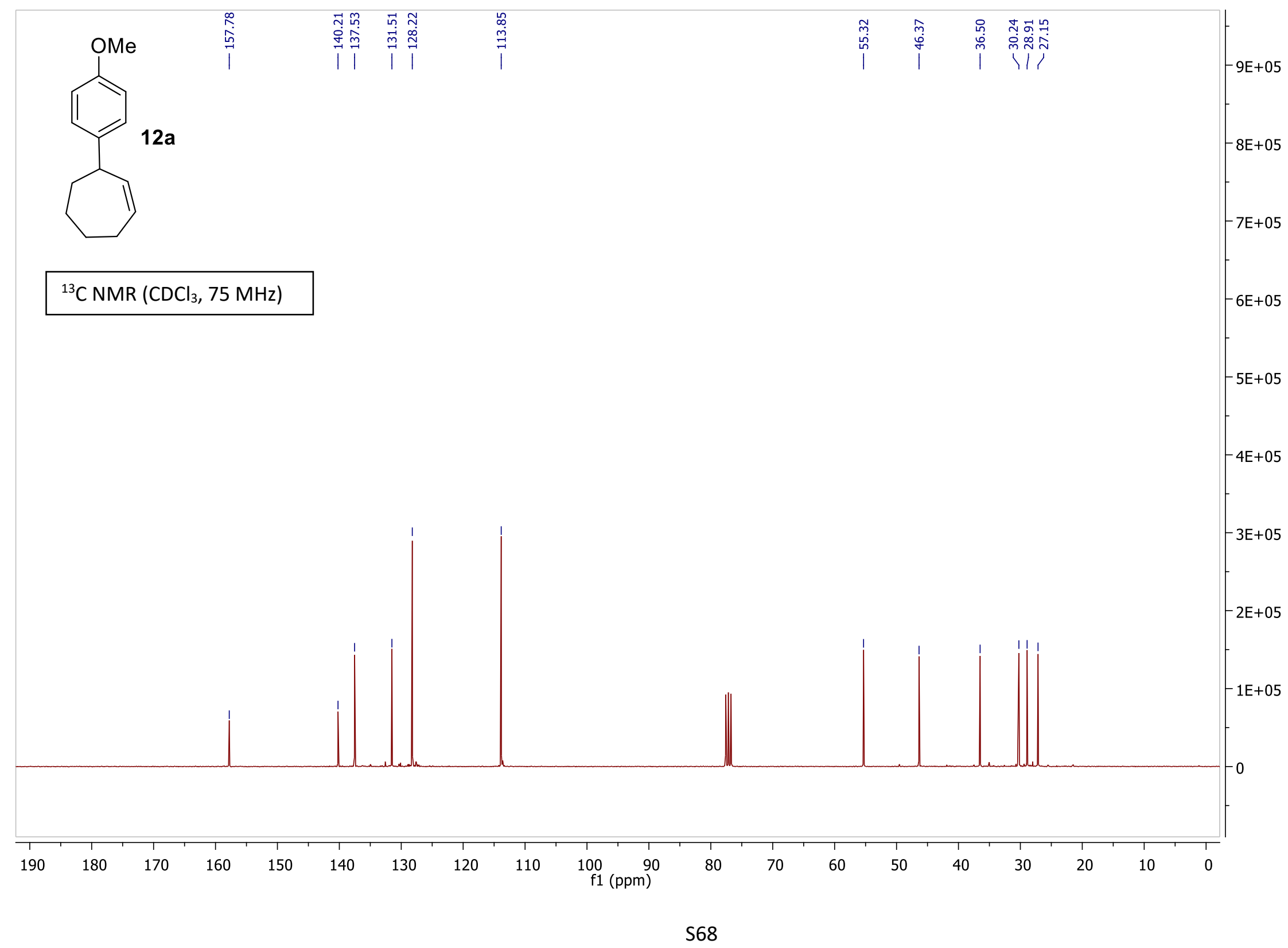




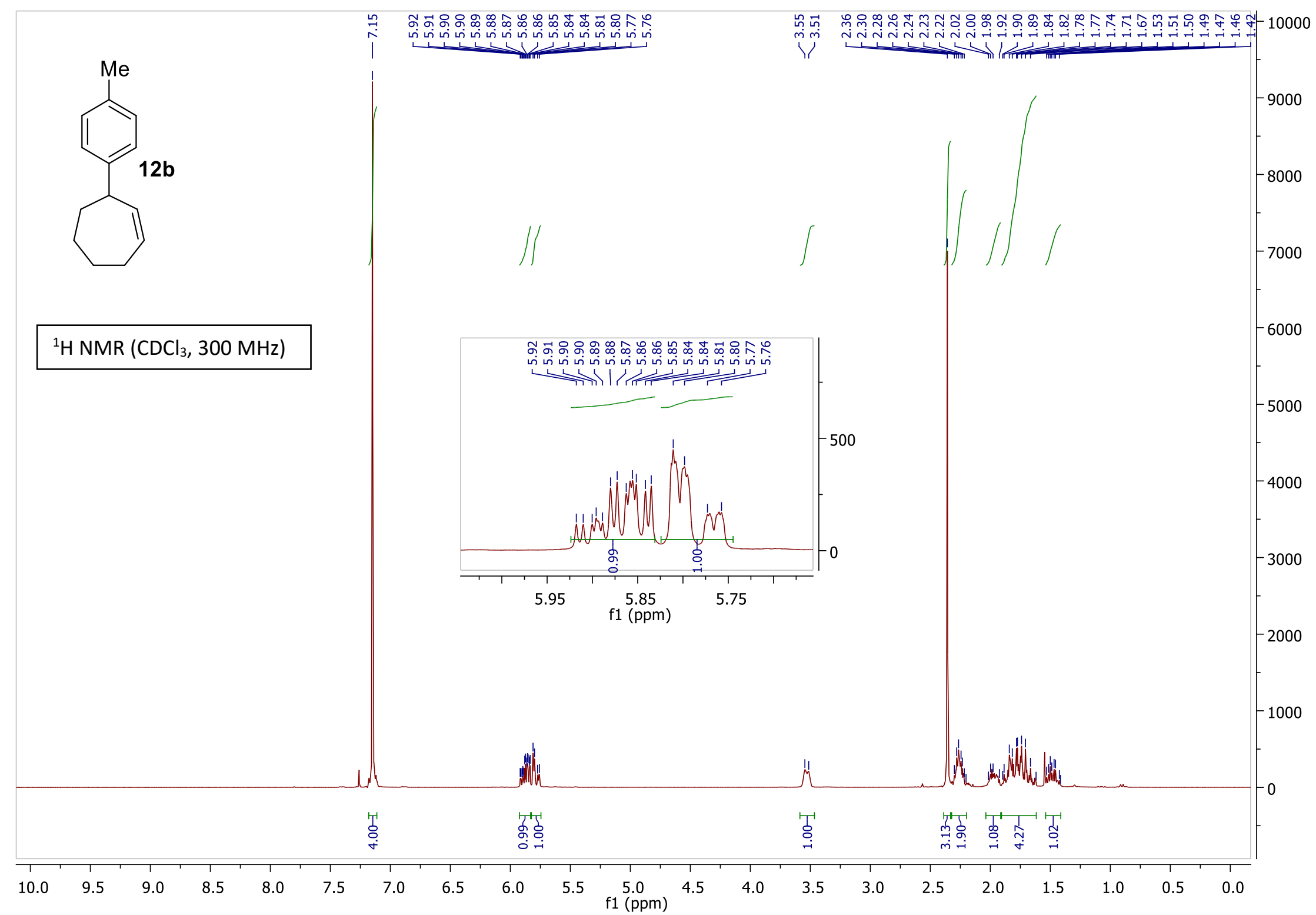




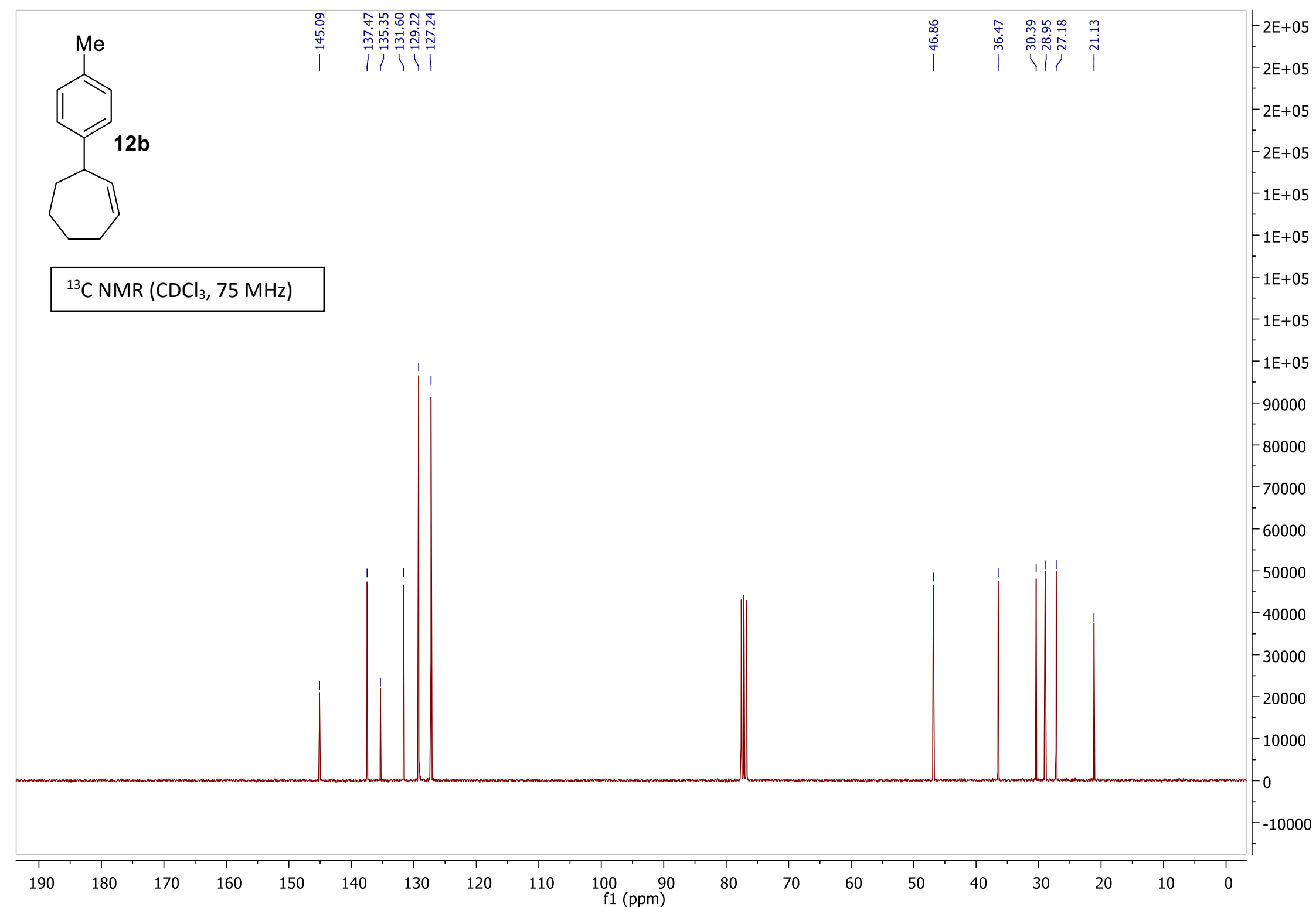




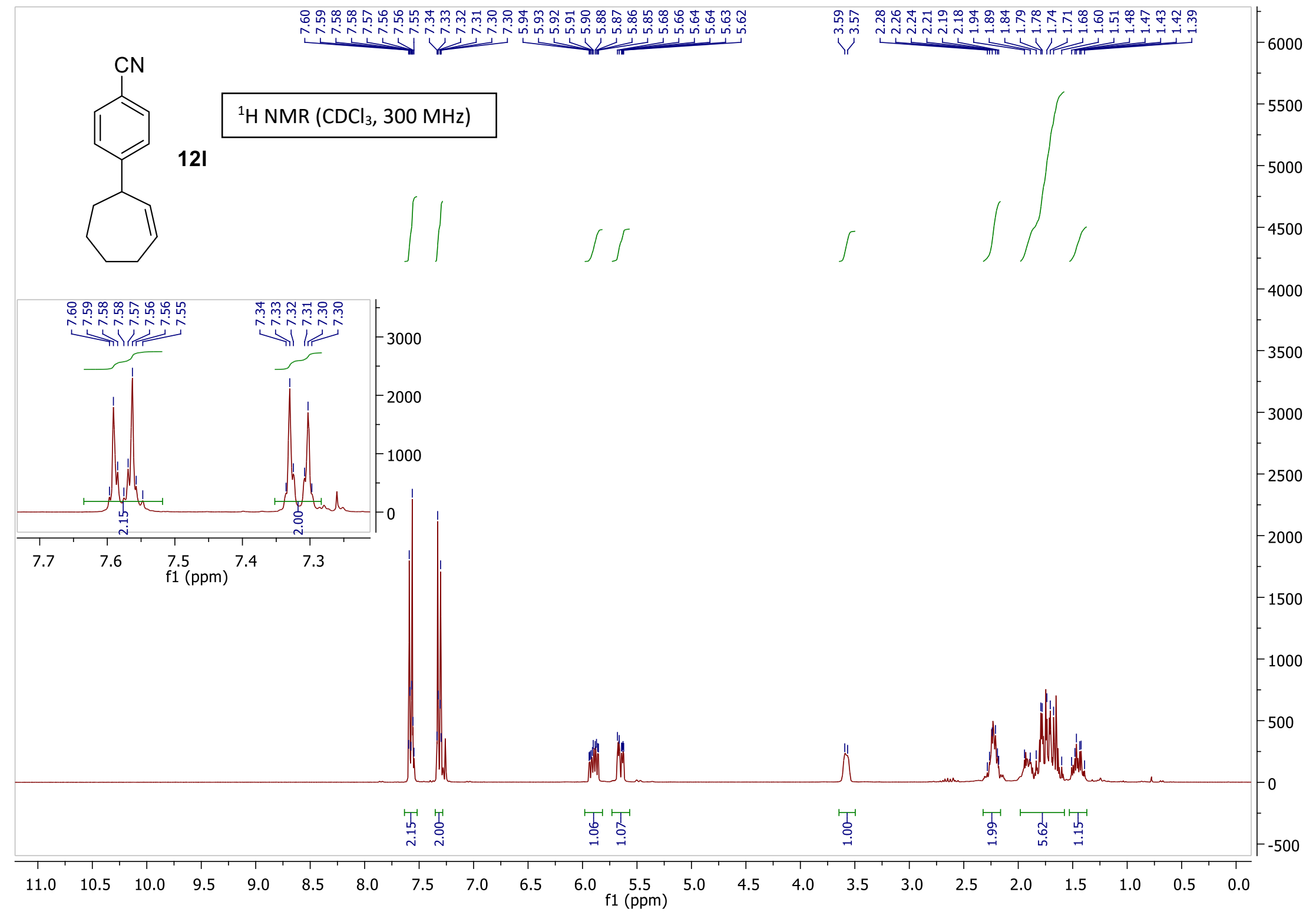




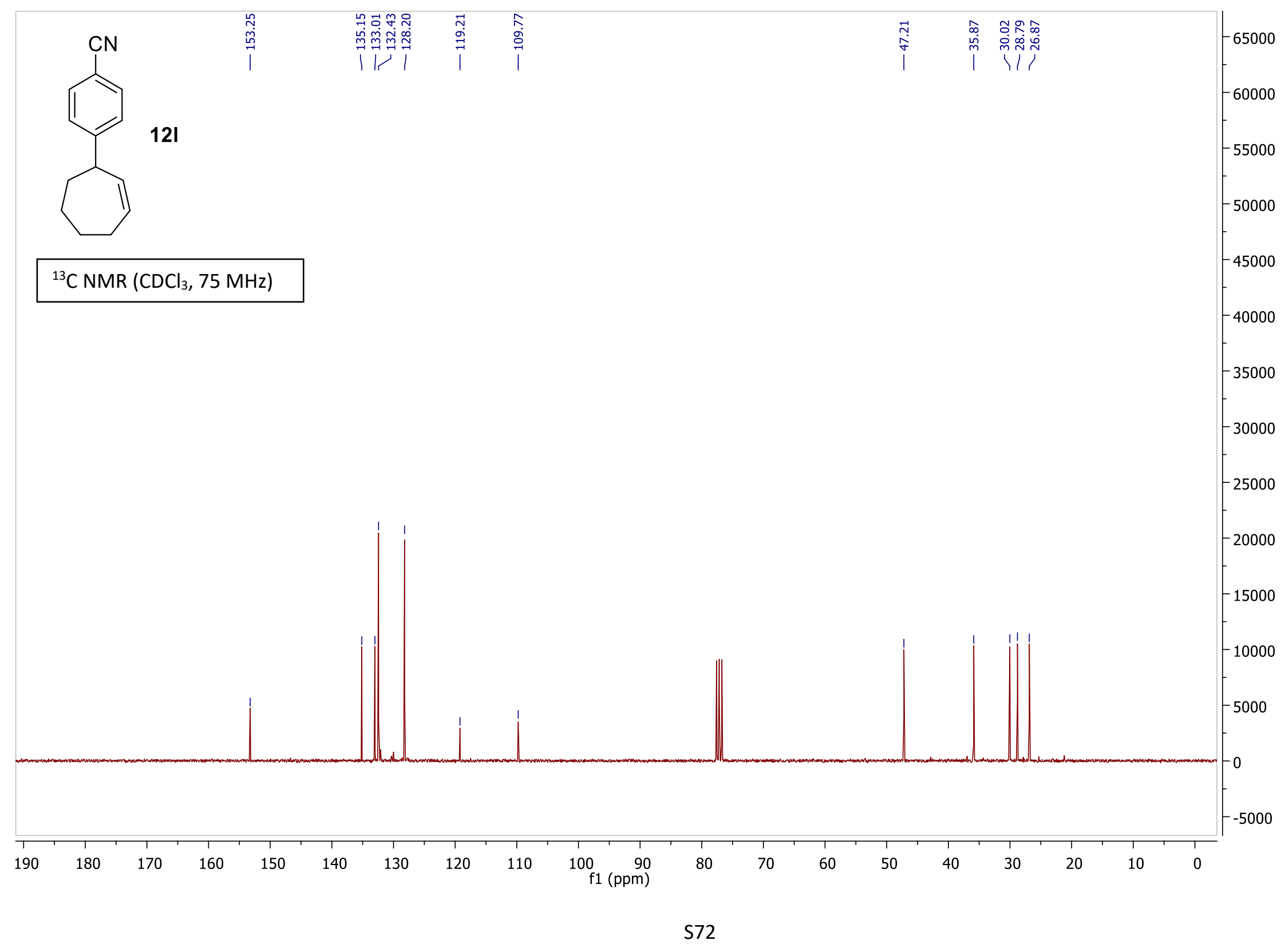




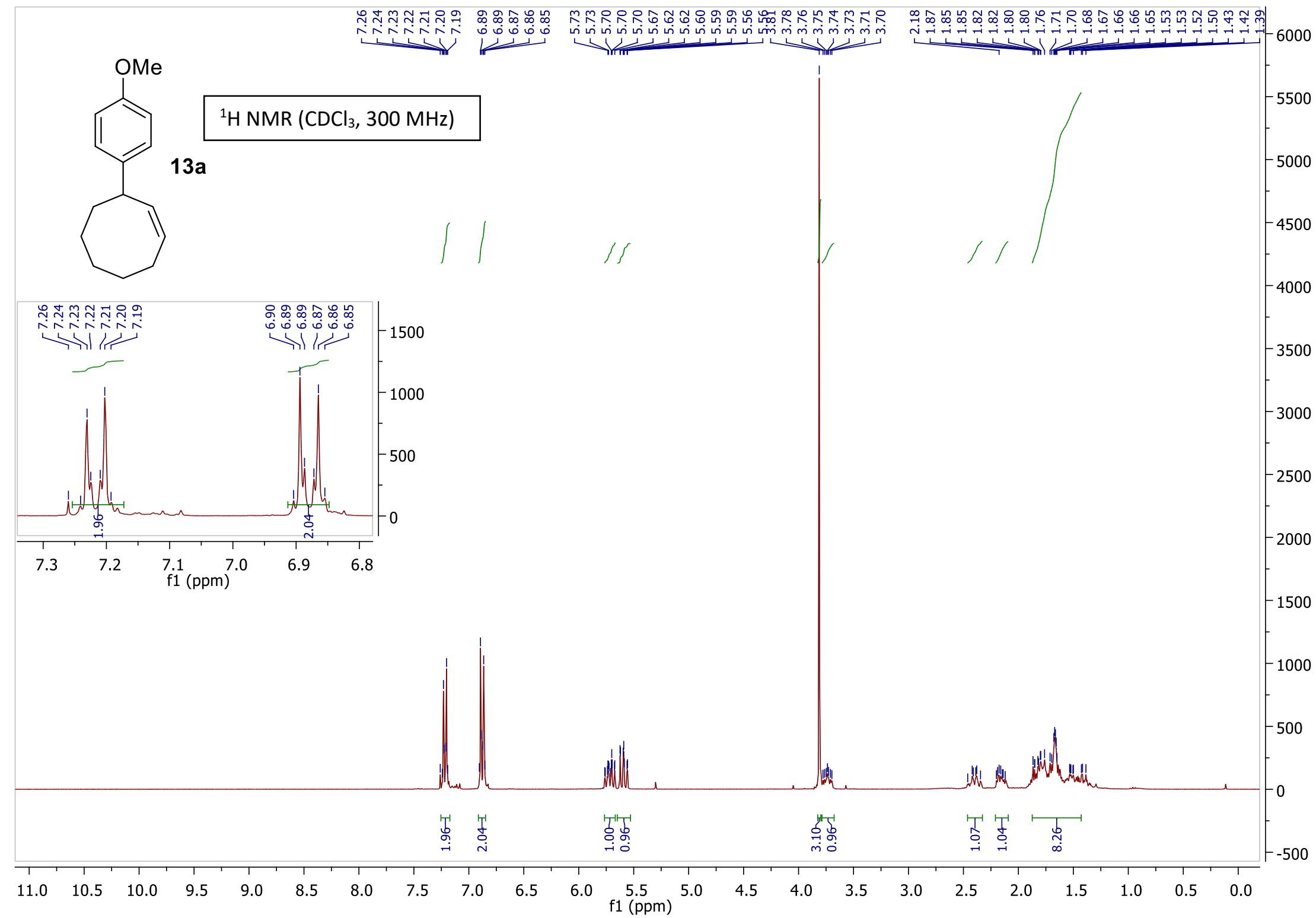




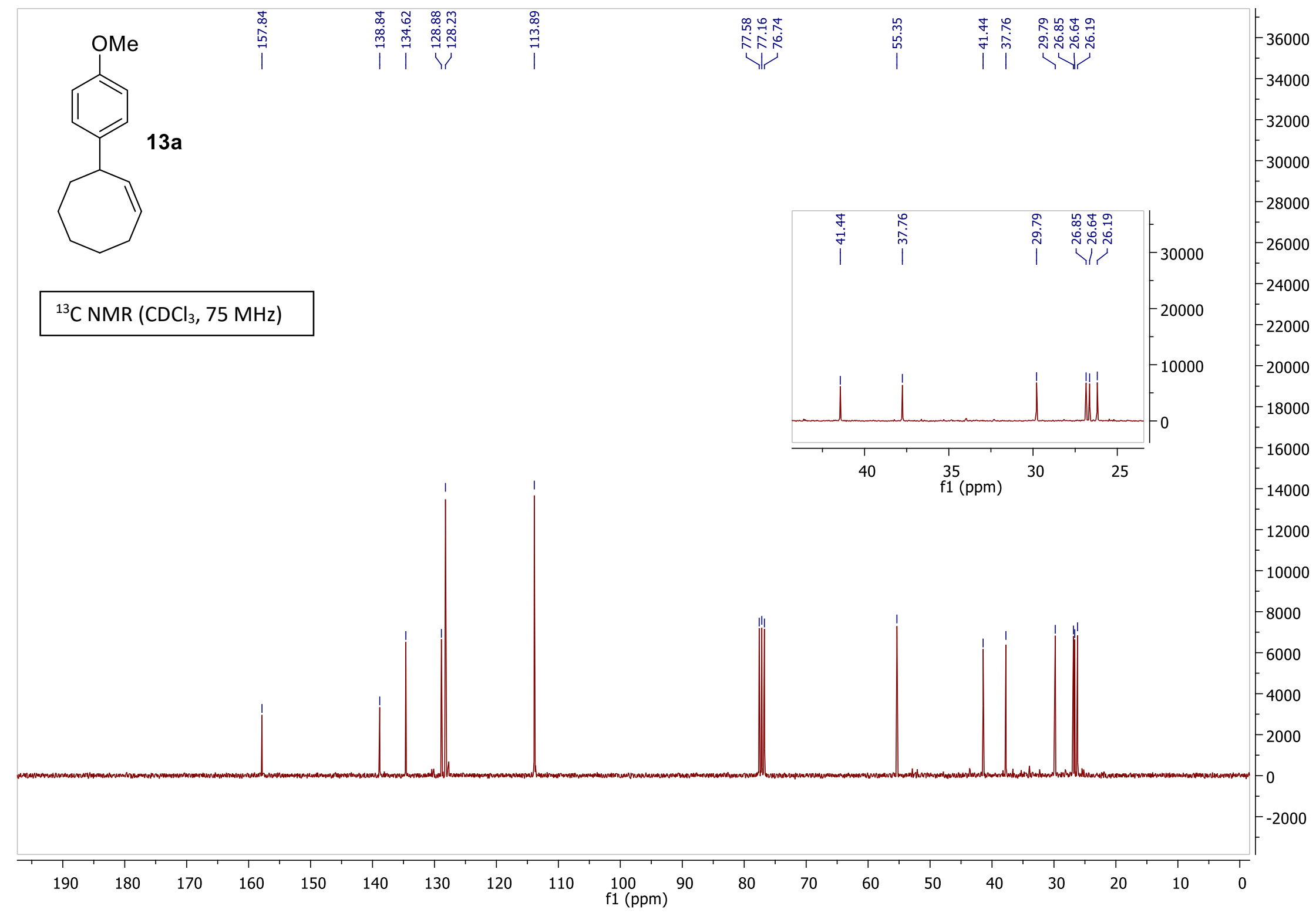




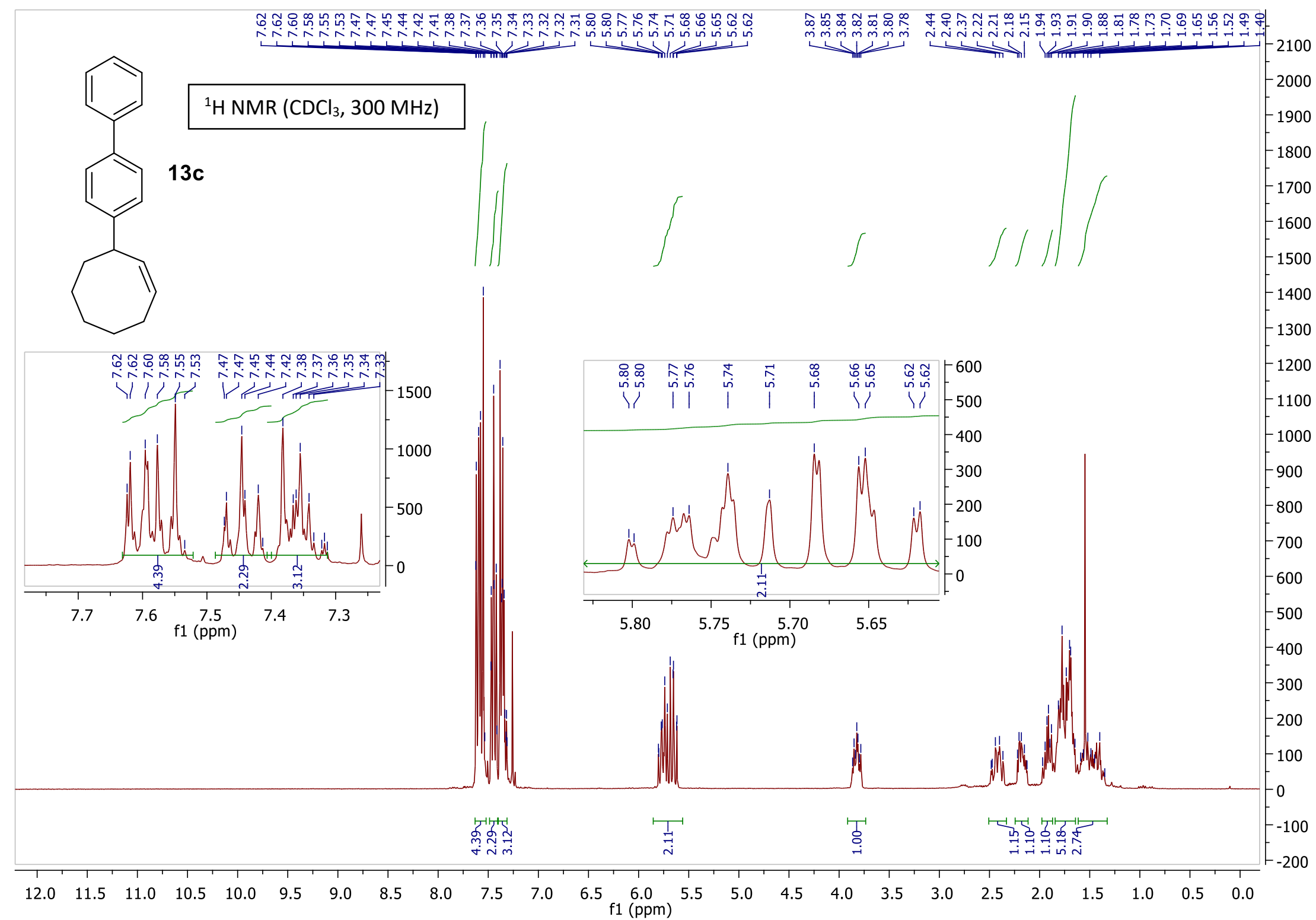




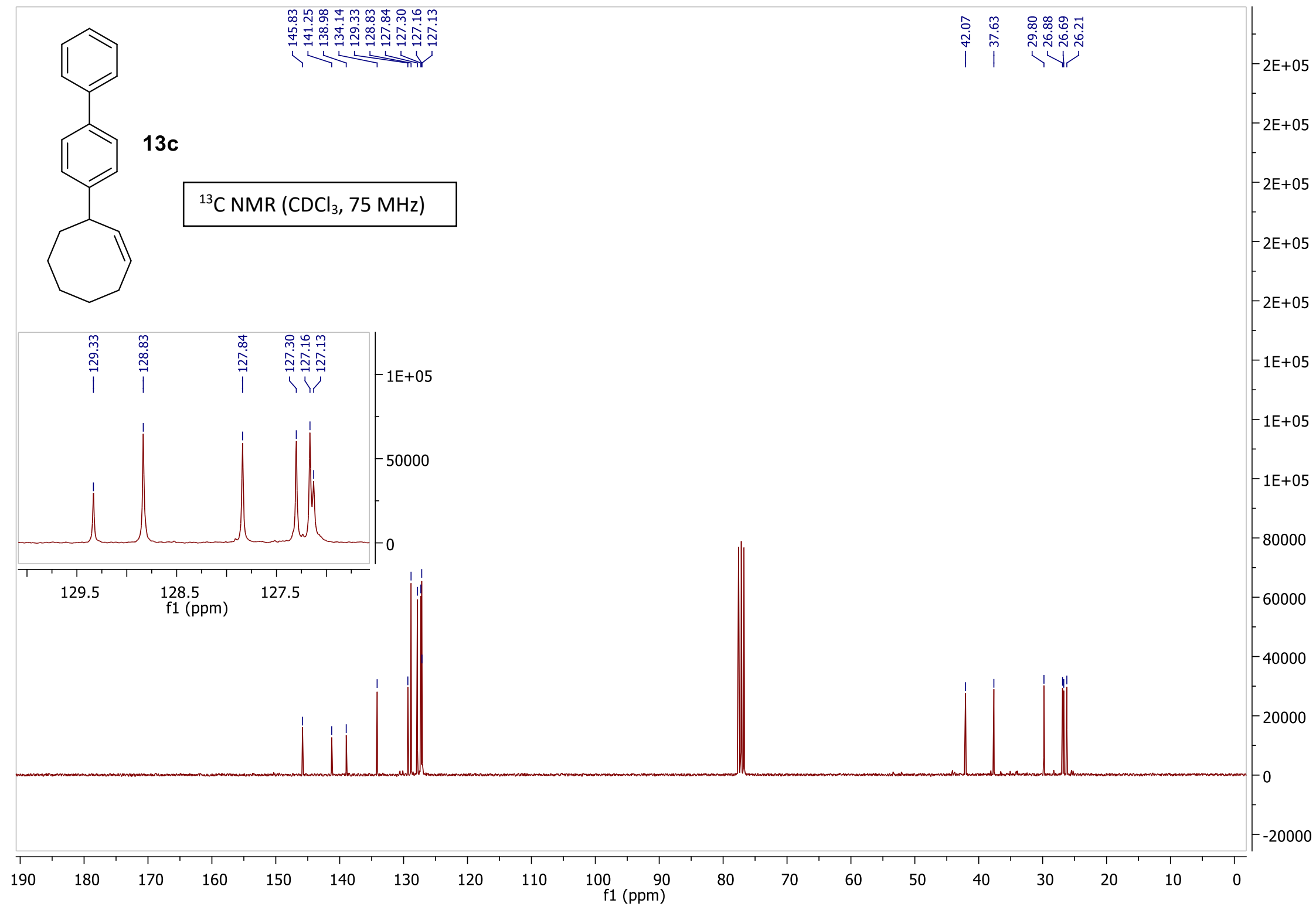




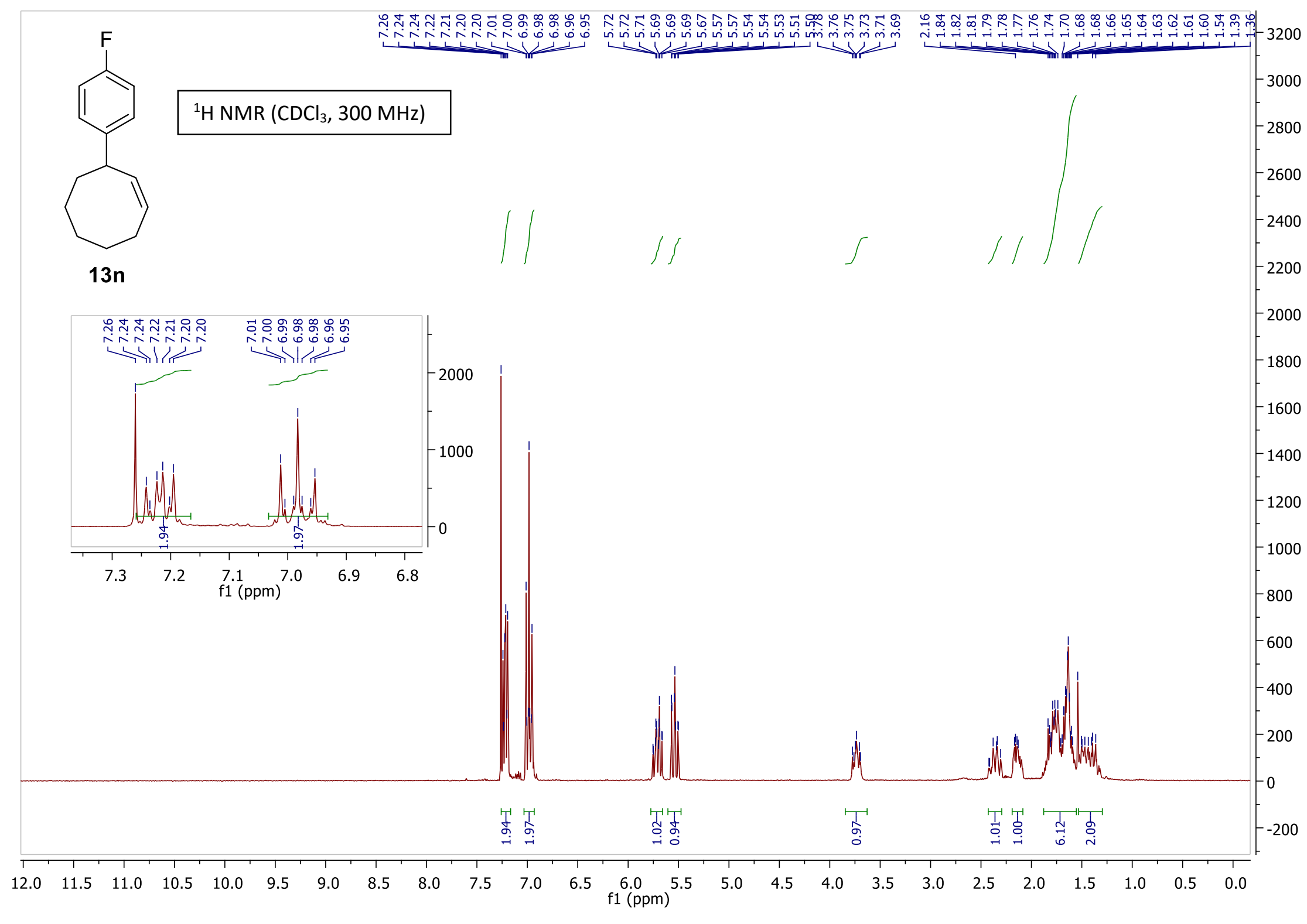




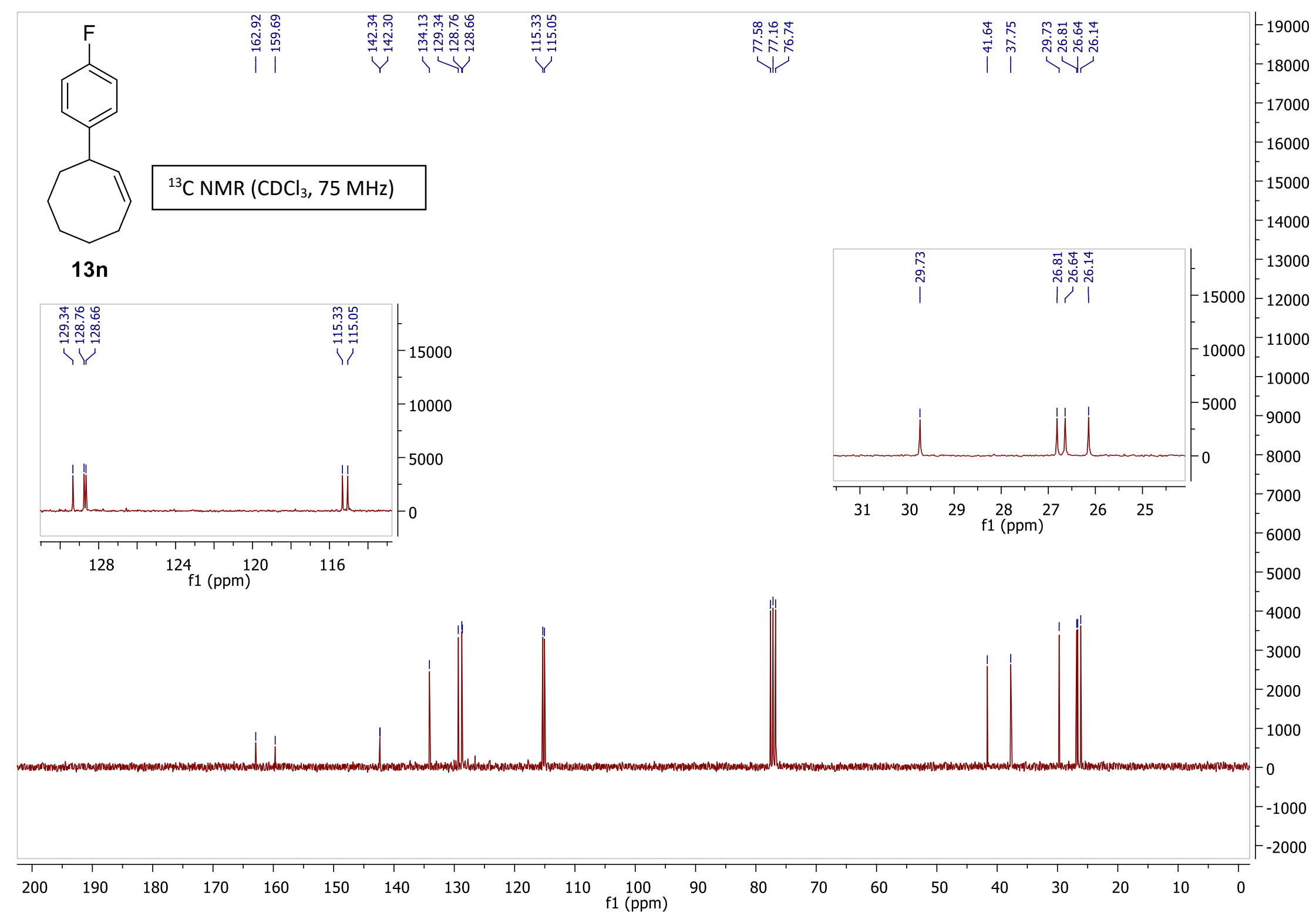




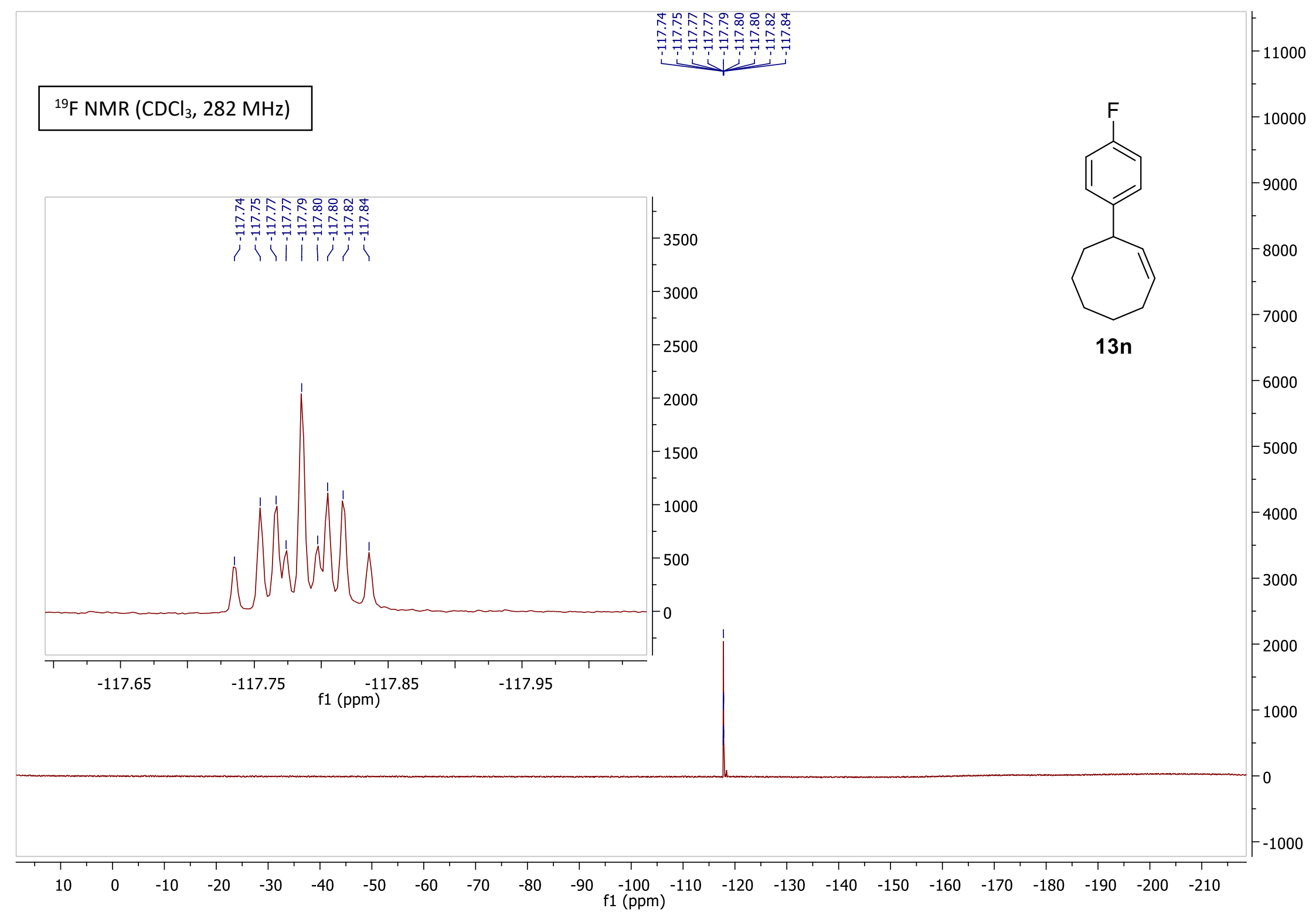




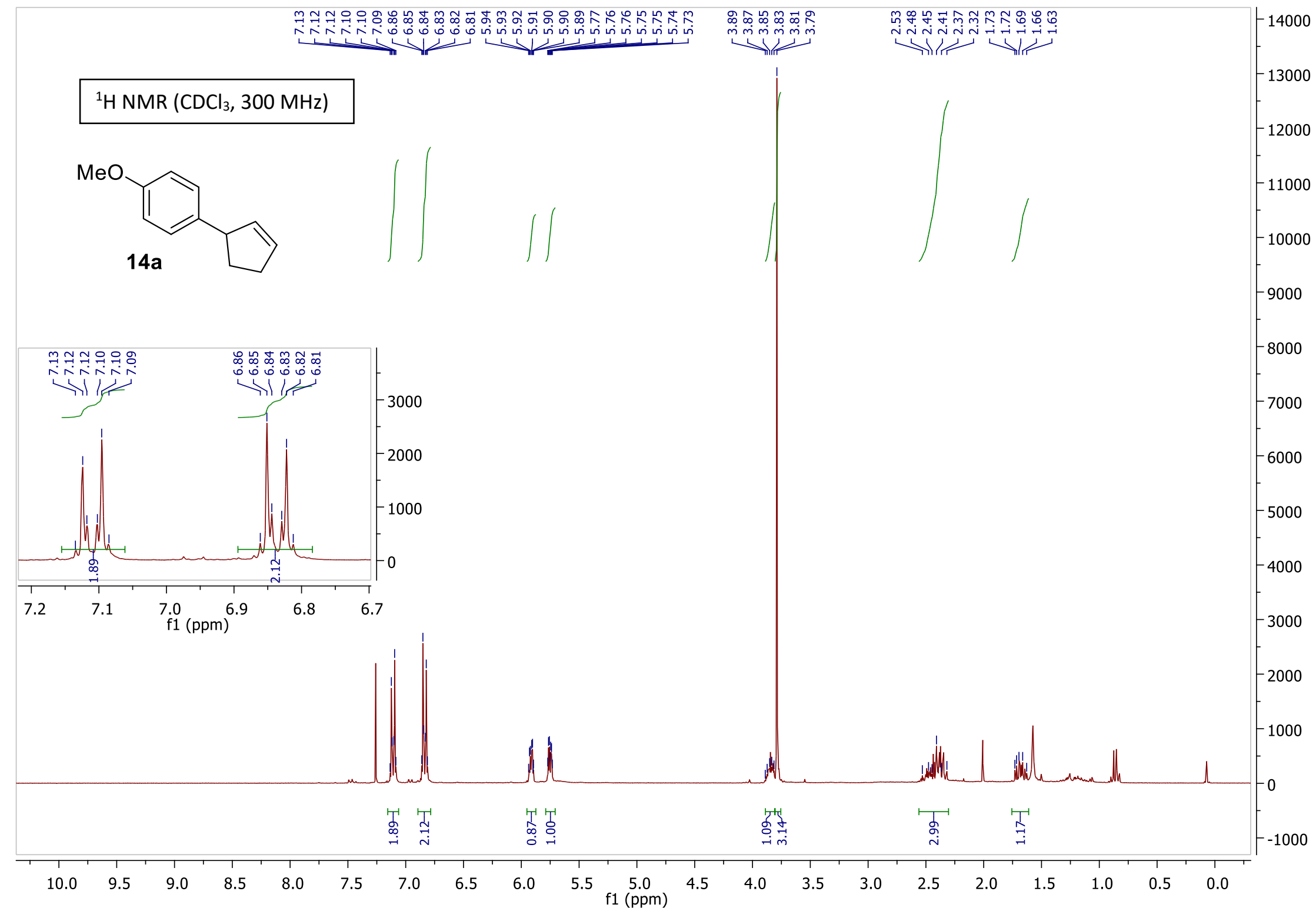




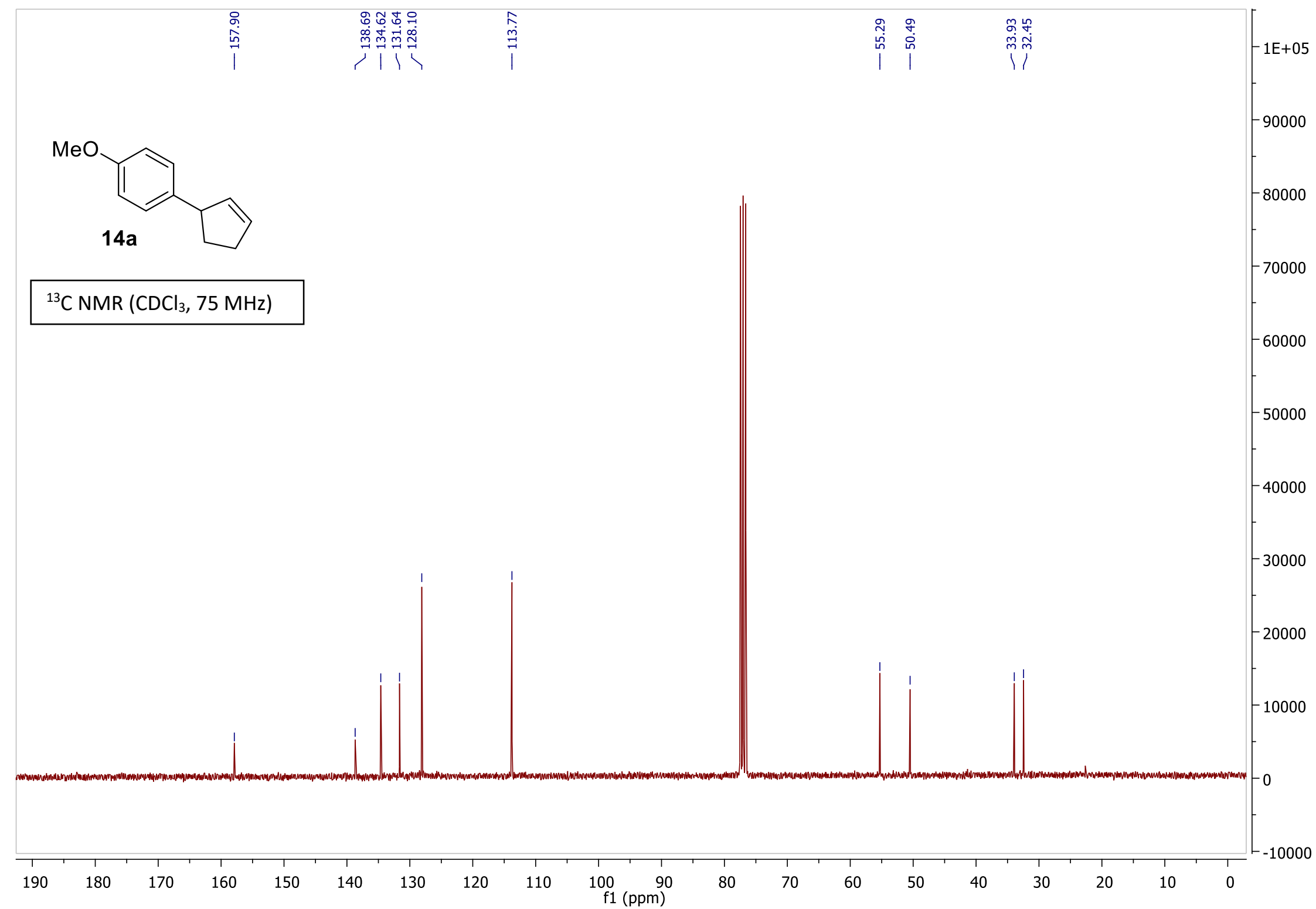




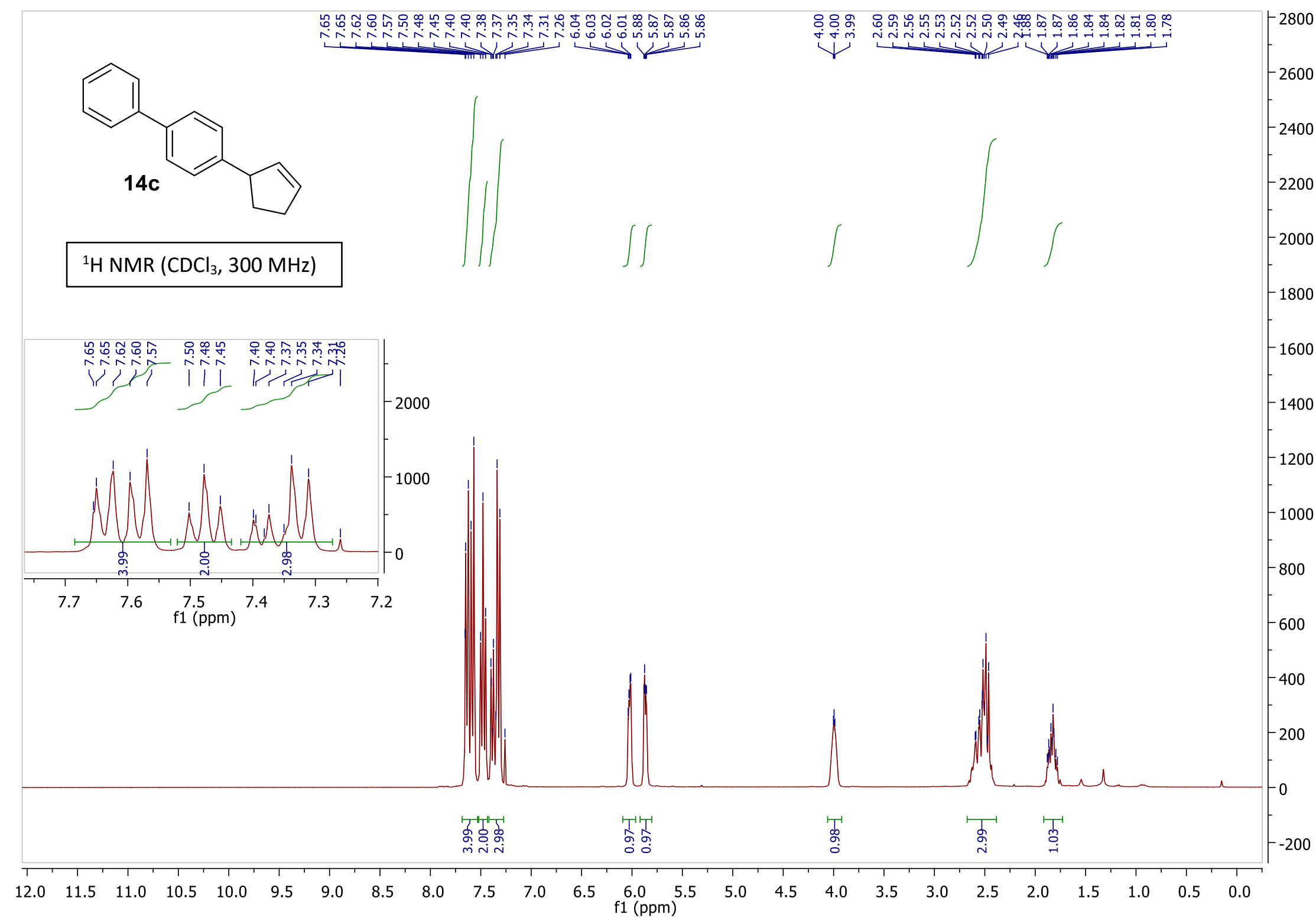




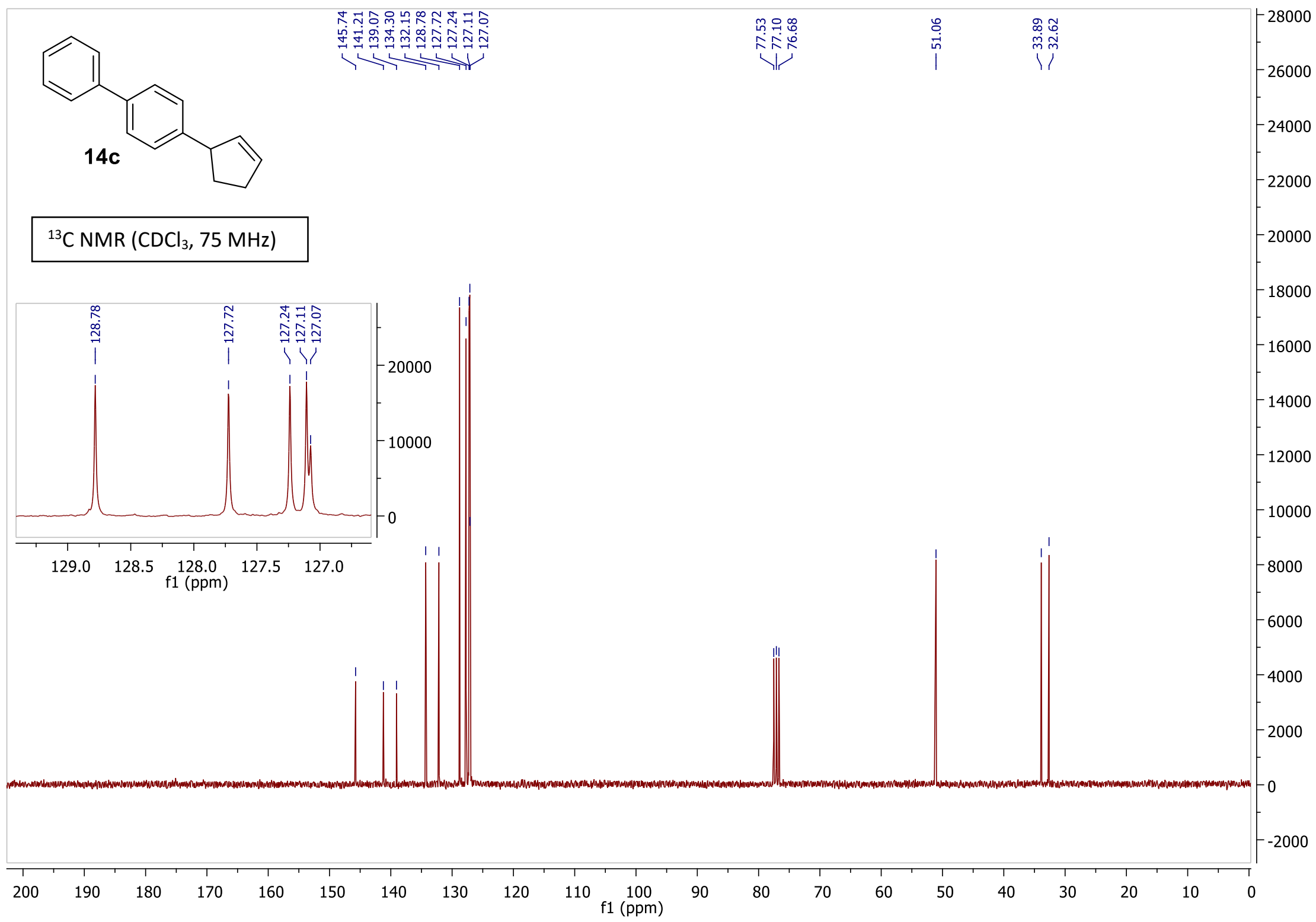




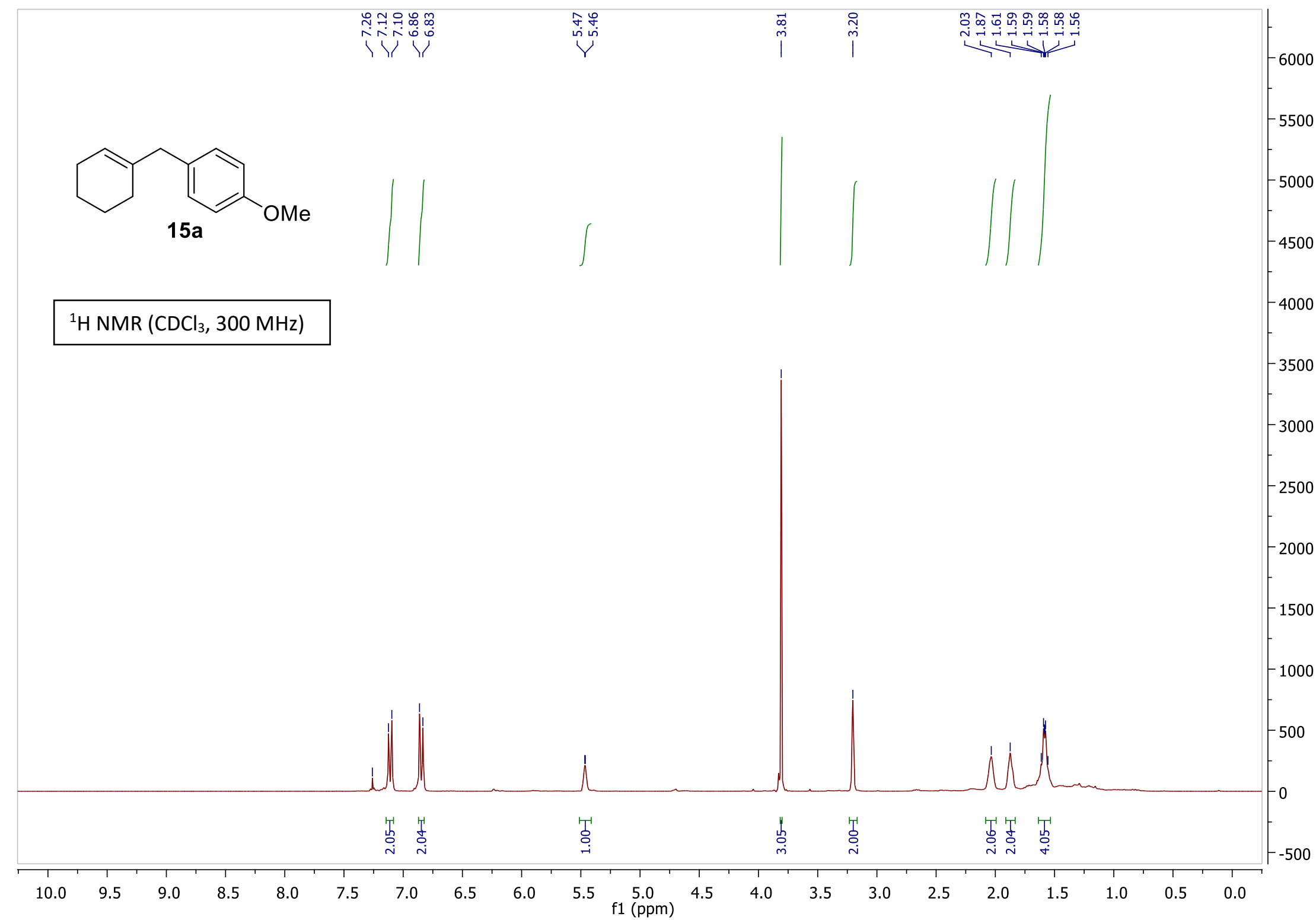




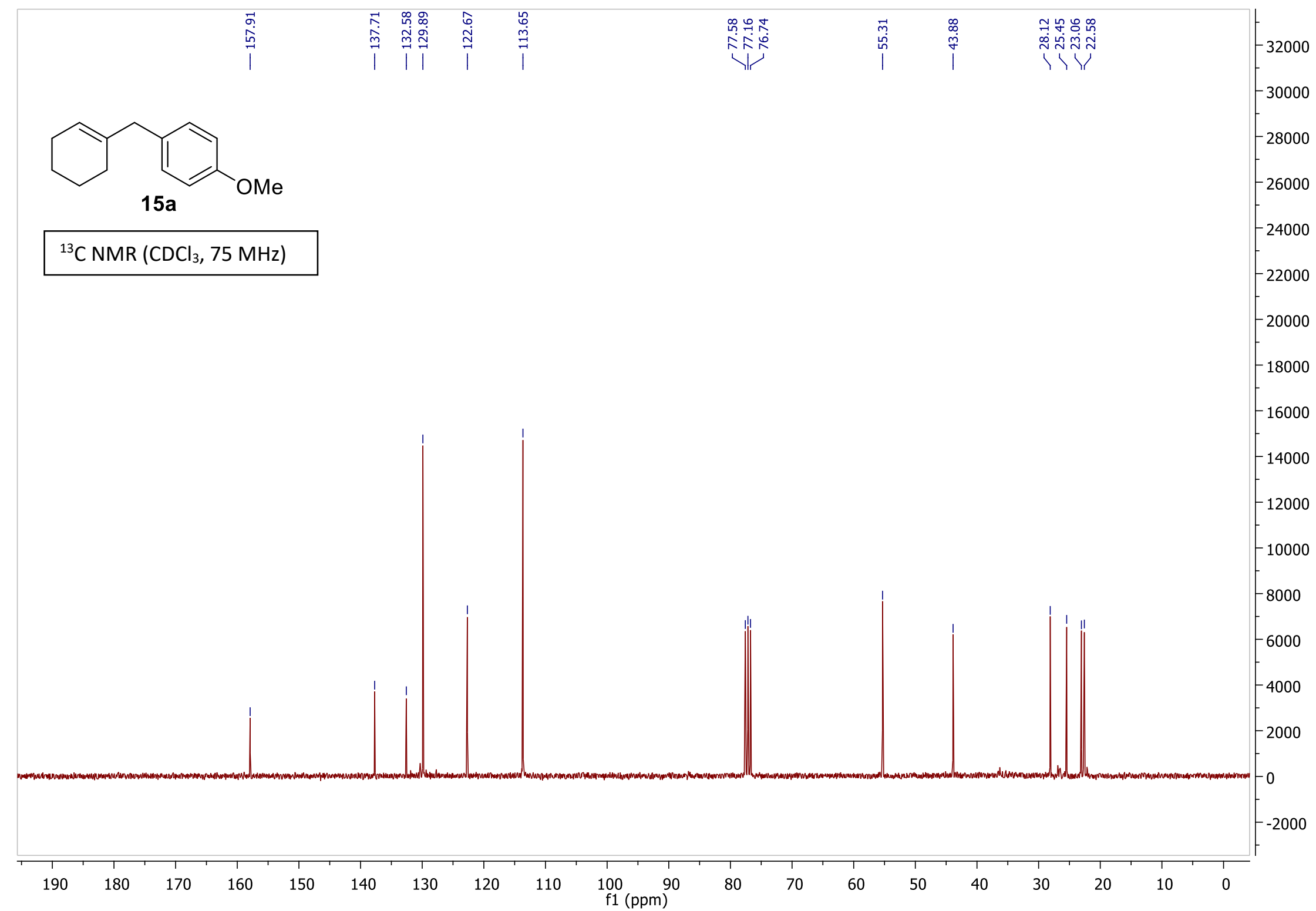




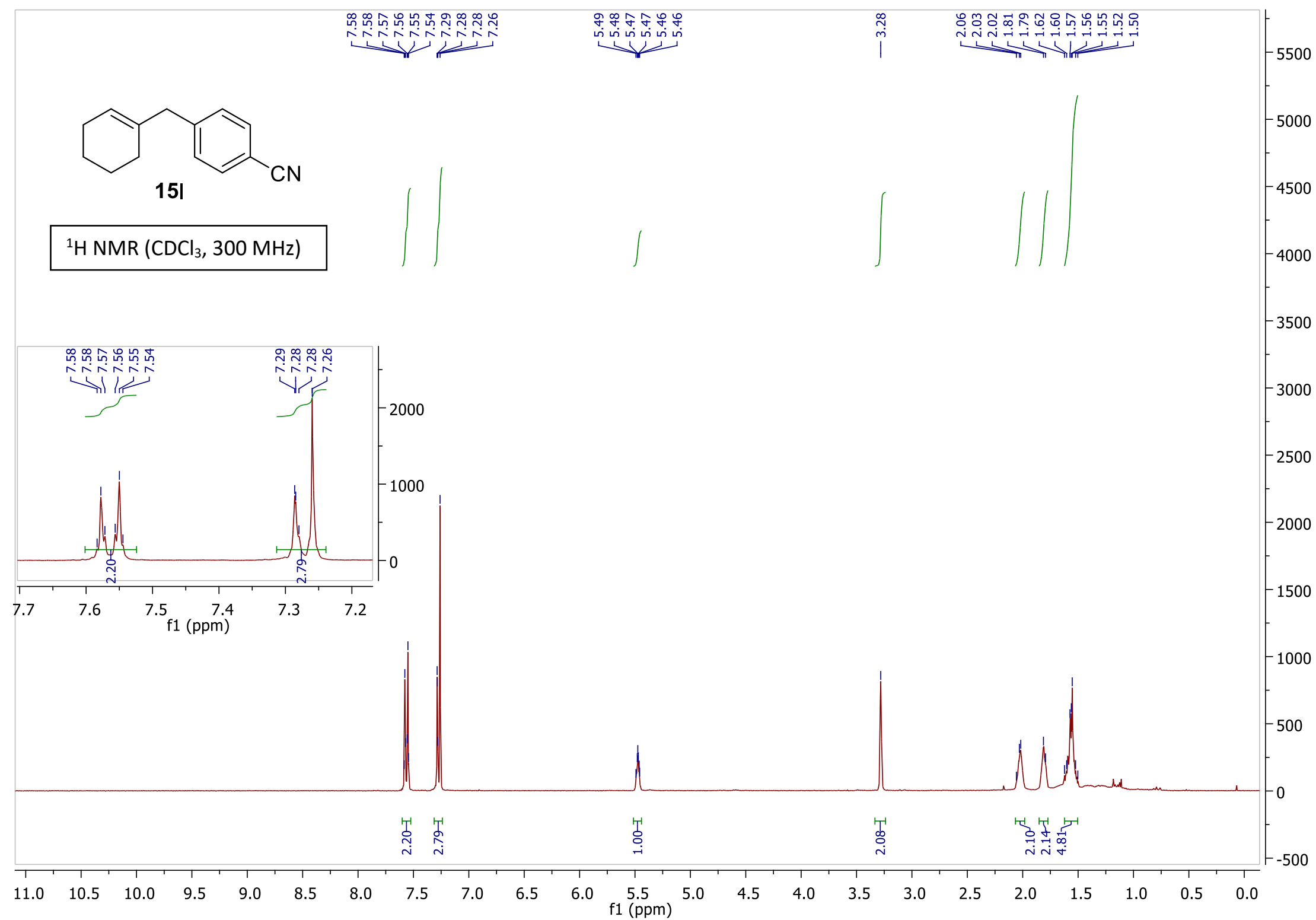




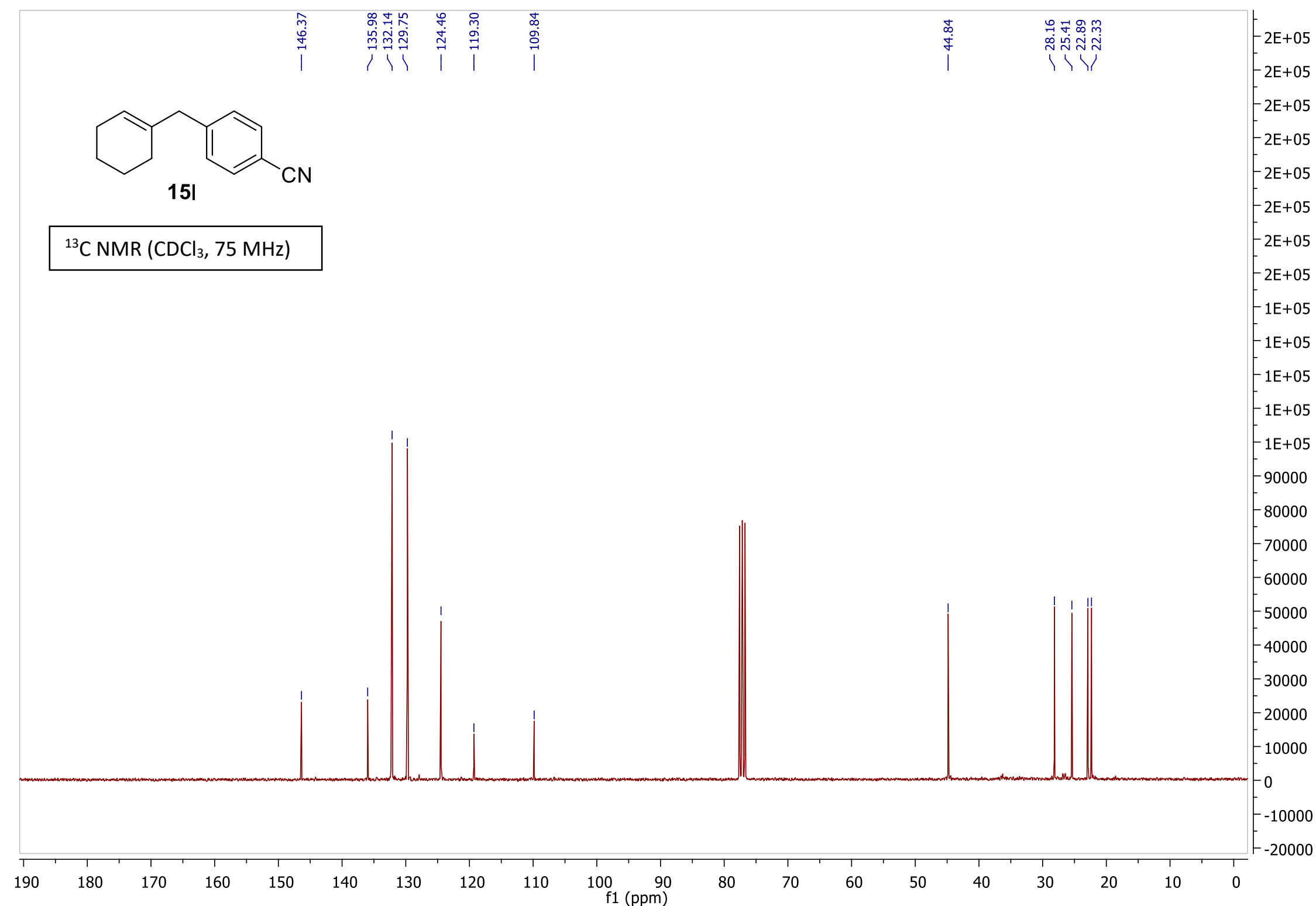




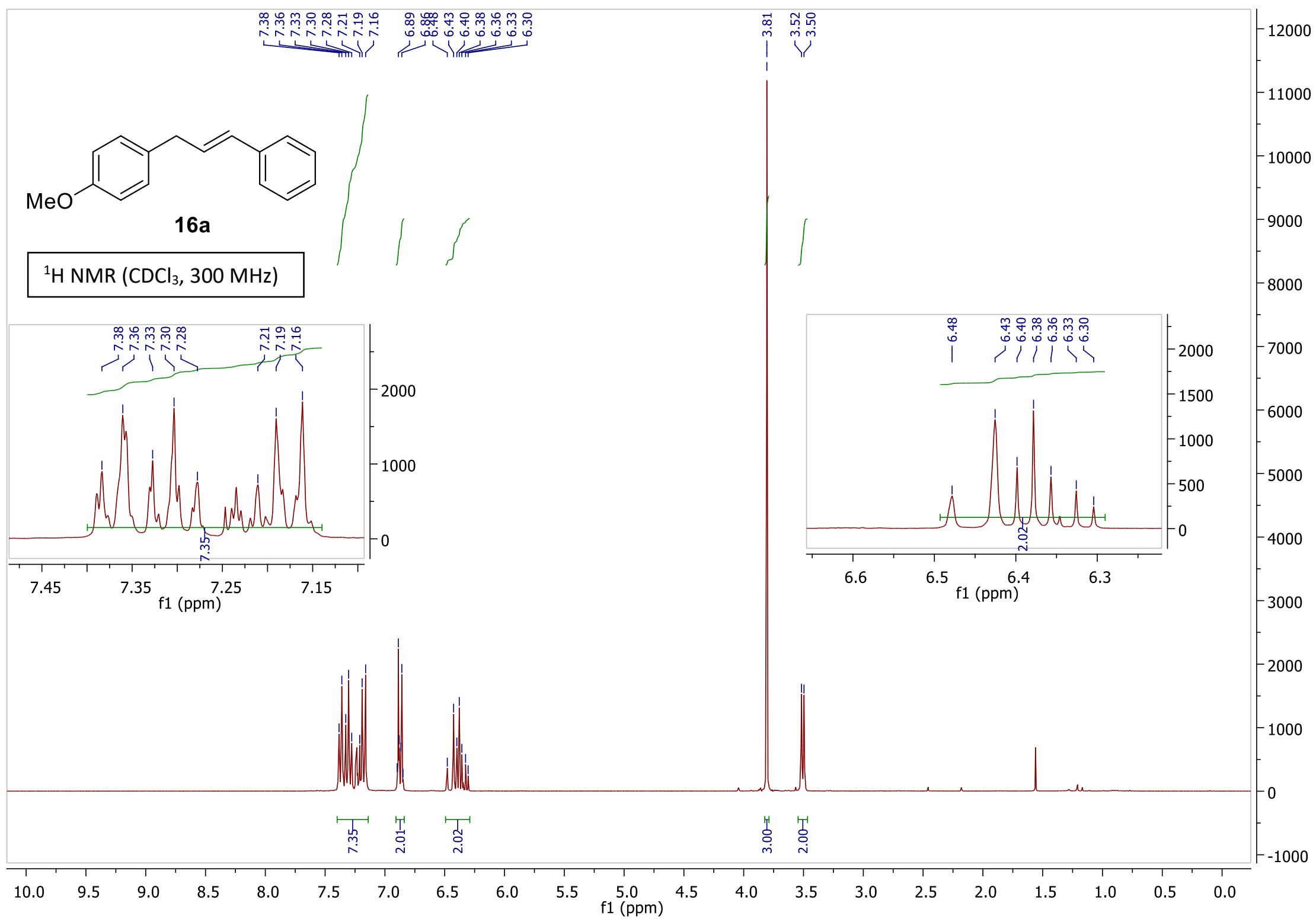




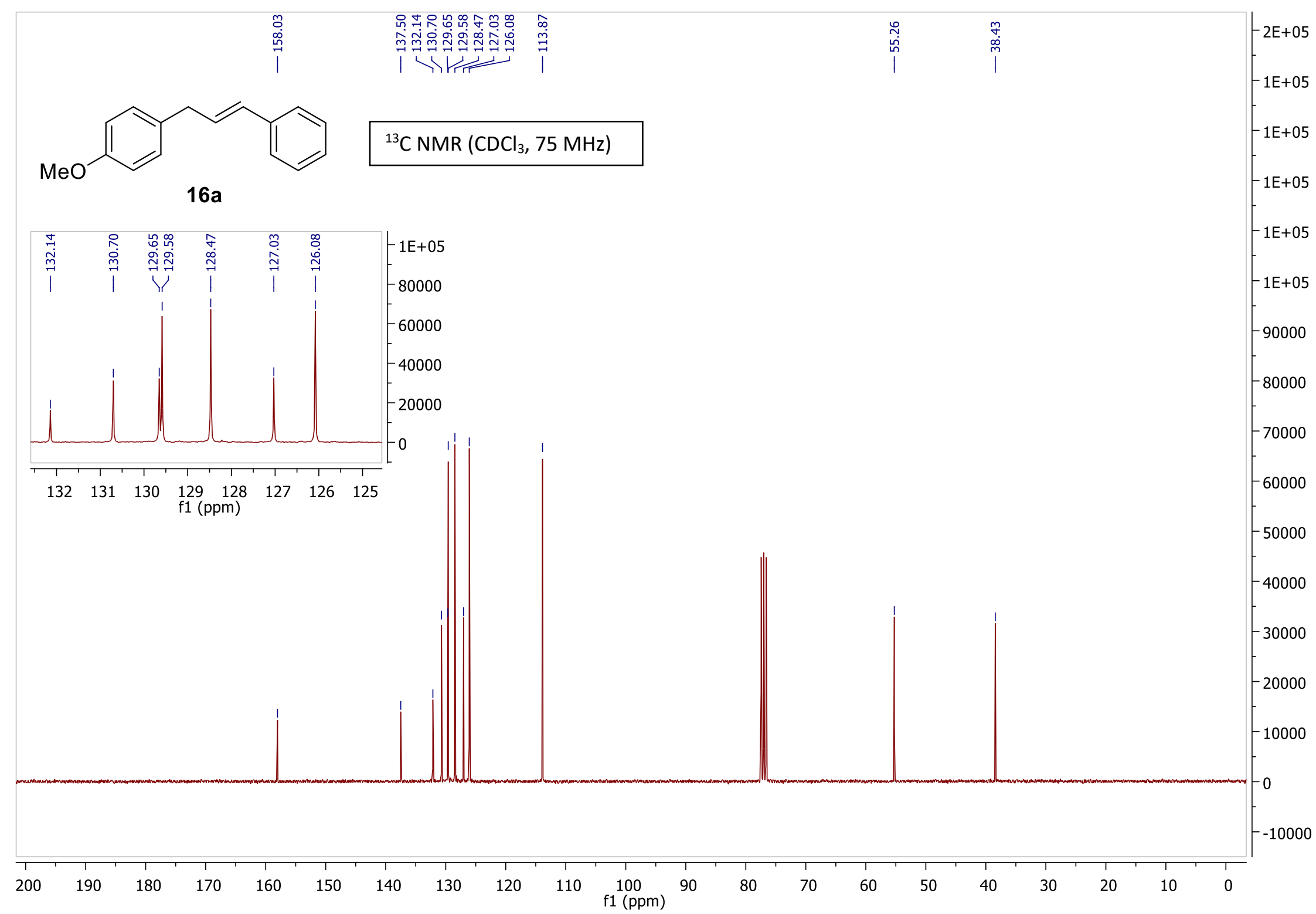




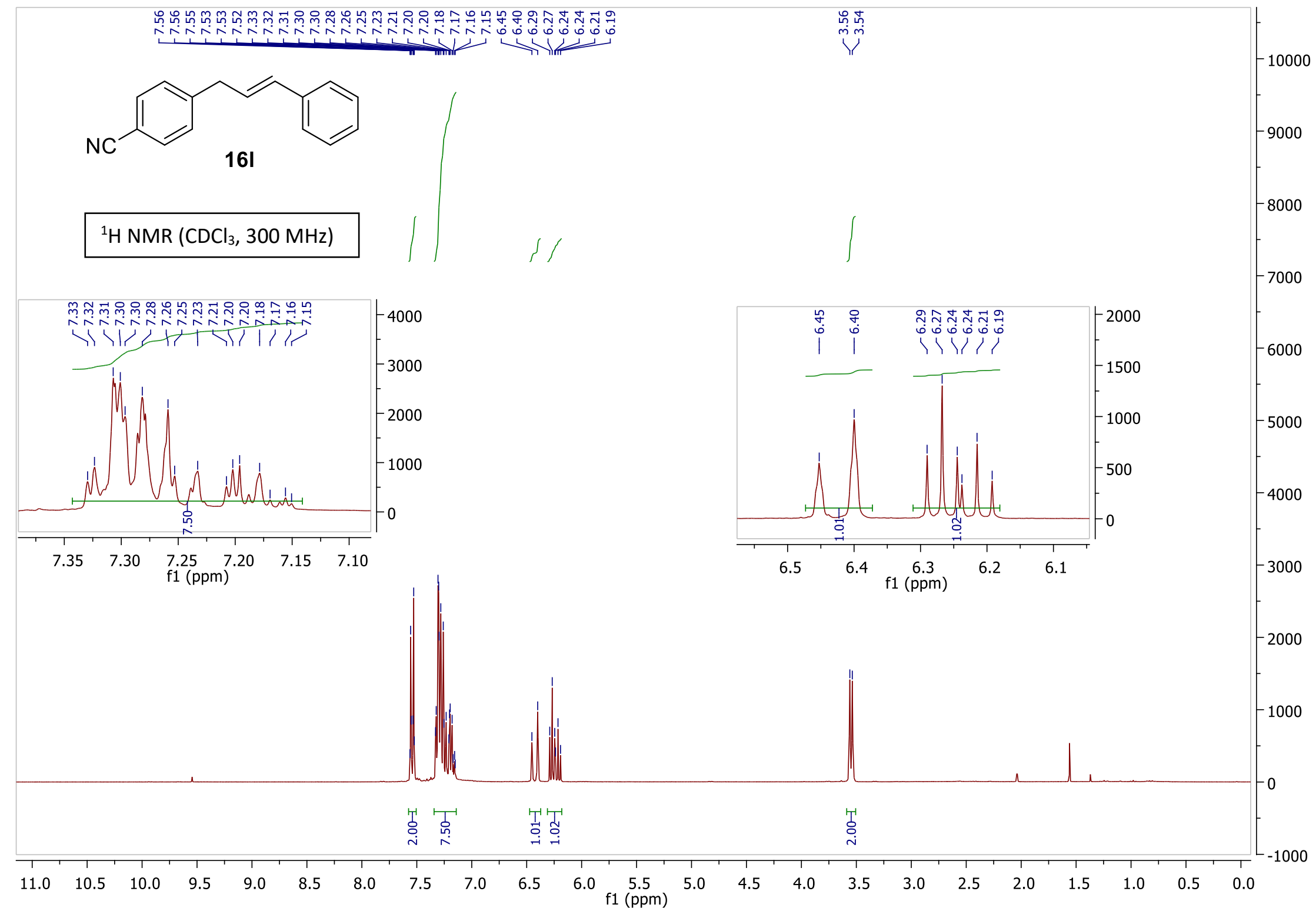




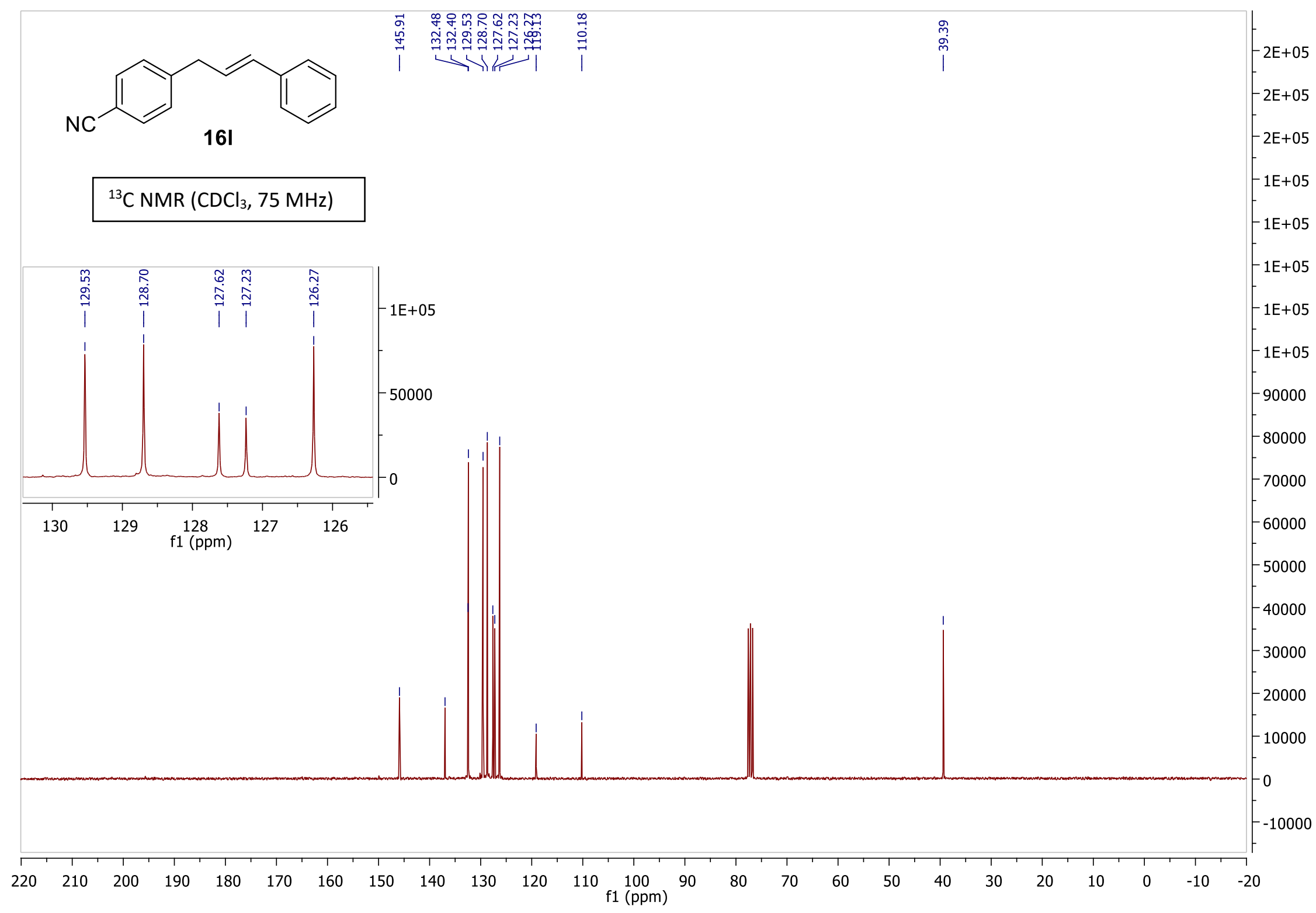




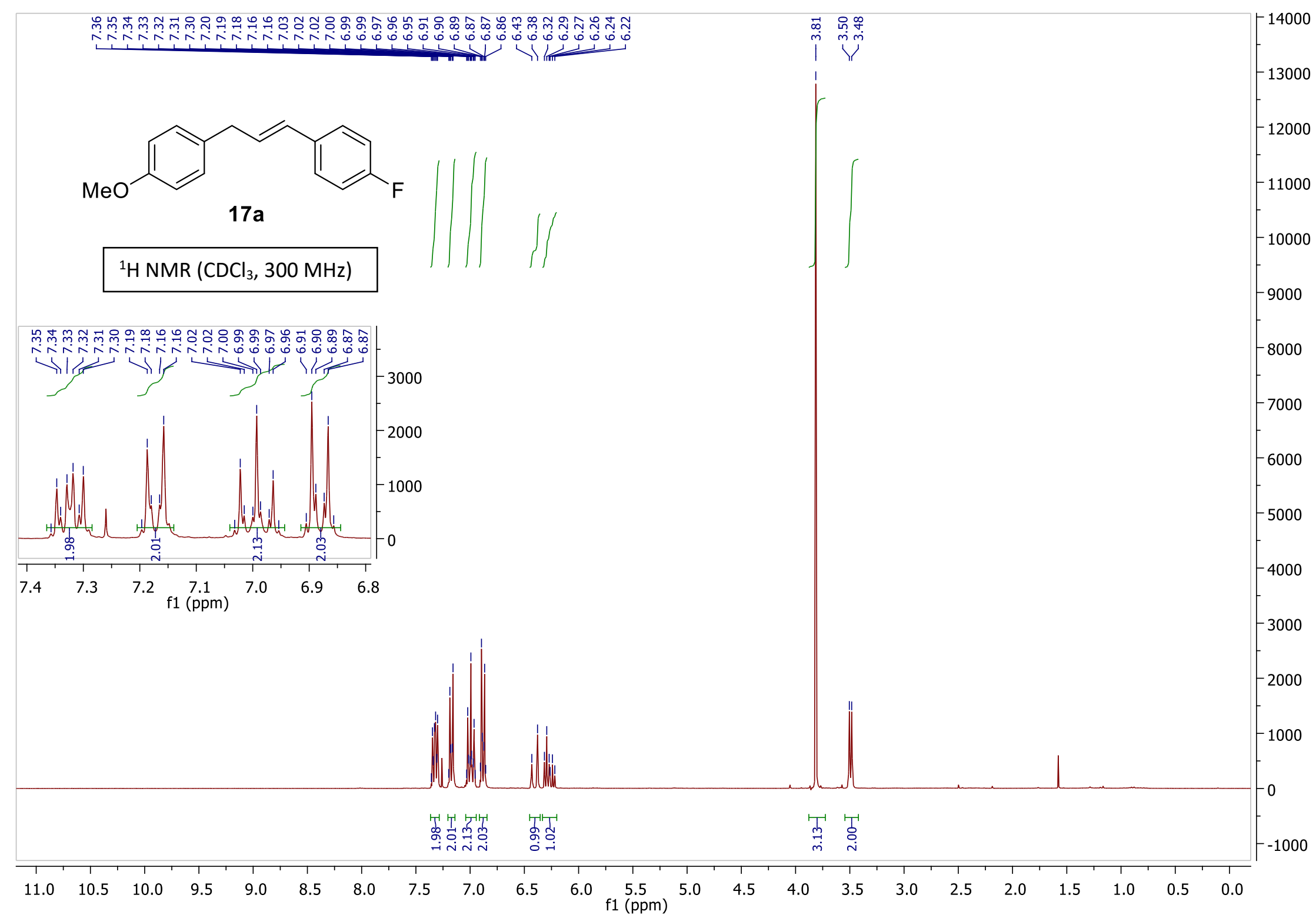




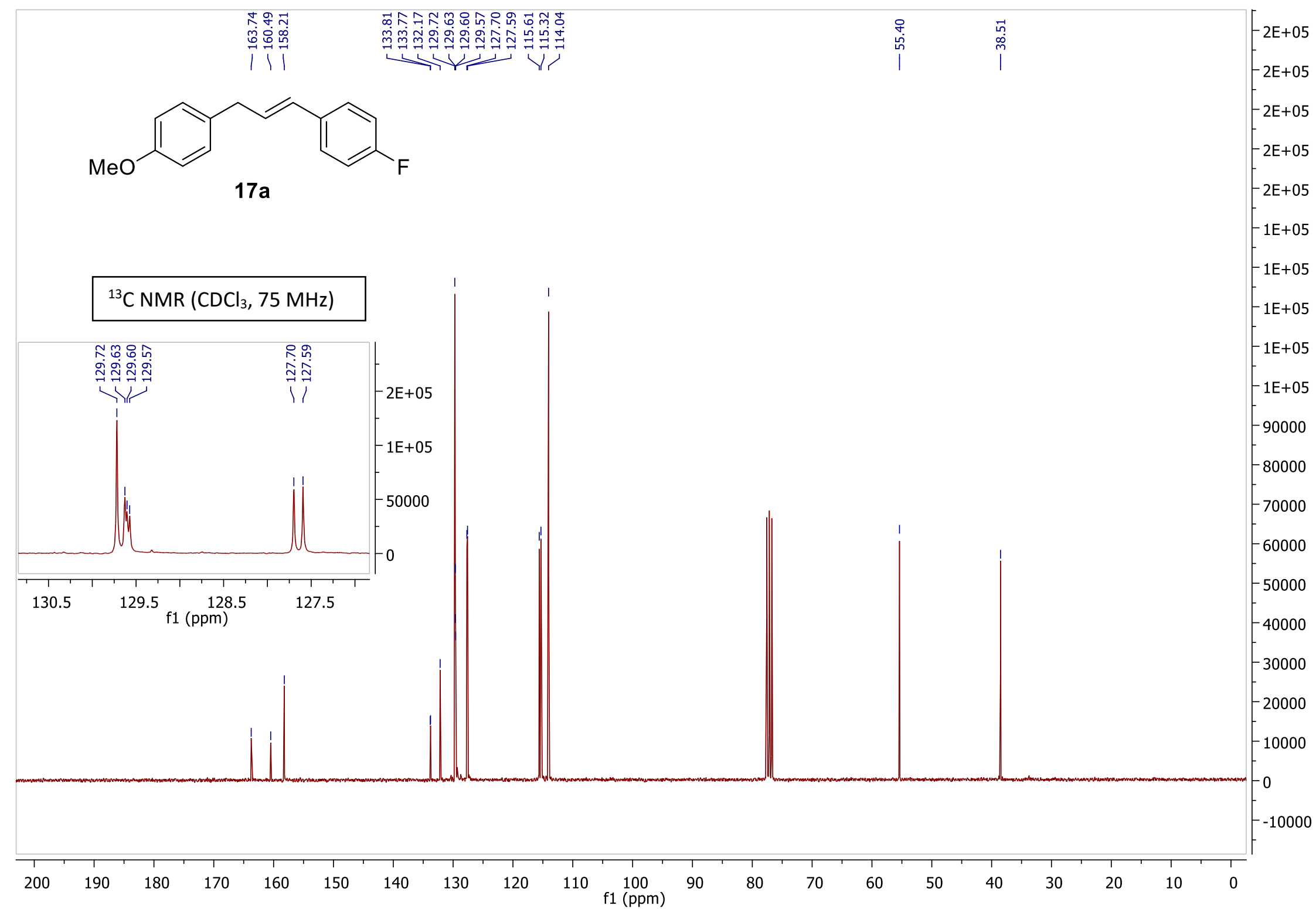




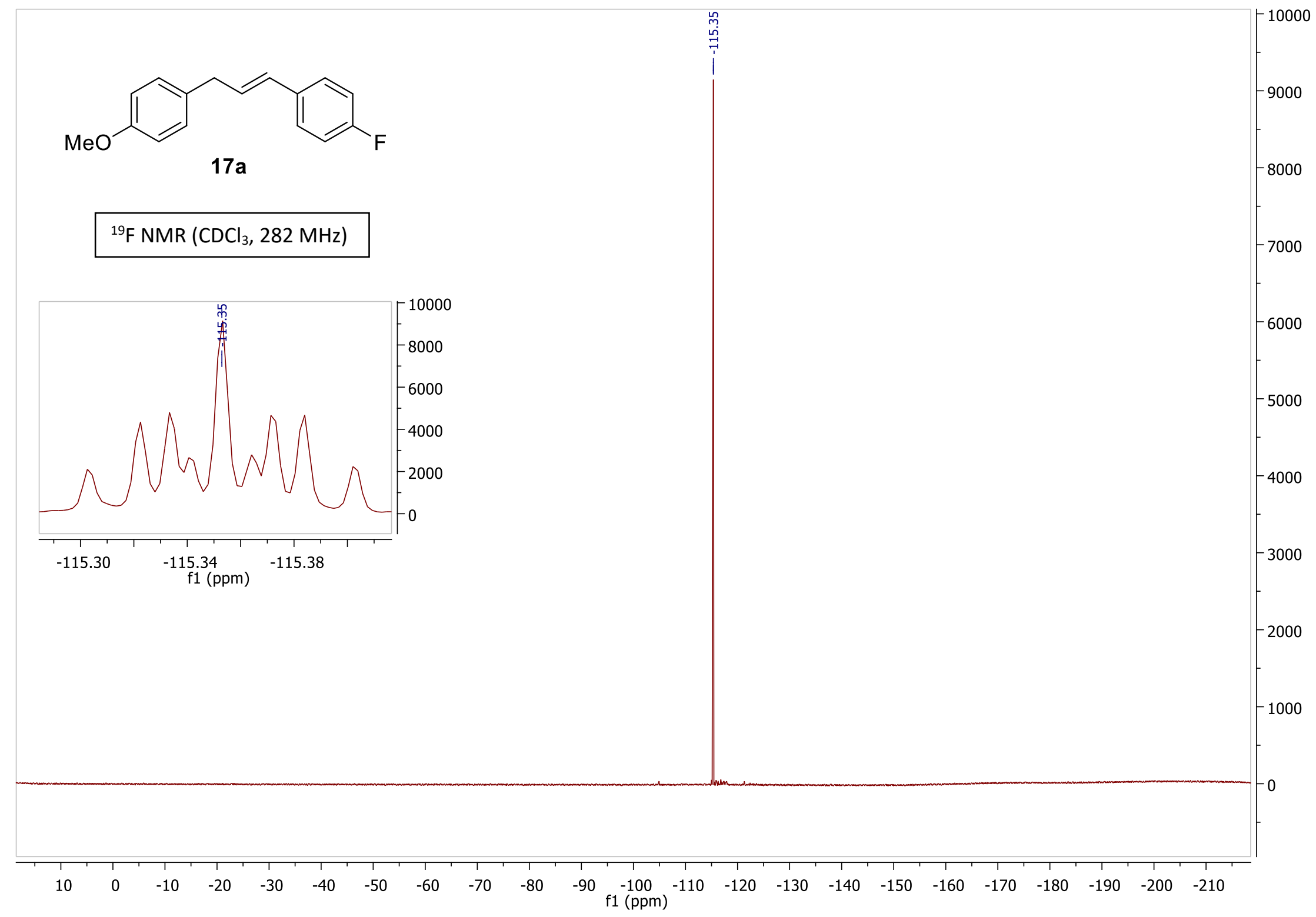




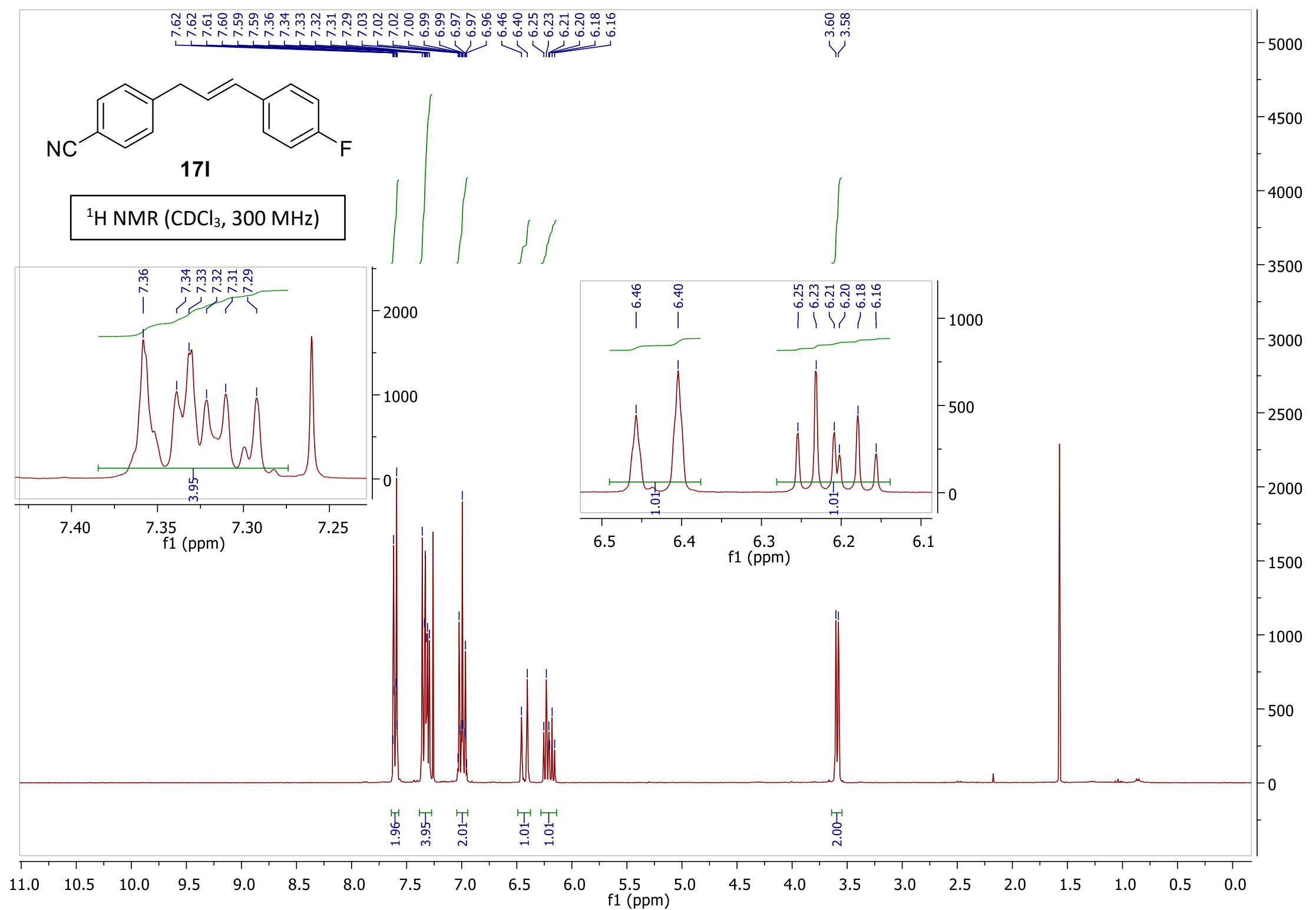




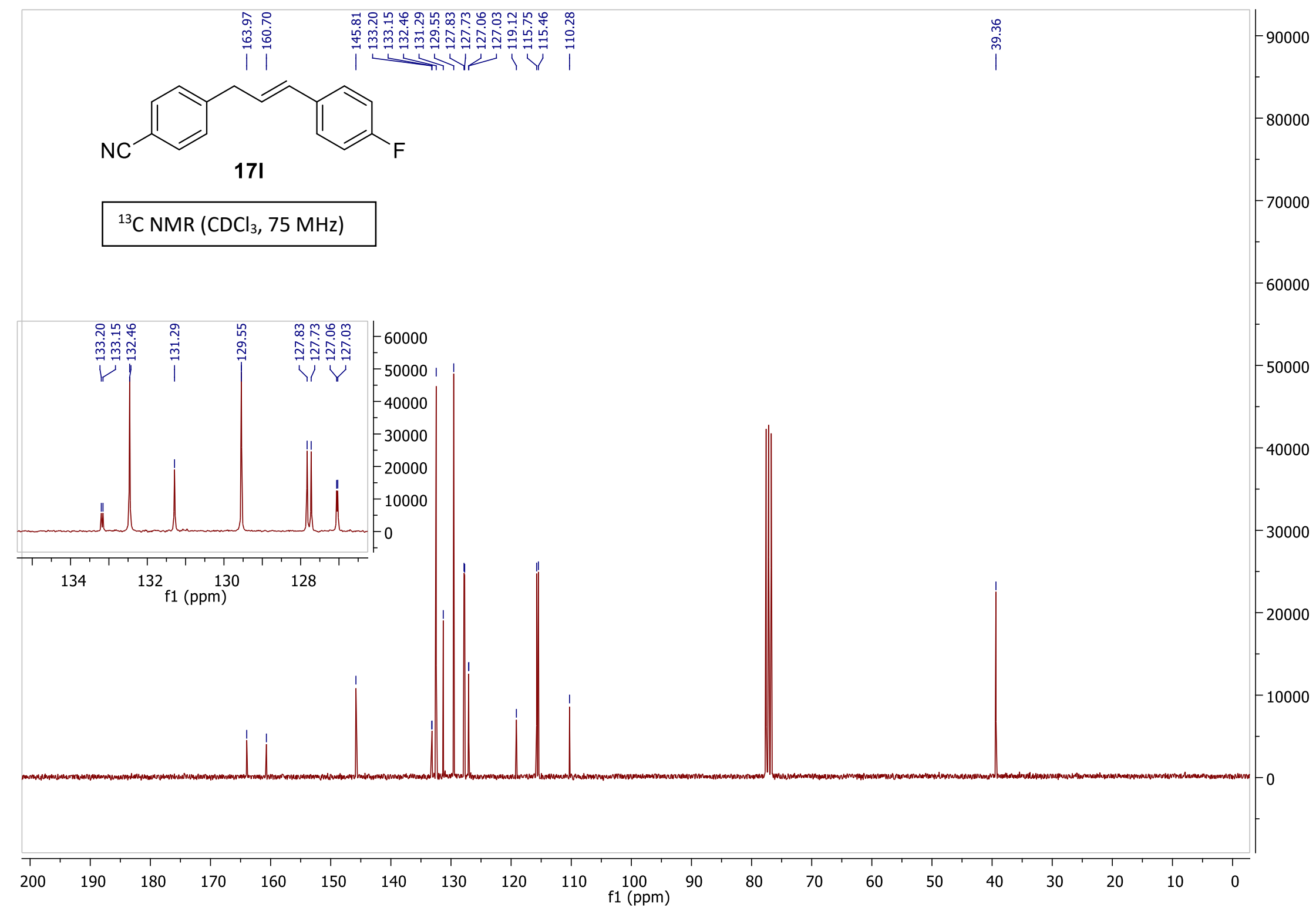




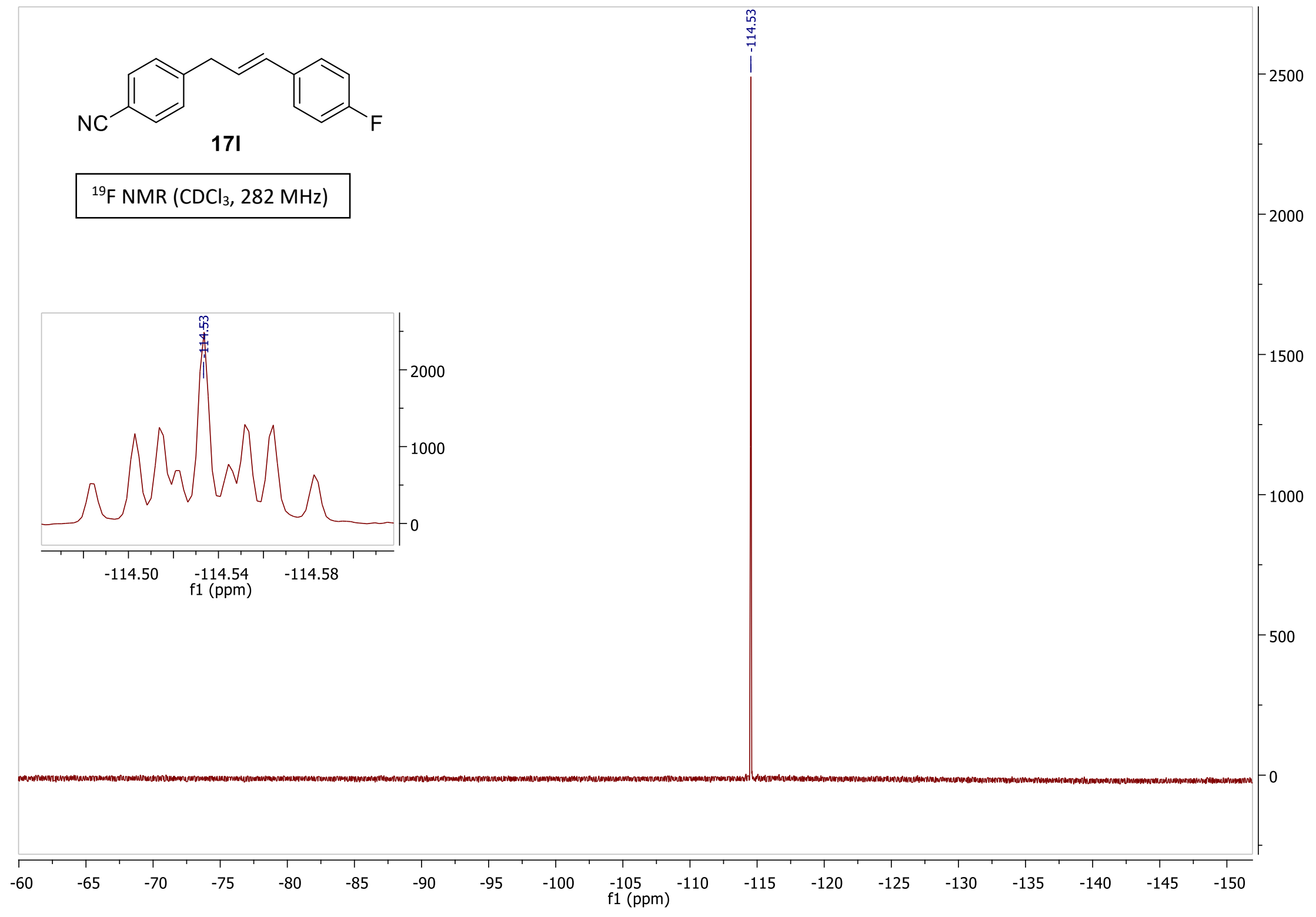


Data:2002028

Comment:SP-188 GC/EI

Description:

lonization Mode:EI+

History:Average(MS[1] 11.90..11.94)-1*Average(MS[1] 11.78..11.81),\#0-

Charge number: 1

Tolerance:5.00[mDa]

Element: ${ }^{12} \mathrm{C}: 0$.. 50, ${ }^{1} \mathrm{H}: 0$.. 100, ${ }^{16} \mathrm{O}: 0$.. 3

$3 a$

\begin{tabular}{|c|c|c|r|r|r|r|}
\hline Mass & Intensity & Calc. Mass & $\begin{array}{c}\text { Mass Difference } \\
{[\mathrm{mDa}]}\end{array}$ & $\begin{array}{c}\text { Mass Difference } \\
{[\mathrm{ppm}]}\end{array}$ & Possible Formula & Unsaturation Number \\
\hline 188.12066 & 1358201.55 & 188.12011 & 0.55 & 2.90 & ${ }^{12} \mathrm{C}_{13}{ }^{1} \mathrm{H}_{16}{ }^{16} \mathrm{O}_{1}$ & 6.0 \\
\hline
\end{tabular}

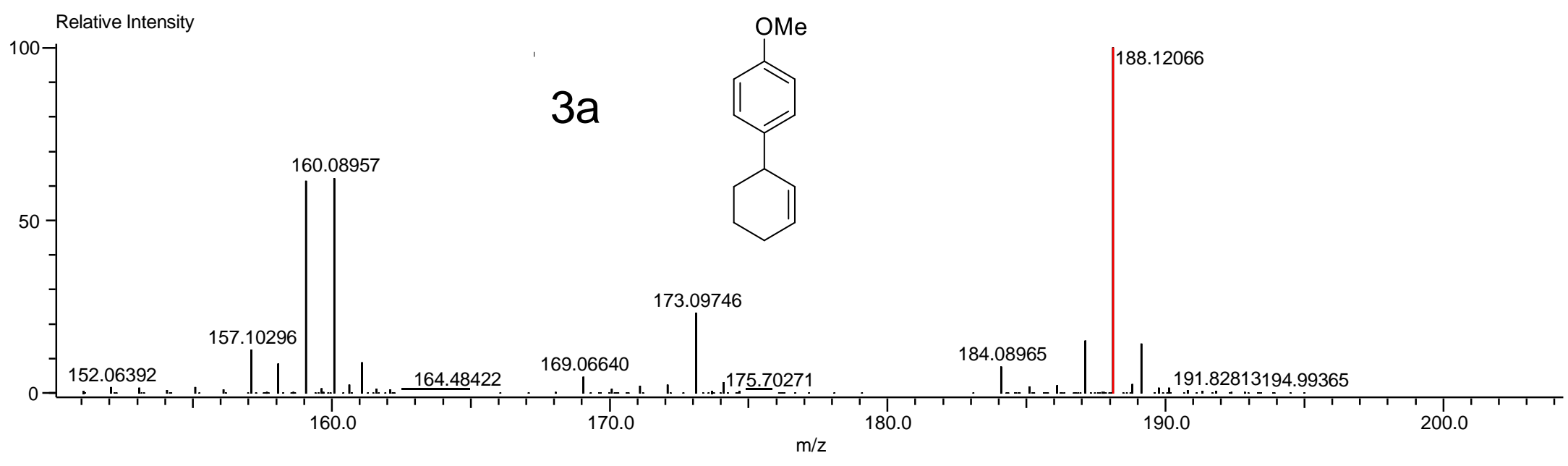

Acquired:2/14/2020 3:04:49 PM

Operator:AccuTOF

$\mathrm{m} / \mathrm{z}$ Calibration File:CalibEI220120

Created:2/14/2020 3:34:22 PM

Created by:AccuTOF

Unsaturation Number:-2.0 .. 15.0 (Fraction:Both) 
Data: 1910049

Comment:SP-192 GC/Cl tBuH

Description:

Ionization Mode: $\mathrm{Cl}+$

History:Average(MS[1] 10.27..10.30)-1*Average(MS[1] 10.07..10.12),\#0-

Charge number:1

Element: ${ }^{12} \mathrm{C}: 0$.. 50, ${ }^{1} \mathrm{H}: 0$.. 100, ${ }^{16} \mathrm{O}: 0$.. 5

Tolerance: $5.00[\mathrm{mDa}]$
$100-$

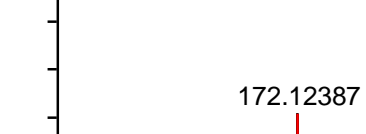

50

2.12387

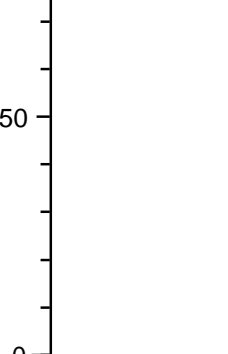

$3 b$

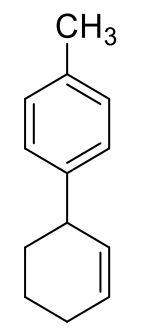

Acquired:12:00:00 AM

Operator:AccuTOF

$\mathrm{m} / \mathrm{z}$ Calibration File:Calib191004

Created:10/17/2019 2:21:21 PM

Created by:AccuTOF

Unsaturation Number:0.0 .. 10.0 (Fraction:Both)

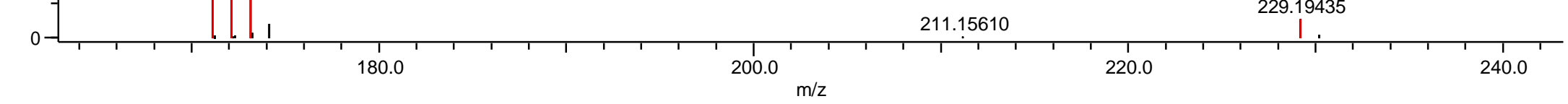

\begin{tabular}{|r|r|r|r|r|r|r|}
\hline Mass & Intensity & Calc. Mass & $\begin{array}{c}\text { Mass Difference } \\
{[\mathrm{mDa}]}\end{array}$ & $\begin{array}{c}\text { Mass Difference } \\
{[\mathrm{ppm}]}\end{array}$ & Possible Formula & Unsaturation Number \\
\hline 171.11668 & 25269.09 & 171.11738 & -0.69 & $-4.04^{12} \mathrm{C}_{13}{ }^{1} \mathrm{H}_{15}$ & 6.5 \\
\hline 172.12387 & 27487.98 & 172.12520 & -1.33 & $-7.75^{12} \mathrm{C}_{13}{ }^{1} \mathrm{H}_{16}$ & 6.0 \\
\hline 173.13098 & 12186.60 & 173.13303 & -2.05 & $-11.83^{12} \mathrm{C}_{13}{ }^{1} \mathrm{H}_{17}$ & 5.5 \\
\hline 229.19435 & 2046.01 & 229.19563 & -1.28 & $-5.58^{12} \mathrm{C}_{17} 1^{1} \mathrm{H}_{25}$ & 5.5 \\
\hline
\end{tabular}


Data: 1912035

Comment:SP-198 GC/EI

Description:

lonization Mode:EI+

History:Average(MS[1] 16.58..16.62)

Charge number: 1

Element: ${ }^{12} \mathrm{C}: 0$.. 50, ${ }^{1} \mathrm{H}: 0$.. 100, ${ }^{14} \mathrm{~N}: 0$.. 3

Tolerance:5.00[mDa]

Acquired:12/19/2019 4:18:18 PM

Operator:AccuTOF

$\mathrm{m} / \mathrm{z}$ Calibration File:Calib191200

Created:12/19/2019 4:44:42 PM

Created by:AccuTOF

Unsaturation Number:-2.0 .. 15.0 (Fraction:Both)
$100-$

00

50

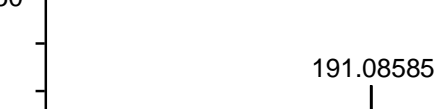

206.10847

$3 c$<smiles>C1=CC(c2ccc(-c3ccccc3)cc2)CCC1</smiles>

226.07729230 .10865

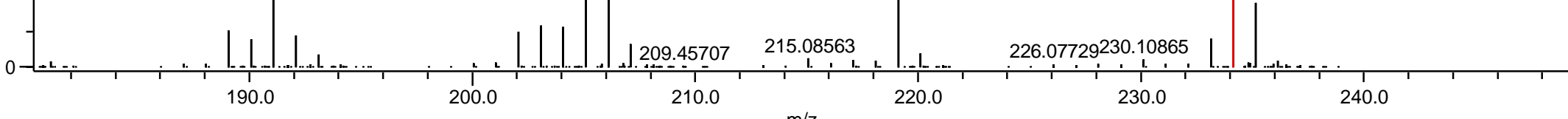

\begin{tabular}{|c|c|r|r|r|r|r|}
\hline Mass & Intensity & Calc. Mass & $\begin{array}{c}\text { Mass Difference } \\
{[\mathrm{mDa}]}\end{array}$ & $\begin{array}{c}\text { Mass Difference } \\
{[\mathrm{ppm}]}\end{array}$ & Possible Formula & Unsaturation Number \\
\hline 234.13987 & 657570.18 & 234.14085 & -0.98 & $-4.17^{12} \mathrm{C}_{18}{ }^{1} \mathrm{H}_{18}$ & 10.0 \\
\hline
\end{tabular}


Data:1912051

Comment:SP-206 GC/E

Description:

Ionization Mode:El+

History:Average(MS[1] 9.39..9.42)

Charge number: 1

Element: ${ }^{12} \mathrm{C}: 0$.. 50, ${ }^{1} \mathrm{H}: 0$.. 100, ${ }^{19} \mathrm{~F}: 0$.. 3

Relative Intensity

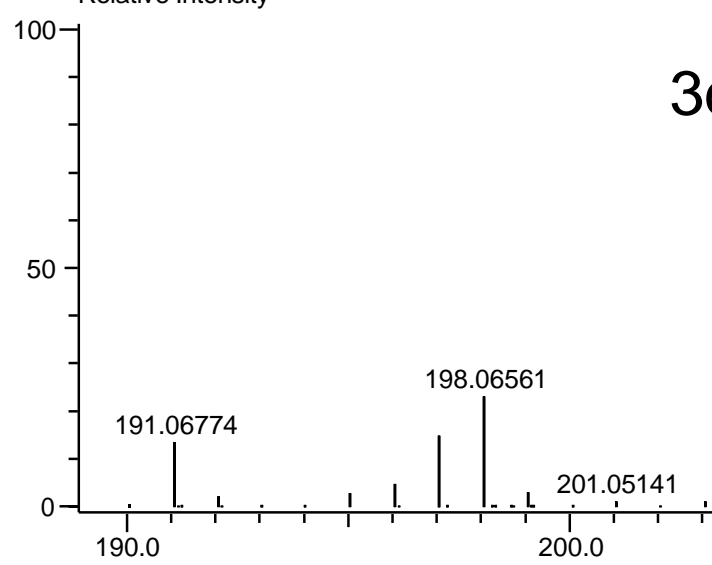

$3 d$

Tolerance:5.00[mDa]

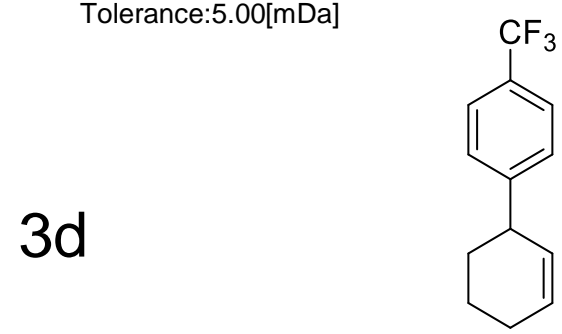

211.07327

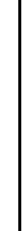

\begin{tabular}{l|l}
205.08308 & 209.05789
\end{tabular}

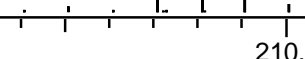

Acquired:12:00:00 AM

Operator:AccuTOF

$\mathrm{m} / \mathrm{z}$ Calibration File:Calib1912002

Created:12/20/2019 10:57:18 AM

Created by:AccuTOF

Unsaturation Number:-2.0 .. 15.0 (Fraction:Both)

219.03454222 .06526

220.0

230.0

\begin{tabular}{|c|r|r|r|r|r|r|}
\hline Mass & Intensity & Calc. Mass & $\begin{array}{c}\text { Mass Difference } \\
{[\mathrm{mDa}]}\end{array}$ & $\begin{array}{c}\text { Mass Difference } \\
{[\mathrm{ppm}]}\end{array}$ & Possible Formula & Unsaturation Number \\
\hline 226.09633 & 213323.08 & 226.09693 & -0.60 & -2.67 & ${ }^{12} \mathrm{C}_{13}{ }^{1} \mathrm{H}_{13}{ }^{19} \mathrm{~F}_{3}$ & 6.0 \\
\hline
\end{tabular}


Data:1912038

Comment:SP-200 GC/EI

Description:

lonization Mode:El+

History:Average(MS[1] 14.87..14.90)

Charge number: 1

Element: ${ }^{12} \mathrm{C}: 0$.. 50, ${ }^{1} \mathrm{H}: 0$.. 100, ${ }^{79} \mathrm{Br}: 0$.. 1, ${ }^{81} \mathrm{Br}: 0$.. 1

Tolerance: $5.00[\mathrm{mDa}]$

\section{Relative Intensity}
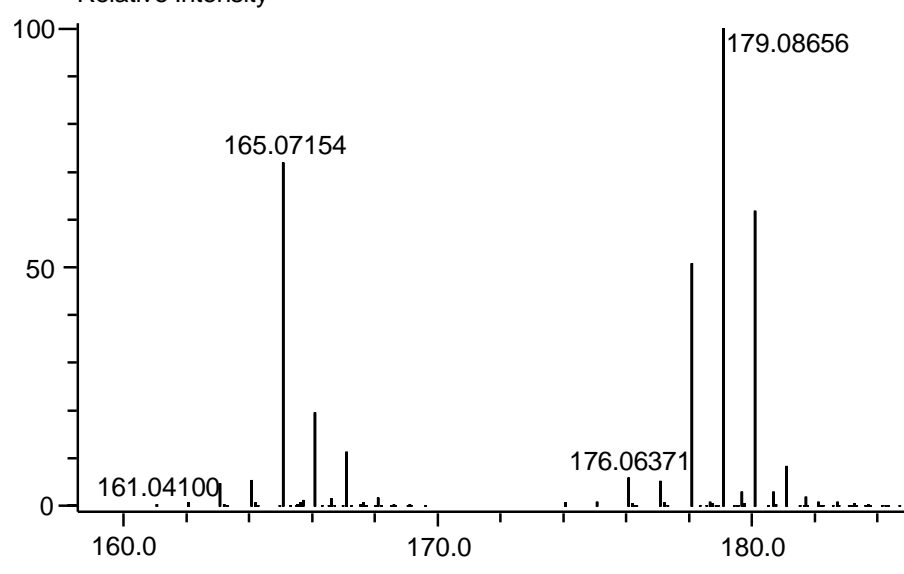

3e

\begin{tabular}{|c|c|r|r|r|r|r|}
\hline Mass & Intensity & Calc. Mass & $\begin{array}{c}\text { Mass Difference } \\
{[\mathrm{mDa}]}\end{array}$ & $\begin{array}{c}\text { Mass Difference } \\
{[\mathrm{ppm}]}\end{array}$ & Possible Formula & Unsaturation Number \\
\hline 208.12502 & 541624.22 & 208.12520 & -0.18 & -0.84 & ${ }^{12} \mathrm{C}_{16}{ }^{1} \mathrm{H}_{16}$ & 9.0 \\
\hline
\end{tabular}

Acquired:12:00:00 AM

Operator:AccuTOF

m/z Calibration File:Calib1912002

Created:12/20/2019 9:56:48 AM

Created by:AccuTOF

Unsaturation Number:-2.0 .. 15.0 (Fraction:Both) 
Data: 1910046

Comment:SP-180 GC/Cl tBuH

Description:

Ionization Mode: $\mathrm{Cl}+$

History:Average(MS[1] 14.29..14.32)-1*Average(MS[1] 14.01..14.05),\#0-

Charge number: 1

Element: ${ }^{12} \mathrm{C}: 0$.. 50, ${ }^{1} \mathrm{H}: 0$.. 100, ${ }^{16} \mathrm{O}: 0$.. 5

Tolerance:5.00[mDa]

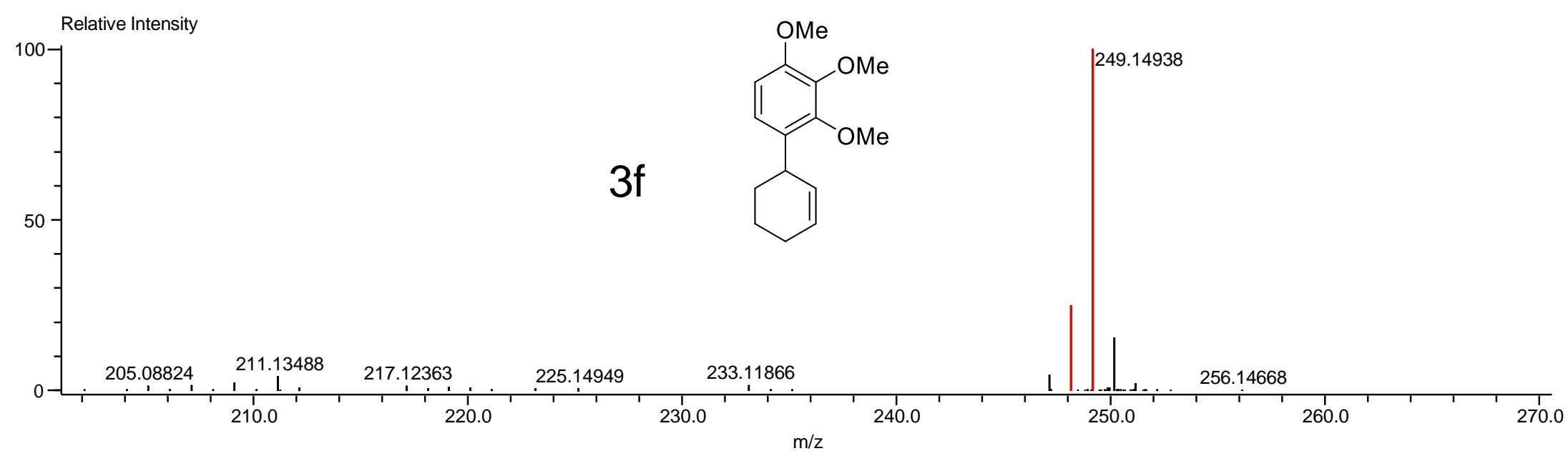

Acquired:12:00:00 AM

Operator:AccuTOF

m/z Calibration File:Calib191004

Created:10/17/2019 10:45:14 AM

Created by:AccuTOF

Unsaturation Number:0.0 .. 10.0 (Fraction:Both)

\begin{tabular}{|l|r|r|r|r|r|r|}
\hline Mass & Intensity & Calc. Mass & $\begin{array}{c}\text { Mass Difference } \\
{[\mathrm{mDa}]}\end{array}$ & $\begin{array}{c}\text { Mass Difference } \\
{[\mathrm{ppm}]}\end{array}$ & Possible Formula & Unsaturation Number \\
\hline 248.14157 & 59504.05 & 248.14124 & 0.33 & $1.32^{12} \mathrm{C}_{15}{ }^{1} \mathrm{H}_{20}{ }^{16} \mathrm{O}_{3}$ & 6.0 \\
\hline 249.14938 & 239730.95 & 249.14907 & 0.31 & 1.24 & ${ }^{12} \mathrm{C}_{15}{ }^{1} \mathrm{H}_{21}{ }^{16} \mathrm{O}_{3}$ & 5.5 \\
\hline
\end{tabular}


Data:1910048

Comment:SP-184 GC/Cl tBuH

Description:

Ionization Mode: $\mathrm{Cl}_{+}$

History:Average(MS[1] 11.77..11.80)-1*Average(MS[1] 11.66..11.67),\#0-

Charge number: 1

Element: ${ }^{12} \mathrm{C}: 0$.. 50, ${ }^{1} \mathrm{H}: 0 . .100,{ }^{16} \mathrm{O}: 0$.. 5

Tolerance: $5.00[\mathrm{mDa}]$
Acquired:12:00:00 AM

Operator:AccuTOF

$\mathrm{m} / \mathrm{z}$ Calibration File:Calib191004

Created:10/17/2019 11:33:31 AM

Created by:AccuTOF

Unsaturation Number:0.0 .. 10.0 (Fraction:Both)

Relative Intensity

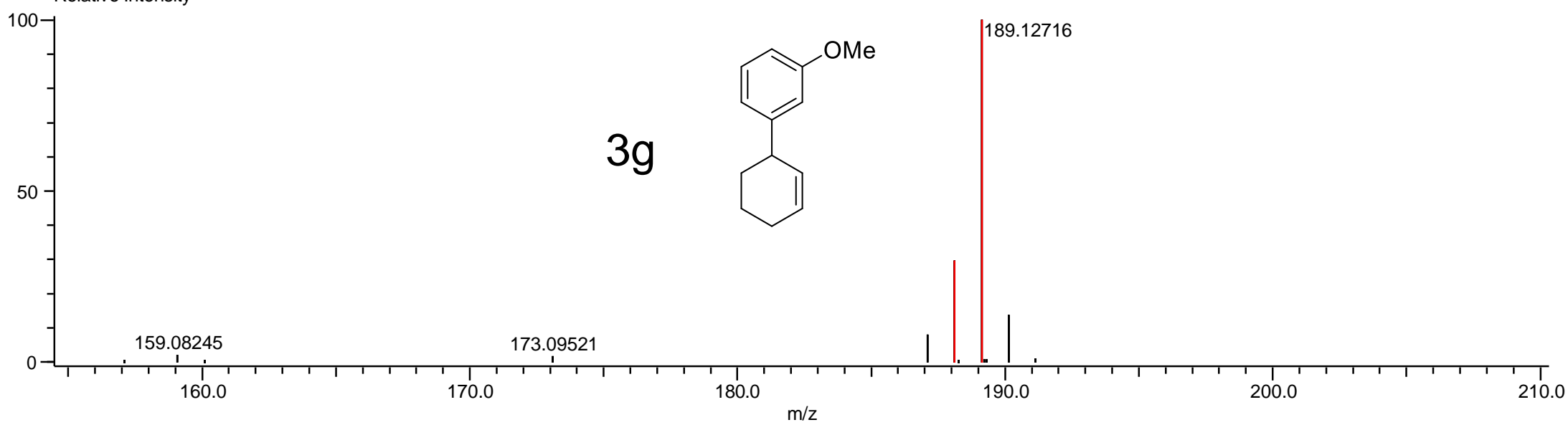

\begin{tabular}{|c|r|r|r|r|r|r|}
\hline Mass & Intensity & Calc. Mass & $\begin{array}{c}\text { Mass Difference } \\
{[\mathrm{mDa}]}\end{array}$ & $\begin{array}{c}\text { Mass Difference } \\
{[\mathrm{ppm}]}\end{array}$ & Possible Formula & Unsaturation Number \\
\hline 188.11936 & 7612.06 & 188.12011 & -0.76 & $-4.03^{12} \mathrm{C}_{13}{ }^{1} \mathrm{H}_{16}{ }^{16} \mathrm{O}_{1}$ & 6.0 \\
\hline 189.12716 & 25740.45 & 189.12794 & -0.78 & -4.11 & ${ }^{12} \mathrm{C}_{13}{ }^{1} \mathrm{H}_{17}{ }^{16} \mathrm{O}_{1}$ & 5.5 \\
\hline
\end{tabular}


Data:1910051

Comment:SP-182 GC/Cl tBuH

Description:

Ionization Mode:Cl+

History:Average(MS[1] 8.90..8.93)

Charge number: 1

Element: ${ }^{12} \mathrm{C}: 0$.. 50, ${ }^{1} \mathrm{H}: 0$.. 100, ${ }^{19} \mathrm{~F}: 0$.. 2

Tolerance:5.00[mDa]

\section{Relative Intensity}

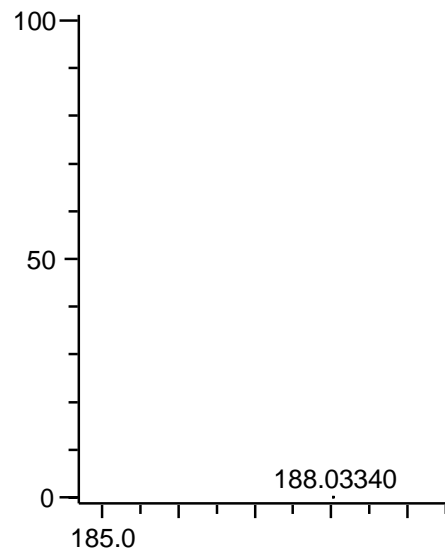

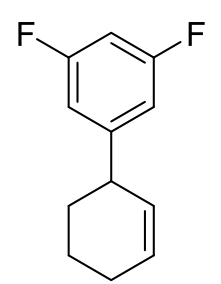

$3 h$
Acquired:10/17/2019 2:51:07 PM

Operator:AccuTOF

m/z Calibration File:Calib191004

Created:10/17/2019 3:22:15 PM

Created by:AccuTOF

Unsaturation Number:0.0 .. 10.0 (Fraction:Both)

\begin{tabular}{|c|r|r|r|r|r|r|}
\hline Mass & Intensity & Calc. Mass & $\begin{array}{c}\text { Mass Difference } \\
{[\mathrm{mDa}]}\end{array}$ & $\begin{array}{c}\text { Mass Difference } \\
{[\mathrm{ppm}]}\end{array}$ & Possible Formula & Unsaturation Number \\
\hline 193.08414 & 19645.36 & 193.08288 & 1.26 & $6.511^{12} \mathrm{C}_{12}{ }^{1} \mathrm{H}_{11}{ }^{19} \mathrm{~F}_{2}$ & 6.5 \\
\hline 194.09176 & 77409.80 & 194.09071 & 1.05 & 5.41 & ${ }^{12} \mathrm{C}_{12}{ }^{1} \mathrm{H}_{12}{ }^{19} \mathrm{~F}_{2}$ & 6.0 \\
\hline 195.09932 & 91470.84 & 195.09853 & 0.79 & 4.04 & ${ }^{12} \mathrm{C}_{12}{ }^{1} \mathrm{H}_{13}{ }^{19} \mathrm{~F}_{2}$ & 5.5 \\
\hline
\end{tabular}


Data:2002018

Comment:SP-243 GC/E

Description:

lonization Mode:EI+

History:Average(MS[1] 11.82..11.86)-1*Average(MS[1] 11.70..11.74),\#0-

Charge number: 1

Element: ${ }^{12} \mathrm{C}: 0$.. 50, ${ }^{1} \mathrm{H}: 0$.. 100, ${ }^{19} \mathrm{~F}: 0$.. 1, ${ }^{14} \mathrm{~N}: 0$.. 1, ${ }^{16} \mathrm{O}: 0$..

Tolerance:5.00[mDa]

\section{Relative Intensity}

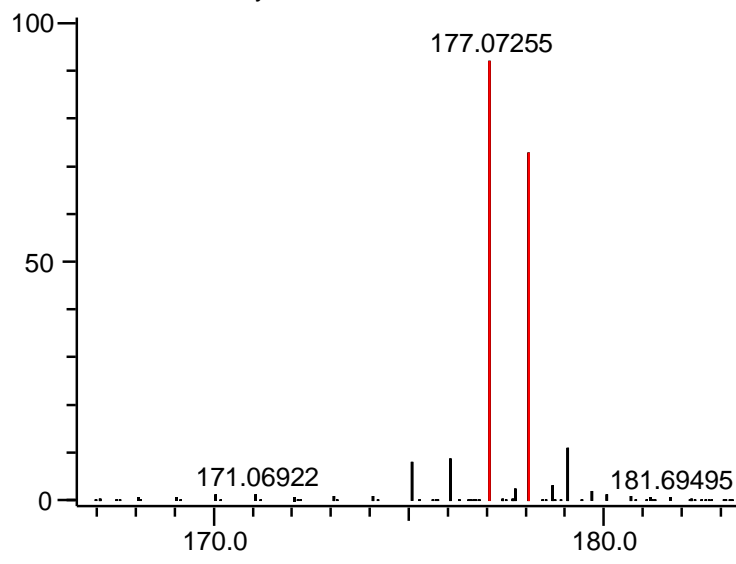

$3 i$

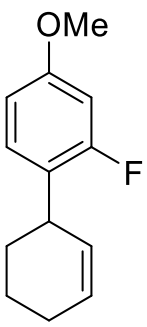

Acquired:12:00:00 AM

Operator:AccuTOF

$\mathrm{m} / \mathrm{z}$ Calibration File:CalibEI220120

Created:2/7/2020 10:48:09 AM

Created by:AccuTOF

Unsaturation Number:-2.0 .. 9.0 (Fraction:Both)

\begin{tabular}{|c|c|c|c|c|c|c|}
\hline Mass & Intensity & Calc. Mass & $\begin{array}{c}\text { Mass Difference } \\
{[\mathrm{mDa}]}\end{array}$ & $\begin{array}{c}\text { Mass Difference } \\
{[\mathrm{ppm}]}\end{array}$ & Possible Formula & Unsaturation Number \\
\hline 177.07255 & 1350756.56 & 177.07157 & 0.98 & 5.56 & ${ }^{12} \mathrm{C}_{11}{ }^{1} \mathrm{H}_{10^{19}} \mathrm{~F}_{1}{ }^{16} \mathrm{O}_{1}$ & 6.5 \\
\hline 178.07977 & 1069854.52 & 178.07939 & 0.38 & 2.14 & ${ }^{12} \mathrm{C}_{11}{ }^{1} \mathrm{H}_{11}{ }^{19} \mathrm{~F}_{1}{ }^{16} \mathrm{O}_{1}$ & 6.0 \\
\hline 191.08775 & 481838.67 & 191.08722 & 0.53 & 2.79 & ${ }^{12} \mathrm{C}_{12}{ }^{1} \mathrm{H}_{12}{ }^{19} \mathrm{~F}_{1}{ }^{16} \mathrm{O}_{1}$ & 6.5 \\
\hline 206.11085 & 1470016.97 & 206.11069 & 0.16 & 0.78 & ${ }^{12} \mathrm{C}_{13}{ }^{1} \mathrm{H}_{15^{19}} \mathrm{~F}_{1}{ }^{16} \mathrm{O}_{1}$ & 6.0 \\
\hline
\end{tabular}


Data: 1910050

Comment:SP-193 GC/Cl tBuH

Description:

Ionization Mode: $\mathrm{Cl}_{+}$

History:Average(MS[1] 13.94..13.98)

Charge number:1

Element: ${ }^{12} \mathrm{C}: 0$.. 50, ${ }^{1} \mathrm{H}: 0$.. 100, ${ }^{16} \mathrm{O}: 0$. . 5

Tolerance:5.00[mDa]
Acquired:12:00:00 AM

Operator:AccuTOF

m/z Calibration File:Calib191004

Created:10/17/2019 2:44:26 PM

Created by:AccuTOF

Unsaturation Number:0.0 .. 10.0 (Fraction:Both)

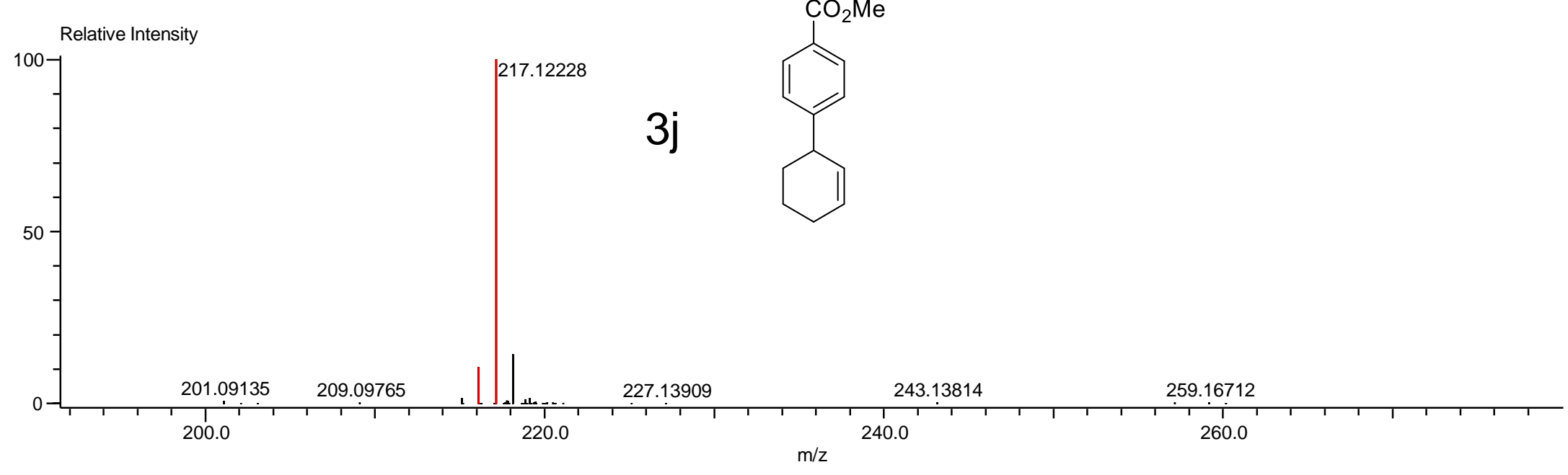

\begin{tabular}{|c|c|c|c|c|c|c|}
\hline Mass & Intensity & Calc. Mass & $\begin{array}{l}\text { Mass Difference } \\
{[\mathrm{mDa}]}\end{array}$ & $\begin{array}{l}\text { Mass Difference } \\
{[\mathrm{ppm}]}\end{array}$ & Possible Formula & Unsaturation Number \\
\hline 216.11454 & 46408.79 & 216.11503 & -0.49 & -2.2 & ${ }^{12} \mathrm{C}_{14}{ }^{1} \mathrm{H}_{16}{ }^{16} \mathrm{O}_{2}$ & 7.0 \\
\hline 217.12228 & 444263.22 & 217.12285 & -0.58 & -2.66 & ${ }^{12} \mathrm{C}_{14}{ }^{1} \mathrm{H}_{17^{16}} \mathrm{O}_{2}$ & 6.5 \\
\hline
\end{tabular}


Data:1912056

Comment:SP-219 GC/E

Description:

Ionization Mode:El+

History:Average(MS[1] 13.72..13.76)-1*Average(MS[1] 13.60..13.64),\#0-

Charge number: 1

Tolerance:2.00[mDa]

Element: ${ }^{12} \mathrm{C}: 0$.. 50, ${ }^{1} \mathrm{H}: 0$.. 100, ${ }^{16} \mathrm{O}: 0$.. 6

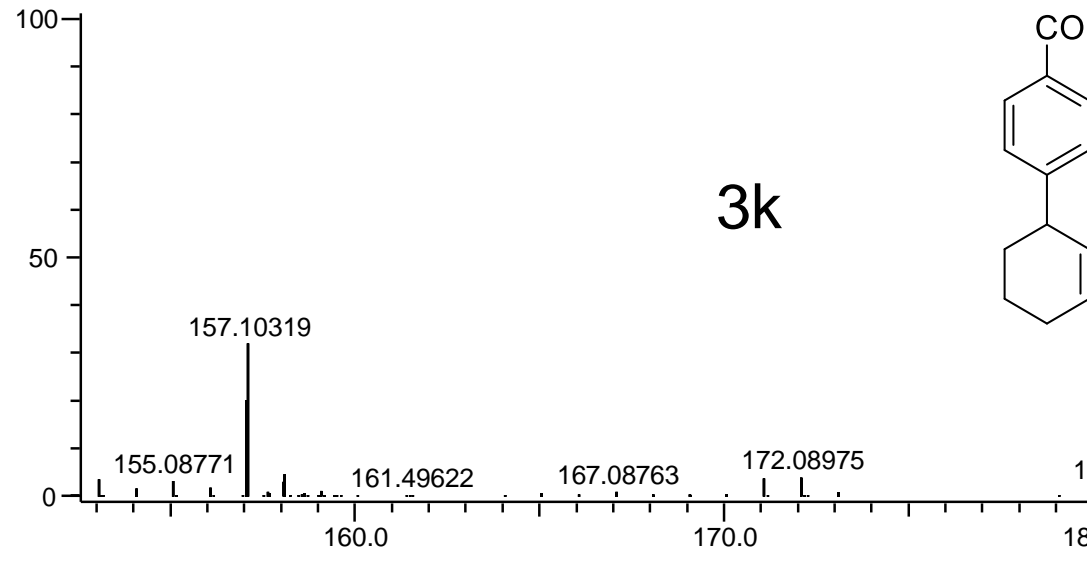

COMe 185.09758

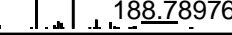

200.12060

190.0

\begin{tabular}{|c|c|r|r|r|r|r|}
\hline Mass & Intensity & Calc. Mass & $\begin{array}{c}\text { Mass Difference } \\
{[\mathrm{mDa}]}\end{array}$ & $\begin{array}{c}\text { Mass Difference } \\
{[\mathrm{ppm}]}\end{array}$ & Possible Formula & Unsaturation Number \\
\hline 200.12060 & 303338.77 & 200.12011 & 0.49 & $2.43^{12} \mathrm{C}_{14}{ }^{1} \mathrm{H}_{16}{ }^{16} \mathrm{O}_{1}$ & 7.0 \\
\hline
\end{tabular}


Data:1912034

Comment:SP-197 GC/EI

Description:

Ionization Mode:El+

History:Average(MS[1] 12.87..12.90)

Charge number: 1

Element: ${ }^{12} \mathrm{C}: 0$.. 50, ${ }^{1} \mathrm{H}: 0$.. 100, ${ }^{14} \mathrm{~N}: 0$.. 3

Relative Intensity

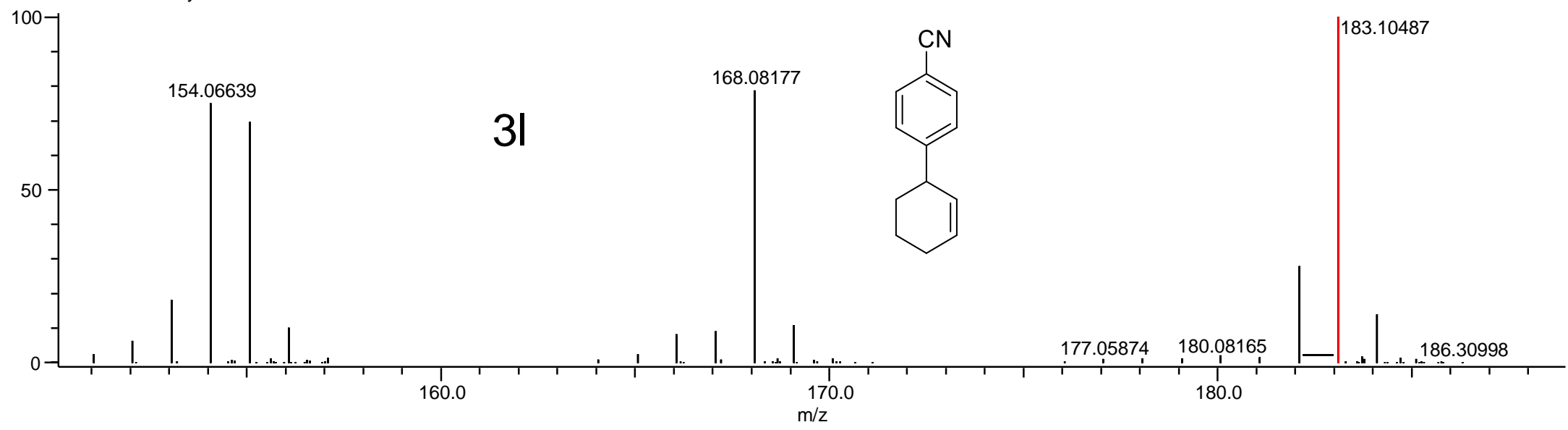

Acquired:12:00:00 AM

Operator:AccuTOF

m/z Calibration File:Calib1912001

Created:12/19/2019 4:06:40 PM

Created by:AccuTOF

Unsaturation Number:-2.0 .. 15.0 (Fraction:Both)
Tolerance:5.00[mDa]

\begin{tabular}{|c|c|r|r|r|r|r|}
\hline Mass & Intensity & Calc. Mass & $\begin{array}{c}\text { Mass Difference } \\
{[\mathrm{mDa}]}\end{array}$ & $\begin{array}{c}\text { Mass Difference } \\
{[\mathrm{ppm}]}\end{array}$ & Possible Formula & Unsaturation Number \\
\hline 183.10487 & 207738.32 & 183.10480 & 0.07 & $0.39^{12} \mathrm{C}_{13^{1}} \mathrm{H}_{13}{ }^{14} \mathrm{~N}_{1}$ & 8.0 \\
\hline
\end{tabular}


Data:1912036

Comment:SP-199 GC/EI

Description:

Ionization Mode:El+

History:Average(MS[1] 12.36..12.39)

Charge number: 1

Element: ${ }^{12} \mathrm{C}: 0$.. 50, ${ }^{1} \mathrm{H}: 0$.. 100, ${ }^{79} \mathrm{Br}: 0$.. 1, ${ }^{81} \mathrm{Br}: 0$.. 1

Tolerance:5.00[mDa]
Acquired:12:00:00 AM

Operator:AccuTOF

m/z Calibration File:Calib1912001

Created:12/19/2019 5:13:18 PM

Created by:AccuTOF

Unsaturation Number:-2.0 .. 15.0 (Fraction:Both)

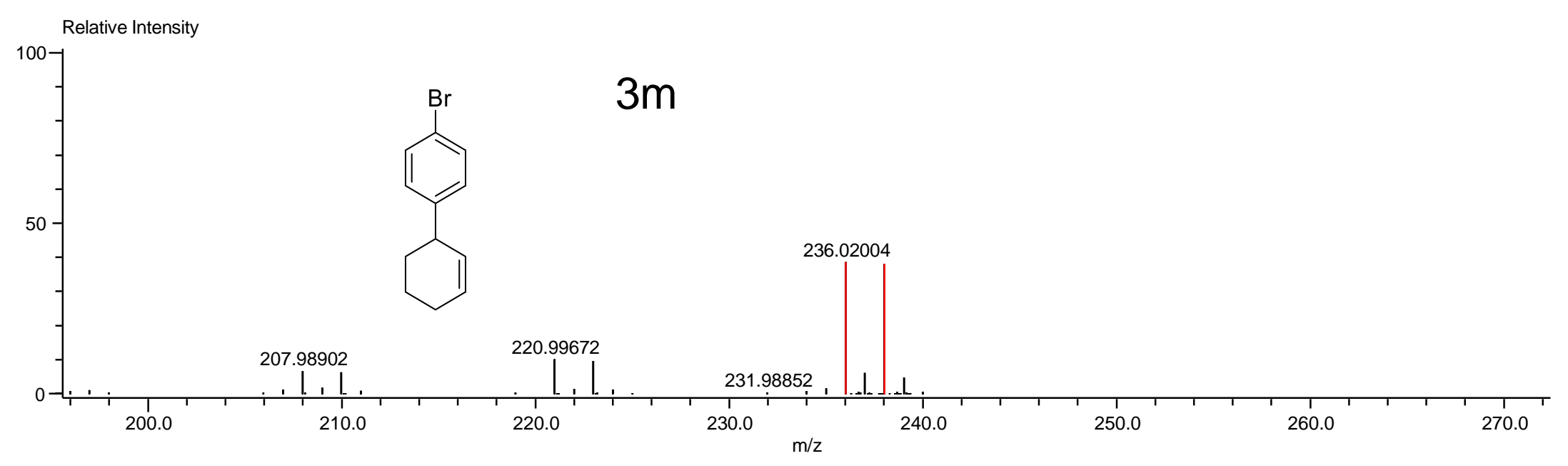

\begin{tabular}{|c|c|r|r|r|r|r|}
\hline Mass & Intensity & Calc. Mass & $\begin{array}{c}\text { Mass Difference } \\
{[\mathrm{mDa}]}\end{array}$ & $\begin{array}{c}\text { Mass Difference } \\
{[\mathrm{ppm}]}\end{array}$ & Possible Formula & Unsaturation Number \\
\hline 236.02004 & 129129.61 & 236.02006 & -0.03 & $-0.111^{12} \mathrm{C}_{12}{ }^{1} \mathrm{H}_{13}{ }^{79} \mathrm{Br}_{1}$ & 6.0 \\
\hline 238.01806 & 127580.68 & 238.01802 & 0.04 & $0.17^{12} \mathrm{C}_{12}{ }^{1} \mathrm{H}_{13}{ }^{81} \mathrm{Br}_{1}$ & 6.0 \\
\hline
\end{tabular}


Data:1912033

Comment:SP-195 GC/EI

Description:

Ionization Mode:EI+

History:Average(MS[1] 9.20..9.22)-1*Average(MS[1] 9.11..9.13),\#0-

Charge number: 1

Element: ${ }^{12} \mathrm{C}: 0$.. 50, ${ }^{1} \mathrm{H}: 0$.. 100, ${ }^{19} \mathrm{~F}: 0$.. 1

Tolerance:5.00[mDa]

Relative Intensity

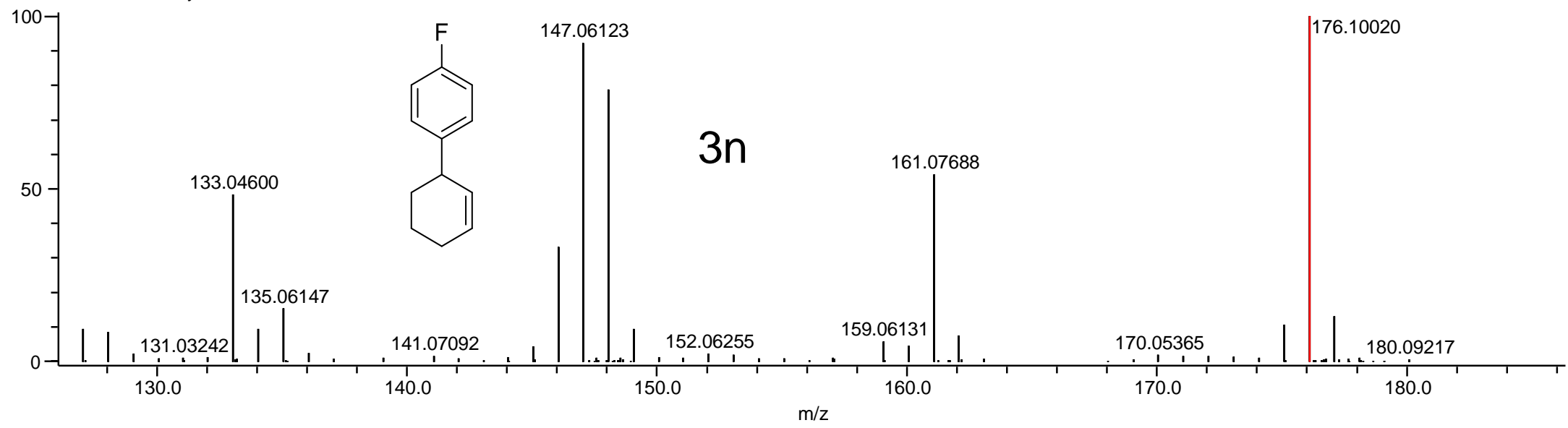

\begin{tabular}{|c|c|c|r|r|r|r|}
\hline Mass & Intensity & Calc. Mass & $\begin{array}{c}\text { Mass Difference } \\
{[\mathrm{mDa}]}\end{array}$ & $\begin{array}{c}\text { Mass Difference } \\
{[\mathrm{ppm}]}\end{array}$ & Possible Formula & Unsaturation Number \\
\hline 176.10020 & 113811.47 & 176.10013 & 0.08 & $0.44{ }^{12} \mathrm{C}_{12}{ }^{1} \mathrm{H}_{13}{ }^{19} \mathrm{~F}_{1}$ & 6.0 \\
\hline
\end{tabular}

Acquired:12:00:00 AM

Operator:AccuTOF

m/z Calibration File:Calib1912001

Created:12/19/2019 3:39:06 PM

Created by:AccuTOF

Unsaturation Number:-2.0 .. 15.0 (Fraction:Both)
76.10020

176.10013

0.08 
Data: 1912057

Comment:SP-220 GC/EI

Description:

Ionization Mode:El+

History:Average(MS[1] 11.38..11.41)-1*Average(MS[1] 11.26..11.29),\#0-

Charge number: 1

Element: ${ }^{12} \mathrm{C}: 0$.. 50, ${ }^{1} \mathrm{H}: 0$.. 100, ${ }^{35} \mathrm{Cl}: 0$.. 1, ${ }^{37} \mathrm{Cl}: 0$.. 1

Tolerance:2.00[mDa]

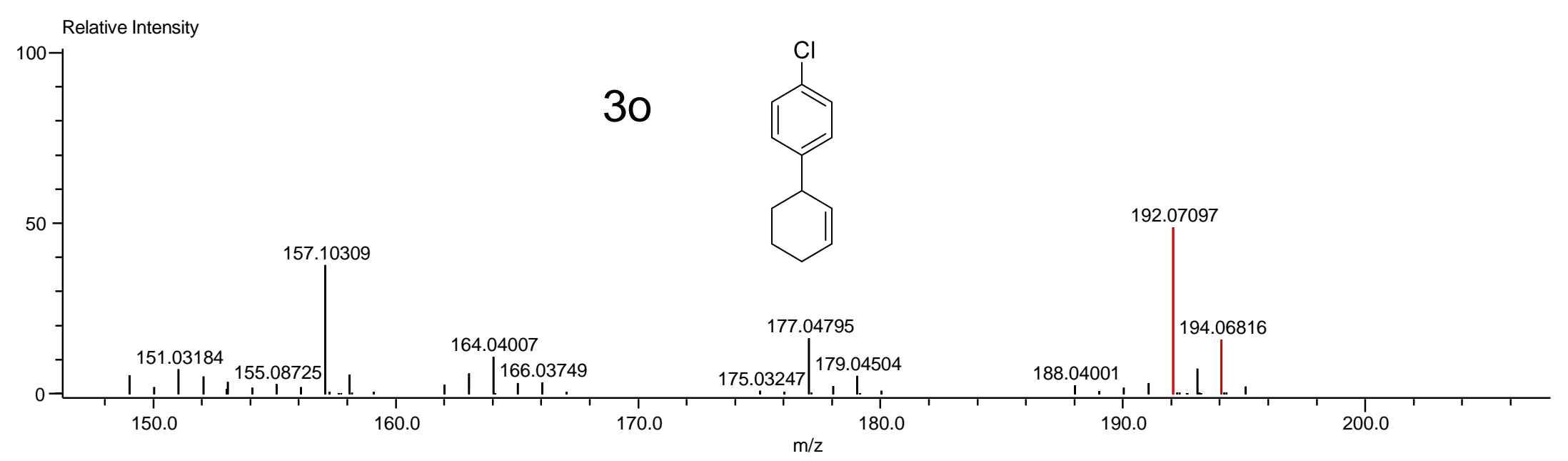

Acquired:12/20/2019 1:27:06 PM

Operator:AccuTOF

m/z Calibration File:Calib1912002

Created:12/20/2019 2:13:44 PM

Created by:AccuTOF

Unsaturation Number:-2.0 .. 15.0 (Fraction:Both)

\begin{tabular}{|c|r|r|r|r|r|r|}
\hline Mass & Intensity & Calc. Mass & $\begin{array}{c}\text { Mass Difference } \\
{[\mathrm{mDa}]}\end{array}$ & $\begin{array}{c}\text { Mass Difference } \\
{[\mathrm{ppm}]}\end{array}$ & Possible Formula & Unsaturation Number \\
\hline 192.07097 & 58701.22 & 192.07058 & 0.39 & $2.05^{12} \mathrm{C}_{12}{ }^{1} \mathrm{H}_{13}{ }^{35} \mathrm{Cl}_{1}$ & 6.0 \\
\hline 194.06816 & 18904.11 & 194.06763 & 0.53 & $2.73^{12} \mathrm{C}_{12}{ }^{1} \mathrm{H}_{13}{ }^{37} \mathrm{Cl}_{1}$ & 6.0 \\
\hline
\end{tabular}


Data:1912052

Comment:SP-207 GC/EI

Description:

Ionization Mode:El+

History:Average(MS[1] 14.10..14.14)

Charge number: 1

Element: ${ }^{12} \mathrm{C}: 0$.. 50, ${ }^{1} \mathrm{H}: 0$.. 100, ${ }^{14} \mathrm{~N}: 0$.. $3,{ }^{16} \mathrm{O}: 0$.. 6

Tolerance:2.00[mDa]
Acquired:12:00:00 AM

Operator:AccuTOF

m/z Calibration File:Calib1912002

Created:12/20/2019 11:33:28 AM

Created by:AccuTOF

Unsaturation Number:-2.0 .. 15.0 (Fraction:Both)

Relative Intensity

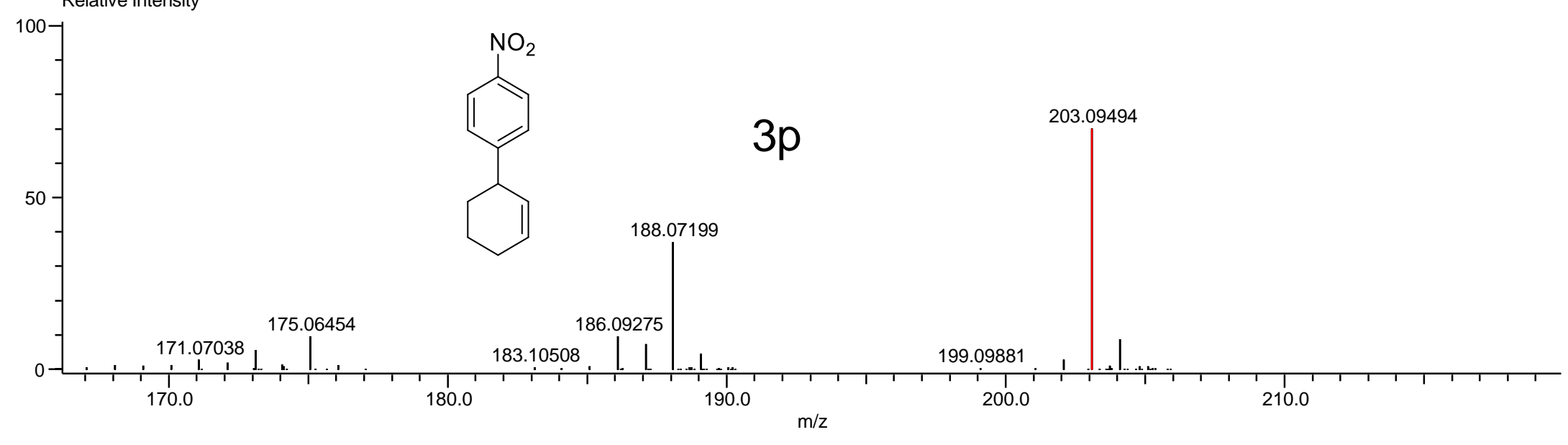

\begin{tabular}{|c|r|r|r|r|r|r|}
\hline Mass & Intensity & Calc. Mass & $\begin{array}{c}\text { Mass Difference } \\
{[\mathrm{mDa}]}\end{array}$ & $\begin{array}{c}\text { Mass Difference } \\
{[\mathrm{ppm}]}\end{array}$ & Possible Formula & Unsaturation Number \\
\hline 203.09494 & 250361.45 & 203.09463 & 0.31 & $1.54{ }^{12} \mathrm{C}_{12} \mathrm{H}_{13^{14}} \mathrm{~N}_{1}{ }^{16} \mathrm{O}_{2}$ & 7.0 \\
\hline
\end{tabular}


Data:2001064B

Comment:BG0059 GC/EI

Description:

Ionization Mode:EI+

History:Average(MS[1] 14.86..14.88)

Charge number: 1

Element: ${ }^{12} \mathrm{C}: 0$.. 50, ${ }^{1} \mathrm{H}: 0$.. 100, ${ }^{16} \mathrm{O}: 0$.. 4, ${ }^{32 \mathrm{~S}: 0} . .1$

Tolerance:3.00[mDa]

\section{Relative Intensity}

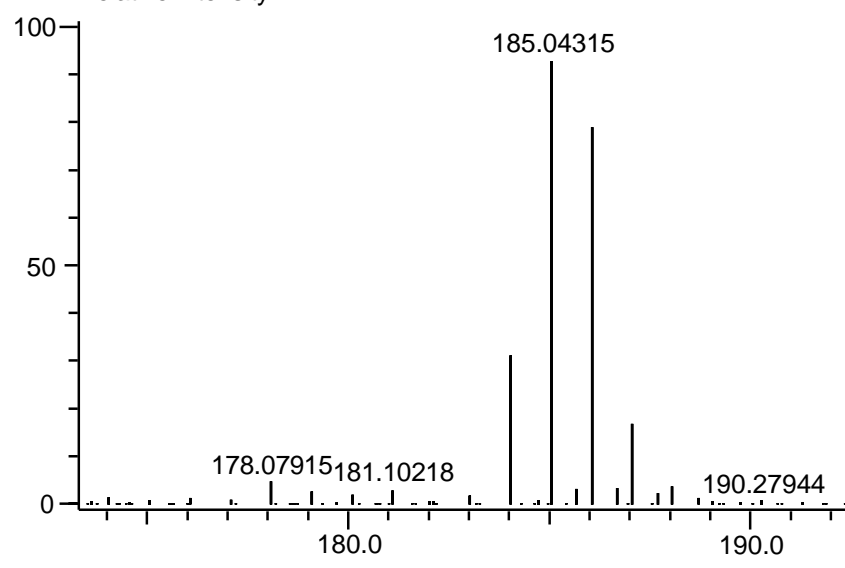

$3 q$

$3 q$

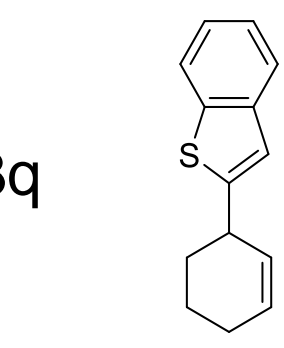

Acquired:1/30/2020 12:09:12 PM

Operator:AccuTOF

m/z Calibration File:CalibEI220120

Created:1/30/2020 2:46:54 PM

Created by:AccuTOF

Unsaturation Number:-2.0 .. 20.0 (Fraction:Both)

214.08127

\begin{tabular}{|l|r|r|r|r|r|r|}
\hline Mass & Intensity & Calc. Mass & $\begin{array}{c}\text { Mass Difference } \\
{[\mathrm{mDa}]}\end{array}$ & $\begin{array}{c}\text { Mass Difference } \\
{[\mathrm{ppm}]}\end{array}$ & Possible Formula & Unsaturation Number \\
\hline 214.08127 & 1419753.45 & 214.08162 & -0.35 & -1.62 & ${ }^{12} \mathrm{C}_{14}{ }^{1} \mathrm{H}_{14}{ }^{32} \mathrm{~S}_{1}$ & 9.0 \\
\hline
\end{tabular}


Data: 1912050

Comment:SP-204 GC/EI

Description:

Ionization Mode:EI+

History:Average(MS[1] 13.12..13.18)

Charge number: 1

Element: ${ }^{12} \mathrm{C}: 0$.. 50, ${ }^{1} \mathrm{H}: 0$.. 100, ${ }^{16} \mathrm{O}: 0$.. 6

Relative Intensity

$$
100-
$$
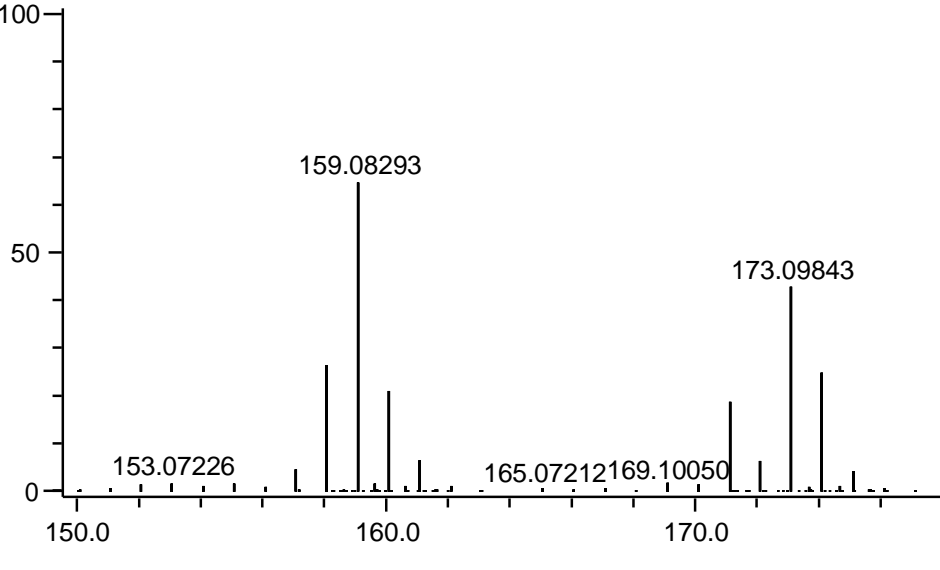

$12 \mathrm{a}$

Tolerance:5.00[mDa]

cquired:12:00:00 AM

Operator:AccuTOF

m/z Calibration File:Calib1912002

Created:12/20/2019 10:32:38 AM

Created by:AccuTOF

Unsaturation Number:-2.0 .. 15.0 (Fraction:Both)

\begin{tabular}{|l|r|r|r|r|r|r|} 
Mass & Intensity & Calc. Mass & $\begin{array}{c}\text { Mass Difference } \\
{[\mathrm{mDa}]}\end{array}$ & $\begin{array}{c}\text { Mass Difference } \\
{[\mathrm{ppm}]}\end{array}$ & Possible Formula & Unsaturation Number \\
\hline 202.13657 & 314554.88 & 202.13576 & 0.80 & $3.96^{12} \mathrm{C}_{14}{ }^{1} \mathrm{H}_{18}{ }^{16} \mathrm{O}_{1}$ & 6.0 \\
\hline
\end{tabular}


Data:1912053

Comment:SP-211 GC/EI

Description:

Ionization Mode:El+

History:Average(MS[1] 11.54..11.59)

Charge number: 1

Element: ${ }^{12} \mathrm{C}: 0$.. 50, ${ }^{1} \mathrm{H}: 0 . .100,{ }^{14} \mathrm{~N}: 0$. . 3, ${ }^{16} \mathrm{O}: 0 . .6$

Tolerance:2.00[mDa]

quired:12:00:00 AM

Operator:AccuTOF

m/z Calibration File:Calib1912002

Created:12/20/2019 12:00:05 PM

Created by:AccuTOF

Unsaturation Number:-2.0 .. 15.0 (Fraction:Both)

Relative Intensity

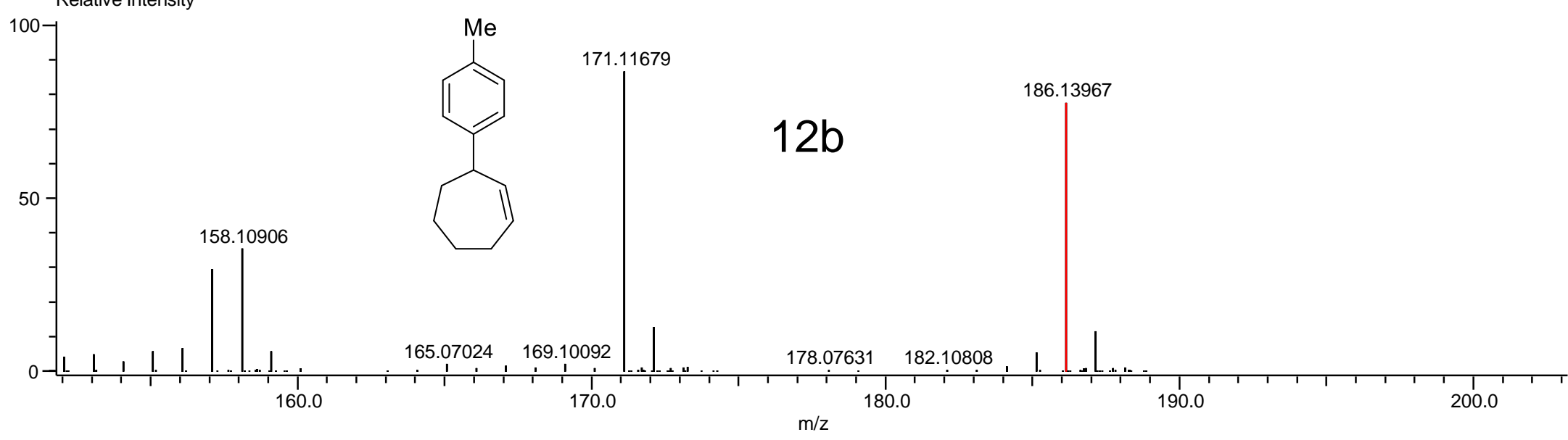

\begin{tabular}{|c|c|r|r|r|r|r|}
\hline Mass & Intensity & Calc. Mass & $\begin{array}{c}\text { Mass Difference } \\
{[\mathrm{mDa}]}\end{array}$ & $\begin{array}{c}\text { Mass Difference } \\
{[\mathrm{ppm}]}\end{array}$ & Possible Formula & Unsaturation Number \\
\hline 186.13967 & 105424.08 & 186.14085 & -1.18 & $-6.32^{12} \mathrm{C}_{14}{ }^{1} \mathrm{H}_{18}$ & 6.0 \\
\hline
\end{tabular}


Data:2002017

Comment:SP-242 GC/El

Description:

Ionization Mode:El+

History:Average(MS[1] 13.97..13.99)-1*Average(MS[1] 13.89..13.90),\#0-

Charge number: 1

Element: ${ }^{12} \mathrm{C}: 0$.. 50, ${ }^{1} \mathrm{H}: 0$.. 100, ${ }^{14} \mathrm{~N}: 0$.. 1, ${ }^{16} \mathrm{O}: 0$..

Tolerance:5.00[mDa]

Acquired:2/7/2020 9:58:14 AM

Operator:AccuTOF

$\mathrm{m} / \mathrm{z}$ Calibration File:CalibEI220120

Created:2/7/2020 10:25:08 AM

Created by:AccuTOF

Unsaturation Number:-2.0 .. 9.0 (Fraction:Both)

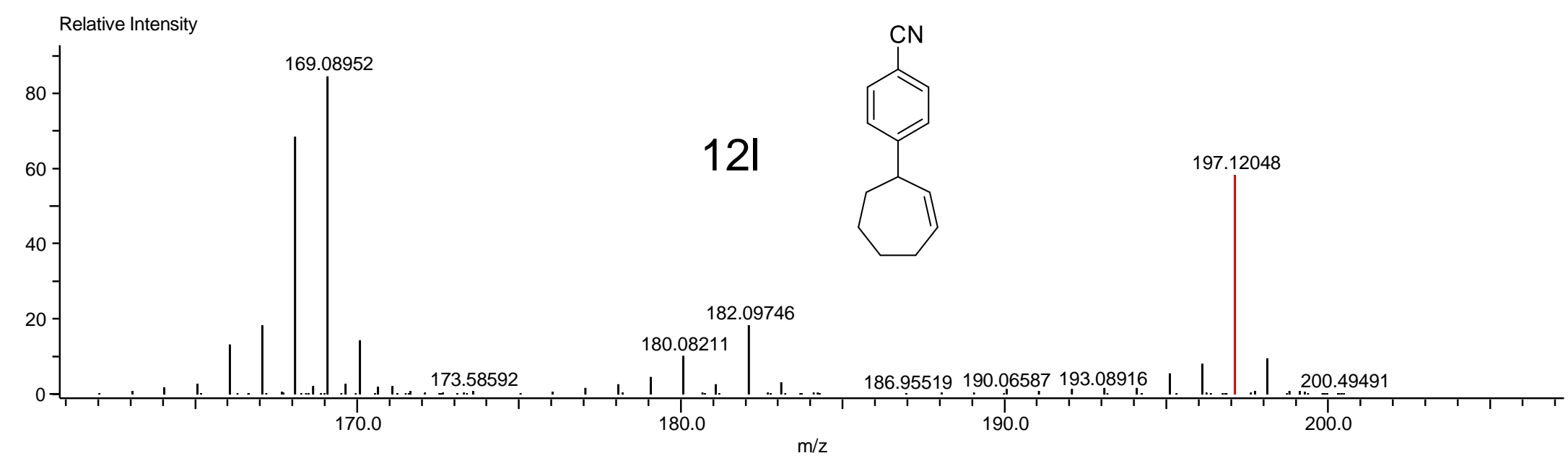

\begin{tabular}{|c|c|r|r|r|r|r|}
\hline Mass & Intensity & Calc. Mass & $\begin{array}{c}\text { Mass Difference } \\
{[\mathrm{mDa}]}\end{array}$ & $\begin{array}{c}\text { Mass Difference } \\
{[\mathrm{ppm}]}\end{array}$ & Possible Formula & Unsaturation Number \\
\hline 197.12048 & 223898.97 & 197.12045 & 0.03 & $0.14{ }^{12} \mathrm{C}_{14}{ }^{1} \mathrm{H}_{15}{ }^{14} \mathrm{~N}_{1}$ & 8.0 \\
\hline
\end{tabular}


Data:2001063

Comment:BG0053 GC/E

Description:

Ionization Mode:EI+

History:Average(MS[1] 14.25..14.26)

Charge number: 1

Element: ${ }^{12} \mathrm{C}: 0$.. 50, ${ }^{1} \mathrm{H}: 0$.. 100, ${ }^{16} \mathrm{O}: 0$.. 4

Tolerance:3.00[mDa]

Acired:12:00:00 AM

Operator:AccuTOF

m/z Calibration File:CalibEI220120

Created:1/30/2020 10:39:02 AM

Created by:AccuTOF

Unsaturation Number:-2.0 .. 20.0 (Fraction:Both)
Relative Intensity

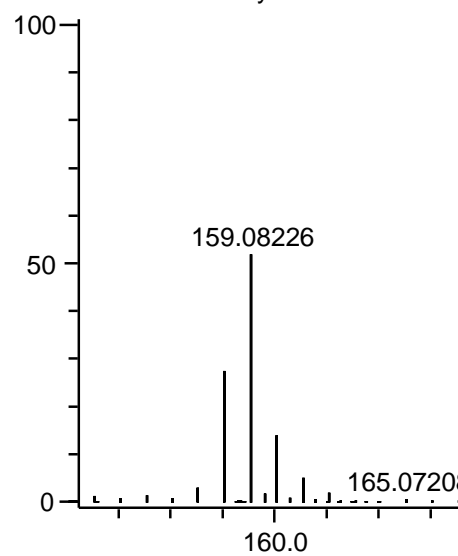

173.09781
$13 a$

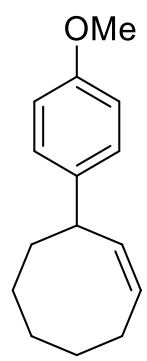

$201.12884 \quad 207.03491$

\author{
216.15157
}

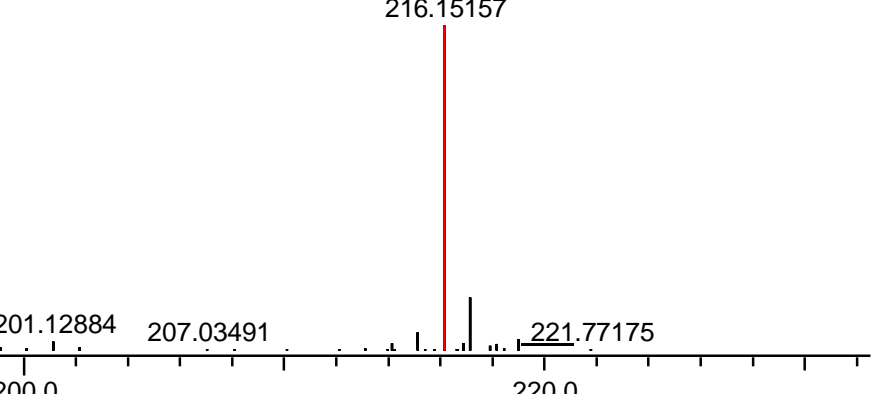

\begin{tabular}{|l|r|r|r|r|r|r|}
\hline Mass & Intensity & Calc. Mass & $\begin{array}{c}\text { Mass Difference } \\
{[\mathrm{mDa}]}\end{array}$ & $\begin{array}{c}\text { Mass Difference } \\
{[\mathrm{ppm}]}\end{array}$ & Possible Formula & Unsaturation Number \\
\hline 216.15157 & 1106558.01 & 216.15141 & 0.16 & 0.72 & ${ }^{12} \mathrm{C}_{15}{ }^{1} \mathrm{H}_{20}{ }^{16} \mathrm{O}_{1}$ & 6.0 \\
\hline
\end{tabular}


Data:1912054

Comment:SP-213 GC/EI

Description:

Ionization Mode:EI+

History:Average(MS[1] 18.61..18.66)

Charge number: 1

Element: ${ }^{12} \mathrm{C}: 0$.. 50, ${ }^{1 \mathrm{H}: 0}$.. 100, ${ }^{14} \mathrm{~N}: 0$. . 3, ${ }^{16} \mathrm{O}: 0$.. 6

Tolerance:2.00[mDa]$$
100-
$$

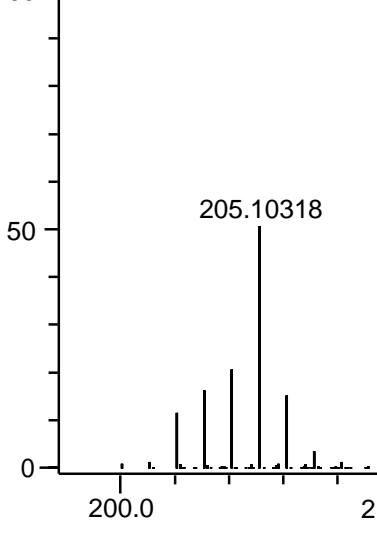

$13 c$

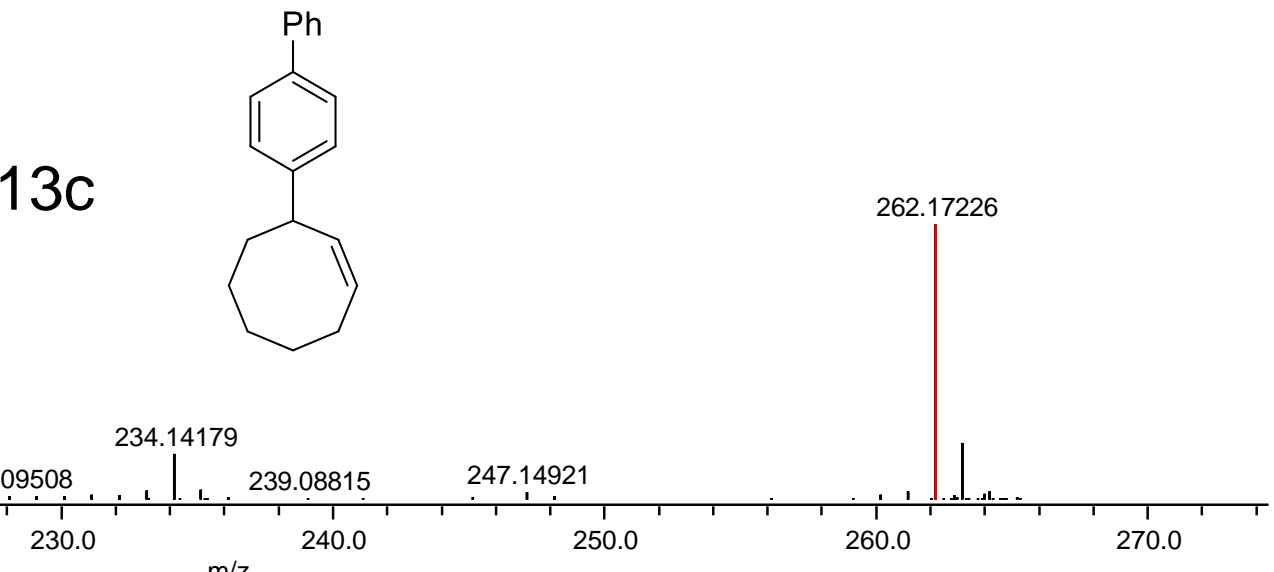

\begin{tabular}{|c|c|r|r|r|r|r|}
\hline Mass & Intensity & Calc. Mass & $\begin{array}{c}\text { Mass Difference } \\
{[\mathrm{mDa}]}\end{array}$ & $\begin{array}{c}\text { Mass Difference } \\
{[\mathrm{ppm}]}\end{array}$ & Possible Formula & Unsaturation Number \\
\hline 262.17226 & 260671.04 & 262.17215 & 0.11 & 0.41 & ${ }^{12} \mathrm{C}_{20}{ }^{1} \mathrm{H}_{22}$ & 10.0 \\
\hline
\end{tabular}

Acquired:12/20/2019 12:06:42 PM

Operator:AccuTOF

m/z Calibration File:Calib1912002

Created:12/20/2019 1:56:34 PM

Created by:AccuTOF

Unsaturation Number:-2.0 .. 15.0 (Fraction:Both) 
Data:2002027

\section{Comment:BG0075 GC/EI}

Description:

Ionization Mode:EI+

History:Average(MS[1] 11.74..11.79)-1*Average(MS[1] 11.55...11.60),\#0-

Charge number:1

Element: ${ }^{12} \mathrm{C}: 0$.. 50, ${ }^{1} \mathrm{H}: 0$.. 100, ${ }^{19} \mathrm{~F}: 0$.. 1, ${ }^{16} \mathrm{O}: 0$.. 3

Tolerance: $5.00[\mathrm{mDa}]$

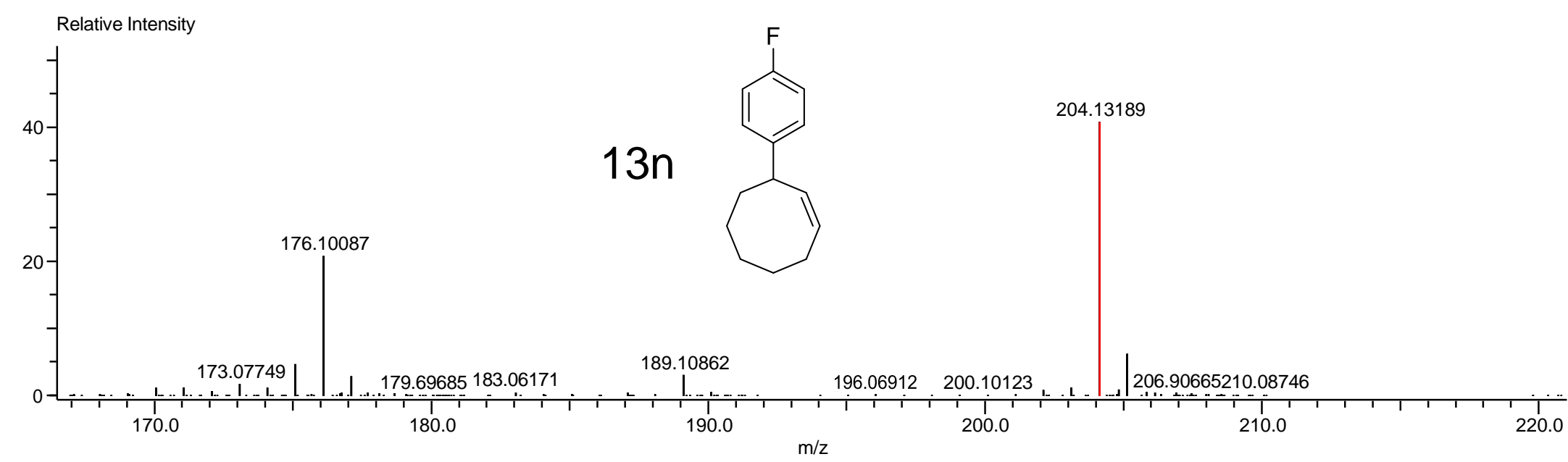

Acquired:12:00:00 AM

Operator:AccuTOF

$\mathrm{m} / \mathrm{z}$ Calibration File:CalibEI220120

Created:2/14/2020 2:56:18 PM

Created by:AccuTOF

Unsaturation Number:-2.0 .. 15.0 (Fraction:Both)

\begin{tabular}{|l|l|r|r|r|r|r|}
\hline Mass & Intensity & Calc. Mass & $\begin{array}{c}\text { Mass Difference } \\
{[\mathrm{mDa}]}\end{array}$ & $\begin{array}{c}\text { Mass Difference } \\
{[\mathrm{ppm}]}\end{array}$ & Possible Formula & Unsaturation Number \\
\hline 204.13189 & 1421759.86 & 204.13143 & 0.47 & 2.28 & ${ }^{12} \mathrm{C}_{14}{ }^{1} \mathrm{H}_{17} 7^{19} \mathrm{~F}_{1}$ & 6.0 \\
\hline
\end{tabular}


Data:2002029

Comment:SP-260 GC/E

Description:

lonization Mode:EI+

History:Average(MS[1] 10.51..10.56)-1*Average(MS[1] 10.32..10.36),\#0-

Charge number: 1

Element: ${ }^{12} \mathrm{C}: 0$.. 50, ${ }^{1} \mathrm{H}: 0$.. 100, ${ }^{16} \mathrm{O}: 0$.. 3

Tolerance:5.00[mDa]

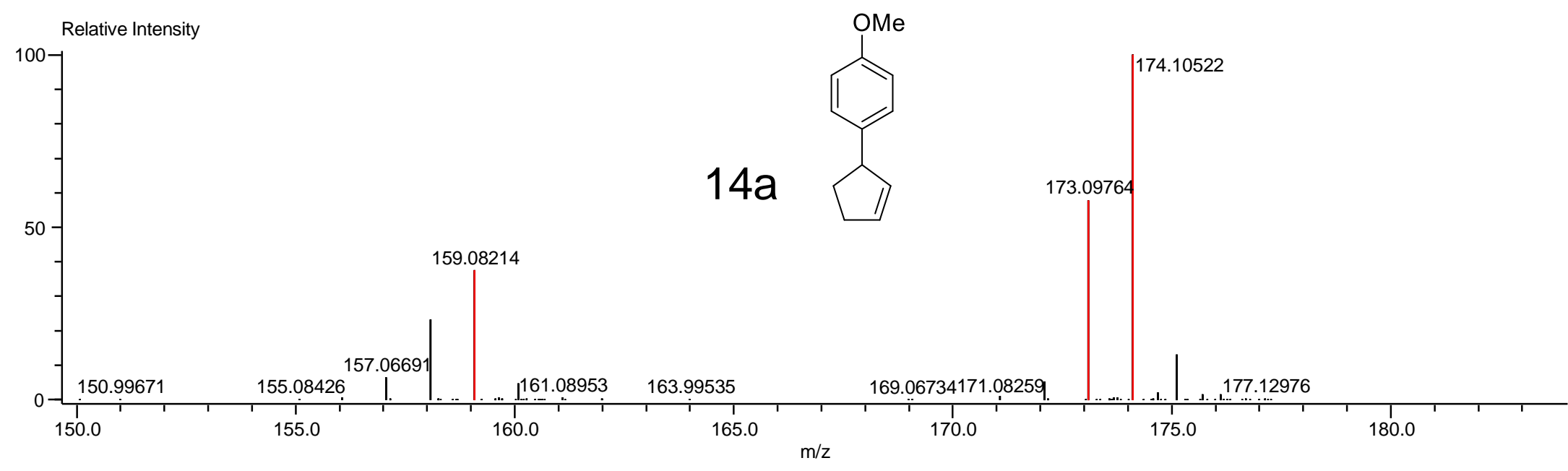

\begin{tabular}{|c|c|c|c|c|c|c|}
\hline Mass & Intensity & Calc. Mass & $\begin{array}{l}\text { Mass Difference } \\
{[\mathrm{mDa}]}\end{array}$ & $\begin{array}{l}\text { Mass Difference } \\
{[\mathrm{ppm}]}\end{array}$ & Possible Formula & Unsaturation Number \\
\hline 159.08214 & 78221.05 & 159.08099 & 1.15 & 7.26 & ${ }^{12} \mathrm{C}_{11}{ }^{1} \mathrm{H}_{11}{ }^{16} \mathrm{O}_{1}$ & 6.5 \\
\hline 173.09764 & 120610.84 & 173.09664 & 1.00 & 5.76 & ${ }^{12} \mathrm{C}_{12}{ }^{1} \mathrm{H}_{13}{ }^{16} \mathrm{O}_{1}$ & 6.5 \\
\hline 174.10522 & 208758.17 & 174.10446 & 0.76 & 4.35 & ${ }^{12} \mathrm{C}_{12}{ }^{1} \mathrm{H}_{14}{ }^{16} \mathrm{O}_{1}$ & 6.0 \\
\hline
\end{tabular}

Acquired:12:00:00 AM

Operator:AccuTOF

m/z Calibration File:CalibEI220120

Created:2/14/2020 3:48:26 PM

Created by:AccuTOF

Unsaturation Number:-2.0 .. 15.0 (Fraction:Both) 
Data:2001065

Comment:BG0061 GC/E

Description:

Ionization Mode:EI+

History:Average(MS[1] 15.33..15.37)

Charge number: 1

Element: ${ }^{12} \mathrm{C}: 0$.. 50, ${ }^{1} \mathrm{H}: 0$.. 100, ${ }^{32} \mathrm{~S}: 0$.. 1

Relative Intensity

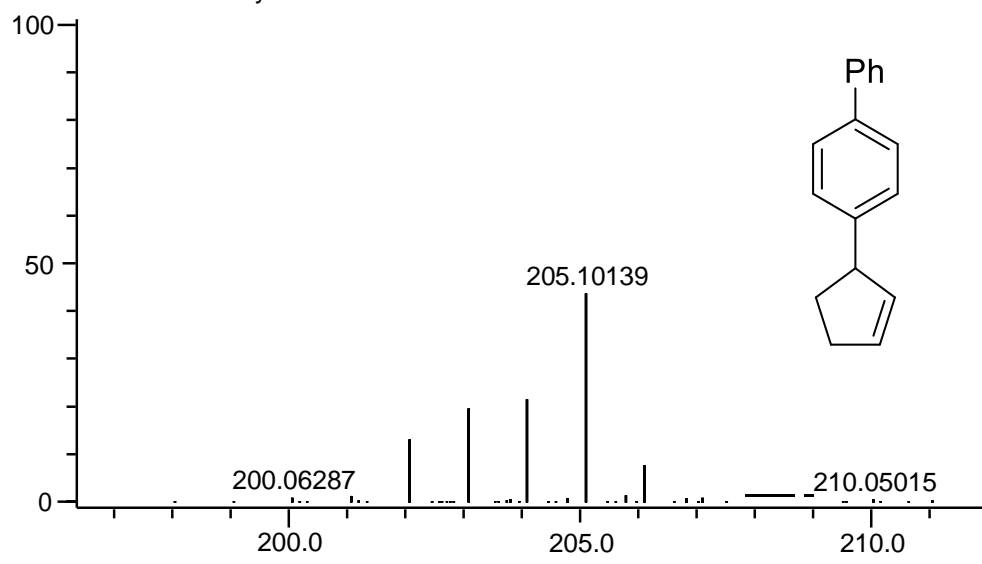

Tolerance:3.00[mDa]
Acquired:12:00:00 AM

Operator:AccuTOF

$\mathrm{m} / \mathrm{z}$ Calibration File:CalibEI220120

Created:1/30/2020 11:36:46 AM

Created by:AccuTOF

Unsaturation Number:-2.0 .. 20.0 (Fraction:Both)

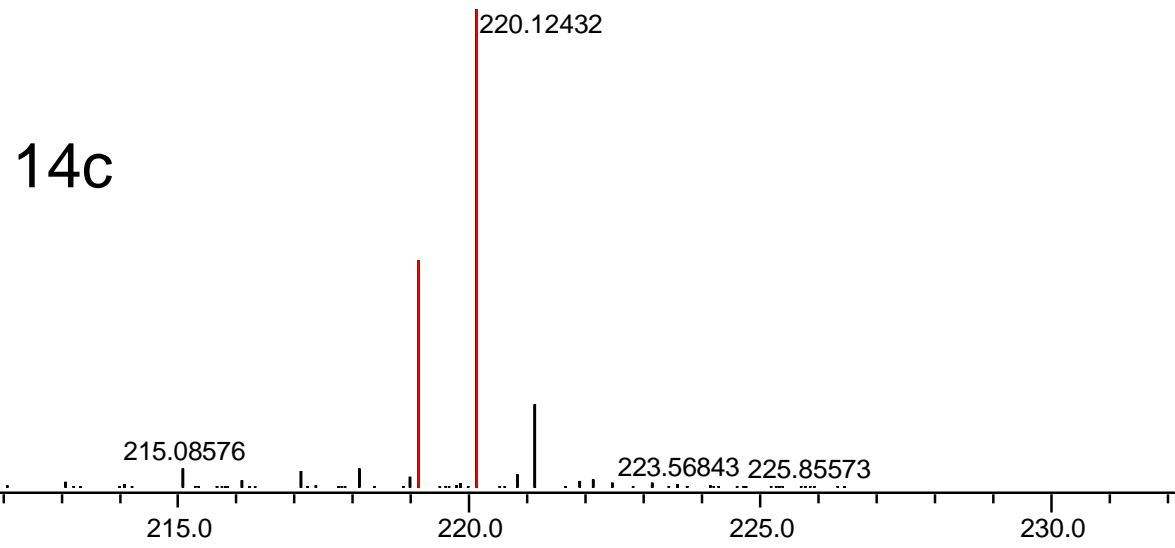

\begin{tabular}{|c|r|r|r|r|r|r|}
\hline Mass & Intensity & Calc. Mass & $\begin{array}{c}\text { Mass Difference } \\
{[\mathrm{mDa}]}\end{array}$ & $\begin{array}{c}\text { Mass Difference } \\
{[\mathrm{ppm}]}\end{array}$ & Possible Formula & Unsaturation Number \\
\hline 219.11671 & 814515.39 & 219.11738 & -0.67 & -3.04 & ${ }^{12} \mathrm{C}_{17}{ }^{1} \mathrm{H}_{15}$ & 10.5 \\
\hline 220.12432 & 1719241.47 & 220.12520 & -0.88 & -4.01 & ${ }^{12} \mathrm{C}_{17}{ }^{1} \mathrm{H}_{16}$ & 10.0 \\
\hline
\end{tabular}


Data:2001066B

Comment:BG0066 GC/E

Description:

Ionization Mode:El+

History:Average(MS[1] 12.89..12.90)-1*Average(MS[1] 12.81..12.82),\#0-

Charge number: 1

Element: ${ }^{12} \mathrm{C}: 0$.. 50, ${ }^{1} \mathrm{H}: 0$.. 100, ${ }^{16} \mathrm{O}: 0$.. 4

Tolerance:3.00[mDa]

$$
100-
$$
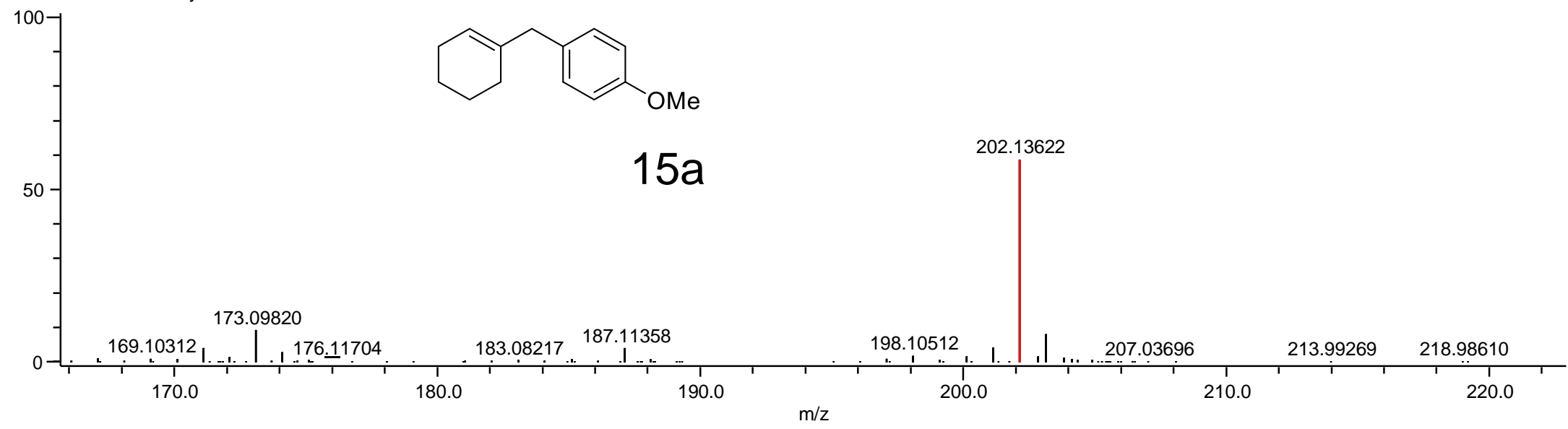

\begin{tabular}{|c|c|r|r|r|r|r|}
\hline Mass & Intensity & Calc. Mass & $\begin{array}{c}\text { Mass Difference } \\
{[\mathrm{mDa}]}\end{array}$ & $\begin{array}{c}\text { Mass Difference } \\
{[\mathrm{ppm}]}\end{array}$ & Possible Formula & Unsaturation Number \\
\hline 202.13622 & 1116032.68 & 202.13576 & 0.46 & 2.28 & ${ }^{12} \mathrm{C}_{14}{ }^{1} \mathrm{H}_{18}{ }^{16} \mathrm{O}_{1}$ & 6.0 \\
\hline
\end{tabular}

Acquired:1/30/2020 12:36:03 PM

Operator:AccuTOF

$\mathrm{m} / \mathrm{z}$ Calibration File:CalibEI220120

Created:1/30/2020 2:39:09 PM

Created by:AccuTOF

Unsaturation Number:-2.0 .. 20.0 (Fraction:Both) 
Data:2002030

Comment:SP-262 GC/EI

Description:

lonization Mode:El+

History:Average(MS[1] 13.74..13.79)-1*Average(MS[1] 13.39..13.46),\#0-

Charge number:1

Element: ${ }^{12} \mathrm{C}: 0$.. 50, ${ }^{1} \mathrm{H}: 0$.. 100, ${ }^{14} \mathrm{~N}: 0$.. 1
Acquired:2/14/2020 3:58:29 PM

Operator:AccuTOF

$\mathrm{m} / \mathrm{z}$ Calibration File:CalibEI220120

Created:2/14/2020 4:25:24 PM

Created by:AccuTOF

Unsaturation Number:-2.0 .. 15.0 (Fracti...

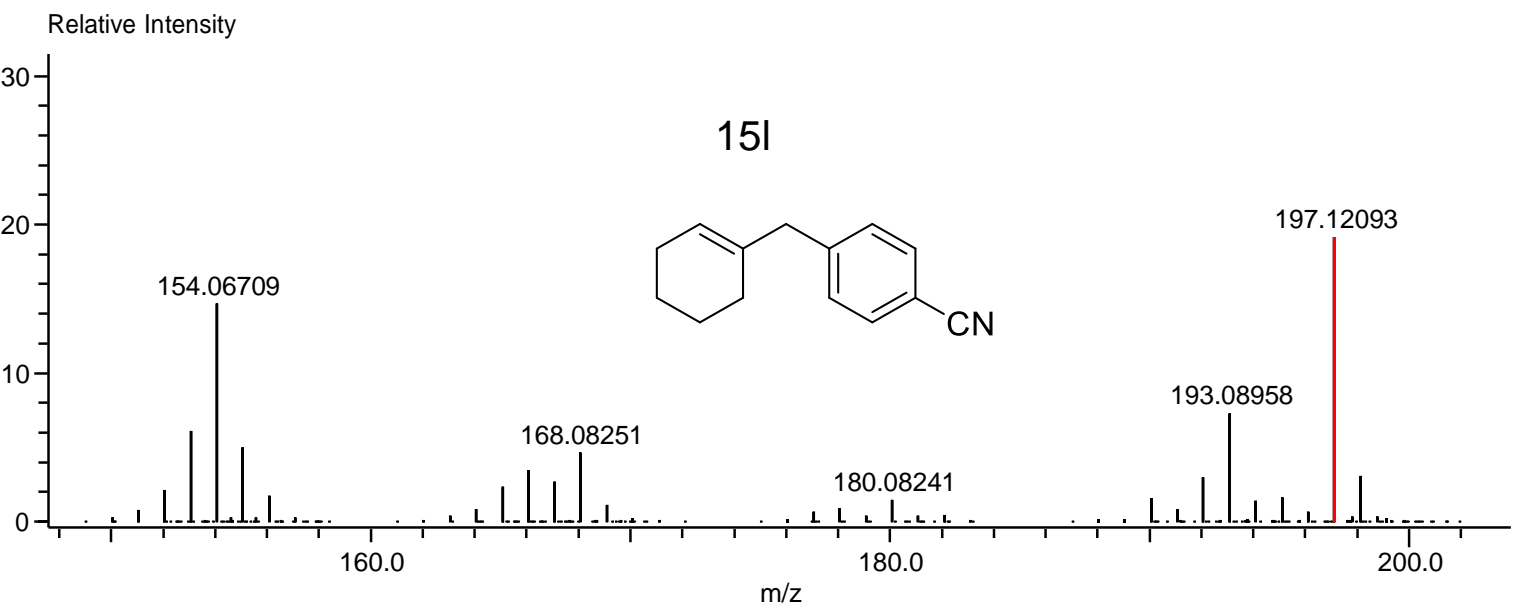

\begin{tabular}{|c|r|r|r|r|r|r|}
\hline Mass & Intensity & Calc. Mass & $\begin{array}{c}\text { Mass Difference } \\
{[\mathrm{mDa}]}\end{array}$ & $\begin{array}{c}\text { Mass Difference } \\
{[\mathrm{ppm}]}\end{array}$ & Possible Formula & Unsaturation Number \\
\hline 197.12093 & 477749.77 & 197.12045 & 0.48 & $2.43{ }^{12} \mathrm{C}_{14}{ }^{1} \mathrm{H}_{15}{ }^{14} \mathrm{~N}_{1}$ & 8.0 \\
\hline
\end{tabular}


Data:1912055

Comment:SP-215 GC/E

Description:

lonization Mode:EI+

History:Average(MS[1] 15.68..15.73)-1*Average(MS[1] 15.36..15.41),\#0-

Charge number: 1

Element: ${ }^{12} \mathrm{C}: 0$.. 50, ${ }^{1} \mathrm{H}: 0$.. 100, ${ }^{16} \mathrm{O}: 0$.. 6

Tolerance:2.00[mDa]

Relative Intensity

$100-$

7

180.0
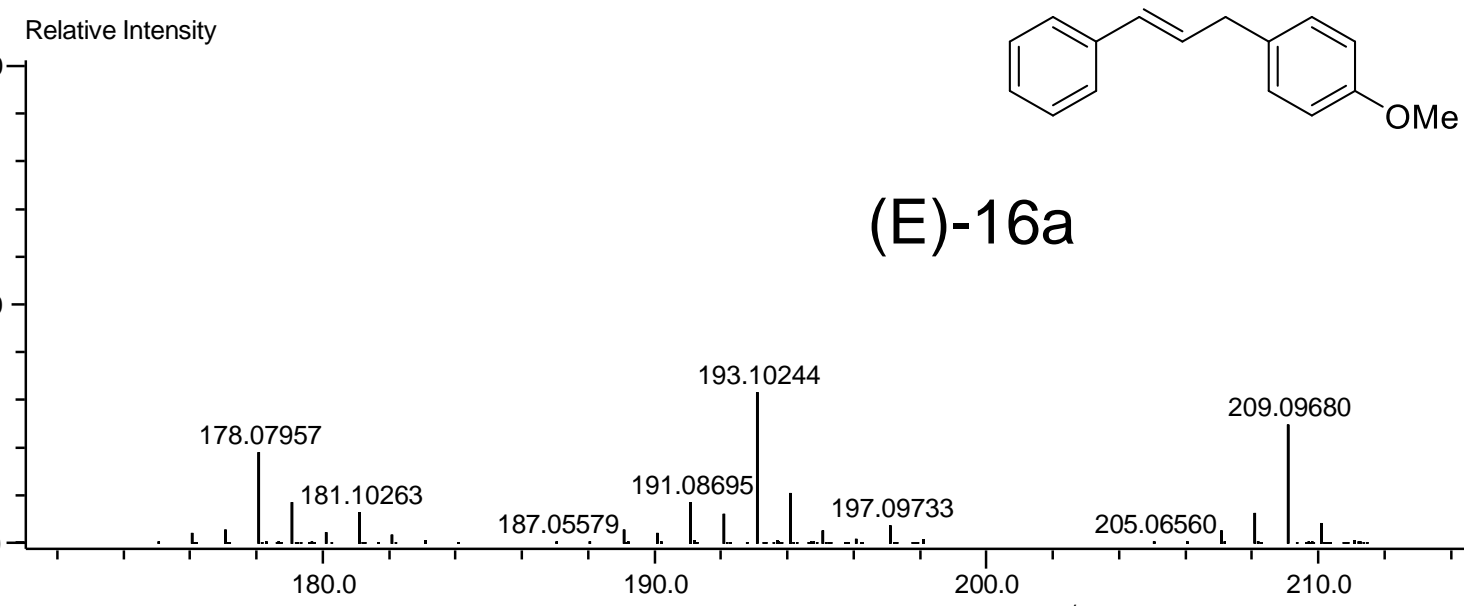

OMe

(E)-16a

\begin{tabular}{|c|c|r|r|r|r|r|}
\hline Mass & Intensity & Calc. Mass & $\begin{array}{c}\text { Mass Difference } \\
{[\mathrm{mDa}]}\end{array}$ & $\begin{array}{c}\text { Mass Difference } \\
{[\mathrm{ppm}]}\end{array}$ & Possible Formula & Unsaturation Number \\
\hline 224.11975 & 316108.09 & 224.12011 & -0.37 & -1.64 & ${ }^{12} \mathrm{C}_{16}{ }^{1} \mathrm{H}_{16}{ }^{16} \mathrm{O}_{1}$ & 9.0 \\
\hline
\end{tabular}

Acquired:12/20/2019 12:33:30 PM

Operator:AccuTOF

m/z Calibration File:Calib1912002

Created:12/20/2019 1:59:44 PM

Created by:AccuTOF

Unsaturation Number:-2.0 .. 15.0 (Fraction:Both) 
Data:2002020

Comment:SP-256 GC/E

Description:

Ionization Mode:El+

History:Average(MS[1] 16.46..16.50)-1*Average(MS[1] 16.27..16.32),\#0-

Charge number: 1

Tolerance:5.00[mDa]

Element: ${ }^{12} \mathrm{C}: 0$.. 50, ${ }^{1} \mathrm{H}: 0$.. 100, ${ }^{14} \mathrm{~N}: 0$.. 1

Relative Intensity
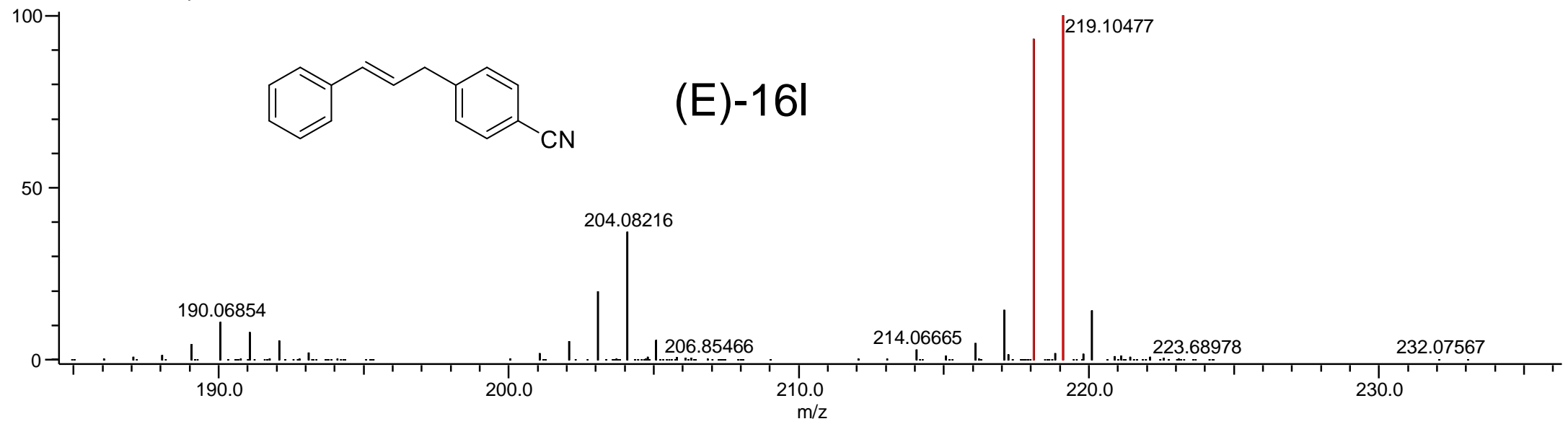

\begin{tabular}{|l|r|r|r|r|r|r|}
\hline Mass & Intensity & Calc. Mass & $\begin{array}{c}\text { Mass Difference } \\
{[\mathrm{mDa}]}\end{array}$ & $\begin{array}{c}\text { Mass Difference } \\
{[\mathrm{ppm}]}\end{array}$ & Possible Formula & Unsaturation Number \\
\hline 218.09752 & 909481.27 & 218.09697 & 0.55 & 2.51 & ${ }^{12} \mathrm{C}_{16}{ }^{1} \mathrm{H}_{12}{ }^{14} \mathrm{~N}_{1}$ & 11.5 \\
\hline 219.10477 & 975157.24 & 219.10480 & -0.02 & -0.11 & ${ }^{12} \mathrm{C}_{16}{ }^{1} \mathrm{H}_{13}{ }^{14} \mathrm{~N}_{1}$ & 11.0 \\
\hline
\end{tabular}


Data:2002019

Comment:SP-253 GC/E

Description:

Ionization Mode:El+

History:Average(MS[1] 15.56..15.58)-1*Average(MS[1] 15.52..15.53),\#0-

Charge number: 1

Element: ${ }^{12} \mathrm{C}: 0 . .50,{ }^{1} \mathrm{H}: 0$.. 100, ${ }^{19} \mathrm{~F}: 0$.. 1, ${ }^{14} \mathrm{~N}: 0$.. 1, ${ }^{16} \mathrm{O}: 0$..
Acquired:12:00:00 AM

Operator:AccuTOF

$\mathrm{m} / \mathrm{z}$ Calibration File:CalibEI220120

Created:2/7/2020 11:16:51 AM

Created by:AccuTOF

Unsaturation Number:-2.0 .. 9.0 (Fraction:Both)

Relative Intensity

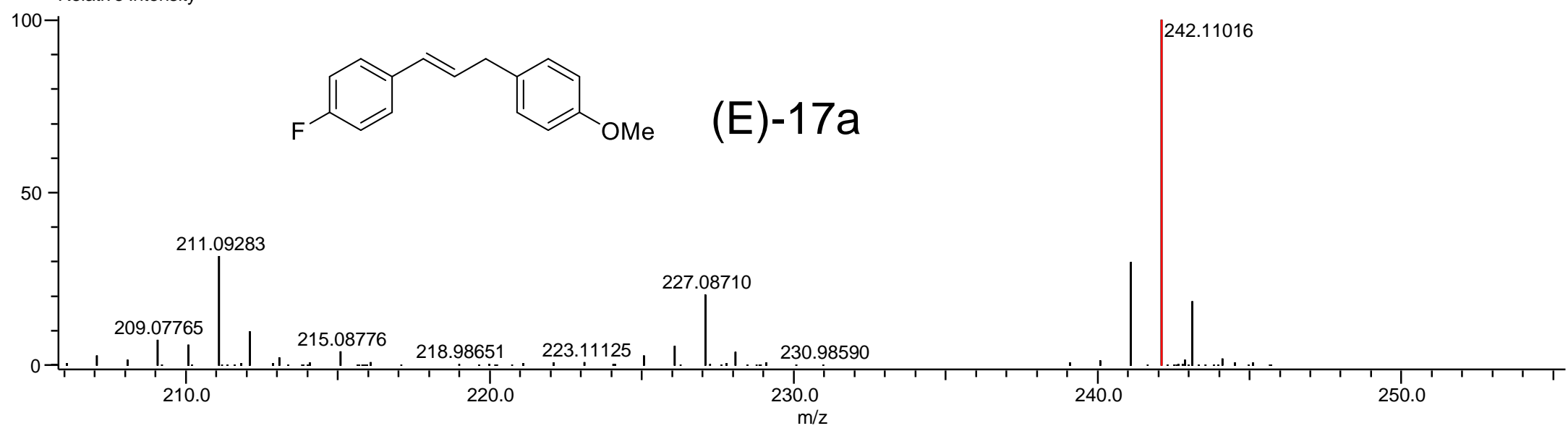

\begin{tabular}{|l|r|r|r|r|r|r|}
\hline Mass & Intensity & Calc. Mass & $\begin{array}{c}\text { Mass Difference } \\
{[\mathrm{mDa}]}\end{array}$ & $\begin{array}{c}\text { Mass Difference } \\
{[\mathrm{ppm}]}\end{array}$ & Possible Formula & Unsaturation Number \\
\hline 242.11016 & 381865.73 & 242.11069 & -0.53 & -2.19 & ${ }^{12} \mathrm{C}_{16}{ }^{1} \mathrm{H}_{15}{ }^{19} \mathrm{~F}_{1}{ }^{16} \mathrm{O}_{1}$ & 9.0 \\
\hline
\end{tabular}


Data:2002022

Comment:SP-258 GC/EI

Description:

Ionization Mode:El+

History:Average(MS[1] 16.50..16.55)-1*Average(MS[1] 16.24..16.30),\#0-

Charge number: 1

Tolerance: $5.00[\mathrm{mDa}]$

Element: ${ }^{12} \mathrm{C}: 0$.. 50, ${ }^{1} \mathrm{H}: 0$.. 100, ${ }^{19} \mathrm{~F}: 0$.. $1,{ }^{14} \mathrm{~N}: 0$..

Relative Intensity

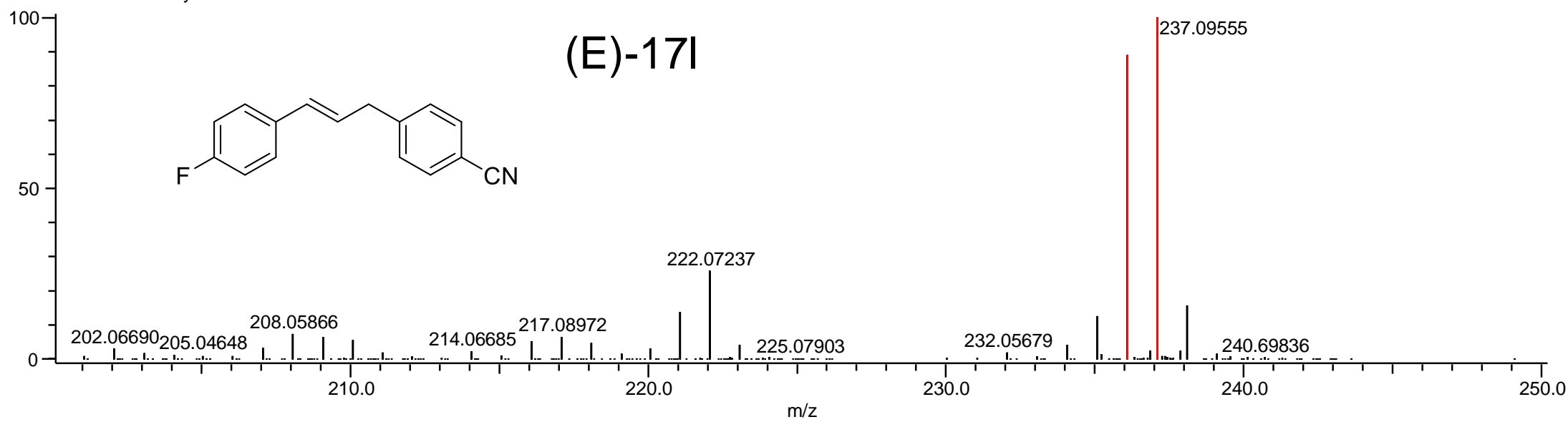

\begin{tabular}{|l|r|r|r|r|r|r|}
\hline Mass & Intensity & Calc. Mass & $\begin{array}{c}\text { Mass Difference } \\
{[\mathrm{mDa}]}\end{array}$ & $\begin{array}{c}\text { Mass Difference } \\
{[\mathrm{ppm}]}\end{array}$ & Possible Formula & Unsaturation Number \\
\hline 236.08811 & 1635290.09 & 236.08755 & 0.55 & 2.35 & ${ }^{12} \mathrm{C}_{16}{ }^{1} \mathrm{H}_{11}{ }^{19} \mathrm{~F}_{1}{ }^{14} \mathrm{~N}_{1}$ & 11.5 \\
\hline 237.09555 & 1836232.36 & 237.09538 & 0.17 & 0.74 & ${ }^{12} \mathrm{C}_{16}{ }^{1} \mathrm{H}_{12}{ }^{19} \mathrm{~F}_{1}{ }^{14} \mathrm{~N}_{1}$ & 11.0 \\
\hline
\end{tabular}

quired:2/7/2020 12:12:22 PM

Operator:AccuTOF

$\mathrm{m} / \mathrm{z}$ Calibration File:CalibEI220120

Created:2/7/2020 1:46:01 PM

Created by:AccuTOF

Unsaturation Number:-2.0 .. 15.0 (Fraction:Both) 